Characterization of strained silicon FinFETs and the integration of a piezoelectric layer

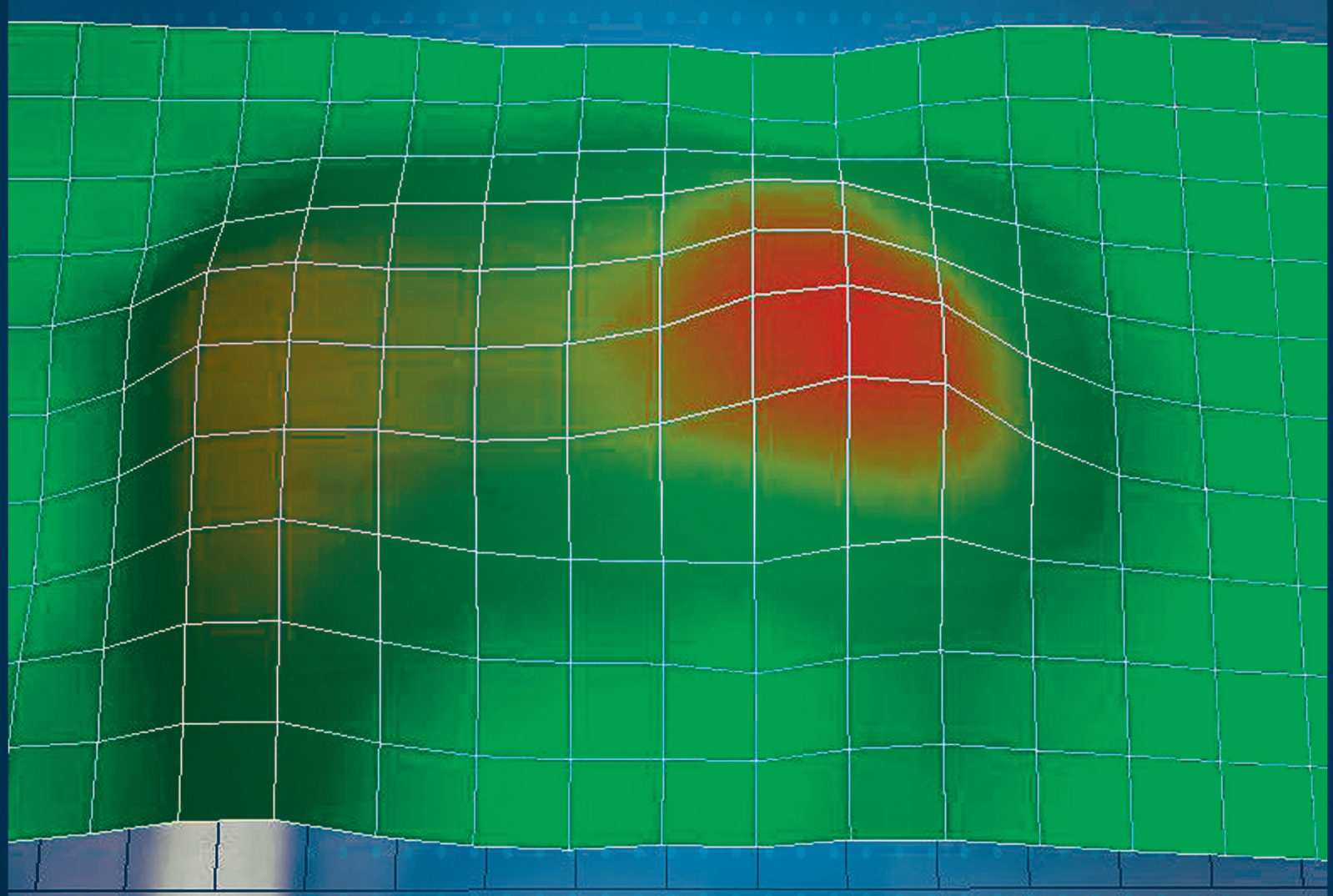




\section{CHARACTERIZATION OF STRAINED SILICON FINFETS \\ AND THE INTEGRATION OF A PIEZOELECTRIC LAYER}

Buket Kaleli 
The graduation committee consists of:

$\begin{array}{lll}\text { Chairman: } & \text { Prof.dr.ir. A.J. Mouthaan } & \text { University of Twente } \\ \text { Secretary: } & \text { Prof.dr.ir. A.J. Mouthaan } & \text { University of Twente } \\ \text { Promoter: } & \text { Prof.dr.ir. R.A.M. Wolters } & \text { University of Twente } \\ \text { Asst. promoter: } & \text { Dr. ir. R.J.E. Hueting } & \text { University of Twente } \\ \text { Referent/expert: } & \text { Dr. K. Reimann } & \text { NXP Semiconductors } \\ \text { Members: } & \text { Prof.dr. J. Schmitz } & \text { University of Twente } \\ & \text { Prof.dr.ing. A.J.H.M. Rijnders } & \text { University of Twente } \\ & \text { Prof.dr. D.J. Gravesteijn } & \text { NXP Semiconductors/ } \\ & & \text { University of Twente } \\ & \text { Prof.dr.P.M. Sarro } & \text { TU Delft }\end{array}$

This research was funded by the Dutch Technology Foundation STW, a project "Piezoelectric layer formation on a silicon channel" nr. 10176 and carried out at the Semiconductor Components group, MESA+ Institute of Nanotechnology, University of Twente, The Netherlands.

PhD thesis-University of Twente, Enschede, The Netherlands

Title: Characterization of strained silicon FinFETs and the integration of a piezoelectric layer.

Author: Buket Kaleli

ISBN: $\quad 978-90-365-0471-3$

DOI: $\quad 10.3990 / 1.9789036504713$

Cover: 2D upward response scan of a PiezoFET with $30 \mathrm{~nm}$ fin width (Chapter 5). Cover designed by Berkay Kaleli.

Copyright $@ 2013$ by Buket Kaleli

All rights reserved. No part of this publication may be reproduced, stored in a retrieval system, or transmitted, in any form or by any means, electronic, mechanical, photocopying, recording or otherwise, in whole or in part without the prior written permission of the copyright owner. 


\section{CHARACTERIZATION OF STRAINED SILICON FINFETS AND THE INTEGRATION OF A PIEZOELECTRIC LAYER}

\section{DISSERTATION}

to obtain

the degree of doctor at the University of Twente, on the authority of the rector magnificus, prof. dr. H. Brinksma, on account of the decision of the graduation committee, to be publicly defended on Wednesday the $20^{\text {th }}$ of November 2013 at 16:45

by

\section{BUKET KALELI}

born on the $24^{\text {th }}$ of September 1984

in Eskişehir, Turkey 
This dissertation is approved by:

Prof.dr.ir. Rob A.M. Wolters (promoter)

Dr.ir. Raymond J.E. Hueting (asst. promoter) 
To my beloved husband, parents and brother 


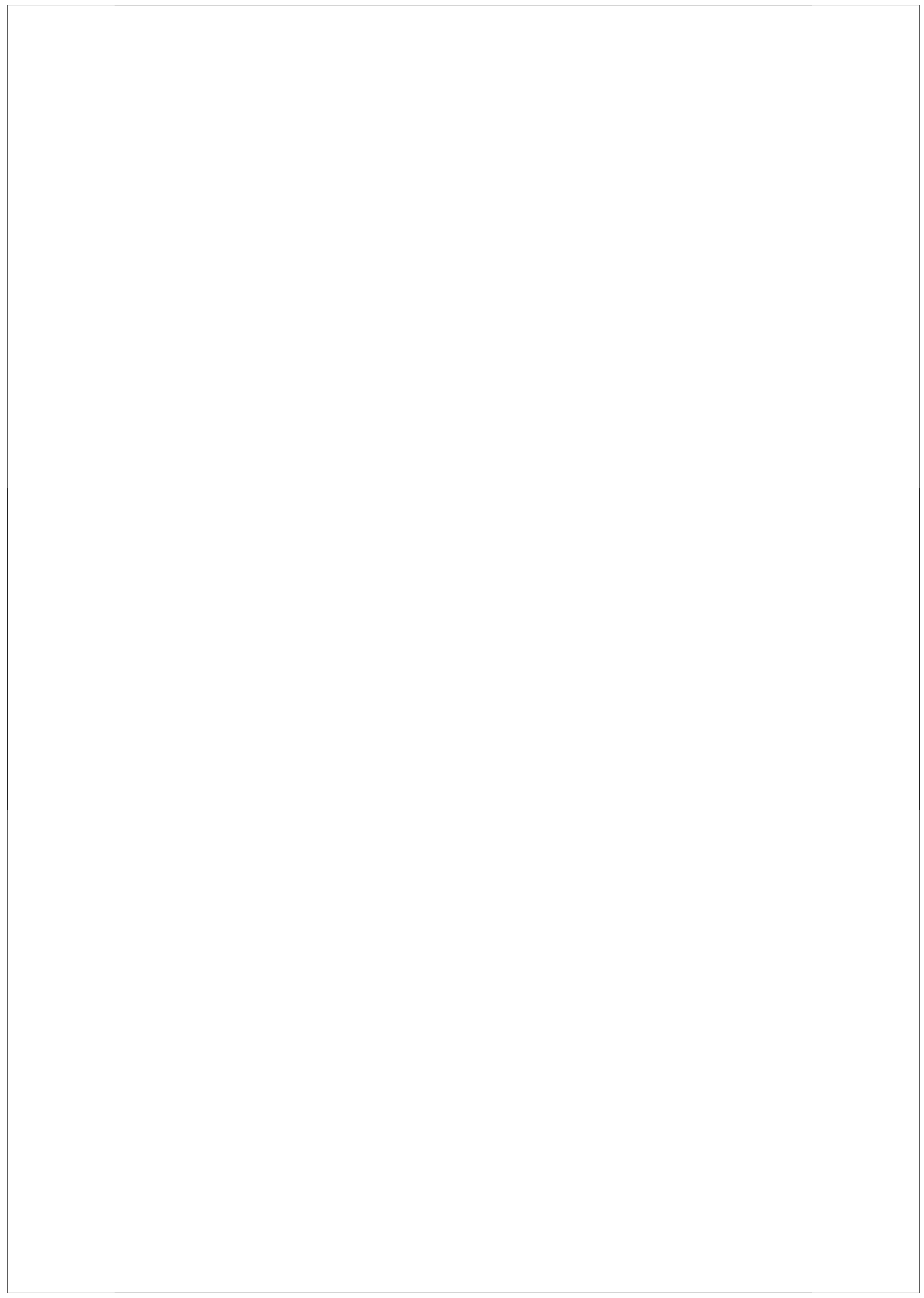




\section{Contents}

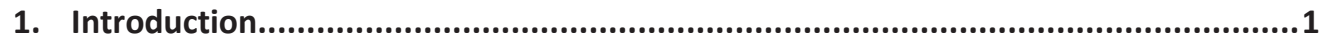

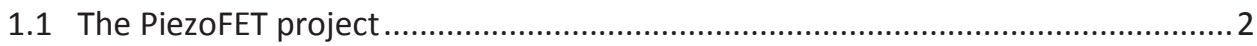

1.2 Concepts for steep sub-threshold slope FETs ...................................................

1.3 Choice of device configuration and materials................................................... 4

1.3.1 FinFETs as a replacement of MOSFETs ...................................................... 4

1.3.2 Encapsulated TiN as a gate material .........................................................

1.3.3 Converse Piezoelectric effect as a stressor ............................................... 7

1.4 Technological requirements and research questions ........................................

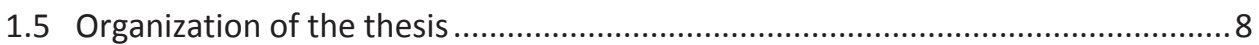

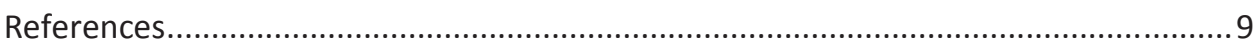

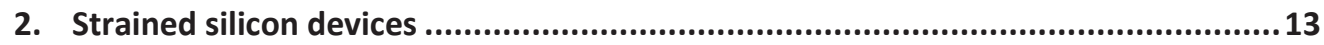

2.1 Strain formation in silicon based devices....................................................... 14

2.2 The effect of strain on the band gap and mobility........................................... 15

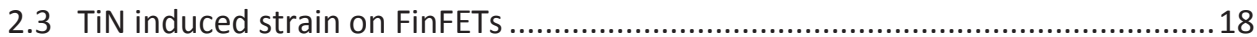

2.3.1 Characterization of strain by optical and its effects by electrical techniques

2.3.1.1 Temperature dependent $I-V$ measurements on strained devices ......21

2.3.1.2 Raman spectroscopy characterization of strain..................................23

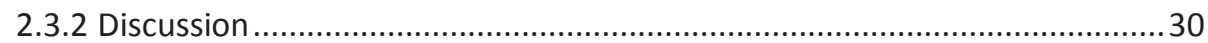

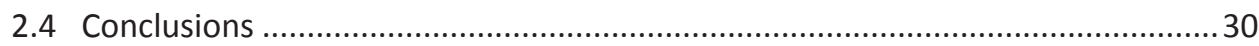

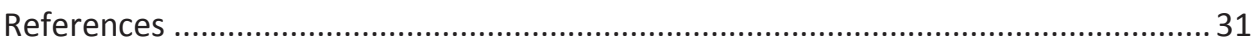

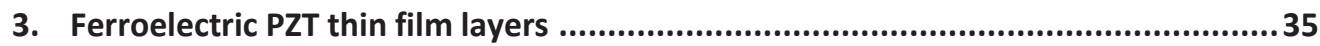

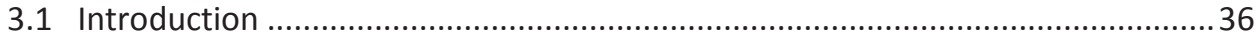

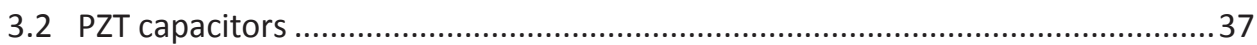

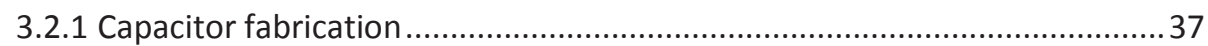


3.2.2 Characterization of the PZT stacks …..................................................... 38

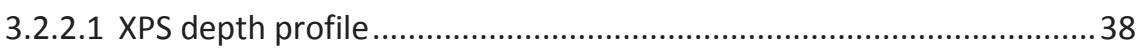

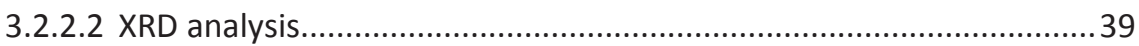

3.2.2.3 Laser Doppler Vibrometer measurements.................................... 40

3.2.3 Electric and ferroelectric characterization ........................................... 42

3.2.3.1 Polarization hysteresis measurements ......................................... 42

3.2.3.2 C-E and J-E measurements .................................................. 43

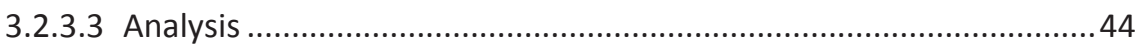

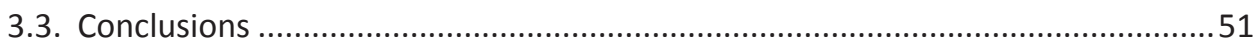

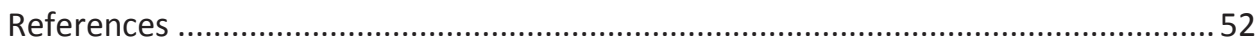

4. Fabrication and characterization of FinFETs ................................................55

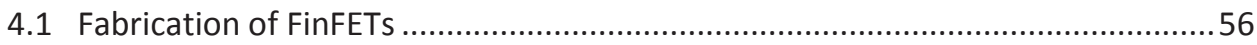

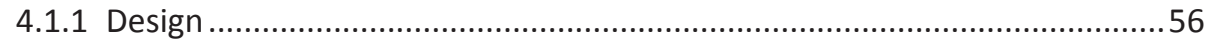

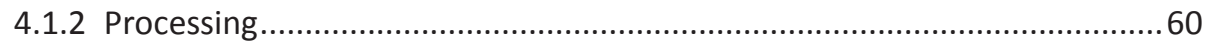

4.2 Physical and electrical characterization of FinFETs......................................65

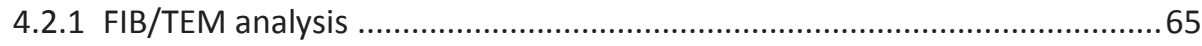

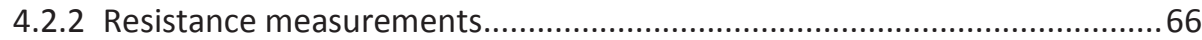

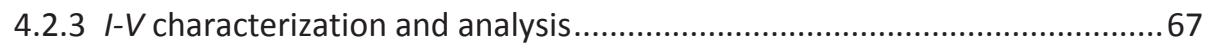

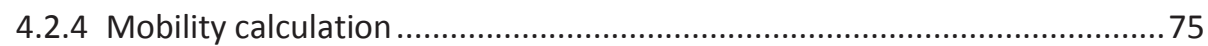

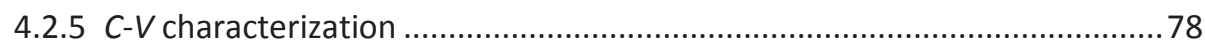

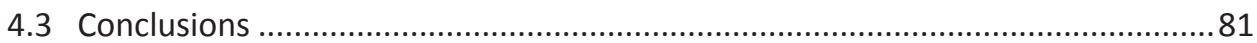

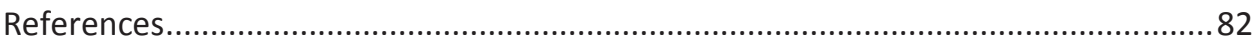

5. The PiezoFET: a piezoelectric layer around a FinFET .......................................85

5.1 The piezoelectric layer as a stressor .......................................................... 86

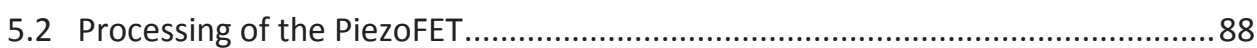

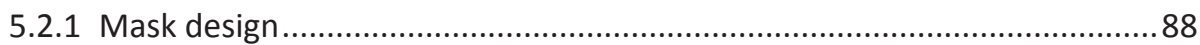

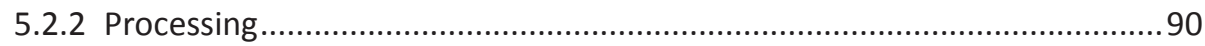

5.3 Physical, optical and ferroelectric characterization of PZT on FinFETs...............992

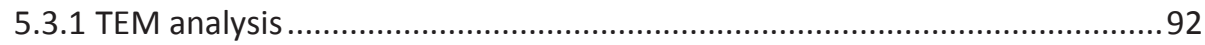


Contents

5.3.2 Laser Doppler vibrometer measurements .................................................. 94

5.3.3 Polarization hysteresis measurement .................................................... 96

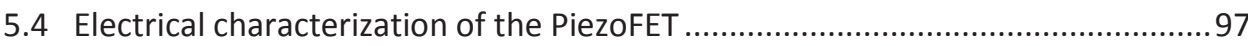

5.4.1 Effect of the PZT deposition on the $I_{\mathrm{d}}-V_{\mathrm{gs}}$ curves ..................................... 97

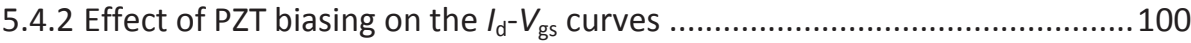

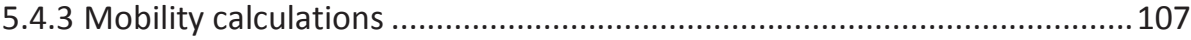

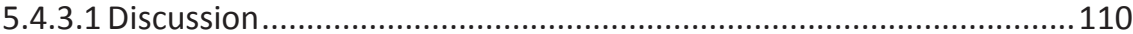

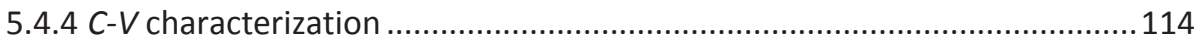

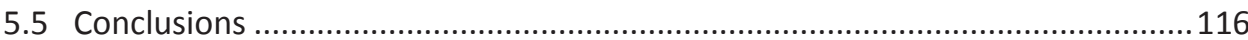

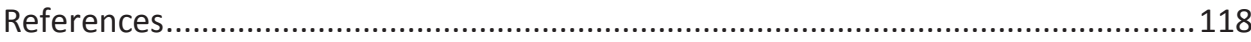

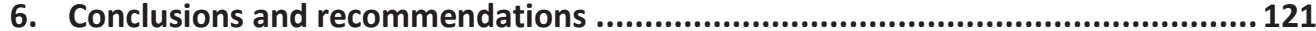

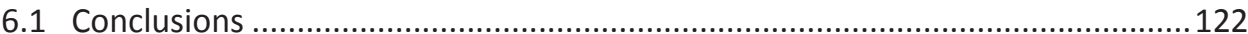

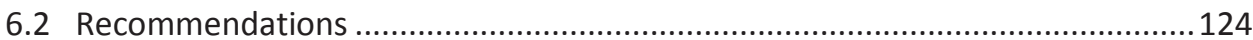

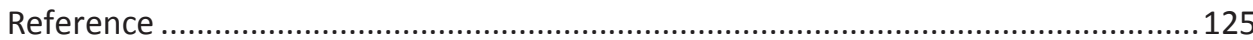

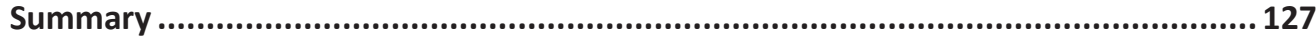

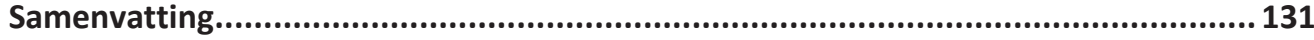

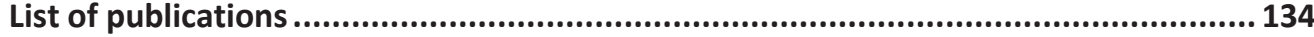

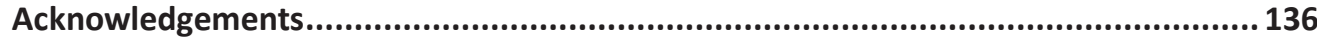




\section{Introduction}

This chapter presents a general introduction to this thesis. The motivation, the project description and objectives are described. The reasoning behind the preferred device configuration and materials are discussed. Furthermore, concepts of steep subthreshold devices reported from literature are summarized and the effects of strain in the Si channel are addressed. This chapter ends with an outline of the thesis. 


\subsection{The PiezoFET project}

The official title of the project leading to this thesis is "Piezoelectric layer formation on a silicon channel". It basically considers a piezoelectric layer on top of a fin shaped fieldeffect transistor (FinFET) channel. "PiezoFET" is the name referring to the fabricated device structure in this thesis. The described project is funded by the Dutch Technology Foundation (STW), with project number 10176. During the given period of time, the feasibility of this novel device structure with a piezoelectric stressor has been investigated. This work has been conducted at the MESA+ Institute for Nanotechnology, The Netherlands.

\subsection{Concepts for steep sub-threshold slope FETs}

The parameter that quantifies the electrostatic gate control over the sub-threshold drain current $\left(I_{\mathrm{d}}\right)$ of a FET is called the sub-threshold swing (SS). The SS is, by definition, the change in gate voltage resulting in one order of magnitude change in the drain current, i.e. the inverse of the sub-threshold slope. It is presented in Fig. 1.1 (no strain).

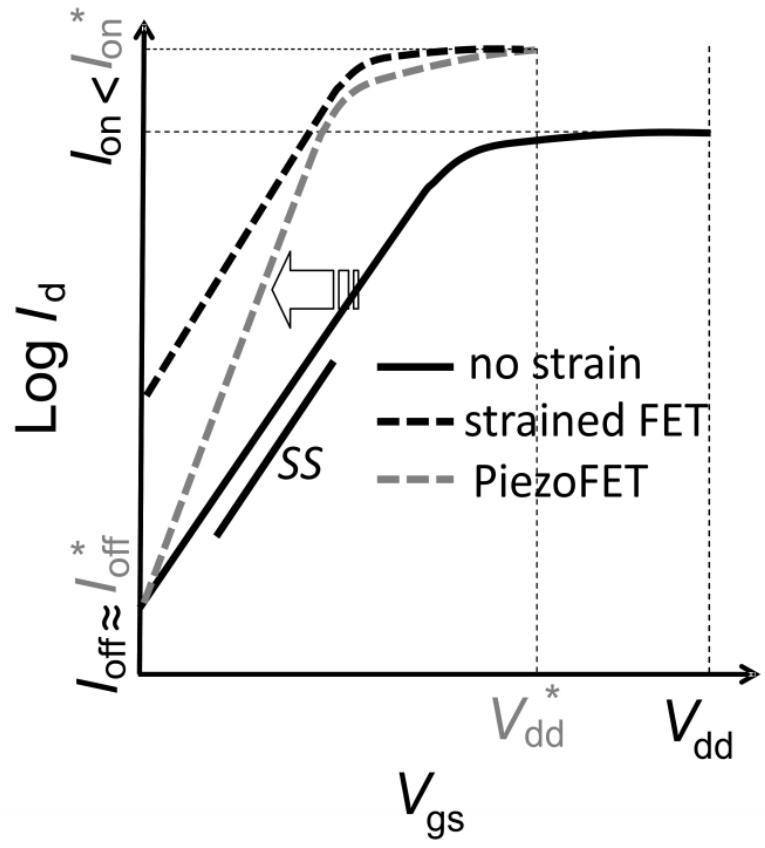

Fig. 1.1 Illustration of the $I_{\mathrm{d}}-V_{\mathrm{gs}}$ characteristics of a MOSFET on semi-logarithmic scale. The characteristics are shown for a device with no strain (i.e. relaxed condition), constant strain and strain formed by the converse piezoelectric effect (i.e. the PiezoFET). The sub-threshold swing (SS) is also indicated. 
For an ideal Si transistor, i.e. there is good electrostatic gate control over $I_{\mathrm{d}}$, the $S S$ equals $60 \mathrm{mV} / \mathrm{dec}$ at room temperature. In practice this can be obtained for (long channel) fully depleted FinFETs and ultra-thin body SOI MOSFETs where the depletion capacitance is negligible, as will be discussed in chapter 4 . Good gate control is important since it reduces the sub-threshold current which in turn determines the leakage power loss [1], an important contribution to the total power loss in a chip. Since, in advanced CMOS technology, there is a continuous trend in increasing functionality and speed of the electronic chip, the power consumption of the transistors should be reduced even more.

For CMOS, the so-called strain, i.e. mechanical deformation, is employed in silicon both by the academia and industry to increase the performance at a lower power. The conventional type of strain is typically constant depending on the surrounding materials and leads to a relatively high off-current $\left(I_{\text {off }}\right)$ and on-current $\left(I_{\text {on }}\right)$ provided all other process parameters have been kept the same [2-4], as illustrated in Fig. 1.1 (strained FET).

As discussed in chapter 2, the sub-threshold current is exponentially dependent on the band alignment, which is affected by strain formation and leads to an increased $I_{\text {off. }}$ This strain-induced change in band alignment could also lead to increased charge carrier mobility values resulting in a higher $I_{\text {on }}$. Fig. 1.2 summarizes the effect of the stress components on the mobility in FinFETs (redrawn after [5]). It can be concluded from this figure that, since n-FinFETs are fabricated in this thesis, biaxial tensile stress along the channel length and fin width is favorable.

However, as illustrated in Fig. 1.1 a SS of less than $60 \mathrm{mV} / \mathrm{dec}$, i.e. a sub-thermal subthreshold swing, is desirable. Several device concepts have been proposed to obtain a sub-thermal SS. Tunnel FETs and impact ionization MOSFETs have been reported for this purpose [6-10]. However, there are some challenges like a low on- to off-current ratio $I_{\text {on }} / I_{\text {off }}$ and hot carrier effects. In addition, negative capacitance FETs have been demonstrated to decrease the SS by replacing the standard insulator with a ferroelectric insulator [11, 12]. Therefore, before being successfully embedded in the CMOS technology, the gate stack materials and theory need to be studied further.

In this work, we propose and realize a new device concept which makes use of the converse piezoelectric effect to form a strain modulation in a silicon ( $\mathrm{Si}$ ) body. The basic idea behind this concept is that the strain in the Si body is increasing for a higher bias over the piezoelectric layer; that is placed on top of or around a Si body. This device concept is called the PiezoFET. The ideal $I_{\mathrm{d}}-V_{\mathrm{gs}}$ characteristic is presented in Fig. 1.1 (PiezoFET).

In the PiezoFET, strain arises due to an upward and downward motion of the piezoelectric layer caused by an external bias. The strain is basically zero in the off-state, meaning that the leakage current does not change compared to a relaxed condition, while in the on- 
state, by biasing the piezoelectric layer, a maximum amount of strain is obtained [13]. Consequently, the SS can be reduced by a piezoelectric stressor.

The resulting strain condition is not straightforward since it strongly depends on the (electro-) mechanical properties and layer thicknesses of the surrounding materials. The device geometry is equally important since it determines the (electro-) mechanical boundary conditions. These are different for a planar structure and a 3D structure like a FinFET. In Fig. 1.3 a schematic lay out of an SOI FinFET is presented, showing the Si fin, source and drain and gate configuration on a buried oxide (BOX) layer. Here the fin width, distance between fins and the aspect ratio are important. It is expected that for a high aspect ratio and relatively small fin (and pitch) dimensions strain can be obtained in a FinFET-based PiezoFET. In this case, a piezoelectric layer and electrodes are introduced in the structure to control strain. More analysis on the strain induced by the piezoelectric layer is discussed in chapter 5 .

By considering all the strain related effects that have been mentioned until now, the change in the mobility and $I_{\mathrm{d}}-V_{\mathrm{gs}}$ characteristics can be estimated. The ultimate goal of the piezoelectric effect induced strain on the $I_{\mathrm{d}}-V_{\mathrm{gs}}$ characteristics is illustrated in Fig. 1.1 (PiezoFET).

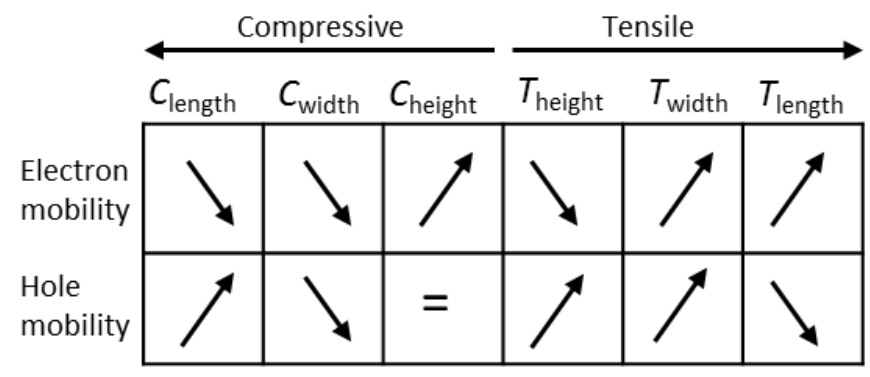

Fig. 1.2 The effect of stress components on the $(110) /[1 \overline{1} 0]$ FinFET electron and hole mobility. Redrawn after [5].

In summary, it is expected that the PiezoFET can offer a great potential as a low-power switch. In this study, the PiezoFET in a fin-shaped field-effect transistor (FinFET) configuration has been realized and analyzed.

\subsection{Choice of a device configuration and materials}

\subsubsection{FinfETs as a replacement of MOSFETs}

Planar bulk transistors have been serving the needs for integrated circuits for several decades. Over the years, the size of the transistors has been decreasing following 
Moore's law [14]. The motivation is saving space and reducing the unit cost. An increase in switching speed (due to shrinking the size of the gate) is a useful by-product of the miniaturization. However, continuous downscaling of these devices leads to an increase in short channel effects (SCE). SCE dominates when channel lengths are comparable to depth of source and drain junctions and depletion widths. It causes a degradation of the sub-threshold characteristics and results in higher standby power consumption [15]. After the introduction by Hisamoto et al. [16], the fin-shaped field-effect transistor (FinFET) has attracted much interest to solve this issue. A schematic view of the FinFET with a single fin on a silicon-on-insulator (SOI) substrate is shown in Fig. 1.3.

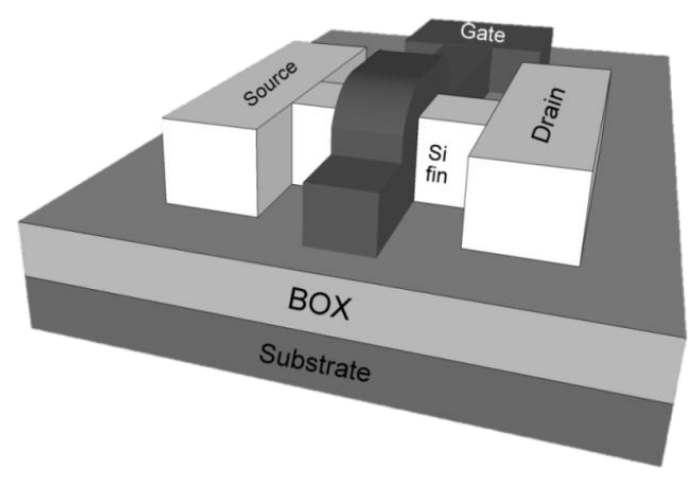

Fig. 1.3. Schematic layout of an SOI FinFET, bird's eye view.

The FinFET is a potential replacement of the bulk MOSFET. It comprises a gate around silicon (Si) body and depending on its design, it is considered as a double-gate or a triplegate (this figure) device. With the help of the double-gate or triple-gate architecture, the FinFET enables a better electrostatic gate control over the channel, which comes with relatively low power consumption in standby mode $[17,18]$. FinFETs are already a part of the most modern processors.

Furthermore, if the fin body was designed ultra-narrow, it would be entirely depleted enabling volume inversion [19]. The same architecture can also be obtained with a planar SOI FET having an ultra-thin silicon body. A fully depleted body with low doping helps i) to obtain an inverse sub-threshold slope (i.e. subthreshold swing, SS) close to the theoretical value of $60 \mathrm{mV} / \mathrm{dec}$ at $T=300 \mathrm{~K}$ (this will be discussed in section 1.3), ii) to tailor the threshold voltage $\left(V_{\text {th }}\right)$ by adjusting the work function of the gate material only, iii) to reduce the impurity scattering thereby increasing the mobility and iv) to eliminate random dopant fluctuations [20-22]. In addition, since the charge carriers are confined in the body, the effects related to surface imperfections, such as traps, and scattering are less important for ultra-narrow fins. This means that the switching speed and mobility can be increased [23]. However, when the fin width is too small, due to the quantum confinement effect, carrier scattering inside the channel becomes more important than 
surface scattering; resulting in a reduced mobility. Therefore, optimum conditions need to be found regarding the device architecture.

Along with its superior properties, the development of the FinFET leads to design and process related requirements like $i$ ) the threshold voltage adjustment, which requires gate work function tuning around the whole fin, ii) fringing capacitance between gate and top/bottom of the source/drain (S/D) regions, which requires minimizing fin pitch and using merged $S / D$, iii) parasitic $S / D$ series resistance, which requires epitaxially raised $S / D$ and silicide engineering and $i v$ ) performance variability, which requires uniform fin width and gate work function [24-28]. Therefore, the design parameters need to be considered to eliminate or to minimize these effects.

In this work, along with all the advantages mentioned above, the choice of the FinFET-like structure for the PiezoFET with narrow fins is a good candidate to obtain the optimum amount of strain in the Si channel.

FinFETs can be fabricated on SOI or bulk substrates. Up to date, there have been several reports mentioning the advantages of SOI FinFETs over their bulk counterparts [29-31]. Although they are more expensive, SOI substrates and lowly doped fins result in less junction capacitance, high mobility and reduced mismatch [32]. Although self-heating effect is a known problem for SOI devices [33], it will not be an issue for low power device concepts.

In this research, SOI substrates were used to realize FinFETs. The SOI substrates were obtained from NXP Semiconductors, Nijmegen. They were first laser cut from $125 \mathrm{~mm}$ to $100 \mathrm{~mm}$ to make them compatible with the available systems in the MESA+ Nanolab. More details about the processing and analysis of the fabricated devices are given in chapter 4.

\subsubsection{Encapsulated TiN as a gate material}

In low power CMOS, a symmetrical and low threshold voltage metal gate is desired for $\mathrm{n}$ channel and p-channel devices [34, 35]. For many years poly-Si has been used as a gate material. The main reason for this is that a heavily doped poly-Si gate can be tailored such that it has work function close to the band edges (i.e. $\approx 4.2 \mathrm{eV}$ for $\mathrm{n}$-type and $\approx 5.2 \mathrm{eV}$ for p-type). Its work function can be further modulated by adjusting the doping conditions. This makes it suitable for both $\mathrm{n}$ - and $\mathrm{p}$-type devices. Another advantage of the poly-Si gate is the higher temperature budget. However, as a downside, the poly-depletion effect (i.e. creation of depletion layer in the gate, which adds in series to the oxide capacitance) might increase the effective oxide thickness, thereby decreasing the series capacitance and the drive current. In addition, the $\mathrm{p}+$ poly $\mathrm{Si}$ gate is not compatible with high- $k$ 
dielectrics due to Fermi level pinning [36]. Furthermore, the gate sheet resistance of even a heavily doped poly-Si is higher than that of a metal gate. The formation of silicide is used to increase the conductivity to solve this issue. For reducing the gate leakage and not to suffer from partial depletion effects, high- $\kappa$ materials are co-integrated with the gate technology in semiconductor industry.

The use of a metal gate eliminates these poly depletion and conductivity problems. However, in general, most metals are not compatible with high temperature processing (>600 ${ }^{\circ} \mathrm{C}$ ). Metal nitrides (in our case TiN), on the other hand, are more suitable for higher temperatures. TiN is encapsulated with a poly-Si layer for planarization purposes and to prevent oxidation. The poly-Si layer also serves as a hard mask for ion implantation. The work function of metal nitrides typically is between $4.4 \mathrm{eV}$ and $4.7 \mathrm{eV}$. This work function is not suitable for low power planar MOSFETs (it needs to be close to the Si band edge for planar structures) but they can be utilized for low power, multigate CMOS applications [37]. Therefore, in this study, we choose the poly-Si/TiN stack as a gate material for the fabricated SOI FinFET devices, using thermal $\mathrm{SiO}_{2}$ as the gate dielectric.

\subsubsection{Converse piezoelectric effect as a stressor}

When an electric field is applied to a piezoelectric material, the structure of the piezoelectric material is deformed mechanically by the converse piezoelectric effect [38]. This deformation will be transferred to the surrounding material. The electrical signal is then transformed into a mechanical or acoustic signal. The use of the converse piezoelectric effect has been reported before for different device structures.

In a bulk acoustic wave or a surface acoustic wave resonator, for instance, the converse piezoelectric effect is used to obtain a resonance [38, 39]. A report from Shayegan et al. [40] demonstrated the use of piezoelectric material to apply a uniaxial stress in a plane of a confined 2D electron system formed by an AlAs quantum well. In this way, the electronic properties of the $2 \mathrm{D}$ electron system can be modified.

Note that, the piezoelectric effect is widely used in III-V heterojunction device structures. In these devices, lattice mismatch induced strain causes piezoelectric polarization, which results in an increased electron concentration in a 2-dimentional electron gas (2DEG) and a threshold voltage shift $[41,42]$. However, to the best of our knowledge there has not been any experimental evidence on the electrical characteristics in these III-V devices caused by the opposite effect, hence the converse piezoelectric effect.

In this study, the converse piezoelectric effect is used for stressing Si FinFET channels [13]. The main objective is to change the transport properties to obtain high mobility and steep 
sub-threshold slope devices. Analyses about the strain effect in Si channels are given in chapter 2.

\subsection{Technological requirements and research questions}

As mentioned before, the objective of this thesis is to realize strained FinFETs with a piezoelectric stressor. There are quite some technological problems:

i) Fabricating the near-ideal FinFET device structures comprising narrow fin widths with a high aspect ratio to obtain a high amount of strain. This also implies that a combined effort of e-beam lithography and photolithography is required in the MESA+ cleanroom, which is not straightforward,

ii) Growing or depositing high quality, uniform piezoelectric material on top of the FinFET structures,

iii) Optimizing the thickness of the layers between the piezoelectric stressor and the Si channel to a minimum to prevent stress relaxation while maintaining the quality of these layers,

iv) Integration of the piezoelectric layer without degrading the Si channel.

As a result, many research questions arise:

i) What is the step coverage of the piezoelectric layer around the fin? What is its grain/domain orientation?

ii) Do we maintain good ferroelectric or piezoelectric properties for aggressively scaled dimensions for both the buffer and the piezoelectric layers?

iii) Can we integrate the piezoelectric layers with Si? This is especially difficult for good piezoelectric materials such as Lead zirconate titanate (PZT). Is there diffusion of contaminants from the PZT layer into the Si body? And, if so, do they affect the device behavior?

iv) What is the effect of the piezoelectric layers on the electrical characteristics of the FinFET? For instance, does it influence the SS or mobility as expected? Does it depend on the device dimensions and geometry?

v) Is the converse piezoelectric effect feasible for significantly stressing the channel?

These subjects are addressed and most of the questions will be answered in the following chapters of this thesis.

\subsection{Organization of the thesis}

This thesis is organized as follows: 
Chapter 2 introduces the use of strain in Si based devices. Strain formation and its effects on the transport properties such as band gap and mobility are discussed. More specifically, FinFETs with a constant strain are presented. Analysis of the strain in these devices has been performed with the help of electrical and optical characterization techniques. The results provide a clear evidence of the effect of strain on the band alignment of Si.

Chapter 3 introduces an experimental study on the sub-100 $\mathrm{nm}$ ferroelectric thin film PZT capacitors. The influence of the film thickness and the encapsulated TiN bottom electrode on the ferroelectric and piezoelectric properties of PZT has been investigated. Some important figures-of-merit have been obtained with the help of electrical and optical characterization techniques. These figures have been utilized to optimize the PiezoFET design in this thesis.

Chapter 4 presents the realization and characterization of FinFETs. FinFETs with different fin widths and channel lengths have been realized to obtain a variable strain configuration after the piezoelectric layer deposition. The presented devices show good electrical characteristics and they provide a basis for the novel device structure called the PiezoFET, in chapter 5 .

Chapter 5 describes the novel PiezoFET. It starts with a brief analysis of the piezoelectric layer as a stressor. Details of the device fabrication and characterization are presented. The observed effects in the transport properties of the PiezoFET have been investigated and are discussed. Finally, conclusions are given based on the analysis of the experimental results.

Finally, chapter 6 summarizes this study and presents recommendations for future research.

\section{References}

[1] A. P. Chandrakasan, S. Sheng, and R. W. Brodersen, "Low-power CMOS digital design", IEEE Journal of Solid-State Circuits, 27, pp. 473-484, 1992.

[2] K. Rim, S. Koester, M. Hargrove, J. Chu, K.A Jenkins, P. Mooney, J. Ott, T. Kanarsky, P. Ronsheim, M. leong, and H. S. Wong, "Strained Si NMOSFETs for high performance CMOS technology", IEEE Symposium on VLSI Technology, Digest of Technical Papers, pp. 59-60, 2001.

[3] J. G. Fiorenza, G. Braithwaite, C. Leitz, M. T.Currie, Z. Y. Cheng, V. K. Yang, T. Langdo, J. Carlin, M. Somerville, A. Lochtefeld, H. Badawi, and H. M. T. Bulsara. "Investigation of misfit dislocation leakage in supercritical strained silicon MOSFETs", Proceedings of 42nd annual IEEE International Reliability Physics Symposium pp. 493-497, 2004.

[4] T. Khrishnamohan, "Physics and Technology of High Mobility, Strained Germanium Channel Heterostructure MOSFETs" Ph.D. thesis, 2006.

[5] N. Serra, F. Conzatti, D. Esseni, M. De Michielis, P. Palestri, L. Selmi, S. Thomas, T. E. Whall, E. H. C. Parker, D. R. Leadley, L. Witters, A. Hikavyy, M. J. Hytch, F. Houdellier, E. Snoeck, T. J. Wang, W. C. Lee, 
G. Vellianitis, M. J. H. van Dal, B. Duriez, G. Doornbos, and R. J. P. Lander, "Experimental and physicsbased modeling assessment of strain induced mobility enhancement in FinFETs", IEEE International Electron Devices Meeting (IEDM) Technical Digest, pp. 1-4, 2009.

[6] O. M. Nayfeh, C. N. Chleirigh, J. L. Hoyt, and D. A. Antoniadis, "Measurement of Enhanced GateControlled Band-to-Band Tunneling in Highly Strained Silicon-Germanium Diodes", IEEE Electron Device Letters, 29, pp. 468-470, 2008.

[7] K. Gopalakrishnan, P. B. Griffin, and J. D. Plummer, "Impact ionization MOS (I-MOS)-Part I: device and circuit simulations" IEEE Transactions on Electron Devices, 52, pp. 69-76, 2005.

[8] K. Gopalakrishnan, R. Woo, C. Jungemann, P. B. Griffin and J. D. Plummer, "Impact ionization MOS (IMOS)-Part II: device and circuit simulations" IEEE Transactions on Electron Devices, 52, pp. 77-83, 2005.

[9] A. Seabaugh, and Q. Zhang, "Low voltage tunnel transistors for beyond-CMOS logic," Proc. of IEEE, 98, pp. 2095-2110, 2010

[10] A. M. Ionescu, "Nanoelectronics: Ferroelectric devices show potential", Nature Nanotechnology, 7, p. $83-85,2012$.

[11] S. Salahuddin, and S. Datta, "Use of Negative Capacitance to Provide Voltage Amplification for Low Power Nanoscale Devices", Nano Lett., 8, pp. 405-410, 2008.

[12] A. Rusu, G. A. Salvatore, D. Jimenez, and A. M. Ionescu, "Metal-Ferroelectric-Meta-Oxidesemiconductor field effect transistor with sub-60mV/decade subthreshold swing and internal voltage amplification", IEEE International Electron Devices Meeting (IEDM) Technical Digest, 2010.

[13] T. van Hemert, and R. J. E. Hueting, "Active Strain Modulation in Field Effect Devices", Proc. of the European Solid-State Device Research Conference (ESSDERC), pp. 125-128, 2012.

[14] G. Moore, "Cramming more components onto integrated circuits", Electronics Magazine, 381998.

[15] S. Dhar, M. Pattanaik, and P. Rajaram "Advancement in Nanoscale CMOS Device Design En Route to Ultra-Low-Power Applications" VLSI Design, 2011, id:178516, 2011.

[16] D. Hisamoto, W. C. Lee, J. Kedzierski, E. Anderson, H. Takeuchi, K. Asano, T. J. King, J. Bokor, and C. Hu, "A folded channel MOSFET for deep sub tenth micron era", IEEE International Electron Devices Meeting (IEDM) Technical Digest, pp. 1032-1034, 2008.

[17] X. Wu, F. Wang, and Y. Xie, "Analysis of Subthreshold FinFET Circuits for Ultra-Low Power Design", IEEE SOC conference, pp. 91-92, 2006.

[18] R. Vaddi, R. P. Agarwal, S. Dasgupta, and T. T. Kim, "Design and Analysis of Double-Gate MOSFETs for Ultra-Low Power Radio Frequency Identification (RFID): Device and Circuit Co-Design", J. Low Power Electron. Appl., 1, pp. 277-302, 2011.

[19] F. Balestra, S. Cristoloveanu, M. Benachir, J. Brini, and T. Elewa, "Double-gate silicon-on-insulator transistor with volume inversion: A new device with greatly enhanced performance" IEEE Electron Device Letters, 8, pp. 410-412, 1987.

[20] P. Yeh, and J. Fossum, "Physical sub-threshold MOSFET Modelling Applied to Viable Design of DeepSubmicrometer Fully Depleted SOI Low Voltage CMOS Technology", IEEE Transactions on Electron Devices , 42, pp.1605-1613, 1995.

[21] Y. Taur, "Analytic Solutions of Charge and Capacitance in Symmetric and Asymmetric Double-Gate MOSFETs", IEEE Transactions on Electron Devices, 48, pp. 2861-2869, 2001.

[22] H. Tuinhout, "Impact of Parametric Fluctuations on Performance and Yield of Deep-submicron Technologies", Proc. of the European Solid-State Device Research Conference (ESSDERC), pp. 95-102, 2002.

[23] M. M. Chowdhury, and J. G. Fossum, "Physical insights on electron mobility in contemporary FinFETs", IEEE Electron Device Letters, 27, pp. 482-485, 2006.

[24] V. Narendar, R. Mishra, S. Rai, Nayana R and R. A. Mishra, "Threshold voltage control schemes in FinFETs" International Journal of VLSI design \& Communication Systems (VLSICS), 3, pp.175-191, 2012. 
[25] C. R. Manoj, A. B. Sachid, F. Yuan, C.-Y.Chang, and V. R. Rao, "Impact of Fringe Capacitance on the Performance of Nanoscale FinFETs" IEEE Electron Device Letters, 31, pp. 83-85, 2010.

[26] A. Dixit, A. Kottantharayil, N. Collaert, and M. Goodwin, "Analysis of the Parasitic S/D Resistance in Multiple-Gate FETs", IEEE Transactions on Electron Devices, 52, pp.1132-1140, 2005.

[27] A. Sakic, M. Poljak, V. Jovanovic, and T. Suligoj, "Influence of Scaling and Source/Drain Series Resistanceon the Characteristics of Ultra-Thin Body FinFETs", Proceedings of the 31st International Convention MIPRO, 2008.

[28] T. Matsukawa, S. O'uchi, K. Endo, Y. Ishikawa, H. Yamauchi, Y. X. Liu, J. Tsukada, K. Sakamoto and M. Masahara, "Comprehensive Analysis of Variability Sources of FinFET Characteristics", IEEE Symposium on VLSI Technology Digest of Technical Papers, pp. 118-119, 2009.

[29] M. Poljak, V. Jovanovic, and T. Suligoj, "Improving bulk FinFET DC performance in comparison to SOI FinFET”, Microelectric Engineering, 69, pp. 2078-2085, 2009.

[30] T. Chiarella, L. Witters, A. Mercha, C. Kerner, M. Rakowski, C. Ortolland, L.-A. Ragnarsson, B. Parvais, A. De Keersgieter, S. Kubicek, A. Redolfi, C. Vrancken, S. Brus, A. Lauwers, P. Absil, S. Biesemans, and T. Hoffmann "Benchmarking SOI and bulk FinFET alternatives for PLANAR CMOS scaling succession", Solid-State Electronics, 54, pp. 855-860, 2010.

[31] J. G. Fossum, Z. Zhou, L. Mathew, and B. Y. Nguyen," SOl versus bulk-silicon nanoscale FinFETs", Solid State Electronics, 54, pp. 86-89, 2010.

[32] A. Chiarella, L. Witters, A. Mercha, C. Kerner, R. Dittrich, M. Rakowski, C. Ortolland, L. A. Ragnarsson, B. Parvais, A. De Keersgieter, S. Kubicek, A. Redolfi, R. Rooyackers, C. Vrancken, S. Brus, A. Lauwers, P. Absil, S. Biesemans, and T. Hoffmann, "Migrating from planar to FinFET for further CMOS scaling: SOI or bulk?", Proc. of the European Solid-State Device Research Conference (ESSDERC), pp. 84-87, 2009.

[33] W. Molzer, Th. Schulz, W. Xiong, R. C. Cleavelin, K. Schrüfer, A. Marshall, K. Matthews, J. Sedlmeir, D. Siprak, G. Knoblinger, L. Bertolissi, P. Patruno, J.-P. Colinge, "Self Heating Simulation of Multi-Gate FETs", Proc. of European Solid-State Device Research Conference (ESSDERC), pp. 311-314, 2006.

[34] S. J. Hillenius, R. Liu, G. E. Georgiou, R. L. Field, D. S. Williams, A. Kornblit, D. M. Boulin, R. L. Johnston, and W. T. Lynch, "A symmetric submicron CMOS technology", IEEE International Electron Devices Meeting (IEDM) Technical Digest, pp. 252-255, 1986.

[35] H. R. Huff, and D. C. Gilmer, "High Dielectric Constant Materials, VLSI MOSFET Applications", Springer, 2005.

[36] C. Hobbs, L. Fonseca, V. Dhandapani, S. Samavedam, B. Taylor, J. Grant, L. Dip, D. Triyoso, R. Hegde, D. Gilmer, R. Garcla, D. Roan, L. Lovejoy, R. Rai, L. Hebert, H. Tseng, B. White and P. Tobin, "Fermi Level Pinning at the PolySi/metal Oxide Interface", IEEE Symposium on Technology, Digest of Technical Papers, pp. 9-10, 2003.

[37] W. Xiong, C. R. Cleavelin, C.-H. Hsub, M. Ma, K. Schruefer, K. V. Armin, T. Schulz, I. Cayrefourcq, C. Mazure, P. Patruno, M. Kennard, K.Shin, X. Sun, T.-J. King Liu, K. Cherkaoui, and J. P. Colinge, "Intrinsic Advantages of SOI Multiple-Gate MOSFET (MuGFET) for Low Power Applications" ECS Transactions, 6, pp. 59-69, 2007.

[38] B. A. Auld, "Acoustic fields and waves in solids" $2^{\text {nd }}$ ed., Krieger Publishing Company, 1990.

[39] K. M. Lakin, G. R. Kline, and K. T. McCarron, "High-Q microwave acoustic resonators and filters", IEEE Trans. Microwave Theory and Techn., 41, pp. 2139-2146, 1993.

[40] M. Shayegan, K. Karrai, Y. P. Skolnikov, K. Vakili, E. P. De Poortere, and S. Manus, "Low-temperature, in situ tunable, uniaxial stress measurements in semiconductors using a piezoelectric actuator", Appl. Phys. Lett., 83, pp. 5235-5237, 2003.

[41] T. F. Kuech, R. T. Collins, D. L. Smith, and C. Mailhiot, "Field-effect transistor structure based on straininduced polarization charges", Journal of Applied Physics, 67, pp. 2650-2652, 1990.

[42] A. Vais, "Physical Simulation of GaN based HEMT", Master Thesis, Chalmers University of Technology, Sweden, 2012. 


\section{Strained silicon devices}

In this chapter, a brief introduction to strained silicon devices is given. The effect of strain on the band gap and mobility is discussed. The electrical and optical characterization of TiN induced strain on FinFETs is presented. It is demonstrated that the conduction band offset is changing depending on the fin dimensions, which can be explained by counteracting quantum confinement and strain effects. 


\subsection{Strain formation in silicon-based devices}

To improve the transport properties of a (crystalline) semiconductor material, and hence the device performance, so-called strain is often applied in the semiconductor technology and in research projects. Strain is defined as the deformation of a material normalized to its stress free dimension [1]. Strain causes elastic stress (or forces). The strain $(\varepsilon)$ and stress $(\sigma)$ are related according to the scalar notation $\sigma=E \varepsilon$, where $E$ is the stiffness or Young's modulus of the material. Basically, one can imagine the strain effect to be pushing atoms in the periodic crystal together (i.e. compressive or negative strain) or pulling them apart (i.e. tensile or positive strain). This can occur in one direction (uniaxial) only or in several directions (biaxial or triaxial).

There are different ways to form strain in silicon (Si). Some examples are phonon induced lattice vibrations, lattice mismatch during film growth, intrinsic strain in deposited thin films and externally applied strain.

The calculations of Bardeen and Shockley on deformation potentials and the measurements of Smith on the piezoresistance effect are the first physical reports explaining the enhancement of mobility due to strain [2, 3]. Since then, there have been many experimental and theoretical reports on the effect of strain on the band gap and charge carrier mobility [4-11]. Although there was a strong interest in using strained silicon-germanium ( $\mathrm{SiGe}$ ) alloys for high mobility channels, it could not have been realized in Si-based MOSFETs until 1991 due to the high density of interface states generated during oxidation. Iyer et al. [12] were the first who demonstrated a promising high-quality dielectric system by capping the SiGe with a thin Si layer followed by oxide deposition. Later on, Welser et al. [4] first demonstrated an improvement in device performance by strain in n-channel MOSFETs. Here, a strained-Si channel was grown on a relaxed silicongermanium $\left(\mathrm{Si}_{0.71} \mathrm{Ge}_{0.29}\right.$ ) layer. Because of the lattice mismatch between the relatively thick SiGe layer and thin Si layer, global biaxial tensile strain has been formed in the latter. In the following years, strained $\mathrm{Si} /$ relaxed $\mathrm{Si}_{1-x} \mathrm{Ge}_{x}$ systems have been studied extensively and have become a promising candidate for a performance boost in CMOS, mainly by enhancing the mobility [13-16] and consequently the on-current. In addition, some groups reported on the use of $\mathrm{Si}_{1-x} \mathrm{Ge}_{\mathrm{x}}$ systems in the source and drain regions acting as a local stressor in the channel region for improving the hole mobility in $p$ channel MOSFETs $[17,18]$. This concept is presently in production, for example, in Intel's $45 \mathrm{~nm}$ Xeon technology.

Along with $\mathrm{Si}_{1-\mathrm{x}} \mathrm{Ge}_{\mathrm{x}}$, silicon nitride $\left(\mathrm{SiN}_{\mathrm{x}}\right)$, titanium-nitride (TiN) and piezoelectric materials are also well-known examples for stressors utilized for different purposes. $\mathrm{SiN}_{\mathrm{x}}$ has been studied as an external and local stressor in many studies mainly as a capping layer in 
planar and 3D device structures [18-21]. The strain formed by TiN and piezoelectric layers in FinFETs will be discussed in sections 2.3 and 5.1, respectively.

\subsection{The effect of strain on the band gap and mobility}

Strain is known to change the mobility and modify the band gap of the material depending on the strain type, direction and the material's properties. It is important to note that both the conduction and valence band in Si are degenerate: the first comprises six so-called $\Delta$ valleys while the latter can be subdivided into a heavy hole $(\mathrm{HH})$ and a light hole (LH) band. When stress is applied, the degeneracy basically becomes less. The changes in conduction and valence band in bulk Si for biaxial tensile strain is shown in Fig. 2.1. Valence band in Fig 2.1 (b) is plotted in energy vs. reciprocal space (or k-space, with $k=2 \pi /$ lattice constant).

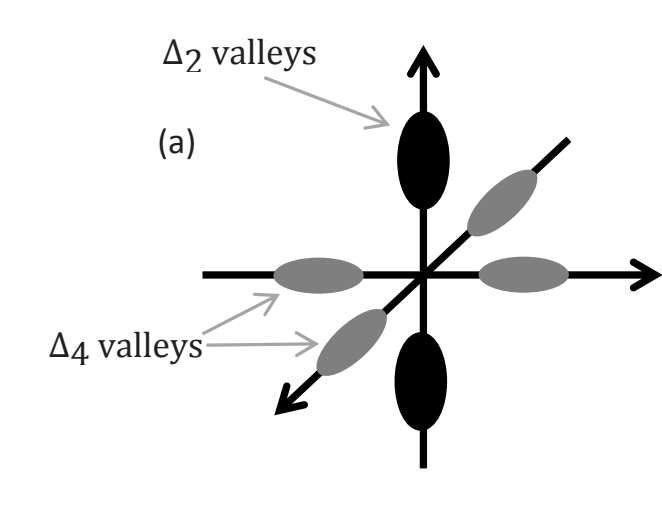

Bulk Si Strained Si
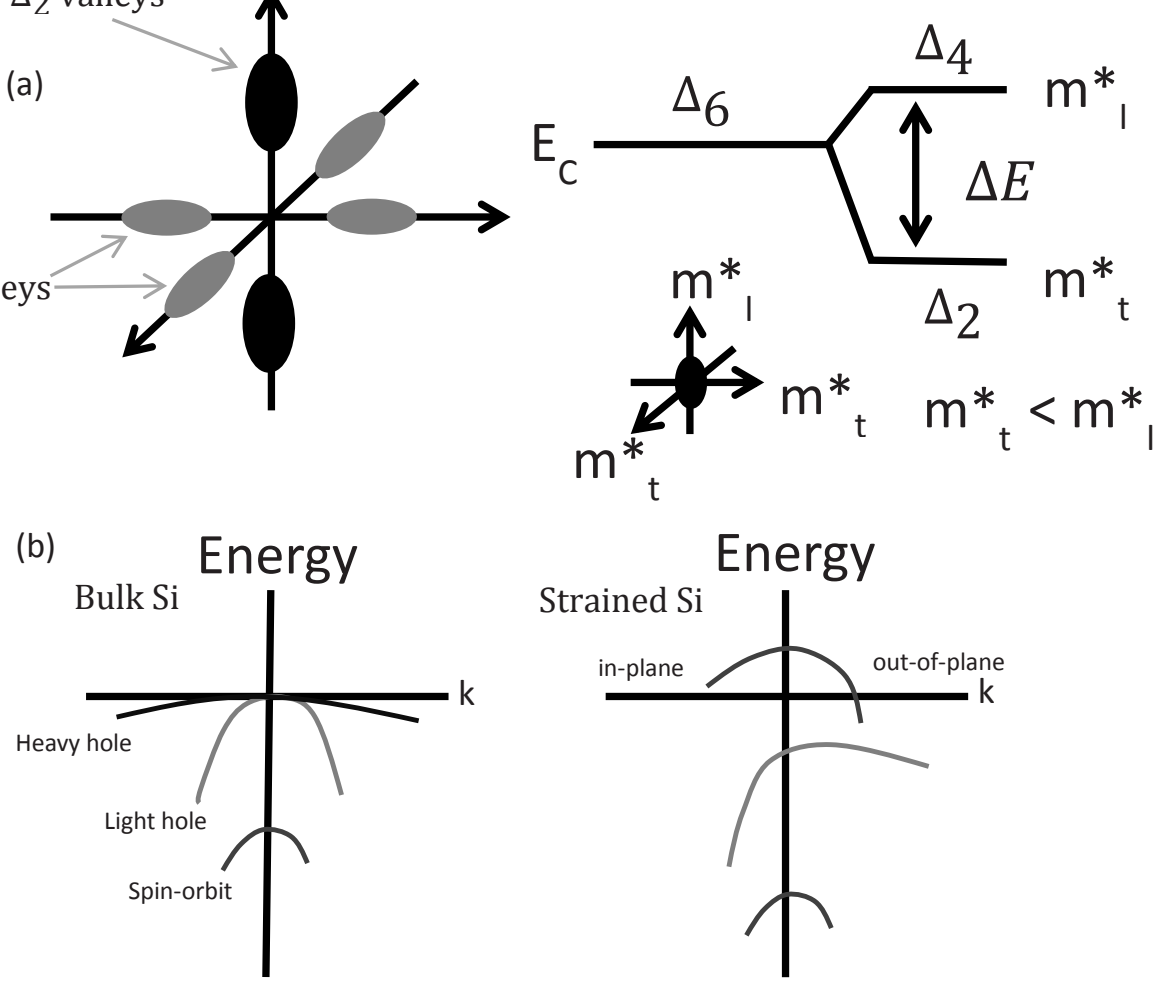

Fig. 2.1 Biaxial tensile strain induced changes in the (a) conduction band and (b) valence band of planar/bulk Si. Redrawn after Rim et al. [22].

The Si conduction band valleys split into $\Delta_{2}$ and $\Delta_{4}$ valleys depending on the amount and orientation (in this case [001] oriented) of stress or strain. On the other hand, the valence 
$\mathrm{HH}$ band shows a linear while the LH band shows a nonlinear dependency with strain. In case of a large amount of strain, the electrons completely populate the lower $\Delta_{2}$ valley [7]. Since the in-plane conductivity effective mass of the $\Delta_{2}$ valleys is smaller than that of the $\Delta_{4}$ valleys and intervalley scattering is suppressed due to this splitting, the electron mobility increases [23]. Similarly, strain lifts the degeneracy between $\mathrm{HH}$ and $\mathrm{LH}$ bands and the spin orbit band is lowered in energy resulting less intervalley scattering and improvement in-plane hole mobility. In fact, as indicated in Fig. 2.1 (a) and (b), the conduction band edge drops and valence band edge increases resulting in a reduced band gap.

Skotnicki et al. [6] reported the effect of strain on the band gap and mobility of a bulk MOSFET with a $<110>$ oriented channel. Table 2.1 shows a part of this report. The table indicates that the band gap decreases for all stress conditions. However, the net change depends on the presence of quantization effects. The change in mobility, on the other hand, depends on the stress condition. It is observed that tensile stress along the channel length is needed to obtain higher electron mobility. In contrast, it leads to a decrease in hole mobility. 
Table 2.1 Analysis of strain configuration and its effects in CMOS (Skotnicki et al. [6], with courtesy).

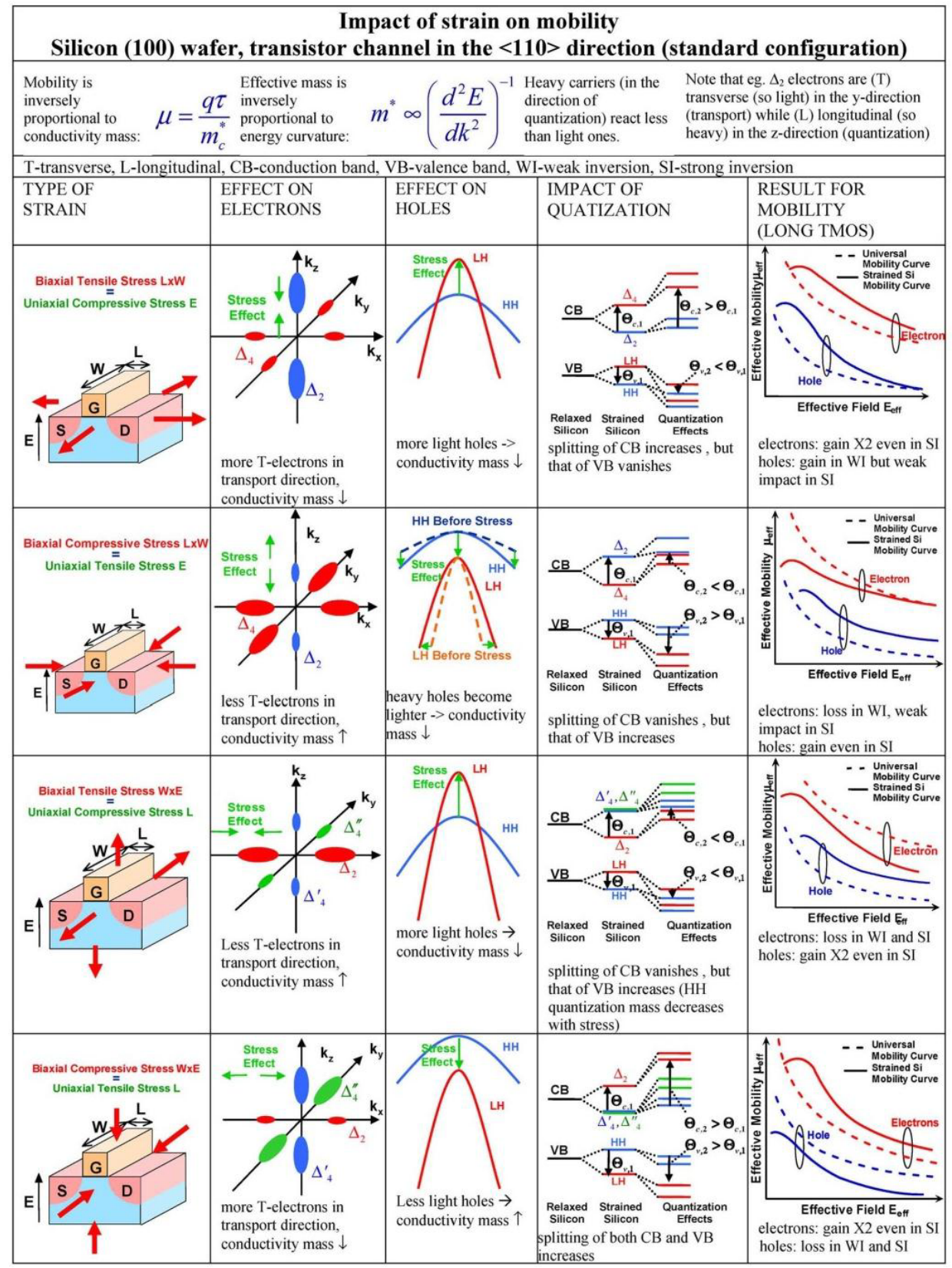

K. A. Rim et al. [24] experimentally showed for the first time that the sub-threshold current of an n-type Si MOSFET increases, or the threshold voltage drops ( by $\approx 200 \mathrm{mV}$ ), when biaxial tensile strain is applied. This is due to this smaller bandgap and lower conduction band in strained Si compared to the relaxed or bulk Si counterpart. In 
addition, calculations indicate that both biaxial and uniaxial tensile stress causes a band gap decrease for Si, Ge and GaAs [11]. However, for compressive stress, the band gap changes depending on the stress configuration.

The effect of strain on the mobility of n-MOSFET and p-MOSFET device structures under different stressing conditions has been studied extensively. Depending on the orientation, strain type and level, mobility enhancement was demonstrated in many reports. This helped to understand and to control the effects $[5,25,26]$.

Furthermore, the effects of strain on a material with a thickness of only several atomic layers differ from bulk material. Quantization effects have to be taken into account when dimensions are downscaling. In that case, the band structure and mobility will be affected depending on both the applied strain and quantization. With quantization, the apparent or effective band gap of the material increases [27-29]. This effect is also demonstrated and explained in section 2.3.1.1 for n-type FinFETs. Strain in these devices resulted in an enhancement in mobility and on-current as reported by Rim et al. [30] and Feste et al. [31]. They demonstrated a $110 \%$ and $230 \%$ improvement in electron mobility in a $7 \mathrm{~nm}$ thick planar strained Si layer and $42 \mathrm{~nm}$ thick, $\approx 35 \mathrm{~nm}$ wide strained Si fin, respectively. This resulted in a proportional increase in on-current of the strained FinFET. These figures show that strained FinFET structures are important for CMOS applications.

\subsection{TiN induced strain on FinFETs}

In section 2.1, several techniques of strain formation have been discussed. This section focusses on the external and local strain formation in FinFETs due to the use of a stiff conductor such as TiN [32-35]. The motivation of using the FinFET configuration was discussed in chapter 1 , section 1.3.1.

TiN induced strain in $\mathrm{Si}$ originates from its growth mechanism and the difference in coefficient of thermal expansion (CTE) between the TiN gate and the Si. This type of strain has been studied before [26, 36, 37] focusing on the mobility. In this work, FinFETs manufactured by NXP-TSMC Research Leuven, Belgium [38], have been studied focusing on the band alignment. The silicon-on-insulator (SOI) FinFETs have a (001) surface and (110) fin sidewall orientation. It is basically the same device set as reported by Serra et al. [37] which has been analyzed further. Fig. 2.2 shows a TEM cross section of a FinFET on an SOl substrate. The gate stack consists of a gate dielectric layer with a $1 \mathrm{~nm}$ thick silicon-dioxide $\left(\mathrm{SiO}_{2}\right)$ and $1.7 \mathrm{~nm}$ thick hafnium-silicate ( $\mathrm{HfSiO}$ ) layer and a $5 \mathrm{~nm}$ thick TiN layer (deposited by physical vapor deposition) as the gate material with a poly-Si capping. All the devices characterized in this work comprised five fins with various fin widths $(5 \mathrm{~nm}$ $\leq W_{\text {FIN }} \leq 1000 \mathrm{~nm}$ ) and gate lengths $\left(35 \mathrm{~nm} \leq L_{\text {gate }} \leq 10 \mu \mathrm{m}\right)$. The fin height or SOl thickness was $60 \mathrm{~nm}\left(H_{\mathrm{FIN}}\right)$. For more processing details, refer to [38]. 


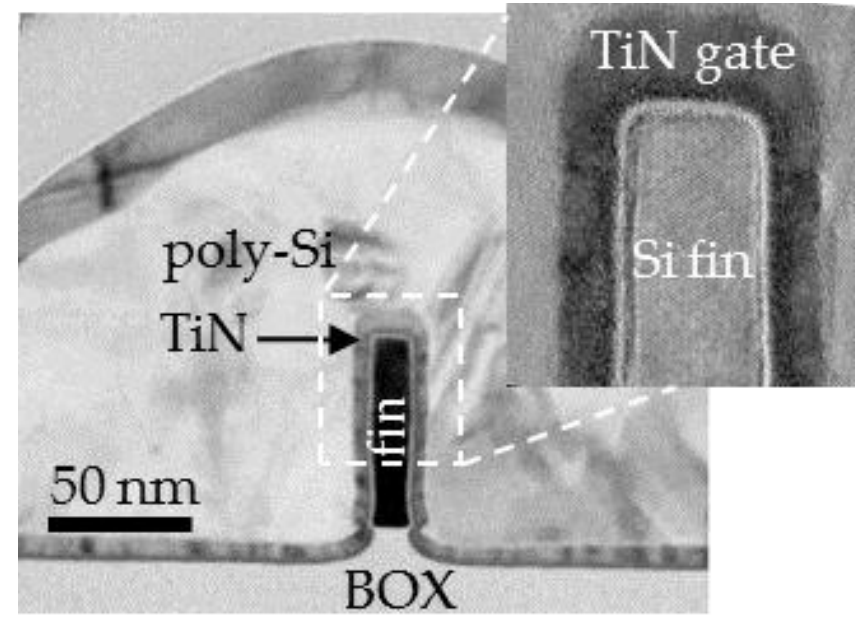

Fig. 2.2 TEM cross section picture of the FinFET (with courtesy of M. van Dal).

An important detail is the thermal cycle implemented after gate deposition. This results in a permanent strain in the channel [37]. This strain can be illustrated by simulations performed using a multiphysics Finite-element-method (FEM) tool (Comsol MultiPhysics 3.5a). In the simulations, the Si substrate was neglected to decrease the computational load. Fixed boundary conditions were applied below the buried oxide (BOX) layer to mimic the stiff Si substrate. For the Young's modulus and CTE of Si and TiN are taken 130 GPa \& $2.5 \times 10^{-6} \mathrm{~K}^{-1}$ and $600 \mathrm{GPa} \& 9.4 \times 10^{-6} \mathrm{~K}^{-1}$, respectively. The experimental 5-fin device structure was simplified to a single fin device. Symmetric boundary conditions were applied to the sides of the fins as well as at the source and drain regions. The rest of the boundaries were kept free to move. A high temperature cycle from $25^{\circ} \mathrm{C}$ to $1100{ }^{\circ} \mathrm{C}$ and back to $25^{\circ} \mathrm{C}$ has been implemented on the device structure to form strain as in the real case. During the thermal cycle of the stacked structure, the strain increases while the temperature increases. At elevated temperatures (above $1100{ }^{\circ} \mathrm{C}$ ) plastic relaxation occurs and the strain will be low. The simulations are referenced to this relaxed state. When the system is cooling down, the strain increases again and remains permanently in the Si body or fin. Figs. 2.3 (a)-(c) show the simulated vertical strain $\left(\varepsilon_{z z}\right)$ in the fin cross section of a $5 \mathrm{~nm}$ wide fin at $900{ }^{\circ} \mathrm{C}, 500{ }^{\circ} \mathrm{C}$ and $25{ }^{\circ} \mathrm{C}$, respectively. Compressive strain $\left(\varepsilon_{z z}\right)$ in the range of $-0.9 \%$ has been observed for a $5 \mathrm{~nm}$ fin width $\left(W_{\mathrm{FIN}}\right)$. The other strain components, $\varepsilon_{x x}$ and $\varepsilon_{y y}$ are much smaller than $\varepsilon_{z z}$ (up to $-0.18 \%$ ) and therefore neglected. The simulation for the $30 \mathrm{~nm}$ wide fin at $25^{\circ} \mathrm{C}$ is also depicted (Fig. 2.3 (d)) to show that the generated strain decreases when $W_{\text {FIN }}$ increases. It also becomes less uniform in the silicon body. FEM simulated maximum strain values are plotted against $W_{\text {FIN }}$ in Fig. $2.10^{1}$.

\footnotetext{
${ }^{1}$ The simulations were performed by Tom van Hemert, with courtesy.
} 
As demonstrated before by Serra et al. [37] compressive strain in the fin height direction favors an increase in electron mobility.

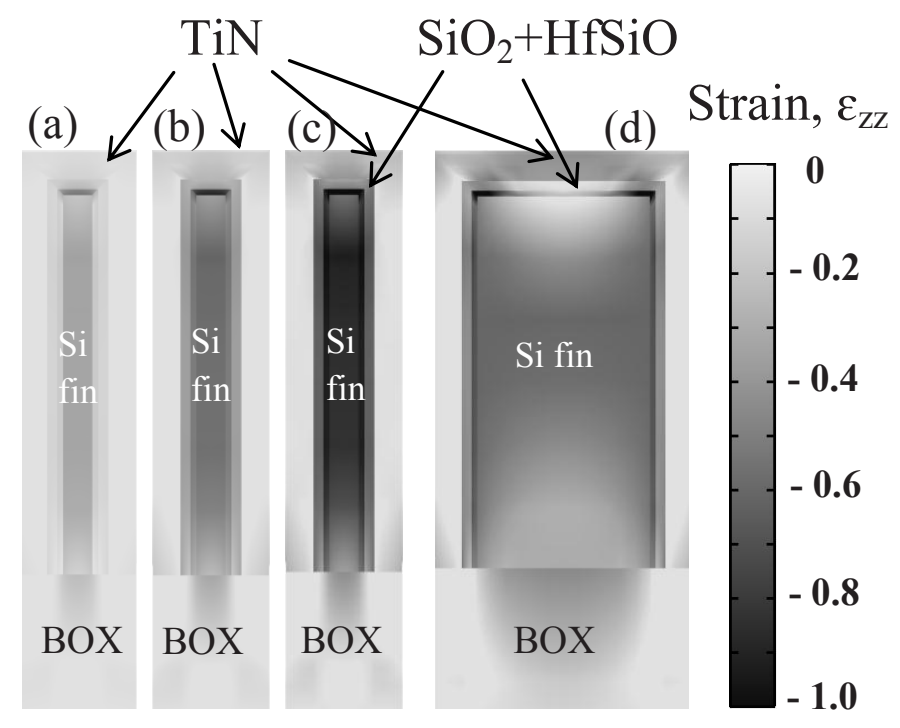

Fig. $2.3 \varepsilon_{z z}$ strain values of the $30 \mathrm{~nm}$ and $5 \mathrm{~nm}$ wide FinFETs obtained with multiphysics FEM simulations during cooling down cycle from $\mathrm{T}=1100{ }^{\circ} \mathrm{C}$ to $25^{\circ} \mathrm{C}$ for $W_{\mathrm{FIN}}=5 \mathrm{~nm}$ at (a) $900{ }^{\circ} \mathrm{C}$, (b) 500 ${ }^{\circ} \mathrm{C}$, (c) $25^{\circ} \mathrm{C}$, and (d) $25^{\circ} \mathrm{C}$ for $W_{\mathrm{FIN}}=30 \mathrm{~nm}$. The vertical bar indicates the strain values varying from 0 to -1.0 (compressive) strain. The thickness of the TiN film is $5 \mathrm{~nm}$ and the height of Si fin is 60 $\mathrm{nm}$. Note the relatively high strain value in the $\mathrm{HfSiO}$ gate dielectric.

\subsubsection{Characterization of strain by optical and its effects by electrical techniques}

Strain characterization plays an important role in understanding and controlling strain effects. There are different methods possible for this purpose. Raman spectroscopy [39, 40] is mainly used for strain characterization. However, holographic interferometry [37], synchrotron $x$-ray scattering [41] and nano-beam diffraction [42] techniques have also been used by several research groups. Each of these techniques has advantages and disadvantages regarding the resolution and sensitivity.

In addition to these direct measurement techniques, electrical characterization can also be employed to study the effect of strain in devices. In this case, strain effects are expected to appear as a change in the band gap and mobility as described in section 2.2. Using both optical and electrical characterization and in depth knowledge of strain and its effect can be obtained. In the following two sub-sections, I-V curves will be studied to analyze the effect of strain on FinFETs and Raman spectroscopy will be used to characterize the strain. 


\subsubsection{Temperature dependent $I-V$ measurements on strained devices}

Temperature dependent $I-V$ measurements were performed using $n$-type strained FinFET devices with different fin widths $\left(W_{\mathrm{FIN}}\right)$ and gate lengths $\left(L_{\text {gate }}\right)[32,34]$. By using the subthreshold current technique described by van der Steen et al. [43] energy band offsets of the FinFETs can be extracted. Since the band offsets depend on the amount of strain, this method can be utilized. As discussed before on our FEM simulations, the amount of strain in the FinFETs under study are expected to increase for a smaller $W_{\text {FIN }}$.

Valence or conduction band offsets can be calculated using the subthreshold current of the reference device having a wide fin (hence negligible strain), namely $I_{\text {ref }}$, and that of a device with a smaller fin width, $I_{\text {thin }}$. The ratio of subthreshold currents is then given by:

$$
\eta \equiv \frac{I_{\text {ref }}}{I_{\text {thin }}}=\frac{\mu_{\text {ref } \cdot g\left(W_{F I N, r e f}\right)}}{\mu_{\text {thin } . g\left(W_{F I N, t h i n}\right)}} \exp \left(\frac{\Delta E_{x}}{k T}\right)
$$

where $g\left(W_{\mathrm{FIN}}\right)$ represents the effective density of states (DOS) depending on the fin width, $\mu$ is the charge carrier mobility, and $\Delta E_{X}$ is the difference in conduction or valence band edge of the reference and narrow fin device. Since $\mathrm{n}$-type FinFETs have been studied for this purpose, only the conduction band offset has been determined. In order to determine the valence band offset $p$-type FinFETs are required. The temperature dependence of $\eta$ depends strongly on the conduction band offset because of the exponential term. Assuming equal temperature dependence of $\mu$ and DOS for both devices, $\Delta E_{x} / k$ is found to be equal to the slope of the $\ln (\eta)$ vs. $1 / T$. It is important to note that the strain changes with temperature. However, this change is small $(0.1 \%)$ compared to the overall strain (-0.6\%) [34].

I-V measurements of $(1 \overline{1} 0) /[110]$ oriented n-FinFET devices were done at temperatures ranging from $25^{\circ} \mathrm{C}$ to $150^{\circ} \mathrm{C}$. Since short channel effects can result in misinterpretation of the results, the conduction band offset was calculated from long channel devices (i.e. 1 $\mu \mathrm{m}$ and $10 \mu \mathrm{m})$. The maximum $W_{\text {FIN }}$ here was $30 \mathrm{~nm}$, which was used as a reference, and the minimum $W_{\text {FIN }}$ was $5 \mathrm{~nm}$. In Fig. $2.4 \ln (\eta)$ vs. $1 / T$ curves have been plotted for the FinFETs with $1 \mu \mathrm{m}$ and $10 \mu \mathrm{m}$ gate length. 

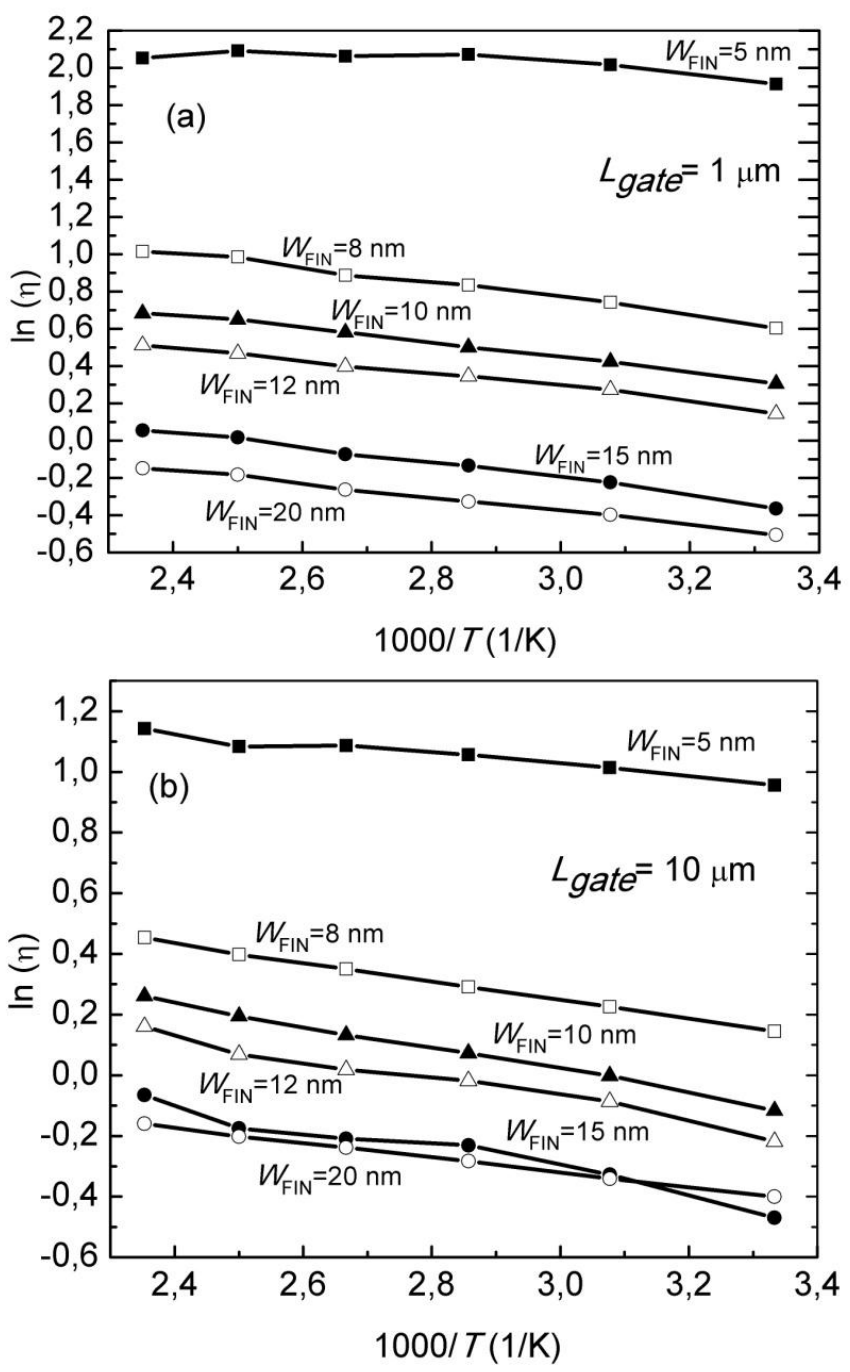

Fig. $2.4 \ln (\eta)$ vs. $1 /$ T curves of (a) $1 \mu \mathrm{m}$ and (b) $10 \mu \mathrm{m}$ long devices.

In Fig. 2.4 the $\ln (\eta)$ value increases for narrow fins. This effect is due to a decrease in mobility for very narrow fins [44]. However, this increase is stronger for a $1 \mu \mathrm{m}$ device than that of a $10 \mu \mathrm{m}$ long device. A reason could be a more profound non-uniformity in channel width and parasitic charges, since at low vertical fields Coulomb scattering is important.

From the slope of the curves depicted in Fig. 2.4, the calculated conduction band offset has been plotted against $W_{\text {FIN }}$ as shown in Fig. 2.5. Values down to $\approx-40 \mathrm{meV}$ are obtained. The error bars in Fig. 2.5 were determined from the error associated with linear fitting of $\ln (\eta)$ vs. $1 / T$ curves. In this figure, a maximum value for the (absolute) band offset has been obtained for a $W_{\text {FIN }}$ of around $12 \mathrm{~nm}$. This can be explained by two 
counteracting mechanisms $[32,34]$. As discussed earlier, when $W_{\text {FIN }}$ is decreasing, the built-in strain due to the TiN layer increases. For all strain conditions this shifts the lowest occupied conduction band, hence the $\Delta_{2}$ valleys, downwards with respect to un-strained condition [37]. However, for $W_{\mathrm{FIN}}<12 \mathrm{~nm}$, quantization of the carriers occurs and this results in an upward shift of the conduction band edge (despite the higher amount of strain). The same trend was obtained by van Hemert et al. [34] in their band offset calculations in which strain and quantization effects were taken into account. Note that the impact of these effects depends on the channel orientation [34], in our case the channel is oriented along [110] direction.

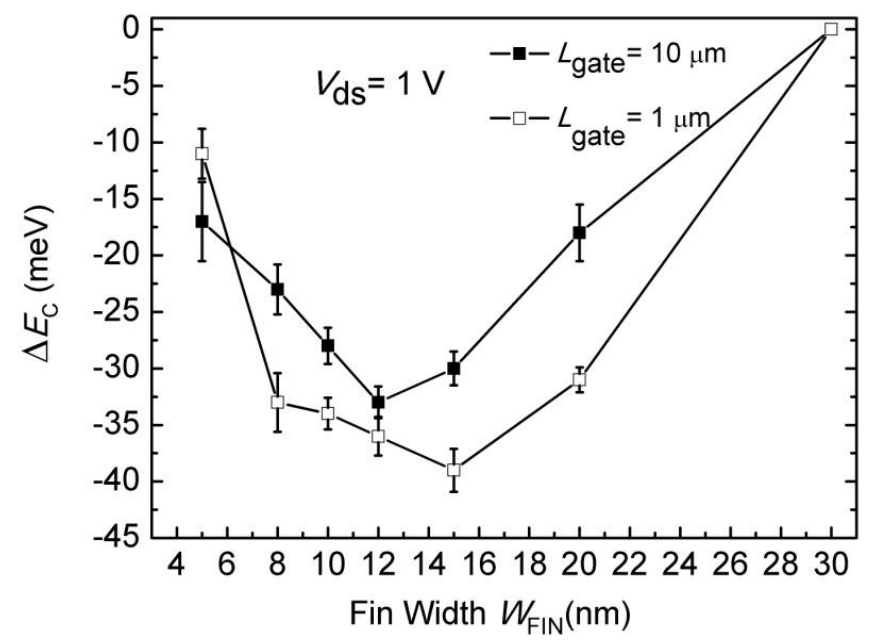

Fig. 2.5 Extracted conduction band offset for a device with a $1 \mu \mathrm{m}$ and $10 \mu \mathrm{m}$ gate length.

\subsubsection{Raman spectroscopy characterization of strain}

A more direct physical characterization technique would complete the strain analysis and could be helpful to support the observations in the electrical data obtained from the NXPTSMC FinFETs. Raman spectroscopy is a non-contact and non-destructive technique that is most widely employed for this purpose [35, 39, 45-48], yielding valuable information about strain even in aggressively scaled device configurations $[40,49]$.

The technique relies on the inelastic scattering, or Raman scattering, of monochromatic light (usually from a laser). The scattering process involves an instantaneous absorption of a photon and subsequent emission of scattered photon. When the emitted photon is elastically scattered, the incident and scattered photon has the same frequency. However, if an inelastic scattering occurs, the frequency of the scattered photon shifts. During inelastic scattering, incident photons excite atom or molecule from the ground to a virtual energy level. While relaxing, it emits a photon and returns to a different rotational or vibrational state. Depending on the energy of the final state with respect to 
initial state, the emitted photons of the laser light will be shifted to higher or lower frequency. This shift provides information about the vibrational, rotational and other transitions. However, inelastic scattering (or Raman scattering) results in a weak signal while the intense part of the signal is due to elastic scattering (or Rayleigh scattering). Therefore, the Rayleigh scattered photons are filtered out to separate the weak Raman scattered photons. Fig. 2.6 illustrates the transitions corresponding to Rayleigh scattering, stokes and anti-Stokes Raman scattering.

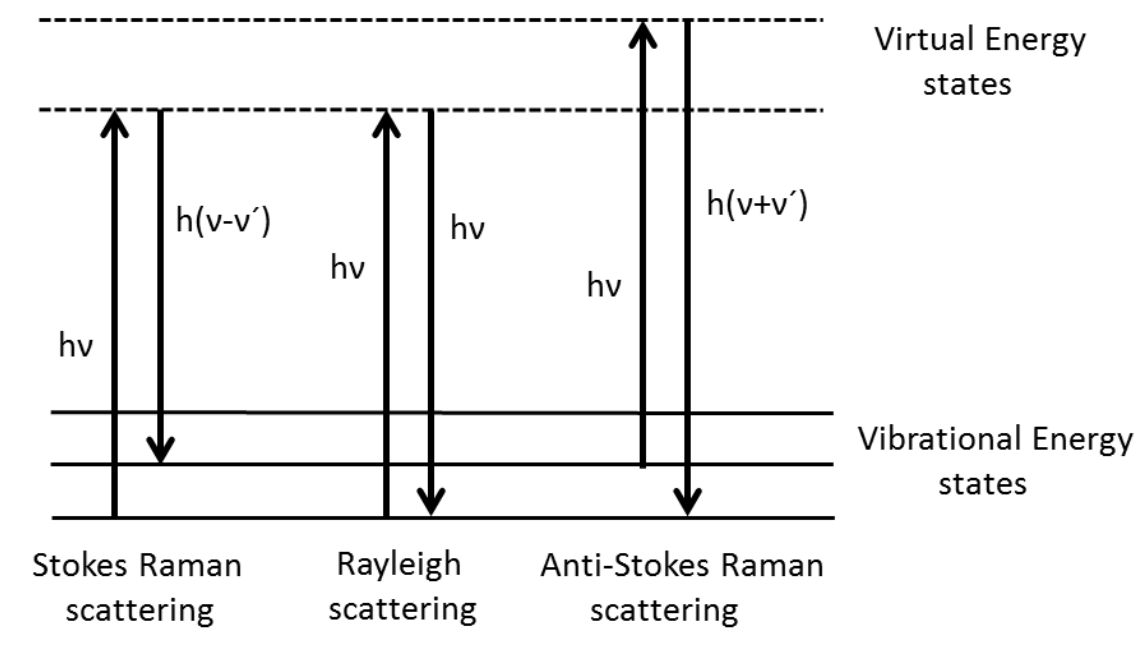

Fig. 2.6 A diagram illustrating the transitions corresponding to Rayleigh scattering, Stokes and antiStokes Raman scattering.

When the crystal lattice is mechanically strained, the atomic distances change. Since the lattice vibration frequency depends on the atomic distance, a vibrational frequency shift occurs. This shift directly affects the Raman peak position. Strain level in the lattice can be determined by using the calibrated Raman peak shift-strain relation.

The Raman spectroscopy measurements have been carried out at room temperature using a confocal Raman microscope (alpha300R, Witech $\mathrm{GmbH}$ ) with $0.8 \mathrm{~cm}^{-1}$ wavenumber resolution (which corresponds to about $0.15 \%$ strain resolution) and a 532 $\mathrm{nm}$ laser source with an output power of $47 \mathrm{~mW}$. This wavelength will give a penetration depth of light in the silicon in the range of $800-1000 \mathrm{~nm}$. This high penetration depth leads to a weak Raman signal from the FinFET with respect to the strong signal from the surrounding relaxed silicon. It is important to note that, since a high penetration depth might be an issue, we performed Raman measurements on the same samples at a shorter wavelength $(454 \mathrm{~nm})$ using the Renishaw Raman tool ${ }^{2}$. In that case, we obtained less significant strain peaks. The wavenumber resolution was relatively low and a lower signal

${ }^{2}$ These measurements were performed with Emile Verstegen (Philips, Eindhoven), with courtesy. 
was obtained from the strained-Si region. The penetration depth of the $454 \mathrm{~nm}$ laser in $\mathrm{Si}$ is around $300 \mathrm{~nm}$, but due to the TiN and poly-Si layers on top of the Si, the signal could not be obtained from the whole strained Si volume. Therefore, although a laser with 532 $\mathrm{nm}$ wavelength had a high penetration depth, we got more signal from the $\mathrm{Si}$ fin compared to a laser with a $454 \mathrm{~nm}$ wavelength. Hence, for our analysis the $532 \mathrm{~nm}$ laser is a better choice.

Metallic tiles were on top of the FinFET structures. The metal layers were very thick and blocking the light. Therefore, they were removed using Focused Ion Beam (FIB) milling. Removal of these layers was necessary to obtain a good Raman signal from the area of interest. Fig. 2.7 shows the HR-SEM picture of a device before and after FIB removal of the metal layers. To prevent the damage to the stressor TiN layer, FIB process parameters were optimized at a $47 \mathrm{pA}$ beam current and a process time of 5 minutes. A cross sectional SEM image after FIB processing confirmed that the stressor layer was still present. Raman spectroscopy on these samples was done by scanning the complete area of the channel region.
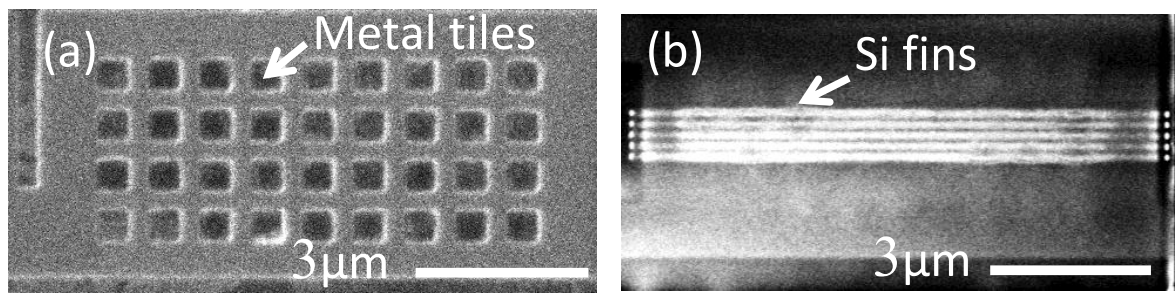

Fig. 2.7 HR-SEM image of a device before (a) and after (b) FIB removal of the metal layers.

Fig. 2.8 shows the peak map of the scanned device region before and after the FIB process. It is clearly observable that the Raman peak of the Si channel region is only detectable after the FIB process. To identify the Si channel from this map, the Raman peak at $520 \mathrm{~cm}^{-1}$ is highlighted. The contrast in this figure indicates different peaks of the Raman spectrum obtained from the channel. This map helps us to identify the Si fin region from the rest of the substrate and it was used for extracting the Raman spectrum of the strained channel region. 

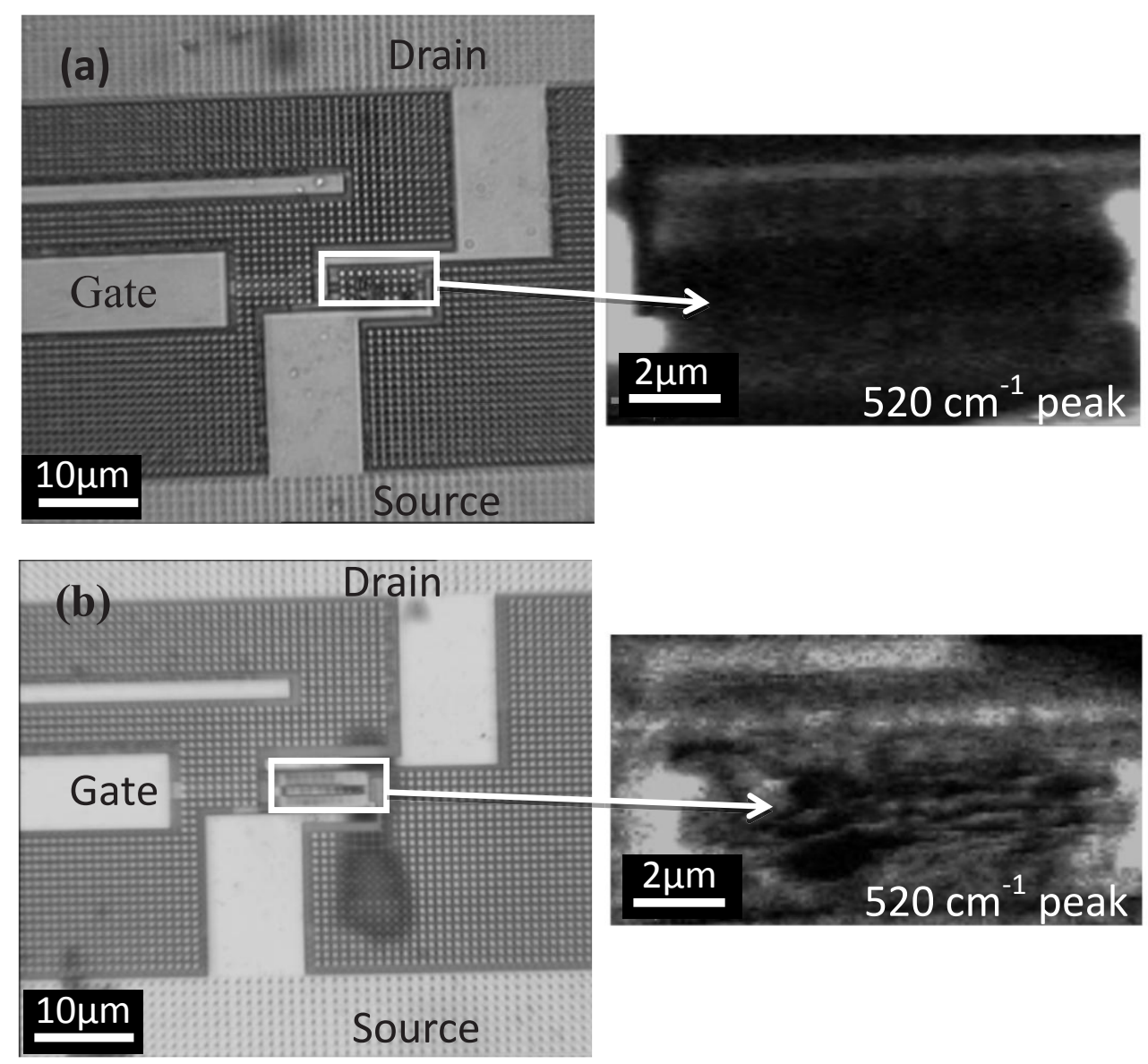

Fig. 2.8 Raman peak map of the channel region (a) before FIB, (b) after FIB preparation. The images on the right side show the peak position map of the Raman spectrum. The $520 \mathrm{~cm}^{-1}$ peak is highlighted with grey tones to identify the Si channel region from the BOX.

The devices with $10 \mu \mathrm{m}$ gate length and different fin widths were used for measurements after FIB preparation. The samples have been analyzed by fitting the obtained Raman peaks with a Lorentzian function. The measurements are shown in Fig. 2.9. 

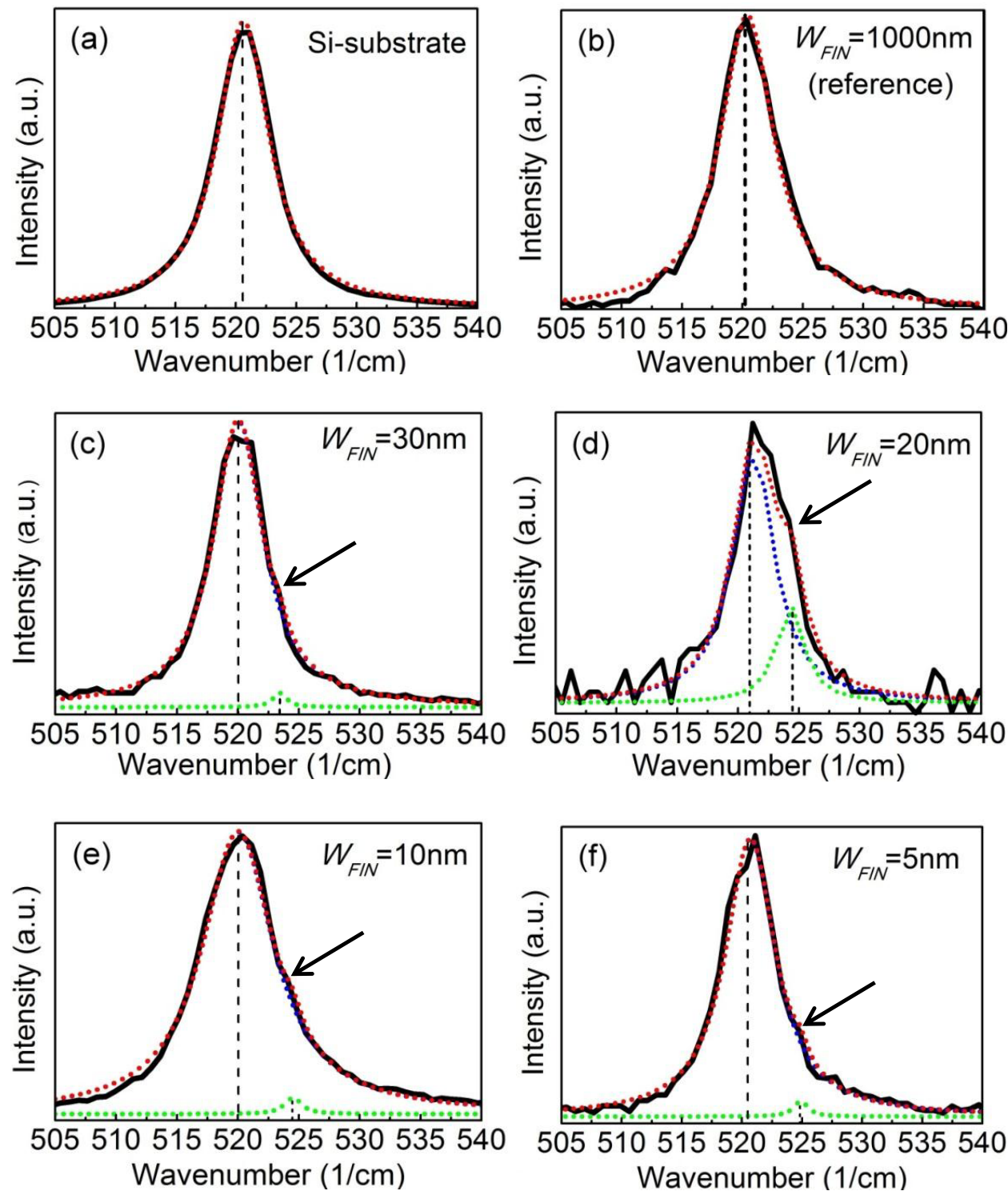

Fig. 2.9 Obtained Raman spectra of (a) the substrate, and that of the FinFET with a fin width of (b) $1000 \mathrm{~nm}$, used as a reference, (c) $30 \mathrm{~nm}$, (d) $20 \mathrm{~nm}$, (e) $10 \mathrm{~nm}$, (f) $5 \mathrm{~nm}$. The blue dotted curves are the obtained Lorentzian fits corresponding to Si substrate. The green dotted curves are after fitting the second peak due to increased strain also indicated by the arrows to guide the eye. The total fits are indicated by red dotted curves. 
In Fig. 2.9 (a) the Si substrate peak is shown. In Fig. 2.9 (b), the peak from the 5-fin FinFET device with $W_{\mathrm{FIN}}=1000 \mathrm{~nm}$ is presented. Since the Si-fin layer is only $60 \mathrm{~nm}$ thick and the penetration depth of the laser is larger than the sum of the Si fin and BOX layer thickness $(\approx 340 \mathrm{~nm})$, the substrate peak as well as the thin un-doped Si peak is present in the Raman spectrum. However, both Raman peaks are from Si and they appear almost at the same wavenumber without stress (i.e. $520 \mathrm{~cm}^{-1}$ ). Therefore, it is not possible to differentiate them. This spectrum is used as a reference for strain calculations. Figs. 2.9 (c)-(f) show a second peak next to the substrate peak in the spectrum. The second peak is from the strained Si fin. It can be seen from Fig. 2.9 that the Si fin peak shifts to higher wavenumbers when the fin width is decreasing from $30 \mathrm{~nm}$ to $5 \mathrm{~nm}$. The shift to higher wavenumbers is an indication of compressive stress [50]. Since the stress is much lower for the $1000 \mathrm{~nm}$ wide fin device, this second, stressed, Si peak cannot be observed. The first peaks are broadened because of the signal from the poly-Si layer on top of the fins [51]. The strained Si peak is weak compared to the substrate peak since the signal from the surroundings is 15-80 times bigger than that of the strained silicon fins. Therefore, for such a sample, it is quite difficult to get a significant signal from strained silicon but could still be used for the analysis.

The strain values were calculated from the Raman peak shift using the $1000 \mathrm{~nm}$ wide fin peak in Fig. 2.9 (b) as a reference. The calculations are dependent on the stress calibration using Raman shift data and the fitting of the peaks. In this way, the strain level and its evolution by fin width is provided. The strain was calculated using the Raman shift according to the following relations [51]:

$$
\begin{gathered}
\sigma=-250(M P a \cdot c m) \cdot \Delta \omega \\
\varepsilon=\frac{\sigma}{E}
\end{gathered}
$$

where $E=$ Young's modulus, $\sigma=$ stress, $\varepsilon=$ strain, $\Delta \omega=$ peak shift.

The Young's modulus of silicon along the $z$ axis is taken as $E_{z}=130 \mathrm{GPa}$ [52] for the strain calculations. Table 2.2 shows the peak positions and calculated strain values for each device. 
Table 2.2: Strain values obtained from a Lorentzian peak fit.

\begin{tabular}{ccc}
\hline \hline Fin width $(\mathrm{nm})$ & Peak Position $\left(\mathrm{cm}^{-1}\right)$ & Strain $(\%)$ \\
\hline \hline 1000 & 520.3 & reference \\
30 & 523.4 & -0.60 \\
20 & 524.2 & -0.75 \\
10 & 524.5 & -0.80 \\
5 & 524.9 & -0.88 \\
\hline \hline
\end{tabular}

Fig. 2.10 shows the extracted strain values using the Raman shift data together with the strain values obtained by N. Serra et al. [37]. The authors used the holographic interferometry method on a similar set of devices to obtain strain information. The strain value obtained for $W_{\mathrm{FIN}}=10 \mathrm{~nm}$ with the Raman spectroscopy method is comparable to Serra's results while for $W_{\mathrm{FIN}}=20 \mathrm{~nm}$ our results indicates a larger strain value. The strain dependency on the fin width is clearly observed in both sets of data. Furthermore, Fig. 2.10 shows that the maximum strain values obtained from FEM simulations (see also section 2.3) agree well with the Raman spectroscopy data for a wide range of fin widths.

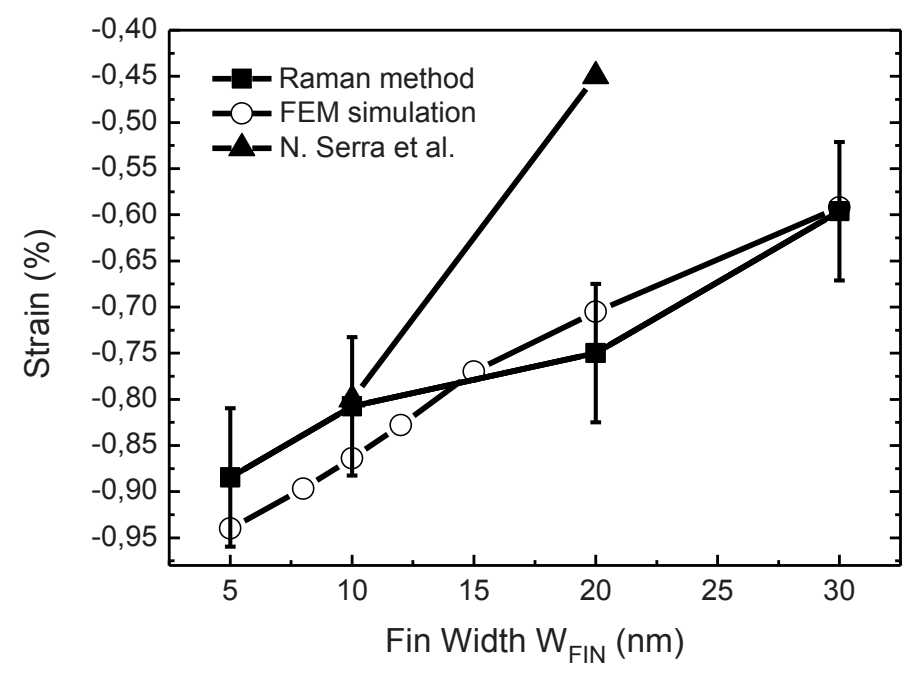

Fig. 2.10 Extracted strain values in silicon FinFETs using Raman spectroscopy. For comparison, FEM simulation and experimental data obtained from the holographic interferometry method [37] have been plotted in the same graph. Note that, due to the limitation of the Raman microscope system, the strain resolution is about $0.15 \%$ for the Raman method. 


\subsubsection{Discussion}

Compressive strain in $\mathrm{Si}$ fin height direction is known to move the $\Delta_{2}$ valleys down, resulting in a decrease in the band gap of (110) oriented Si [9]. However, the band offset has never been experimentally studied in FinFETs. In section 2.3.1.1 the electrical measurements reveal that there is indeed a downward shift of the conduction band edge when the fin width is decreasing down to about $12 \mathrm{~nm}$. Below this value, quantum confinement has a counteractive effect. In addition, the results obtained from optical measurements in section 2.3.1.2 show that strain is increasing for narrow fins. This is in line with the electrical data as well as holographic measurements and FEM simulations. Good agreement in the extracted strain values was obtained from Raman measurements FEM simulations and holographic interferometry. This indicates that Raman spectroscopy is a good method to obtain actual strain values in FinFET structures with nanometer dimensions. Furthermore, results obtained by these analyses can be used to explain the electrical measurements.

\subsection{Conclusions}

In this chapter, a brief introduction to strain, its use and methods in silicon-based devices, and its effect on band gap and mobility in solids is given. To characterize the strain at device level, temperature dependent $I-V$ measurements and Raman spectroscopy technique were utilized for $(1 \overline{1} 0) /[110]$ oriented n-type strained FinFETs. Both techniques provided valuable information about the conduction band offset and strain level in a nanoscale device structure such as the FinFET.

The electrical results indicate that the conduction band energy offset, reaching values down to $\approx-40 \mathrm{meV}$, depends on the dimensions of the fin. This is explained by the superposition of two effects: (1) an increase in compressive strain in the fin height direction for smaller fins moving the $\Delta_{2}$ valleys downward and (2) quantum confinement for fin widths below $12 \mathrm{~nm}$, separating the energy levels available to electrons and moving the conduction band valleys upward.

A relatively small active device area of interest as well as high penetration depth causes a limitation for FinFET type device measurements with Raman spectroscopy. Despite this fact, reasonable results were obtained. It was shown that Raman peaks from Si fins shift to higher wavenumbers for narrow fins indicating compressive strain. Up to $-0.88 \%$ strain has been calculated from our Raman spectroscopy data. FEM simulations and holographic interferometry method were found to point out the same effect which leaded to the results obtained by electrical measurements. These are an indication that Raman 
spectroscopy is an effective method to get strain information, even for small (nanoscale) regions of interest.

\section{References}

[1] D. R. Lovett, "Tensor Properties of Crystals", Adam Hilger, 1989.

[2] J. Bardeen, W. Shockley, "Deformation Potentials and Mobilities in Non-Polar Crystals", Physical Review, 80, pp. 72-80, 1950.

[3] S. C. Smith, "Piezoresistance Effect in Germanium and Silicon", Physical Review, 94, pp. 42-49, 1954.

[4] J. Welser, J. L. Hoyt, J. F. Gibsons, "NMOS and CMOS transistors fabricated in strained silicon/relaxed silicon-germanium structures", Technical Digest International Electron Devices Meeting (IEDM), pp. 1000-1002, 1992.

[5] T. Mizuno, S. Takagi, N. Sugiyama, H. Satake, A. Kurobe, and A. Toriumi, "Electron and hole mobility enhancement in strained-Si MOSFET's on SiGe-on-insulator substrates fabricated by SIMOX technology", IEEE Electron Device Letters, 21, pp. 230-232, 2000.

[6] T. Skotnicki, C. Fenouillet-Beranger, C. Gallon, F. Buf, S. Monfray, F. Payet, A. Pouydebasque, M. Szczap, A. Farcy, F. Arnaud, S. Clerc, M. Sellier, A. Cathignol, J. -P. Schoellkopf, E. Perea, R. Ferrant, and H. Mingam, "Innovative Materials, Devices, and CMOS Technologies for Low-Power Mobile Multimedia", IEEE Transactions on Electron Devices, 55, pp. 96-130, 2008.

[7] F. Rochette, M. Casse, M. Mouis, A. Haziot, T. Pioger, G. Ghibaudo, F. Boulanger, "Piezoresistance effect of strained fully depleted silicon-on-insulator MOSFETs integrating a HfO2/TiN gate stack", Solid-State Electronics, 53, 3, pp. 392-396, 2009.

[8] S. Flachowsky, A. Wei, R. Illgen, T. Herrmann, J. Hontschel, M. Horstmann, W. Klix, and R. Stenzel, "Understanding Strain-Induced Drive-Current Enhancement in Strained-Silicon n-MOSFET and pMOSFET", IEEE Transactions on Electron Devices, 57, pp. 1343-1354, 2010.

[9] N. Serra, and D. Esseni, "Mobility Enhancement in Strained n-FinFETs: Basic Insight and Stress Engineering", IEEE Transactions on Electron Devices, 57, pp. 482-490, 2010.

[10] T. K. Kang, "Evidence for Silicon Band gap Narrowing in Uniaxially Strained MOSFETs Subjected to Tensile and Compressive Stress", IEEE Electron Device Letters, 33, pp. 770-772, 2012.

[11] Y. Sun, S. E. Thompson, T. Nishida, "Strain effect in semiconductors, Theory and device applications", Springer 2010.

[12] S. S. Iyer, P. M. Solomon, V. P. Kesan, A. A. Bright, J. L. Freeouf, T. N. Nguyen, and A. C. Warren, “A gate-quality dielectric system for SiGe metal-oxide-semiconductor devices", IEEE Electron Device Letters, 12, pp. 246-248, 1991.

[13] H.M. Manasevit, I.S. Gergis, A.B. Jones, "Electron mobility enhancement in epitaxial multilayer $\mathrm{Si}_{-} \mathrm{Si}_{1-}$ ${ }_{x} G_{e}$ alloy films on (100) Si", Applied Physics Letters, 41, 5, 1982, pp. 464-466.

[14] J. L. Hoyt, H. M. Nayfeh, S. Eguchi, I. Aberg, G. Xia, T. Drake, E. A. Fitzgerald, and D. A. Antoniadis, "Strained silicon MOSFET technology", Technical Digest International Electron Devices Meeting (IEDM), pp. 23-26, 2002.

[15] W. S. Yoo, T. Ueda, and K. Kang, "Characterization of uni-axially stressed Si and Ge concentration in $\mathrm{Si}_{1-x} \mathrm{Ge}_{\mathrm{x}}$ using polychromator-based multi-wavelength Raman spectroscopy", International Workshop on Junction Technology, pp. 78-83, 2009.

[16] Ji-Song Lim, S.E. Thompson, J.G. Fossum, "Comparison of threshold voltage shifts for uniaxial and biaxial tensile stressed n-MOSFETs", IEEE Electron Device Letters, 25, pp. 731-733, 2004.

[17] T. Ghani, M. Armstrong, C. Auth, M. Bost, P. Charvat, G. Glass, T. Hoffmann, K. Johnson, C. Kenyon, J. Klaus, B. McIntyre, K. Mistry, A. Murthy, J. Sandford, M. Silberstein, S. Sivakumar, P. Smith, K. Zawadzki, S. Thompson, and M. Bohr, "A 90nm high volume manufacturing logic technology 
featuring novel $45 \mathrm{~nm}$ gate length strained silicon CMOS transistors", Technical Digest International Electron Devices Meeting (IEDM), pp. 978-980, 2003.

[18] S. E. Thompson, G. Sun, Y. S. Choi, and T. Nishida, "Uniaxial-process-induced strained-Si: extending the CMOS roadmap", IEEE Transactions on Electron Devices, 53, pp. 1010-1020, 2006.

[19] K. Shin, C. O. Chui, T. J. King, "Dual stress capping layer enhancement study for hybrid orientation FinFET CMOS technology", Technical Digest International Electron Devices Meeting (IEDM), pp. 988991, 2005.

[20] S. L. Wu, Y. M. Lin, S. J. Chang, S. C. Lu, P. S. Chen, and C. W. Lui, "Enhanced CMOS performances using substrate strained-SiGe and mechanical strained-Si technology", IEEE Electron Device Letters, 27, pp. 46-48, 2006.

[21] N. Collaert, A. de Keersgieter, K. G. Anil, R. Royackers, G. Eneman, M. Goodwin, B. Eyckens, E. Sleeckx, J. F. de Marneffe, K. de Mayer, P. Absil, M. Jurczak, and S. Biesemans, "Performance improvement of tall triple gate devices with strained SiN layers", IEEE Electron Device Letters, 26, pp. 820-822, 2006.

[22] K. Rim, R. Anderson, D. Boyd, F. Cardone, K. Chan, H. Chen, S. Christansen, J. Chu, K. Jenkins, T. Kanarsky, S. Koester, B. H. Lee, K. Lee, V. Mazzeo, A. Mocuta, D. Mocuta, P. M. Mooney, P. Oldiges, J. Ott, P. Ronsheim, R. Roy, A. Steegen, M. Yang, H. Zhu, M. leong, and H. S. P. Wong, "Strained Si CMOS (SS CMOS) technology: Opportunities and challenges", Solid state electronics, 42, pp.11331139, 2003.

[23] F. Conzatti, N. Serra, D. Esseni, M. De Michielis, A. Paussa, P. Palestri, L. Selmi, S. M. Thomas, T. E. Whall, D. Leadley, E. H. C. Parker, L. Witters, M. J. Hytch, E. Snoeck, T. J. Wang, W. C. Lee, G. Doornbos, G. Vellianitis, M. J. H. van Dal, and R. J. P. Lander, "Investigation of Strain Engineering in FinfETs Comprising Experimental Analysis and Numerical Simulations", IEEE Transactions on Electron Devices, 58, pp. 1583-1593, 2011.

[24] K. Rim, S. Koester, M. Hargrove, J. Chu, K.A Jenkins, P. Mooney, J. Ott, T. Kanarsky, P. Ronsheim, M. leong, and H. S. Wong, "Strained Si nMOSFETs for high performance CMOS technology", IEEE Symposium on Technology, Digest of Technical Papers, pp. 59-60, 2001.

[25] S. Mayuzumi, S. Yamakawa, D. Kosemura, M. Takei, K. Nagata, H. Akamatsu, H. Wakabayashi, K. Amari, Y. Tateshita, M. Tsukamoto, T. Ohno, A. Ogura and N. Nagashima, "Mobility and Velocity Enhancement Effects of High Uniaxial Stress on Si (100) and (110) Substrates for Short-Channel pFETs", IEEE Transactions on Electron Devices, 57, pp. 1295 -1300, 2010.

[26] C. Y. Kang, R. Choi, M. M. Hussain, J. Wang, Y.J. Suh, H. C. Floresca, M. J. Kim, J. Kim, B. H. Lee, R. Jammy, "Effects of metal gate-induced strain on the performance of metal-oxide-semiconductor field effect transistors with titanium nitride gate electrode and hafnium oxide dielectric", Applied Physics Letters, 91, pp. $033511-033511-3,2007$.

[27] B. Delley, and E. Steigmeier, "Size dependence of band gaps in silicon nanostructures", Applied Physics Letters, 67, pp. 2370-2372, 1995.

[28] Z. Lu, and D. Grozea, "Crystalline Si/SiO2 quantum wells," Appl. Phys. Lett., 80, pp. 255-257, 2002.

[29] L. Ge, and J. Fossum, "Analytical modeling of quantization and volume inversion in thin Si-film DG MOSFETs," IEEE Trans. Electron Devices, 49, pp. 287-294, 2002.

[30] K. Rim, J. Chu, K.A Jenkins, T. Kanarsky, K. Lee, A. Mocuta, H. Zhu, R. Roy, J. Newbury, J. Ott, K. Petrarca, P. Mooney, D. Lacey, S. Koester, K. Chan, D. Boyd, M. leong, and H. S. Wong, "Characteristics and device design of sub-100 nm strained Si $n$ and pMOSFET", IEEE Symposium on VLSI Technology, Digest of Technical Papers, pp. 98-99, 2002.

[31] S. F. Feste, J. Knoch, S. Habicht, D. Buca, Q. T. Zhao, S. Mantl, "Silicon nanowire FETs with uniaxial tensile strain", Solid State Electronics, 53, pp. 1257-1262, 2009.

[32] T. van Hemert, B. Kaleli Kemaneci, R.J.E. Hueting, D. Esseni, M.J.H. van Dal, and J. Schmitz, "Extracting the conduction band offset in strained FinFETs by subthreshold measurements", Proceedings of the European Solid-State Device Research Conference (ESSDERC), pp. 275-278, 2011. 
[33] B. Kaleli Kemaneci, T. van Hemert, R.J.E. Hueting, and R.A.M. Wolters, "Strain characterization of FinFETs using Raman spectroscopy", Presented at the European Materials Research Symposium (EMRS), 2012.

[34] T. van Hemert, B. Kaleli Kemaneci, R.J.E. Hueting, D. Esseni, M.J.H. van Dal, and J. Schmitz, "Strain and Conduction Band Offset in narrow n-type FinFETs", IEEE Transactions on Electron Devices, 60, pp. 1005-1010, 2013.

[35] B. Kaleli, T. van Hemert, R.J.E. Hueting, and R.A.M. Wolters, "Strain characterization of FinFETs using Raman spectroscopy", Thin-Solid Films, 541, pp. 57-61, 2013.

[36] J. G. Wang, Jiyoung Kim, Chang Yong Kang, Byoung Hun Lee, Raj Jammy, Rino Choi, and M. J. Kim, "Origin of tensile stress in the Si substrate induced by TiN/HfO2 metal gate/high-k dielectric gate stack", Applied Physics Letters, 93, pp. 161913 -161913-3, 2008.

[37] N. Serra, F. Conzatti, D. Esseni, M. De Michielis, P. Palestri, L. Selmi, S. Thomas, T. E. Whall, E. H. C. Parker, D. R. Leadley, L. Witters, A. Hikavyy, M. J. Hytch, F. Houdellier, E. Snoeck, T. J. Wang, W. C. Lee, G. Vellianitis, M. J. H. van Dal, B. Duriez, G. Doornbos, and R. J. P. Lander, "Experimental and physics-based modeling assessment of strain induced mobility enhancement in FinFETs", Technical Digest International Electron Devices Meeting (IEDM), pp. 1-4, 2009.

[38] M. van Dal, N. Collaert, G. Doombos, G. Vellianitis, G. Curatola, B. Pawlak, R. Duffy, C. Jonville, B. Degroote, E. Altamirano, E. Kunnen, M. Demand, S. Beckx, T. Vandeweyer, C. Delvaux, F. Leys, A. Hikavyy, R. Rooyackers, M. Kaiser, R. Weemaes, S. Biesemans, M. Jurczak, K. Anil, L. Witters, R. Lander, "Highly manufacturable FinFETs with sub-10nm fin width and high aspect ratio fabricated with immersion lithography", IEEE Symposium on VLSI Technology, Digest of Technical papers, pp. 110-111, 2007.

[39] I. de Wolf, "Micro-Raman spectroscopy to study local mechanical stress in silicon integrated circuits", Semiconductor Science and Technology, 11, pp. 139-154, 1996.

[40] K. E. Moselund, P. Dobrosz, S. Olsen, V. Pott, L. De Michielis, D. Tsamadoa, D. Bouvet, A. O'Neill, and A. M. Ionescu, "Bended Gate-All-Around Nanowire MOSFET: a device with enhanced carrier mobility due to oxidation-induced tensile stress", Technical Digest International Electron Devices Meeting (IEDM), pp. 191-194, 2007.

[41] T. K. S. Wong, Y. Gong, P. Yang, and C. M. Ng, "Characterization of biaxial stressed silicon by spectroscopic ellipsometry and synchrotron x-ray scattering", Semiconductor Science and Technology, 22, pp. 1232-1239, 2007.

[42] M. Belyansky, N. Domenicucci, J. Li Klymko, and A. Madan, "Strain characterization: techniques and applications", Solid State Technology, 52, 2, pp. 26-29, 2009.

[43] J. L.-P. J. van der Steen, R. J. E. Hueting, and J. Schmitz, "Extracting energy band offsets on thin silicon-on-insulator MOSFETs", Proceedings of the European Solid-State Device Research Conference (ESSDERC), pp. 242-245, 2008.

[44] F. Gamiz and A. Godoy, "FinFETs and other multi gate transistors, Chapter 5: Mobility in Multigate MOSFETs", Edited by J.-P. Colinge, Springer, 2008.

[45] E. Anastassakis, A. Pinczuk, E. Burstein, F. H. Pollak and M. Cardona, "Effect of static uniaxial stress on the Raman spectrum of Silicon", Solid State Communications, 8, pp. 133-138, 1970.

[46] A. Ogura, D. Kosemura, M. Takei, H. Uchida, N. Hattori, M. Yoshimaru, S. Mayuzumi, and H. Wakabayashi, "Evaluation of local strain in Si using UV-Raman spectroscopy", Materials Science and Engineering B, 159, pp. 206-211, 2009.

[47] S. Nishibe, T. Sasaki, H. Harima, K. Kisoda, T.Yamazaki, and W.S. Yoo, "Raman study on the process of Si advanced integrated circuits", 14th IEEE International Conference on Advanced Thermal Processing of Semiconductors, pp. 211-215, 2006.

[48] P. Moens, J. Roig, F. Clemente, I. De Wolp, B. Desoete, F. Bauwens, and M. Tack, "Stress-Induced Mobility Enhancement for Integrated Power Transistors", Technical Digest International Electron Devices Meeting (IEDM), pp. 877-880, 2007. 


\section{Chapter 2}

[49] W. Xiong, C. R. Cleavelin, P. Kohli, C. Huffman, T. Schulz, K. Schruefer, G. Gebara, K. Mathews, P. Patruno, Y. M. Le Vaillant, I. Cayrefourcq, M. Kennard, C. Mazure, K. Shin, and T. J. K. Liu, "Impact of strained-silicon-on-insulator (sSOI) substrate on FinFET mobility", IEEE Electron Device Letters, 27, pp. 612-614, 2006.

[50] W. S. Yoo, T. Ueda, K. Kang, "Characterization of uni-axially stressed $\mathrm{Si}$ and Ge concentration in $\mathrm{Si}_{1}$ ${ }_{x} \mathrm{Ge}_{\mathrm{x}}$ using polychromator-based multi-wavelength Raman spectroscopy", International Workshop on Junction Technology, pp. 78-83, 2009.

[51] V. Paillard, P. Puech, M. A. Laguna, P. Temple-Boyer, and B. Caussat, "Resonant Raman scattering in polycrystalline silicon thin films", Applied Physics Letters, 73, pp. 1718-1720, 1998.

[52] M. A. Hopcroft, W. D. Nix, and T. W. Kenny, "What is the Young's Modulus of Silicon?", Journal of Microelectromechanical Systems, 19, pp. 229-238, 2010. 


\section{Ferroelectric thin-film PZT layers}

This chapter starts with a brief introduction to ferroelectrics and their applications. Then, the characterization of thin-film PZT/LNO stacks on an encapsulated TiN electrode is described for different stack layer thicknesses. Analysis of the electrical measurements indicates ferroelectric and piezoelectric properties and that a non-ferroelectric layer appears to be present at the PZT-electrode interface. The presented results in this chapter are important for the piezoelectric field-effect transistor (Chapter 5), in which these thin-film PZT/LNO layers have been integrated. 


\subsection{Introduction}

Ferroelectricity is a property of a material that can reverse the spontaneous polarization by the application of an external electric field [1]. Ferroelectric materials also show piezoelectric properties. These materials generate a voltage when deformed by a mechanical force (i.e. piezoelectric effect) or physically changes in shape when a voltage is applied (i.e. converse piezoelectric effect).

Ferroelectric films attract much interest due to their applications in high density capacitors [2], sensors, micromechanical systems [3, 4] and nonvolatile memories [5, 6]. Recently, there have been a growing number of investigations on novel steepsubthreshold devices where thin ferroelectric or piezoelectric layers are employed. Salahuddin et al. [7] came up with a concept of using ferroelectrics as a gate dielectric to obtain a negative capacitance and hence improvement of the device properties. Later on, Rusu et al. [8] have demonstrated this concept experimentally on p-MOSFET devices. Their results show that it is possible to obtain a device with sub-threshold swing below the fundamental limit of $60 \mathrm{mV} / \mathrm{dec}$ at room temperature [9]. Newns et al. [10] proposed the piezoelectric transistor (PET) based on piezo-resistance effect on special channel materials manifesting a pressure-induced metal-insulator transition. They reported that the performance of PET's outpaces the conventional FETs with respect to the lower supply voltage and higher switching speed.

Another way to make use of the converse piezoelectric effect is to employ piezoelectric materials as a channel-stressor in a field-effect transistor. The application of stressors in $\mathrm{Si}$ technology was discussed in Chapter 2. So far, a piezoelectric stressor in a device structure has been proposed but not realized [11]. Simulations show that, if a proper strain configuration is formed, the sub-threshold swing (SS) can be decreased by $\approx 10 \%$. The strain distribution differs depending on the channel dimensions and the integrated materials. As demonstrated in Chapter 2, a large amount of strain can be obtained in narrow channels. This in combination with their strongly reduced short-channel effects makes FinFETs potentially attractive devices for integrating piezoelectric FETs (PiezoFETs). Lead zirconate titanate or PZT $\left(\mathrm{Pb}\left[\mathrm{Zr}_{\mathrm{x}} \mathrm{Ti}_{1-\mathrm{x}}\right] \mathrm{O}_{3}\right)$ is an attractive material for these applications, because of its relatively high piezoelectric response compared to other materials. PZT is a ceramic perovskite material and can be grown with different $\mathrm{Zr}$ to $\mathrm{Ti}$ concentration ratio that can change the properties. The PZT systems with a composition close to the morphotropic phase boundary (MPB, $\mathrm{Ti} / \mathrm{Zr}=48 / 52$ ) have high dielectric permittivity and piezoelectric response $[12,13]$. The strong piezoelectric effect of these systems is attributed to the increased number of allowable domain states at the MPB [14]. Aluminum-Nitride (AIN) can be an alternative piezoelectric material to PZT, with 
better CMOS compatibility and, however, a lower piezoelectric constant. It is also used in this work (see chapter 5 ).

The properties of PZT films are strongly dependent on the surrounding layers, the dimensions, and the fabrication conditions. Since the film/electrode interface becomes more prominent with scaling, thickness reduction may lead to reduced piezoelectric performance. In earlier works on scaling of PZT, small substrates and a buffer/electrode layer thicker than one hundred nanometer were used $[15,16]$. These buffer/electrode layers were generally grown to relatively thick dimensions to keep control of the PZT growth orientation and to avoid ferroelectric performance degradation [17-19]. However, very thick PZT stacks are ineffective stressors due to the clamping effect [20].

The integration of ferroelectric/piezoelectric action in device structures could enable new functionalities. However, thick layer stacks do not meet the requirements for low-power, low voltage applications and are not compatible with the emerging technology $[7,10,11$, 15]. Uniform very thin films on large substrates are desired for the integration with nanoscaled devices in Si technology [8]. Therefore, there is a need for further research.

With this motivation, this chapter presents the analysis of thin-film $\mathrm{PbZr}_{0.52} \mathrm{Ti}_{0.48} \mathrm{O}_{3} / \mathrm{LaNiO}_{3}$ (LNO) stacks on the commonly used poly-Si/TiN gate stack for CMOS technology. LNO was used as a buffer layer and a diffusion barrier. TiN is a widely used material in advanced IC technology, due to the high temperature stability, mid-gap work function, low resistivity and good barrier properties [21]. The use of this material as a bottom electrode in PZT structures enables application of ferroelectrics in future Si technology nodes, such as a stressor for FETs [11]. Experimental results of the piezo-FinFET realized in the MESA+ Nanolab are presented in chapter 5.

\subsection{PZT capacitors}

\subsubsection{Capacitor fabrication}

Capacitor structures were realized on $100 \mathrm{~mm}$ Si wafers to characterize the thin PZT/LNO stacks. A schematic drawing of these structures is shown in Fig. 3.1. The processing started with a $5 \mathrm{~nm}$ TiN layer deposition on $\mathrm{SiO}_{2}$ by atomic layer deposition (ALD). Subsequently, a $10 \mathrm{~nm}$ amorphous-Si layer was deposited without vacuum break by chemical vapor deposition (CVD) to prevent oxidation of TiN. This encapsulated TiN, a widely employed gate metal in advanced Si technology [21], was used as a bottom electrode for the PZT thin film capacitors. Then, the wafers were annealed at $900{ }^{\circ} \mathrm{C}$ for 30 seconds by rapid thermal annealing. As a result, the amorphous-Si layer is crystallized. As a buffer layer, LNO films of $5 \mathrm{~nm}, 10 \mathrm{~nm}$ and $20 \mathrm{~nm}$ thicknesses and homogeneous PZT films of $75 \mathrm{~nm}$ and $100 \mathrm{~nm}$ thicknesses were grown at $650{ }^{\circ} \mathrm{C}$ using a SolMateS large-area pulsed laser deposition (LAPLD) system. More information about the deposition process 
can be found in ref. [22]. We kept the LNO buffer layer as thin as possible for the envisaged PZT properties. Finally, the PZT as well as the LNO film were patterned by wet etching by a 2 step process. Firstly, $5 \mathrm{sec}$ in BHF (12.5 \%): $\mathrm{HNO}_{3}: \mathrm{NH}_{4} \mathrm{Cl}: \mathrm{H}_{2} \mathrm{O}$ (8:1:8:32) at room temperature and secondly, $5 \mathrm{sec}$ in $\mathrm{HCl}(45 \%): \mathrm{H}_{2} \mathrm{O}(1: 1)$ at $45^{\circ} \mathrm{C}$. This 2-step process was repeated until the layer was completely etched. The first solution is an etching solution while the second one serves as a surface cleaner. After etching, a top electrode stack of $10 \mathrm{~nm} \mathrm{Ti}$ and $100 \mathrm{~nm}$ Pt was sputtered through a shadow mask to fabricate $200 \times$ $200 \mu \mathrm{m}^{2}$ capacitors [23]. Note that the resistivity of the encapsulated TiN layer was measured to be $\approx 160 \mu \Omega$-cm at room temperature (see chapter 4 ).

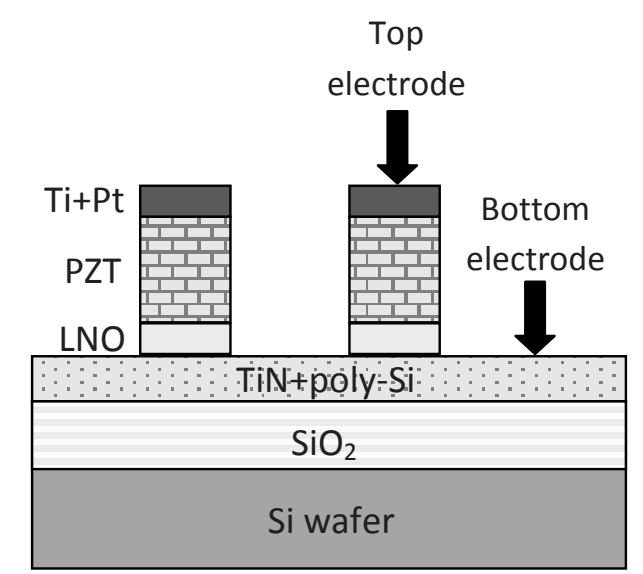

Fig. 3.1 Schematic drawing of the PZT capacitors. The dimensions are not to scale.

\subsubsection{Characterization of the PZT stacks}

\subsubsection{XPS depth profile}

X-ray photoelectron spectroscopy (XPS) has been used to obtain the compositional depth profile of the fabricated multi-film stack. XPS analysis is important to get information on the material distribution in different layers and on interfaces. It can detect diffusion of impurity atoms (e.g. Pb) into the silicon below the PZT/LNO stack. Impurity diffusion can degrade the performance of the underlying transistor.

Fig. 3.2 (a) shows the XPS depth profile in a capacitor. Starting from the surface, metallic layers of Pt and Ti are recognized in the XPS depth profile. Then, after about 6 minutes of sputtering, the PZT layer is reached. Below that layer the LNO, poly-Si and TiN layers can be observed. Fig. 3.2 (b) shows a magnification of the $\mathrm{PZT}-\mathrm{Si}-\mathrm{TiN}-\mathrm{SiO}_{2}$ interface region. No diffusion of $\mathrm{Pb}$ in the $\mathrm{Si}$ layer is observed within the resolution limit of XPS $(\approx 0.5$ at. \%). This implies that, when deposited on FinFETs, no degradation of the transistor properties is expected. 

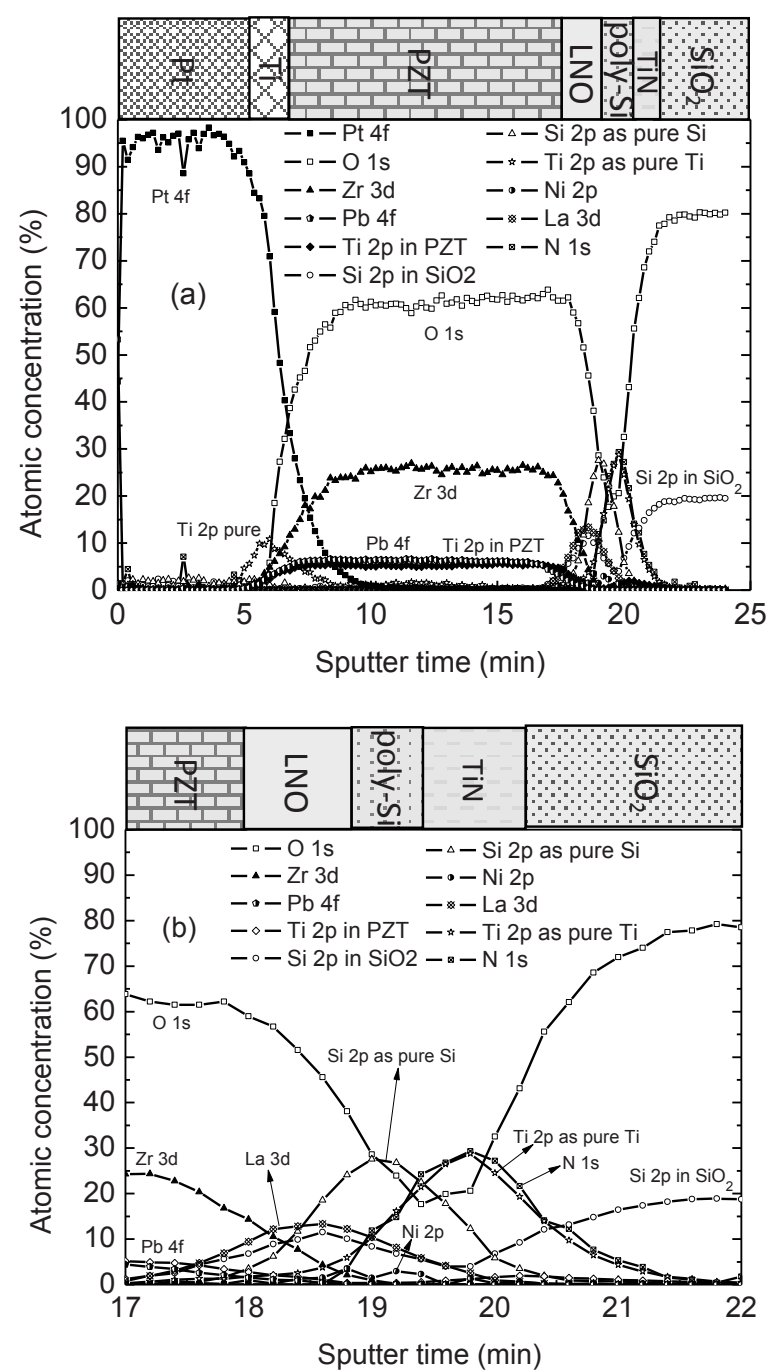

Fig. 3.2 XPS depth profile of the PZT capacitor (a) from top to $\mathrm{SiO}_{2}$ layer, (b) magnified PZT-LNOpoly-Si-TiN-SiO ${ }_{2}$ stack. The layer stack is schematically drawn on top of the graphs to guide the eye.

\subsubsection{XRD analysis}

Ferroelectric film properties are both affected by orientation and crystallinity. X-ray diffraction (XRD) analysis is typically used for analyzing the crystalline properties of the material/film. For instance, Tsukada et al. [24] demonstrated that if the volume fraction of $(001)$ orientation in the $(001) /(100)$-oriented films is high the switchable polarization will also be high. Since the polarization direction is easily aligned to the c-axis (001) in tetragonal PZT, this orientation could give higher polarization. Furthermore, Nagashima et al. [25] reported that the $\mathrm{Zr} / \mathrm{Ti}$ ratio influences the crystal orientation dependence of the ferroelectric properties. 
The XRD spectra of the $100 \mathrm{~nm}$ and $75 \mathrm{~nm}$ thick PZT films on different LNO thicknesses are shown in Fig. 3.3 (a) and (b). These spectra show a typical perovskite polycrystalline PZT structure without preferred orientation. Except for $5 \mathrm{~nm} \mathrm{LNO}$, both $(100) /(001)$ and minor $(110) /(101)$ orientations are observed. However, a $60 \mathrm{~nm}$ PZT film showed only a weak (100)/(001) XRD peak (data not shown). Since our samples show major (100)/(001) orientations, we expect that these films have good ferroelectric properties.
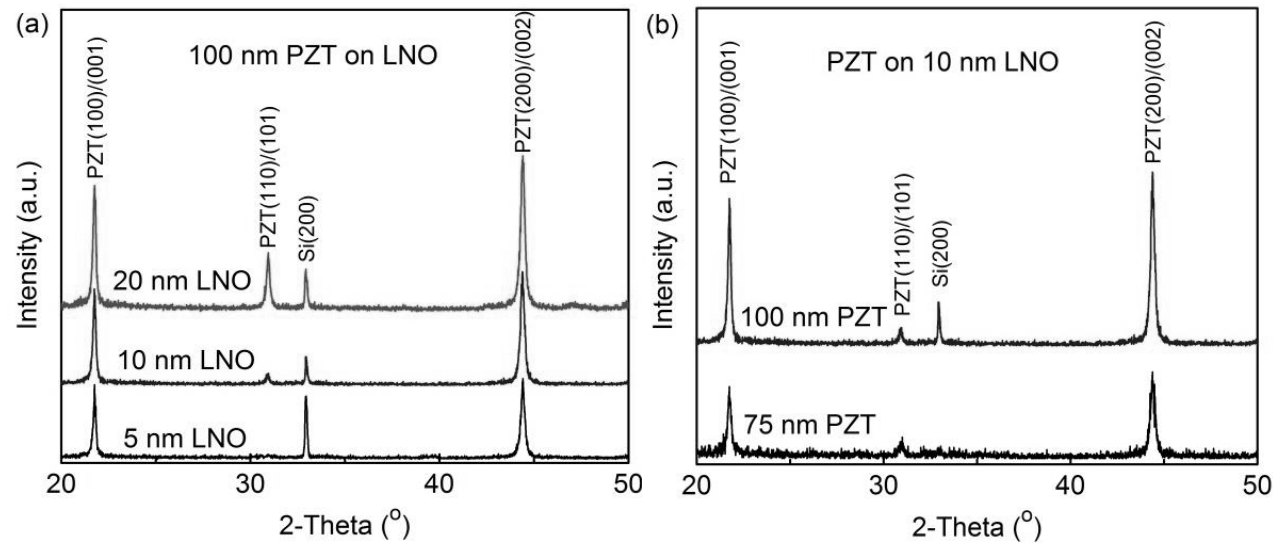

Fig. 3.3 XRD graphs of (a) a 100 nm-thick PZT film on different LNO layer thicknesses and (b) a 75 $\mathrm{nm}$ and a $100 \mathrm{~nm}$ PZT film on a $10 \mathrm{~nm}$-thick LNO layer.

\subsubsection{Laser Doppler vibrometer measurements}

A Polytec MSA-400 Micro-scanning laser Doppler vibrometer (LDV) tool was used in this study. The LDV method is utilized for non-contact measurement of surface vibrations [26, 27]. A schematic set-up of an LDV system is shown in Fig. 3.4. The laser beam reaching the surface is reflected by the sample and detected by a measurement system. From the Doppler shift of the laser beam frequency, the amplitude and frequency of the surface vibrations can be obtained. 


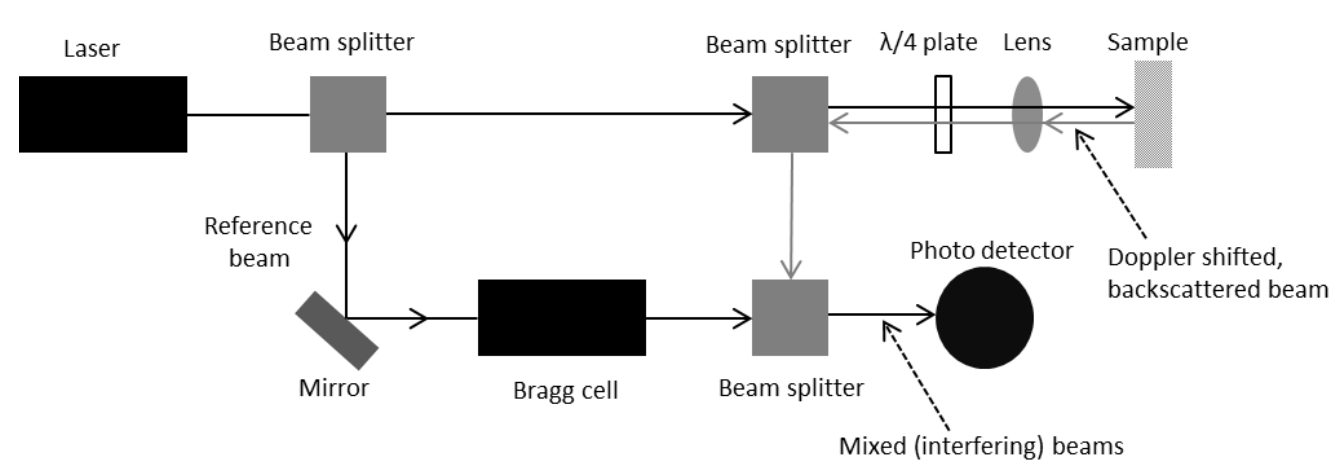

Fig. 3.4 Schematic set-up of the LDV system

In the measurement system, the He-Ne laser $(\lambda=632.8 \mathrm{~nm})$ beam is split by the first beam splitter into a reference beam and a measurement beam. The measurement beam passes through a second beam splitter and focuses on the object or sample. The object reflects the beam and the beam deflects towards a second beam splitter. In the meantime, the reference beam passes through the Bragg cell, which gives a frequency shift. The measurement beam and the reference beam are merged by a third beam splitter and then directed onto a photo detector. The superposition of the reference and the measurement beam generates an interference pattern. As the path length of the beam is constant over time, dark and bright fringes are generated. One complete dark-bright cycle corresponds to the displacement of a half of the laser wavelength (in case of the $\mathrm{He}-\mathrm{Ne}$ laser, this is around $316 \mathrm{~nm}$ ). The change in the path length per unit time corresponds to a Doppler shift of the beam. The velocity of the object can be directly determined by the modulation frequency of the interferometry pattern. If the sample generates the same pattern while moving forward and backward, the direction of the pattern cannot be determined without the help of a Bragg cell. The Bragg cell shifts the light frequency by $40 \mathrm{MHz}$ and enables detecting upward and backward motion [28].

In this study, the effective piezoelectric coefficient $\left(d_{33, f}\right)$ was determined using the laser Doppler vibrometer (LDV) method with a resolution in the pm range [28]. The piezoelectric capacitor was excited by applying a $1 \mathrm{~V}$ dc-voltage and a sinusoidal acvoltage of $2 \mathrm{~V}$ at $8 \mathrm{kHz}$ frequency at the top electrode. The response under these measurement conditions is shown in Fig. 3.5 (a) for an upward displacement. From the line scan profile in Fig. 3.5 (b) a maximum $d_{33, f}$ coefficient is calculated as $\delta_{\text {top }} / V_{\text {ac }}=53$ $\mathrm{pm} / \mathrm{V}$. This value is very close to reported values obtained from relatively thick PZT layers $(800 \mathrm{~nm})$ [29]. When the PZT film is used for steep sub-threshold devices, it is crucial to have films with a high $d_{33, f}$ value [11]. Similar $d_{33, f}$ values were obtained for a $75 \mathrm{~nm}$ thick PZT film. 

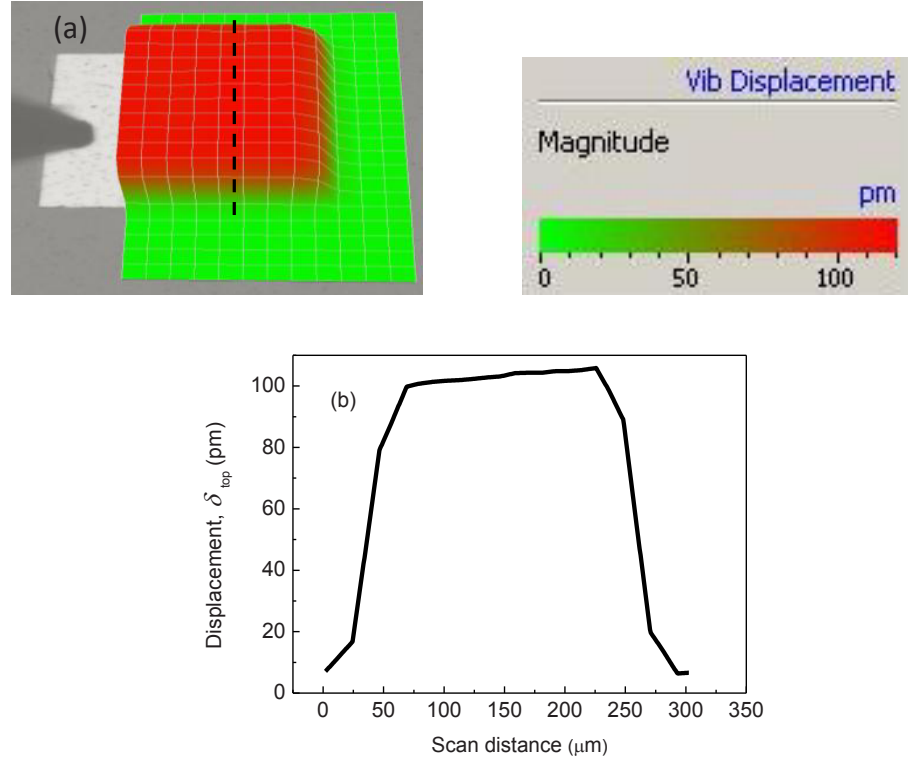

Fig. 3.5 (a) 3D upward response scan of the two dimensional piezoelectric layer. A $1 \mathrm{~V}$ dc and a $2 \mathrm{~V}$ ac voltage at $8 \mathrm{kHz}$ frequency was applied to the film. (b) A line scan profile of the piezoelectric displacement of top electrode across the dashed line. The piezoelectric coefficient is $d_{33, f}=53$ $\mathrm{pm} / \mathrm{V}$. Note that a $20 \mathrm{~nm}$ LNO $+100 \mathrm{~nm}$ PZT stack was used in this experiment.

\subsubsection{Electrical and ferroelectric characterization}

\subsubsection{Polarization hysteresis measurements}

The polarization hysteresis loop measurement is an indicator of the ferroelectric behavior of the material. The measurements in this work were carried out by using an aixACCT Analyzer TF $2000^{1}$. The polarization hysteresis $(P-E)$ loops of the PZT capacitors for an LNO layer thickness of $10 \mathrm{~nm}$ and $20 \mathrm{~nm}$ are shown in Fig. 3.6. No change in remnant polarization $\left(P_{r}\right)$ is observed when the LNO thickness is decreased from $20 \mathrm{~nm}$ to $10 \mathrm{~nm}$. However, for an LNO thickness of $5 \mathrm{~nm}$, the $P_{r}$ drops abruptly (data not shown). Therefore, in this study, the LNO layer thickness was kept at the minimum of $10 \mathrm{~nm}$ for all PZT samples. For the $100 \mathrm{~nm}$ and $75 \mathrm{~nm}$ thick PZT layers, average $P_{r}$ values of $19 \mu \mathrm{C} / \mathrm{cm}^{2}$ and $18 \mu \mathrm{C} / \mathrm{cm}^{2}$ are obtained, respectively. The average coercive field is approximately 200 $\mathrm{kV} / \mathrm{cm}$. The $P$-E loops show offsets both in the field and in the polarization axis. A possible explanation will be discussed in the next section.

\footnotetext{
${ }^{1}$ The measurements were performed at the Inorganic Materials Science group (MESA+ Research Institute for Nanotechnology, University of Twente, The Netherlands.) 


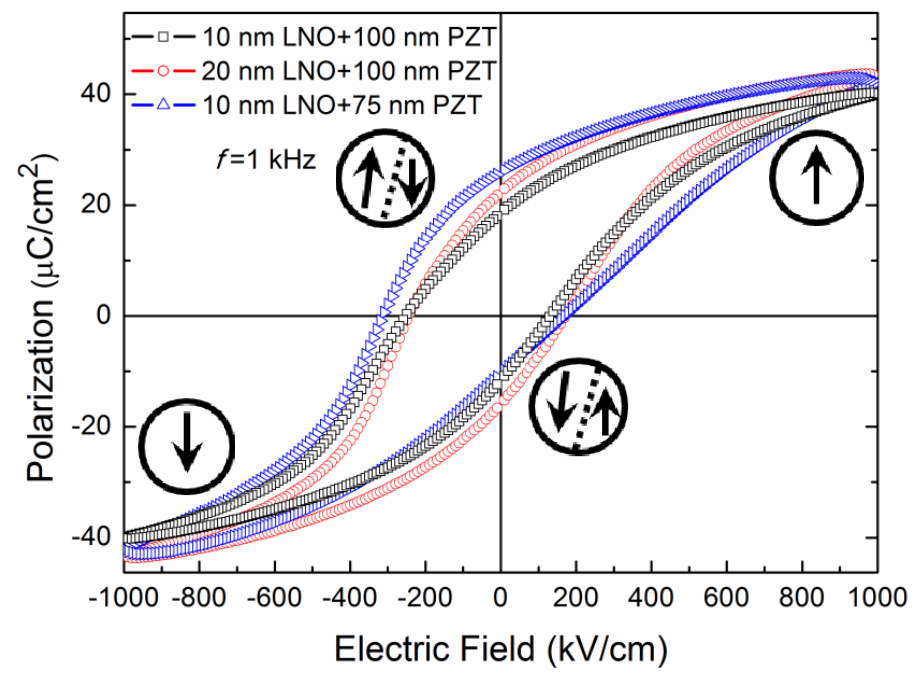

Fig. 3.6 Thickness dependence of polarization hysteresis (P-E) loops. The P-E loops were performed at an applied ac-electric field of $\pm 1000 \mathrm{kV} / \mathrm{cm}$ and $1 \mathrm{kHz}$ frequency. The arrows indicate the polarization state of the material at the indicated fields. The bottom electrode was grounded and a maximum dc bias of $10 \mathrm{~V}$ was applied to the top electrode (see Fig. 3.1).

\subsubsection{C-E and J-E measurements}

The electrical performance of the PZT layer in terms of leakage current and capacitance is also of major importance for device applications. Capacitance-electric field $(C-E)$ and temperature dependent leakage current density-field $(J-E)$ measurements were performed using a SÜSS Microtech probe station equipped with a Keithley 4200 semiconductor characterization system. Fig. 3.7 shows the dielectric constant-electric field curves obtained from the capacitance measurements of the $75 \mathrm{~nm}$ and $100 \mathrm{~nm}$ thick PZT films. The dielectric constant was obtained from the $C-E$ measurements at an ac bias of $30 \mathrm{mV}$ and a switching frequency of $1 \mathrm{kHz}$.

We observe a typical butterfly shaped hysteresis in the dielectric constant versus electric field curves. This is a ferroelectric behavior and indicates the domain re-orientation process inside the PZT layer [30, 31]. However, as observed in the polarization loop experiments, the center of the hysteresis loops has shifted towards the negative bias range. In addition, there is an asymmetry in the maximum dielectric constant values, especially for the capacitor with a $75 \mathrm{~nm}$-thick PZT layer. The shift in hysteresis loops as well as the asymmetry in the dielectric constant could be due to space charge accumulation at the PZT/electrode interface. This can be seen as an additional nonferroelectric or passive layer adjacent to the electrodes, which could have been formed during the fabrication of the capacitor stack [32-37]. 


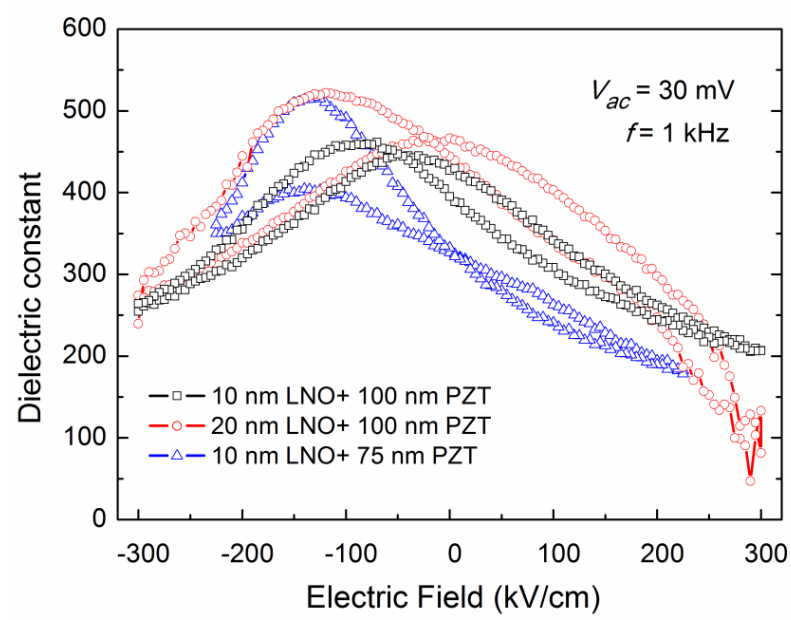

Fig. 3.7 Dielectric constant-electric field curves of the PZT capacitors for various LNO layer and PZT film thicknesses $\left(f=1 \mathrm{kHz}, V_{\mathrm{ac}}=30 \mathrm{mV}\right.$ ). The bottom electrode was grounded and a maximum $\mathrm{dc}$ bias of $3 \mathrm{~V}$ was applied to the top electrode.

Generally, this passive layer can form (i) a graded junction, (ii) an insulating defect layer, (iii) an intermediate compound/phase or (iv) a highly charged layer inside the PZT capacitor [38]. Therefore, the term "effective" is used to describe its properties. This layer can affect both ferroelectric and piezoelectric properties. Such a thin layer could be more prominent when the thickness of the PZT layer decreases, which can explain the thickness dependence of the difference in the maximum dielectric constants.

The problem, as will be shown further in section 3.2.3.3, is that the location is uncertain and the physical thickness of the passive layer is too thin to be detected by any optical or electron-beam technique. Therefore, in our analysis, we assume a single passive layer in the PZT capacitor which is assumed to be present at the Ti/PZT interface. This is because the PZT/LNO interface received a higher temperature treatment than the top Ti/PZT interface, resulting in less extended structural defects for the former [39]. Note that this is one possible way to explain the system. As will be shown later, it is based on a working hypothesis.

\subsubsection{Analysis}

There have been several reports in which the passive layer properties were estimated for different ferroelectric materials $[35,40]$. These have been reported for PZT by M. D. Nguyen [22]. To obtain the dielectric constant of the PZT layer, as well as the properties of the passive layer, the following series capacitor equation is employed [40]: 


$$
\frac{1}{C_{\text {total }}}=\frac{1}{C_{\text {pass }}}+\frac{1}{C_{P Z T}}=\frac{1}{A \varepsilon_{0}}\left(\frac{t_{\text {pass }}}{\varepsilon_{\text {pass }}}+\frac{t_{P Z T}}{\varepsilon_{P Z T}}\right)
$$

where, $\varepsilon_{0}\left(=8.85 \times 10^{-12} \mathrm{~F} / \mathrm{m}\right)$ and $\varepsilon_{\mathrm{PZT}}$ are the vacuum permittivity and relative dielectric constant of the PZT layer, respectively. $\varepsilon_{\text {pass }}$ is the relative dielectric constant of the passive layer between film and electrode and $A$ is the capacitor area, $t_{\mathrm{PZT}}$ and $t_{\text {pass }}$ are the thickness of PZT ferroelectric film and the effective thickness of the passive layer, respectively.

Assuming a relatively thin passive layer thickness, the effective values of relative dielectric constant $\varepsilon_{\text {PZT }}$ and $t_{\text {pass }} / \varepsilon_{\text {pass }}$ are calculated from the inverse capacitance vs. PZT layer thickness $(1 / C-t)$ graph. The $1 / C$ - $t$ curve obtained at zero volt is shown in Fig. 3.8. From the slope and the intercept values of this curve, the effective values of the relative dielectric constant $\varepsilon_{\mathrm{PZT}}$ and $t_{\text {pass }} / \varepsilon_{\text {pass }}$ are calculated to be 600 and $8.95 \times 10^{-11} \mathrm{~m}$, respectively.

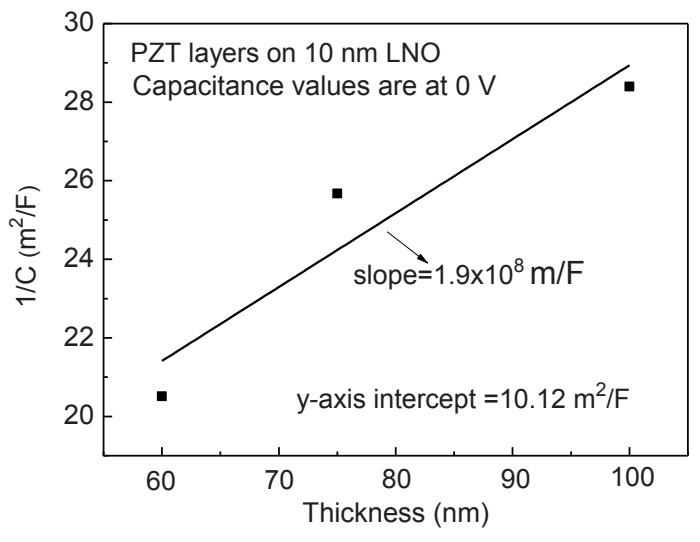

Fig. 3.8 $1 / C$ vs thickness curve at zero volt bias. The LNO layer was $10 \mathrm{~nm}$ thick for all samples.

Additional information on the passive layer is obtained from leakage current densityelectric field $(J-E)$ measurements on the PZT capacitors. Unfortunately, during the PLD process, large clusters of the target material were also deposited. This resulted in an inhomogeneous film and consequently non-uniformly distributed paths in the leakage current. Therefore, the leakage current of the capacitors depends also on these additional leakage paths.

Several capacitors were tested in this work and the ones with a relatively low leakage are plotted. Fig. 3.9 shows the $J$ - $E$ characteristics of the capacitors at room temperature. It is 
observed that the thicker LNO layer gives a lower leakage. This could be due to the growth of a higher quality PZT material.

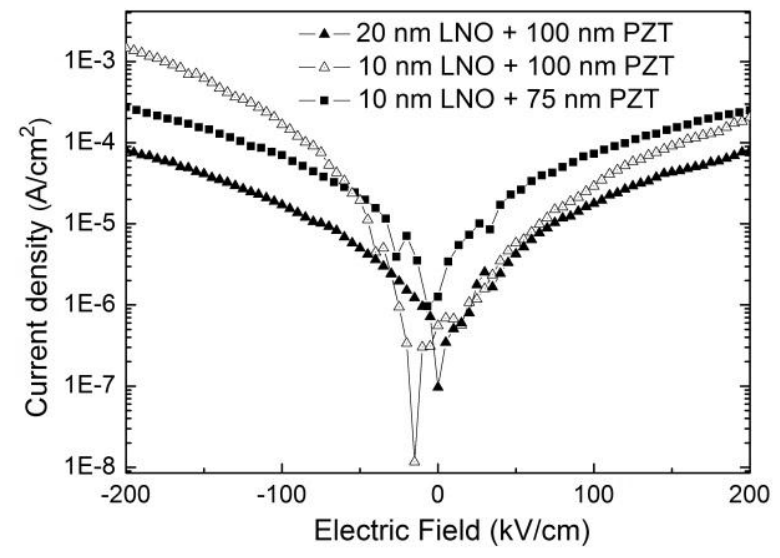

Fig. 3.9 Room temperature $J-E$ characteristics of a $75 \mathrm{~nm}$ and a $100 \mathrm{~nm}$ PZT film on a $10 \mathrm{~nm}$-thick and $20 \mathrm{~nm}$-thick LNO layer.

The Ti and the LNO that contact the PZT layer have a work function of $4.33 \mathrm{eV}$ [41] and $4.60 \mathrm{eV}$ [42], respectively. Since the electron affinity of PZT is around $3.5 \mathrm{eV}$ [38], we expect an asymmetry due to the $0.27 \mathrm{eV}$ difference in Schottky barrier height (SBH) between top and bottom contacts for the 'ideal' PZT capacitor. The minimum SBH would be that at the Ti/PZT interface: $0.83 \mathrm{eV}$. However, the $J-E$ curves are almost symmetric for forward and reverse biases. This behavior can be attributed to the existence of interface states at the contacts, resulting in barrier alignment [43], yielding symmetric J-E curves.

Furthermore, in our work, the PZT layer is intentionally undoped and relatively thin, meaning that the electrodes govern the electrostatics. To see the effect of doping in the band alignment of PZT structure, TCAD (technology computer aided design) simulations [44] were performed ${ }^{2}$ as shown in Fig. 3.10.

Since the differences between the electron affinity of PZT and the work functions of Ti and LNO are relatively low compared to half the band gap of PZT $\left(E_{G}=3.2 \mathrm{eV}\right)$, the leakage current of the PZT system under study is an electron current, i.e. n-type conduction.

\footnotetext{
${ }^{2}$ Simulations were performed by R.J.E. Hueting, with courtesy.
} 

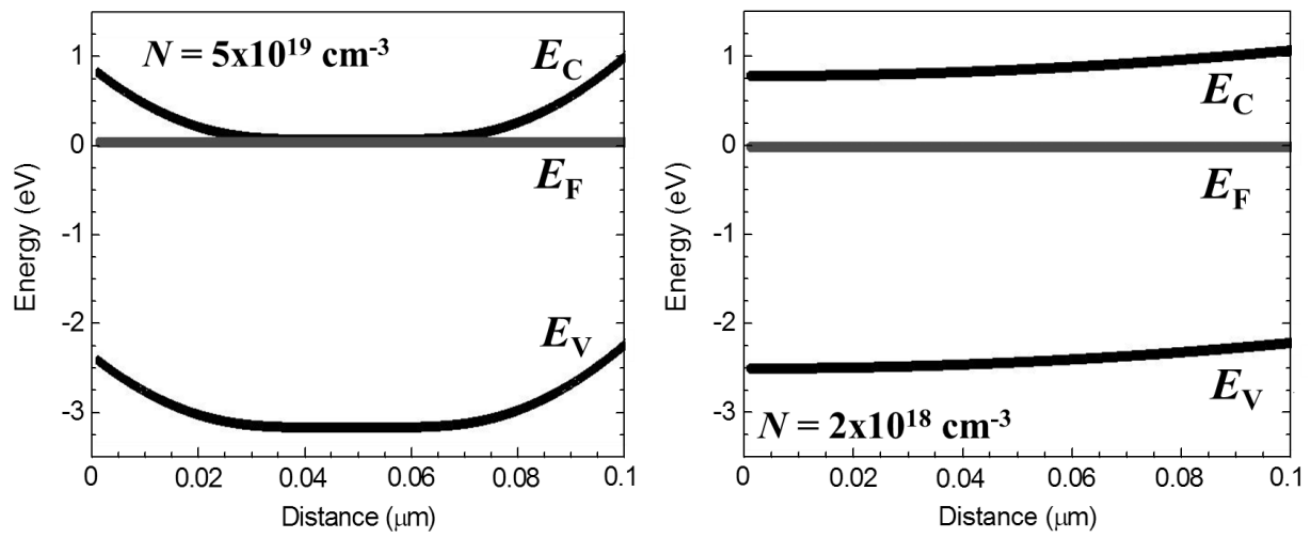

Fig. 3.10 The energy band diagram of the $100 \mathrm{~nm}$ thick PZT layer with (a) $5 \times 10^{19} \mathrm{~cm}^{-3}$ and (b) $2 \times 10^{18}$ $\mathrm{cm}^{-3}$ doping. The Ti/PZT interface (left) and PZT/LNO interface (right) give a barrier height of 0.8 and $1.1 \mathrm{eV}$, respectively.

By inserting the experimental layer thickness and low doping level (less than $2 \times 10^{18} \mathrm{~cm}^{-3}$ ), a full depletion of the PZT layer was predicted by the simulator. Therefore, although linear behavior of the In $J-\sqrt{E}$ graph in our capacitors (not shown) indicates Schottky type emission, it is questionable whether the current conduction can be explained by pure Schottky emission. This is because of fully depleted layers comprising a passive layer at the interface with almost symmetric J-E curves. In addition, as will be shown later in Fig. 3.11, the weak temperature dependence of the current density is an indication that the $\mathrm{SBH}$ is lower than expected.

Based on the diffusion theory in metal-semiconductor contacts [9], it can be derived from the drift-diffusion equations that:

$$
\begin{gathered}
J=q N_{C} \mu_{n} u_{T}\left[\exp \left(\frac{V}{u_{T}}\right)-1\right] / \int_{0}^{t} \exp \left[\frac{E_{C}(x)}{k T}\right] d x, \text { with } \\
N_{C}=2\left(\frac{2 \pi m_{e f f} k T}{h^{2}}\right)^{3 / 2}
\end{gathered}
$$

where $T$ is the temperature, $q$ is the elementary charge, $t$ is the total layer thickness, $\mu_{n}$ is electron mobility, $u_{T}$ is thermal voltage $(=k T / q), k$ is Boltzmann's constant, $N_{C}$ is the conduction band effective density of states, $m_{\text {eff }}$ is the effective mass, $h$ is Planck's constant, and $E_{C}(x)$ is the conduction band energy depending on the distance $x$.

Using Eq. (3.2) for an ideal asymmetric metal-ferroelectric-metal structure in full depletion mode, we obtain: 


$$
J=q N_{C} \mu_{n}\left(\frac{\varphi_{B 1}-V-\varphi_{B 2}}{t_{t}}\right) \frac{\exp \left(\frac{V}{u_{T}}\right)-1}{\exp \left(\frac{\varphi_{B 1}}{u_{T}}\right)-\exp \left(\frac{\varphi_{B 2+V}}{u_{T}}\right)}
$$

where $\varphi_{B 1}$ and $\varphi_{B 2}$ are the barrier heights at the Ti/PZT and PZT/LNO interfaces, respectively. Depending on the differences in the barrier heights, the reverse and forward current will be different. In Fig. 3.11, the current density is plotted against the applied voltage showing that the model (Eq. (3.3a)) is in good agreement with TCAD simulations. The results also show a large asymmetry in the $I-V$ curve for a positive and a negative bias.

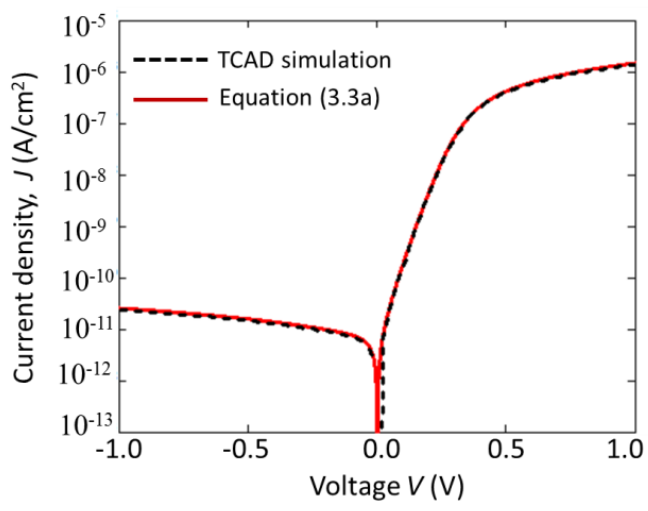

Fig. 3.11 J-V graph of the PZT capacitor structure obtained from TCAD simulations and from the model (Eq. 3.3a).

As stated before, the barrier heights seem to be almost equal, $\varphi_{B 2}=\varphi_{B 1}=\varphi_{B}$, possibly due to interface states. From Eq. (3.3a) we can see that for this case the equation reduces to:

$$
J=q N_{C} \mu_{n}\left(\frac{V}{t_{t}}\right) \exp \left(-\frac{\varphi_{B}}{u_{T}}\right)
$$

This equation shows a weak voltage dependency and is symmetric. However, the image force lowering effect is primarily present at the interface, and the passive layer is not included here. This affects the integral of the conduction band energy, see Eq. (3.2). Therefore, the following leakage current equation, developed by Simmons, applies for PZT capacitors $[38,45]$ :

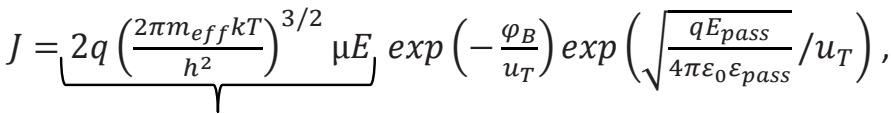

$$
\begin{aligned}
& K(V)=\alpha V
\end{aligned}
$$


where $\varphi_{B}$ is the $\mathrm{SBH}, \varepsilon_{0}$ is vacuum dielectric constant, $\varepsilon_{\text {pass }}$ is the dielectric constant of the passive layer, and $E$ is the applied field across the PZT layer. This model is comparable with Eq. (3.3b); however, it has an additional exponential term. This exponential term determines the charge injection at the electrode-ferroelectric interface and $E_{\text {pass }}$ represents the field at the interface. Eq. (3.4) describes that the injection is interfacecontrolled, while the transport is a bulk-controlled phenomenon, i.e. diffusion, governed by the integral of the potential barrier over the PZT film thickness.

The properties of the passive layer can be found using Eq. (3.4), where the slope of the In $J-\sqrt{V}$ graph is equal to $q \sqrt{q / 4 \pi \varepsilon_{0} \varepsilon_{\text {pass }} t_{\text {pass }}} / k T$. This method has been used before in other studies using the pure Schottky emission equation $[35,40]$. However, in this case $V$ $=V_{\text {pass }}$, the voltage drop across the passive layer, and the relation between $V$ and $V_{\text {pass }}$ needs to be determined. This relation can be found by writing the electric displacement at the PZT film-passive layer interface. The electric displacement in PZT can be described as $D=\varepsilon E$ by assuming that the polarization of the material is linearly dependent on the field, which holds especially at low fields. At a boundary between the passive layer and PZT film, the displacement field is continuous, i.e. $\varepsilon_{\mathrm{PZT}} E_{\mathrm{PZT}}=\varepsilon_{\text {pass }} E_{\text {pass }}$.

Therefore, $V_{P Z T}$ can be written as:

$$
V_{P Z T}=\varepsilon_{\text {pass }} V_{\text {pass }} t_{P Z T} /\left(\varepsilon_{P Z T} t_{\text {pass }}\right) .
$$

The applied voltage is the sum of the voltage drop over the passive layer and over the PZT $\left(V=V_{\text {pass }}+V_{P Z T}\right)$. Since $\varepsilon_{\mathrm{PZT}}=600$ and $t_{\text {pass }} / \varepsilon_{\text {pass }}=8.95 \times 10^{-11} \mathrm{~m}, V$ can be estimated to be $2.86 \times V_{\text {pass }}$ from Eq. (3.5). The slope of In $J-\sqrt{V_{\text {pass }}}$ curve is 6.74 and hence $t_{\text {pass }} \varepsilon_{\text {pass }}=47.26$ $\mathrm{nm}$. Therefore, employing capacitance and leakage current measurements, the effective passive layer thickness and its relative dielectric constant are estimated to be $2.1 \mathrm{~nm}$ and 23, respectively. Such a thin passive layer is difficult to observe with any physical technique. Moreover, the effective electrical thickness might be different from the actual physical layer thickness.

The SBH can be extracted from temperature dependent current density - electric field ( $J$ -E) measurements. Fig. 3.12 shows the temperature dependent leakage characteristics of capacitors with $60 \mathrm{~nm}, 75 \mathrm{~nm}$ and $100 \mathrm{~nm}$ thick PZT layers. 

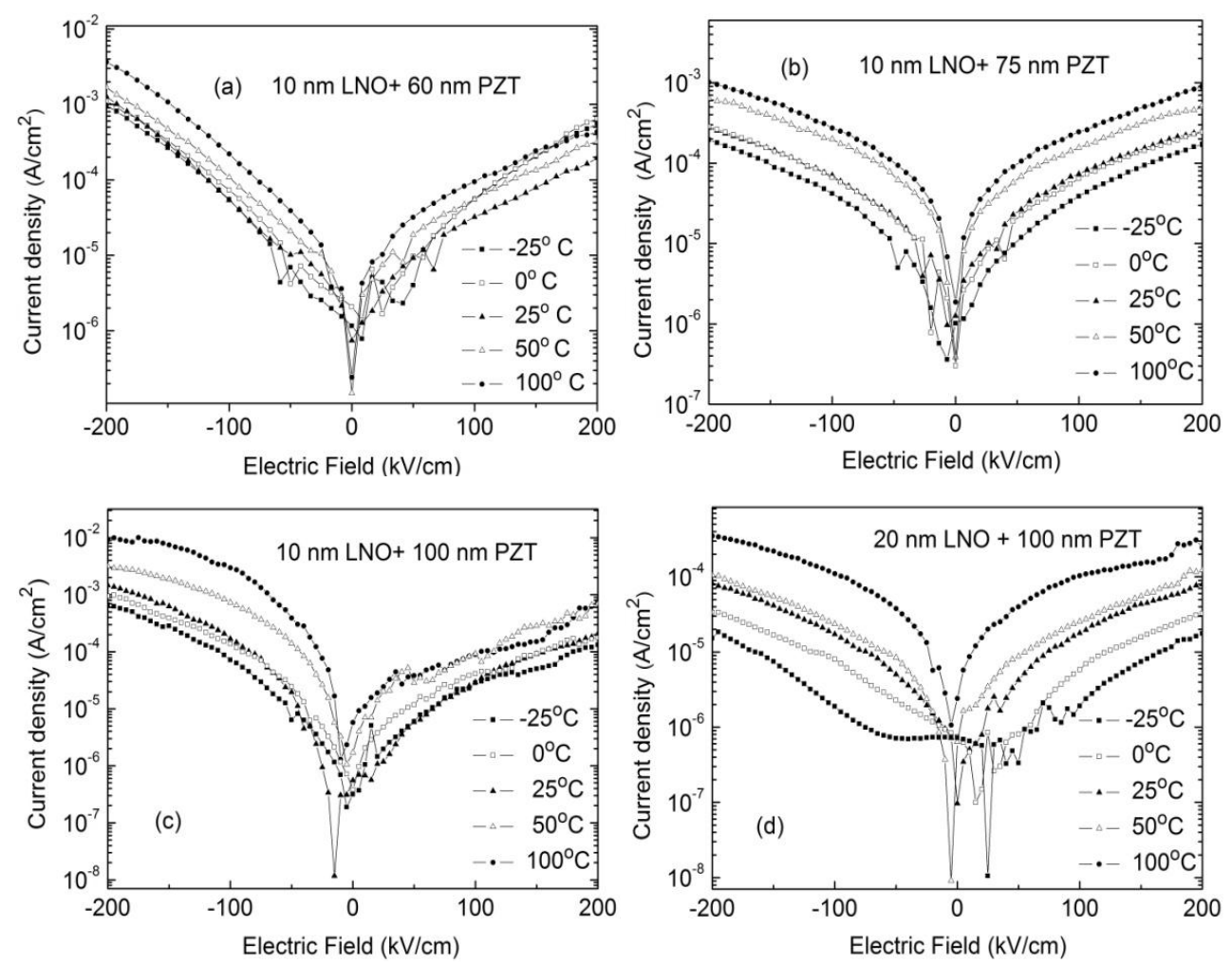

Fig. 3.12 Temperature dependent J-E characteristics of (a) a $60 \mathrm{~nm}$ (b) a $75 \mathrm{~nm}$ (c) a $100 \mathrm{~nm}$ PZT film on a $10 \mathrm{~nm}$-thick LNO layer and (d) a $100 \mathrm{~nm}$-thick PZT film on a $20 \mathrm{~nm}$ thick LNO layer.

The temperature dependency of the leakage current is different for each sample. A weak temperature dependency is observed, especially for thin PZT layers. The sample with the lowest leakage and a $T^{3 / 2}$ temperature dependency, presented in Fig. 3.12 (d), was chosen for the SBH extraction.

For this purpose, $\ln \left(J / T^{3 / 2}\right)$ curves have been plotted against $1 / T$ in Fig. 3.13 (a) for different constant forward bias voltages. The slope of these curves equals to $-q\left(\varphi_{B}-\sqrt{q E_{\text {pass }} / 4 \pi \varepsilon_{0} \varepsilon_{\text {pass }}}\right) / k$. Inserting the parameters we calculated before, we obtain for the SBH at the Ti/PZT interface $q \varphi_{B}=0.32 \mathrm{eV}$. As mentioned before the expected minimum SBH would have been around $0.8 \mathrm{eV}$. The measured low (apparent) barrier height however can be attributed to ferroelectric polarization induced barrier lowering as reported by Pintilie et al. [39].

Fig. 3.13 (b) and Fig. 3.13 (c) show the measurements of the current density as a function of the applied electric field (symbols). The solid lines represent theoretical fit using Eq. (3.4), keeping the factor $\alpha$ of the voltage dependent pre-exponential term $K(V)=\alpha V$ as a fitting parameter. This parameter $(\alpha)$ is in the same order as reported by Pintilie et al. 
[39], i.e. $10^{-5} \mathrm{~A} / \mathrm{Vcm}^{2}$. Hence, there is a good agreement between the measurements and the theoretical fit both for reverse and forward bias conditions. Therefore, the barrier heights for bottom and top interfaces are the same in our $20 \mathrm{~nm} \mathrm{LNO} \mathrm{+} 100 \mathrm{~nm}$ PZT capacitor structures.
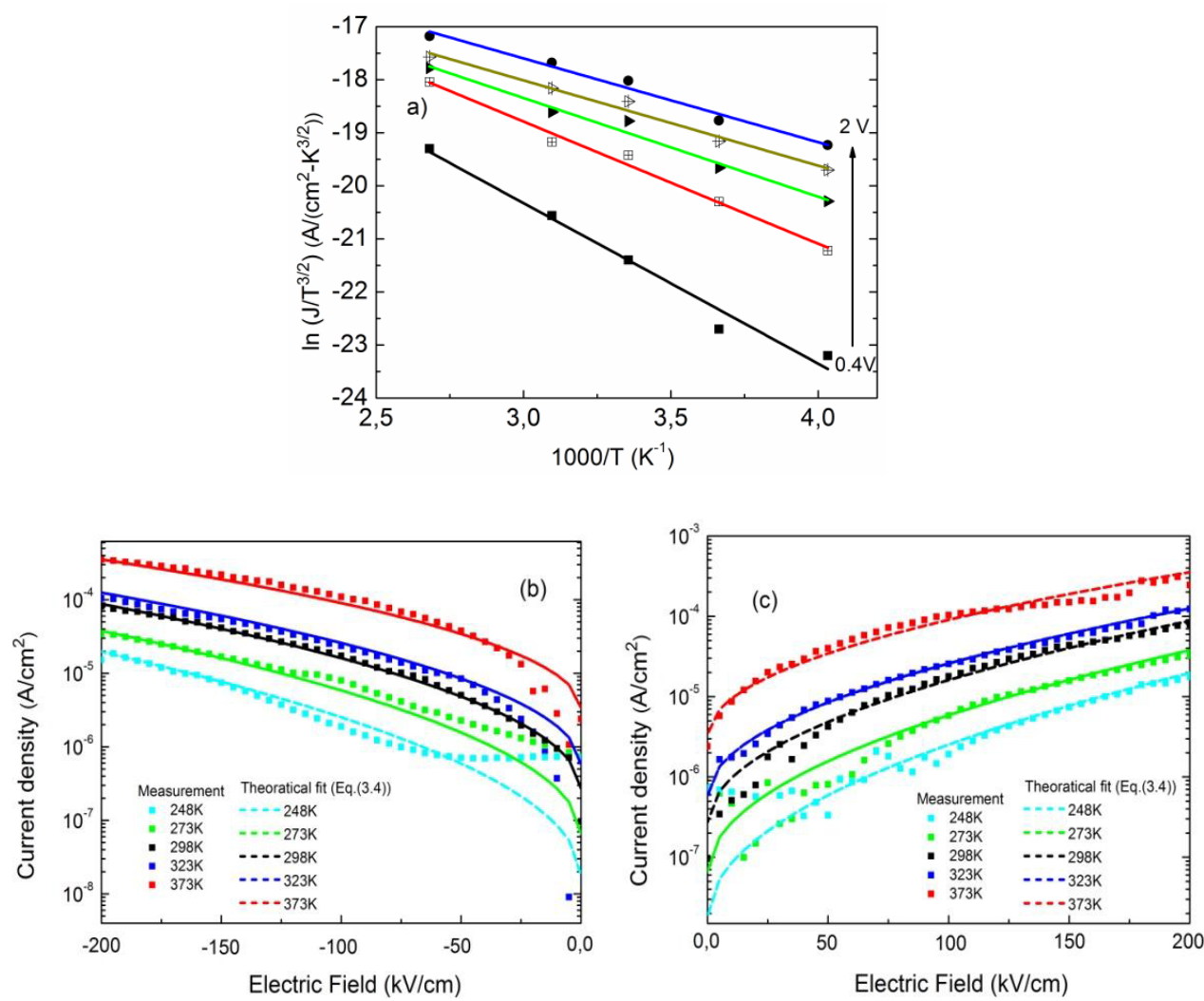

Fig. 3.13 (a) In $\left(J / T^{3 / 2}\right)$ plotted against $1 / T$ for different constant forward bias voltages, (b) and (c) measured J-E curves (symbols) and theoretical fit (dotted lines) for $20 \mathrm{~nm}$ LNO + $100 \mathrm{~nm}$ PZT film for different measurement temperatures at reverse and forward bias, respectively.

\subsection{Conclusions}

In this chapter, thin-film PZT/LNO stacks with different layer thicknesses deposited on an encapsulated TiN electrode were studied. XRD analysis evidenced (100)/(001) and $(110) /(101)$ orientations of the PZT layer and the results obtained from the XPS analysis show no significant diffusion of atoms between the different layers, which is important for the device behavior. We obtained a promising effective piezoelectric coefficient $d_{33, f}$ reaching a maximum value of $53 \mathrm{pm} / \mathrm{V}$. The high $d_{33, \mathrm{f}}$ value favors PZT as a stressor for device structures like steep sub-threshold switches. In addition, an average remnant 
polarization $P_{r}$ of $19 \mu \mathrm{C} / \mathrm{cm}^{2}$ is obtained. A shift in the $P$-E hysteresis loops as well as an asymmetry in the dielectric constant against the electric field was observed.

Based on the electrical analysis, the properties of the $100 \mathrm{~nm}$ thick PZT layer and PZTelectrode interface were determined. Although a $0.27 \mathrm{eV}$ difference in SBH between top and bottom contacts is expected for the 'ideal' PZT capacitors, the J-E curves obtained from thin-film PZT capacitors are almost symmetric for forward and reverse biases. Together with a shift and an asymmetry mentioned before, this effect can be an indication of an additional non-ferroelectric or passive layer adjacent to the electrodes. This layer can result in barrier alignment and yield symmetric $J-E$ curves.

Further analysis was done by capacitance and leakage current measurements to obtain dielectric properties of PZT and passive layer. It is found that the relative dielectric constant of the PZT layer has a value of 600 whereas the effective thickness and the relative dielectric constant of the passive layer are estimated to be $2.1 \mathrm{~nm}$ and 23, respectively. In addition, a low apparent barrier height of $0.32 \mathrm{eV}$ was obtained, possibly due to ferroelectric polarization induced barrier lowering.

The obtained values have been inserted into the leakage current equation and good fitting has been obtained between the measurements and the model (see Fig. 3.13 (b) and (c)). Therefore, our analysis on sub-100 nm PZT layers shows that the leakage current can be described with a diffusion-based rather than a pure Schottky emission model. Furthermore, the leakage current is governed by interface controlled injection and its transport is diffusion-limited.

\section{References}

[1] W. Känzig, "Ferroelectrics and Antiferroelectrics", Solid State Physics, 4, Academic Press, ISBN 0126077045, New York, 1957

[2] M. Klee, D. Wissen, W. Keur, R. Kiewitt, D. Bausen, and P. Lok, "Oxide films for integrated capacitors in thin film functional modules", Material Research Society, 655, pp. CC13.1.1-CC13.1.11, 2001.

[3] P. Muralt, "Ferroelectric thin films for micro sensors and actuators", J. Micromech Microeng. 10, pp. 136-146, 2000.

[4] N. Setter, D. Damjanovic, L. Eng, G. Fox, S. Gevorgian, S. Hong, A. Kingon, H. Kohlstedt, N. Y. Park, G. B. Stephenson, I. Stolitchnov, A. K. Tagantsev, D.V. Taylor, T. Yamada, and S. Streiffer, "Ferroelectric thin films: Review of materials, properties, and applications", Journal of Applied Physics, 100, pp. 051606-051606-46, 2006.

[5] C. A. Paz de Araujo, J. D. Cuchiaro, L. D. McMillan, M. Scott, J. F. Scott, "Fatigue-free ferroelectric capacitors with platinum electrodes", Nature, 374, pp. 627-629, 1995.

[6] J. F. Scott, Ferroelectric Memories (Springer, New York), 2000.

[7] S. Salahuddin, and S. Datta, "Use of Negative Capacitance to Provide Voltage Amplification for Low Power Nanoscale Devices", Nanoletters, 8, pp. 405-410, 2008. 
[8] A. Rusu, G.A. Salvatore, D. Jimenez, and A. M. Ionescu, "Metal-Ferroelectric-Meta-Oxidesemiconductor field effect transistor with sub-60mV/decade subthreshold swing and internal voltage amplification", Technical Digest International Electron Devices Meeting (IEDM), pp. 16.3.116.3.4, 2010.

[9] S. M. Sze, K. K. Ng, "Physics of Semiconductor Devices", $3^{\text {rd }}$ edition, John Wiley \& Sons, pp. 158-160, 2007.

[10] D. Newns, B. Elmegreen, H. Liu, and G. Martyna, "A low-voltage high-speed electronic switch based on piezoelectric transduction", Journal of Applied Physics, 111, pp. 084509, 2012.

[11] T. van Hemert, and R. J. E. Hueting, "Active Strain Modulation in Field Effect Devices", Proc. of the European Solid-State Device Research Conference (ESSDERC), pp. 125-128, 2012.

[12] A. K. Singh, S. K. Mishra, Ragani, D. Pandey, S. Yoon, S. Baik and N. Shin, "Origin of high piezoelectric response of $\mathrm{PbZr}_{x} \mathrm{Ti}_{1-\mathrm{x}} \mathrm{O}_{3}$ at the morphotropic phase boundary: Role of elastic instability", Applied Physics Letters, 92, pp. 022910-022913, 2008.

[13] Y. Cao, G. Sheng, J. X. Zhang, S. Choudhury, Y. L. Li, C. A. Randall and L. Q. Chen, "Piezoelectric response of single-crystal $\mathrm{PbZr}_{1-\mathrm{x}} \mathrm{Ti}_{\mathrm{x}} \mathrm{O}_{3}$ near morphotropic phase boundary predicted by phase-field simulation", Applied Physics Letters, 97, pp. 252904-252907, 2010.

[14] H. He, "Phase Transitions in Tin-modified Lead Zirconate Titanate Antiferroelectric Ceramics", PhD thesis, lowa State University, 2007.

[15] C. H. Lin, P. A. Friddle, C. H. Ma, and H. D. Chen, "Effects of thickness on the electrical properties of metalorganic chemical vapour deposited $\mathrm{Pb}(\mathrm{Zr}, \mathrm{Ti}) \mathrm{O}_{3}(25-100 \mathrm{~nm})$ thin films on $\mathrm{LaNiO}_{3}$ buffered $\mathrm{Si}^{\prime}$, Journal of Applied Physics, 90, pp. 1509-1515, 2001.

[16] E. Defay, T. Lacrevaz, T. T. Vo, V. Sbrugnera, and C. Bermond, "Ferroelectric properties of $\mathrm{Pb}(\mathrm{Zr}, \mathrm{Ti}) \mathrm{O}_{3}$ thin films until $40 \mathrm{GHz}$ ", Applied Physics Letters, 94, pp. 052901- 052901-3, 2009.

[17] J. Li, P. Li, G. Zhang, J. Yu, Y. Wu, X. Wen, "The thickness effect of $\mathrm{Bi}_{3.25} \mathrm{La}_{0.75} \mathrm{Ti}_{3} \mathrm{O}_{12}$ buffer layer in $\mathrm{PbZr}_{0.58} \mathrm{Ti}_{0.42} \mathrm{O}_{3} / \mathrm{Bi}_{3.25} \mathrm{La}_{0.75} \mathrm{Ti}_{3} \mathrm{O}_{12}$ (PZT/BLT) multilayered ferroelectric thin films", Thin Solid Films, 519, pp. 6021-6025, 2011.

[18] Q. Zou, H. E. Ruda, B. G. Yacobi, "Improved dielectric properties of lead zirconate titanate thin films deposited on metal foils with $\mathrm{LaNiO}_{3}$ buffer layers", Applied Physics Letters, 78, pp. 1282-1284, 2001.

[19] N. Sama, R. Herdier, D. Jenkins, C. Soyer, D. Remiens, M. Detalle, and R. Bouregba, "On the influence of the top and bottom electrodes -a comparitive study between Pt and LNO electrodes for PZT thin films", Journal of Crystal Growth, 310, pp. 3299-3302, 2008.

[20] R. N. Torah, S. P. Beeby, and N. M. White, "Experimental investigation into the effect of substrate clamping on the piezoelectric behavior of thick-film PZT elements", Journal of Physics D: Applied Physics, 37, pp. 1074-1078, 2004.

[21] G. Vellianitis, M. J. H. van Dal, L. Witters, G. Curatola, G. Doornbos, N. Collaert, C. Jonville, C. Torregiani, L. S. Lai, J. Petty, B. J. Pawlak, R. Duffy, M. Demand, S. Beckx, S. Mertens, A. Delabie, T. Vandeweyer, C. Delvaux, F. Leys, A. Hikavyy, R. Rooyackers, M. Kaiser, R. G. Weemaes, F. Voogt, H. Roberts, D. Donnet, S. Biesemans, M. Jurczak, and R. J. R. Lander, "Gatestacks for scalable highperformance FinFETs", Technical Digest International Electron Devices Meeting (IEDM), pp. 681684, 2007.

[22] M. D. Nguyen, "Ferroelectric and Piezoelectric properties of epitaxial PZT films and devices on Silicon" PhD thesis, University of Twente, 2010.

[23] B. Kaleli, M. D. Nguyen, J. Schmitz, R. A. M. Wolters, R. J. E. Hueting, "Analysis of ferroelectric and electrical properties of sub-100 nm thick PZT/LNO stacks on an encapsulated TiN electrode", International Conference on Micro and Nano Engineering, 2013.

[24] M. Tsukada and H. Yamawaki and M. Kondo, "Crystal structure and polarization phenomena of epitaxially grown $\mathrm{Pb}(\mathrm{Zr}, \mathrm{Ti}) \mathrm{O}_{3}$ thin-film capacitors", Applied Physics Letters, 83, pp. 4393-4395, 2003. 
[25] K. Nagashima, M. Aratani and H. Funakubo, "Orientation dependence of ferroelectricity of epitaxially grown $\mathrm{PbZr}_{x} \mathrm{Ti}_{1-\mathrm{x}} \mathrm{O}_{3}$ thin films prepared by metalorganic chemical vapor deposition", Journal of Applied Physics, 89, pp. 4517-4522, 2001.

[26] A. L. Kholkin and Ch. Wutchrich and D. V. Taylor and N. Setter, "Interferometric measurements of electric field-induced displacements in piezoelectric thin films", Review of Scientific Instruments, 67, pp. 1935-1941, 1996.

[27] W. Y. Pan and L. E. Cross, "A sensitive double beam laser interferometer for studying high-frequency piezoelectric and electrostrictive strains", Review of Scientific Instruments, 60, pp. 2701-2705, 1989.

[28] http://www.polytec.com/eu/solutions/vibration-measurement/basic-principles-of-vibrometry/

[29] R. Herdier, D. Jenkins, E. Dogheche, D. Rèmiens, and M. Sulc, "Laser Doppler vibrometry for evaluating the piezoelectric coefficient $d_{33}$ on thin film", Review of Scientific Instruments, 77, pp. 093905-1-5, 2006.

[30] Y. Masuda, "Variation of dielectric constant affected by domain structure and electric-filed-induced strain in ferroelectric ceramics", Japanese Journal of Applied Physics, 33, 1994, pp. 5549-5554, 1994.

[31] H. B. Sharma, H. N. K. Sarma, A. Mansingh, "Fatigue in sol-gel derived barium titanate films", Journal of Applied Physics, 85, pp. 341-345, 1999.

[32] A. K. Tagantsev and G. Gerra, "Interface-induced phenomena in polarization response of ferroelectric thin films", Journal of Applied Physics, 100, pp. 051607-1-35, 2006.

[33] K. T. Kim, C. I. Kim, S. G. Lee, "Ferroelectric properties of $\mathrm{Pb}(\mathrm{Zr}, \mathrm{Ti}) \mathrm{O}_{3}$ heterolayered thin films for FRAM applications", Microelectronic Engineering, 66, pp. 662-669, 2003.

[34] P. Zubko and D. J. Jung and J. F. Scott, "Space charge effects in ferroelectric thin films", Journal of Applied Physics, 100, pp. 114112-1-10, 2006.

[35] H. Yang, "Effective thickness and dielectric constant of interfacial layers of $\mathrm{Pt} / \mathrm{Bi}_{3.15} \mathrm{Nd}_{0.85} \mathrm{Ti}_{3} \mathrm{O}_{12} / \mathrm{SrRuO}_{3}$ capacitors", Applied Physics Letters, 90, pp.232909-1-4, 2007.

[36] Y. Y. Liu, and J. Y. Li, "Space charge and size effects in semiconducting ferroelectric $\mathrm{BaTiO}_{2} / \mathrm{SrTiO}_{3}$ superlattices" Applied Physics Letters, 97, pp. 042905-1-4, 2010.

[37] Y. Wang, F. Yuan, T. Wei, C. Zhu, and K. F. Wang, "Identification of interfacial and bulk effects in modulating fatigue behaviors of $\mathrm{Pb}\left(\mathrm{Zr}_{0.52} \mathrm{Ti}_{0.48}\right) \mathrm{O}_{3}$ thin films", Applied Physics Letters, 101, pp. 0616101-3, 2007.

[38] J. F. Scott, K. Watanabe, A. J. Hartmann, and R. N. Lamb, "Device models for PZT/Pt, BST/Pt, SBT/Pt, and SBT/Bi ferroelectric memories", Ferroelectrics, 225, pp. 83-90, 1999.

[39] L. Pintilie, I. Vrejoiu, D. Hesse, G. LeRhun, and M. Alexe," Ferroelectric polarization-leakage current relation in high quality epitaxial $\mathrm{PbZrTiO}_{3}$ films", Physical Review B, 75, pp. 104103-1-14, 2007.

[40] B. Chen, "Thickness and dielectric constant of dead layer in $\mathrm{Pt} /\left(\mathrm{Ba}_{0.7} \mathrm{Sr}_{0.3}\right) \mathrm{TiO}_{3} / \mathrm{YBa}_{2} \mathrm{Cu}_{3} \mathrm{O}_{7}-\mathrm{X}$ capacitor", Applied Physics Letters, 84, pp. 583-585, 2004.

[41] H. B. Michaelson, "The work function of the elements and its periodicity", Journal of Applied Physics, 48, pp. 4729-4733, 1977.

[42] F. Yan, I. Sterianou, S. Miao, I.M. Reany, M.O. Lai, and L. Lu, "Multiferroic properties of $\mathrm{Bi}\left(\mathrm{Fe}_{0.5} \mathrm{Sc}_{0.5}\right) \mathrm{O}_{3}-\mathrm{PbTiO}_{3}$ thin films", Physica Scripta, T139, pp. 014003-1-4, 2010.

[43] J. Robertson, "Band offsets of high dielectric constant gate oxides on silicon", Journal of NonCrystalline Solids", 303, pp.94-100, 2002.

[44] ATLAS, Device simulation software, version 5.18.3.R, Silvaco, Inc., 2012

[45] J. G. Simmons, "Richardson-Schottky effect in solids", Physical Review Letters, 15, pp. 967-968, 1965. 


\section{Fabrication and characterization of FinFETs}

This chapter presents a fabrication scheme of FinFET devices realized at the MESA+ Research Institute, Twente. The devices and the process control test structures were characterized both electrically and optically. It is shown that the fabricated FinFETs have a well-defined body down to $20 \mathrm{~nm}$ wide dimensions and an aspect ratio up to 7.5. The functional devices show promising performance with near-ideal sub-threshold swing $1 \approx$ $75 \mathrm{mV} / \mathrm{dec}$ ) and high on-current off-current ratio $I_{\text {on }} / I_{\text {off }}$ of around $10^{8}$. The devices presented in this chapter pave the way for the novel steep subthreshold devices, discussed in Chapter 5. 


\subsection{Fabrication of FinFETs}

\subsubsection{Design}

FinFETs consist of fins, S/D areas and metal contacts. Because of improved gate control (see section 1.2), FinFETs with sub-tens of nanometer fin width ( $\left.W_{\text {FIN }}\right)$ are desired. The devices designed in this work comprise fin widths from $15 \mathrm{~nm}$ to $1000 \mathrm{~nm}$. To process these aggressive dimensions as well as features in micron range for contact regions, both an electron beam system (the Raith 150-TWO) and optical contact lithography was used.

Devices with different number of fins, $W_{\text {FIN }}$ and gate length $\left(L_{\text {gate }}\right)$ were designed with the Cadence mask design software. Fig. 4.1 shows the schematic mask layout of a typical FinFET including its design parameters. All the designed devices comprise either a single fin or 5 parallel fins. The designed pitch for the FinFETs is $1 \mu \mathrm{m}$ except for the $1 \mu \mathrm{m}$ wide devices having a pitch of $3 \mu \mathrm{m}$. Source-Drain extensions $\left(L_{s d}\right)$ were designed as $15-23 \mu \mathrm{m}$ long. An overview of the designed mask dimensions is given in Table 4.1.

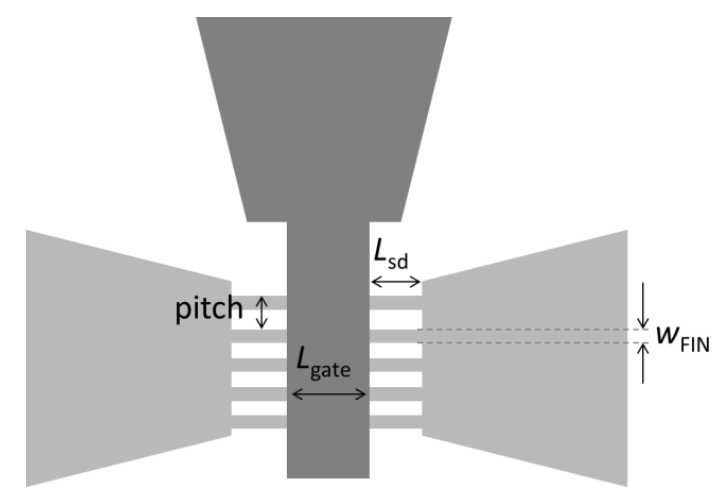

Fig. 4.1 Schematic mask layout of the FinFET with its design parameters.

Table 4.1 Summary of the designed FinFET mask dimensions

\begin{tabular}{lcccccccc}
\hline \hline & $\boldsymbol{W}_{\text {FIN }}$ & $\boldsymbol{W}_{\text {FIN }}$ & $\boldsymbol{W}_{\text {FIN }}$ & $\boldsymbol{W}_{\text {FIN }}$ & $\boldsymbol{W}_{\text {FIN }}$ & $\boldsymbol{W}_{\text {FIN }}$ & $\boldsymbol{W}_{\text {FIN }}$ & $\boldsymbol{W}_{\text {FIN }}$ \\
\hline \hline $\boldsymbol{L}_{\text {gate }}=\mathbf{2} \boldsymbol{\mu m}$ & $15 \mathrm{~nm}$ & $20 \mathrm{~nm}$ & $30 \mathrm{~nm}$ & $40 \mathrm{~nm}$ & $50 \mathrm{~nm}$ & $60 \mathrm{~nm}$ & $100 \mathrm{~nm}$ & $1000 \mathrm{~nm}$ \\
$\boldsymbol{L}_{\text {gate }}=\mathbf{3} \boldsymbol{\mu m}$ & $15 \mathrm{~nm}$ & $20 \mathrm{~nm}$ & $30 \mathrm{~nm}$ & $40 \mathrm{~nm}$ & $50 \mathrm{~nm}$ & $60 \mathrm{~nm}$ & $100 \mathrm{~nm}$ & $1000 \mathrm{~nm}$ \\
$\boldsymbol{L}_{\text {gate }}=\mathbf{1 0} \boldsymbol{\mu m}$ & $15 \mathrm{~nm}$ & $20 \mathrm{~nm}$ & $30 \mathrm{~nm}$ & $40 \mathrm{~nm}$ & $50 \mathrm{~nm}$ & $60 \mathrm{~nm}$ & $100 \mathrm{~nm}$ & $1000 \mathrm{~nm}$ \\
$\boldsymbol{L}_{\text {gate }}=\mathbf{2 0} \boldsymbol{\mu m}$ & $15 \mathrm{~nm}$ & $20 \mathrm{~nm}$ & $30 \mathrm{~nm}$ & $40 \mathrm{~nm}$ & $50 \mathrm{~nm}$ & $60 \mathrm{~nm}$ & $100 \mathrm{~nm}$ & $1000 \mathrm{~nm}$ \\
$\boldsymbol{L}_{\text {gate }}=\mathbf{3 0} \boldsymbol{\mu m}$ & $15 \mathrm{~nm}$ & $20 \mathrm{~nm}$ & $30 \mathrm{~nm}$ & $40 \mathrm{~nm}$ & $50 \mathrm{~nm}$ & $60 \mathrm{~nm}$ & $100 \mathrm{~nm}$ & $1000 \mathrm{~nm}$ \\
\hline \hline
\end{tabular}

Process control test structures were also designed for specific purposes. The Greek Cross (GC) structure [1] is commonly used to measure the sheet resistance of the layers. In this 
GC structure, the current $(I)$ is forced between two nearby positioned contacts and the voltage drop $(V)$ over the central square is measured using the other two contacts (see e.g. Fig. 4.2). The measured resistance $(R)$ is $V /$ I. If $L>2 W$, the size and shape of the contacts does not have an effect on the potential distribution and the structure fulfill the Van der Pauw boundary conditions. A correction factor $\pi / \ln (2)$ is used to extract sheet resistance $\left(R_{\text {sh }}\right)$ [1-3]. Therefore,

$$
R_{s h}=\frac{\pi}{\ln (2)} R \text {. }
$$

As shown in Fig. 4.2, the GC structures were used for three different purposes in this work: to determine $i$ ) the doping concentration in the S/D extension regions from the resistance measurement, ii) the sheet resistance of the gate material, and iii) the sheet resistance of the channel. In Fig. 4.2 (a), the GC composed of two doped-Si arms. This structure was used to address point i). In Fig. 4.2 (b), both arms of the structure compose gate layers (poly-Si/TiN) to measure the sheet resistance of the gate material (point ii)). Fig. 4.2 (c) shows the GC with one arm $\mathrm{Si}$ and the other arm gate material. During processing, this structure faced the same treatment as the FinFETs. Therefore, the Si under the gate is supposed to be undoped whereas the rest of $\mathrm{Si}$ arm is doped like the S/D extensions. Using this test structure, the channel resistance can be measured to identify whether the masking was successful in keeping the channel un-doped. Table 4.2 shows the width $(W)$ and length $(L)$ dimensions of the GCs.
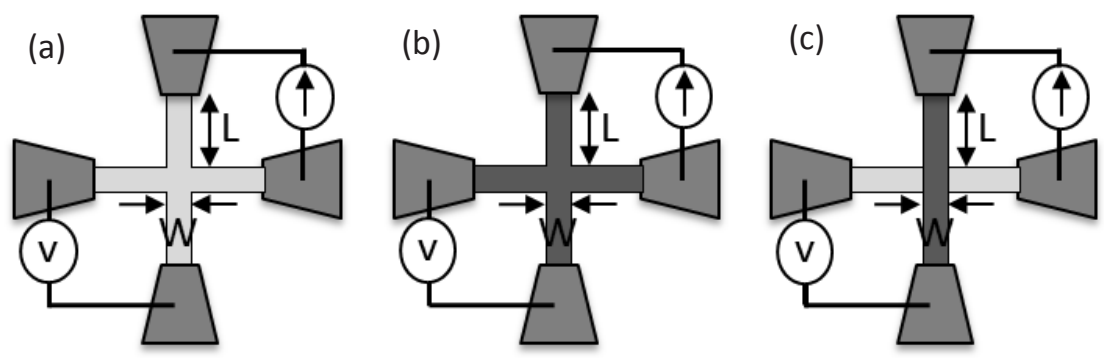

Fig. 4.2 The GC structure comprising (a) doped Si arms (GC I) (b) poly-Si/TiN arms (GC II) (c) a Si arm (light grey) crossing with a poly-Si/TiN (dark grey) arm, as in the FinFET (GC III). The figures are not to scale. 
Table 4.2 $W$ and $L$ dimensions of the GC test structures used for the design

\begin{tabular}{ccc}
\hline \hline & W of lateral $\times$ vertical arm & L of lateral $\times$ vertical arm \\
\hline \hline GC (I) & $100 \mathrm{~nm} \times 100 \mathrm{~nm}$ & $25 \mu \mathrm{m} \times 15 \mu \mathrm{m}$ \\
& $1 \mu \mathrm{m} \times 1 \mu \mathrm{m}$ & $25 \mu \mathrm{m} \times 15 \mu \mathrm{m}$ \\
& & \\
GC (II) & $5 \mu \mathrm{m} \times 5 \mu \mathrm{m}$ & $25 \mu \mathrm{m} \times 15 \mu \mathrm{m}$ \\
& $10 \mu \mathrm{m} \times 10 \mu \mathrm{m}$ & $25 \mu \mathrm{m} \times 15 \mu \mathrm{m}$ \\
& $100 \mathrm{~nm} \times 5 \mu \mathrm{m}$ & $25 \mu \mathrm{m} \times 15 \mu \mathrm{m}$ \\
GC (III) & $1 \mu \mathrm{m} \times 10 \mu \mathrm{m}$ & $25 \mu \mathrm{m} \times 15 \mu \mathrm{m}$ \\
&
\end{tabular}

A commonly used structure to characterize Si-metal contacts is the Cross Bridge Kelvin Resistor (CBKR), as shown in Fig. $4.3[1,4,5]$. The measurement principle is forcing a current $(I)$ between pads 1 and 2 and measure the voltage drop $\left(V_{34}\right)$ between pads 3 and 4. The Kelvin resistance $R_{\mathrm{k}}$ can be found as:

$$
R_{k}=\frac{V_{34}}{I}
$$

To obtain the specific contact resistance $\left(\rho_{c}\right)$, a 2D-Model needs to be applied since there is an overlap $\delta>0$ (see Fig. 4.3). This results in a lateral current flow around the contacts leading to an additional voltage drop, which results in a higher Kelvin resistance [6, 7]. In this model, the measured $R_{\mathrm{k}}$ is then a result of the current through and around the contact. According to the model, $\rho_{c}$ can be calculated using Eq. (4.3):

$$
R_{k}=\frac{\rho_{c}}{A}+\frac{4 R_{s h} \delta^{2}}{3 W_{x} W_{y}}\left[1+\frac{\delta}{2\left(W_{x}-\delta\right)}\right]
$$

where $R_{\text {sh }}$ is the sheet resistance of the overlapping of the underlying Si layer. The other structure parameters are shown in Fig. 4.3.

The CBKR structure was defined in the same way as Si fins (see section 4.1.2). After the cross was defined, gate oxidation was done and TiN and $\alpha$-Si layer were deposited as gate layers. Gate etching was done, leaving a contact hole in the center of the cross, as shown in Fig. 4.3. Afterwards, S/D implantation and activation was done. The lift off mask was defined and the gate oxide was removed to prepare the contact holes for the metallization. After Al-Si deposition, the lift off process was done (for further details see section 4.1.2). The dotted area in Fig. 4.3 indicates the metallic layer. Square contact holes show the region where the actual metal-Si contact is. The dimensions of the structure are also shown in the inset. 


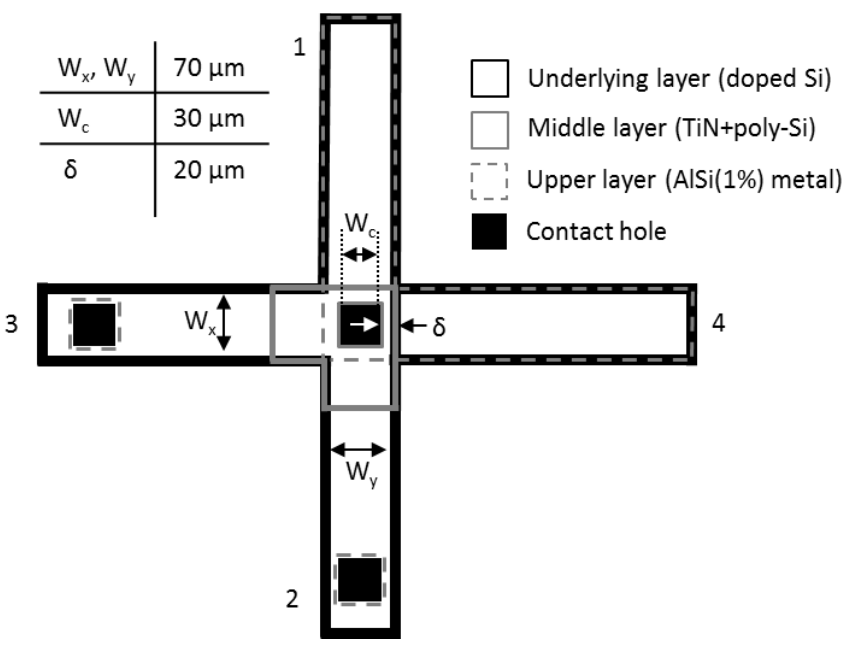

Fig. 4.3 Four terminal CBKR structure with given design parameters.

In addition to resistance measurements, measurement of the gate capacitance is also important for analyzing any additional capacitances, leakage, interface traps, or fixed charges in the gate oxide. For this purpose, capacitors with different areas were designed. Together with dedicated test structures, open and short de-embedding structures were also designed. The designed test structures have a Ground-Signal-Ground (GSG) configuration suitable for RF measurements. Fig. 4.4 shows the planar test structures for C-V measurements as well as its de-embedding pairs. Table 4.3 summarizes the dimensions of the designed test capacitors. As shown in this table, the capacitors include one very wide fin as well as 20-60 parallel narrow fins in the design. The advantage of having parallel fin capacitors is to compare and observe any effect of the edges of the fins.
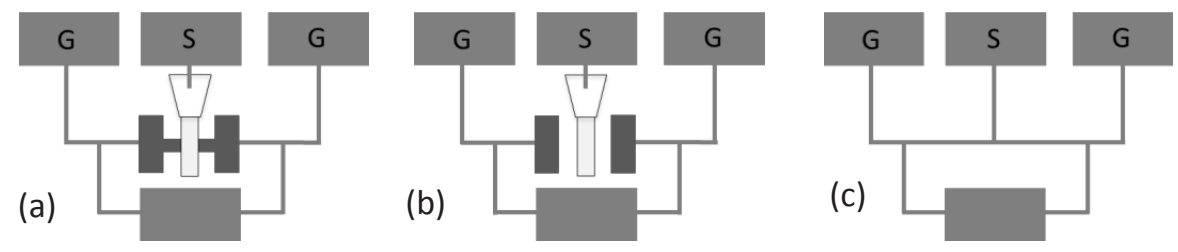

Fig. 4.4 C-V test structures with GSG connections for (a) C-V measurement, (b) open de-embedding and (c) short de-embedding structure. 
Table 4.3 Design parameters of the C-V test structures

\begin{tabular}{cccc}
\hline \hline number of fins & $\boldsymbol{W}_{\boldsymbol{F I N}} \boldsymbol{\&} \boldsymbol{L}_{\text {gate }}$ & $\boldsymbol{W}_{\boldsymbol{F I N}} \boldsymbol{\&} \boldsymbol{L}_{\text {gate }}$ & $\boldsymbol{W}_{\boldsymbol{F I N}} \boldsymbol{\&} \boldsymbol{L}_{\text {gate }}$ \\
\hline \hline 1 & $20 \mu \mathrm{m} \& 20 \mu \mathrm{m}$ & $10 \mu \mathrm{m} \& 20 \mu \mathrm{m}$ & $4 \mu \mathrm{m} \& 20 \mu \mathrm{m}$ \\
20 & $20 \mathrm{~nm} \& 20 \mu \mathrm{m}$ & $30 \mathrm{~nm} \& 20 \mu \mathrm{m}$ & $40 \mathrm{~nm} \& 20 \mu \mathrm{m}$ \\
40 & $20 \mathrm{~nm} \& 20 \mu \mathrm{m}$ & $30 \mathrm{~nm} \& 20 \mu \mathrm{m}$ & $40 \mathrm{~nm} \& 20 \mu \mathrm{m}$ \\
60 & $20 \mathrm{~nm} \& 20 \mu \mathrm{m}$ & $30 \mathrm{~nm} \& 20 \mu \mathrm{m}$ & $40 \mathrm{~nm} \& 20 \mu \mathrm{m}$ \\
\hline \hline
\end{tabular}

\subsubsection{Processing}

As described in section 1.2, (001) oriented SOI wafers are used as a substrate in this work. Initially, the thickness of the Si on oxide was $1.5 \mu \mathrm{m}$. By thermally oxidizing Si and wet etching of the oxide, the thickness of the silicon was decreased to $150 \mathrm{~nm}$. The thickness of the Si layer determines the height of the fin. Si fins as well as S/D regions were patterned with an e-beam lithography (EBL) process. Gate patterning was done by photolithography (PL). The alignment of the devices to the crystal orientation is illustrated in Fig. 4.5. The fin sidewall orientation of the devices is $(1 \overline{1} 0)$ while the surface or crystal plane orientation of the wafer is (001). The crystal orientation of the device is confirmed by TEM analysis in section 4.2.1. To align the EBL patterned features to the PL patterned ones, global alignment crosses as well as write-field alignment crosses were etched through the buried oxide (BOX) layer down to the Si substrate before the device processing. The global alignment crosses are required to find the region of interest for the $\mathrm{EBL}$ process. Once the coordinate system is defined, the system is able to find and align the structures to the write-field alignment crosses. Then, the structures can be written with a relatively good alignment. The process flow is depicted in Fig 4.6.

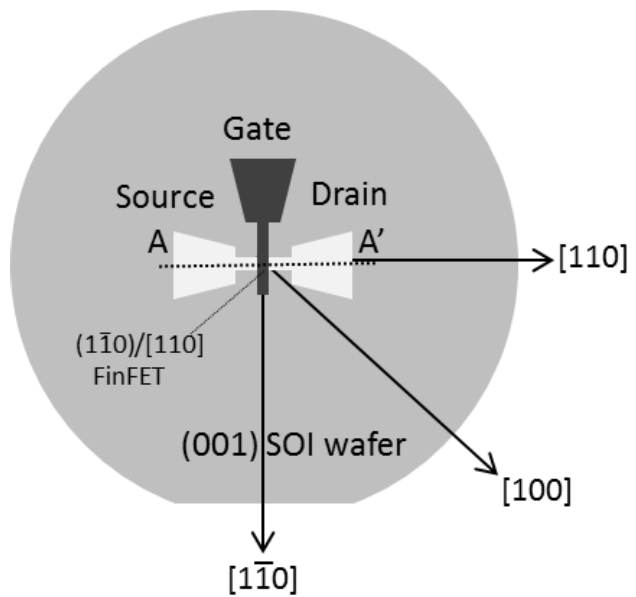

Fig. 4.5 Schematic layout of a $(1 \overline{1} 0) /[110]$ oriented FinFET fabricated on a (001) SOI wafer. The layout is not to scale. For the sake of clarification the FinFET has been magnified drastically. 

(a) Thinning down the Si
(e) Gate oxidation \& gate metallization

(b) Alignment crosses etching (f) Gate etching
(c) $E B L$
(g) S/D implantation

(d) Si fin etching

(h) Contact preparation and metallization

(a)

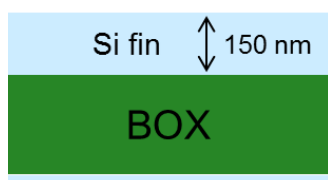

$\mathrm{Si}$ (c)

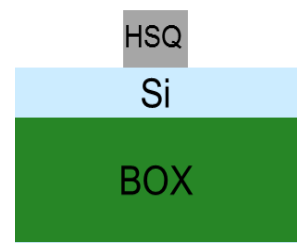

$\mathrm{Si}$

(f)
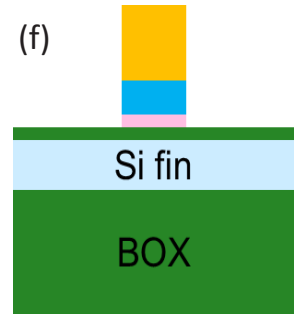

$\mathrm{Si}$ (d)

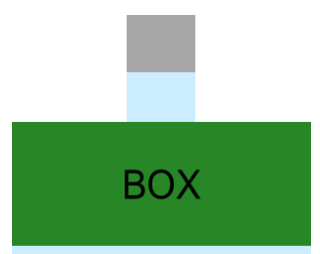

$\mathrm{Si}$

(g)

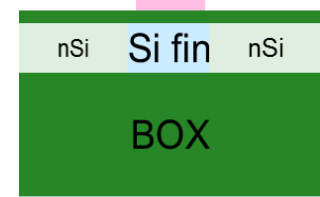

$\mathrm{Si}$ (b)

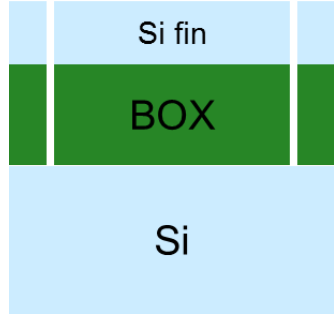

(e)

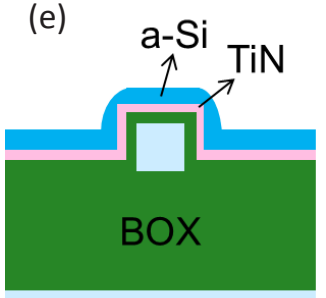

$\mathrm{Si}$

(h)

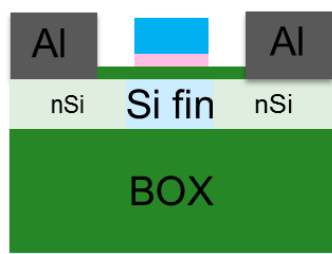

$\mathrm{Si}$ (b) top view

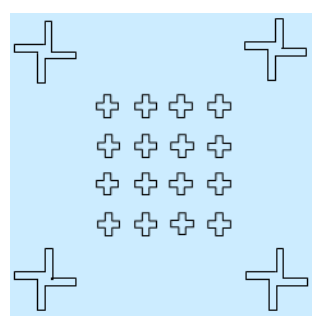

(f)

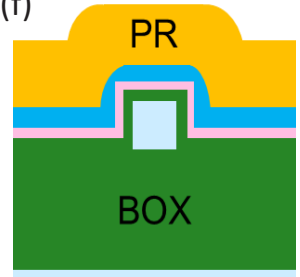

$\mathrm{Si}$

(h)

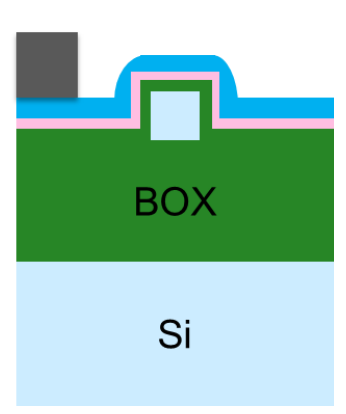

\section{$A-A^{\prime}$ cross section $\quad A-A^{\prime}$ 'cross section}

A-A' cross section

Fig. 4.6 Schematic process flow of the FinFET showing the process steps described in (a)-(h). The A$A^{\prime}$ cross sections are taken in the current flow direction as indicated in Fig. 4.5. 
The processing can be described in the following order:

(a) Thinning down the Si: The SOI layer thickness was initially $1.5 \mu \mathrm{m}$. The Si layer thickness is reduced to $150 \mathrm{~nm}$ by a 3 step process. In step 1, the Si was wet oxidized for 6 hours at $1050^{\circ} \mathrm{C}$ and then the grown oxide layer of $1050 \mathrm{~nm}$ was wet etched in BHF. After this step, the Si layer thickness was measured as $1050 \mathrm{~nm}$ with an ellipsometer. In step 2 and 3 , the same processing was done as described for step 1 . After the $3^{\text {rd }}$ step, the $\mathrm{Si}$ layer thickness was reduced to $150 \mathrm{~nm}$. Ellipsometer measurements showed that this $\mathrm{Si}$ layer has the right thickness and a good uniformity after etching.

(b) Alignment crosses etching: Alignment crosses are needed for alignment in the e-beam lithography (EBL) and photolithography (PL) process. After patterning with PL using a 1.7 $\mu \mathrm{m}$ thick mask, crosses were etched down to the Si substrate using a 2-step etch process. First, $150 \mathrm{~nm}$ Si layer was dry etched in $\mathrm{CHF}_{3}+\mathrm{SF}_{6}+\mathrm{O}_{2}$ plasma. Next, the BOX layer of $1 \mu \mathrm{m}$ was wet etched in BHF. Fig. 4.6 (b) shows the side and top view of the crosses for global and manual alignment of the EBL structures.

(c) EBL and (d) Si fin etching: A negative tone HSQ 1541 resist with $4 \%$ concentration was used in the EBL process. After spinning the resist at a rate of $5000 \mathrm{rpm}$, a $70 \mathrm{~nm}$ thick resist layer was obtained on the Si. After the resist processing the EBL exposure was done with $20 \mathrm{keV}$ beam energy, $0.12 \mathrm{nA}$ beam current, $100-300 \mu \mathrm{C} / \mathrm{cm}^{2}$ area and 1150-1950 $\mathrm{pC} / \mathrm{cm}$ line dose, respectively. The EBL process was performed by manually aligning the EBL mask to the alignment crosses etched before. The auto-focus feature of the EBL system was also used to keep the beam focused to the substrate during the complete patterning process. After exposure, the resist was developed in OPD 4262 for $30 \mathrm{sec}$. The EBL patterned $\mathrm{Si}$ fin structures as well as $\mathrm{S} / \mathrm{D}$ extensions were dry etched in a plasma etcher. RIE was done in $\mathrm{Cl}_{2}(17.5 \mathrm{sccm}), \mathrm{HBr}(25 \mathrm{sccm})$ and $\mathrm{O}_{2}(3 \mathrm{sccm})$ at 10 mTorr with 80 W source power and $350 \mathrm{~W} \mathrm{RF}$ power. $\mathrm{Cl}_{2}$ and $\mathrm{HBr}$ based recipes are known to have high etch rates for $\mathrm{Si}$ and high selectivity to oxide. In this case, we obtained Si:HSQ=10:1 selectivity. Fins and S/D regions were successfully etched in this plasma with a 150 $\mathrm{nm} / \mathrm{min}$ etch rate and high anisotropy. Subsequently, the HSQ was removed in $30 \mathrm{sec}$ using an $\mathrm{HF} 1 \%$ solution.

(e) Gate oxidation \& gate metallization: After patterning the Si fin and S/D regions, a gate oxide layer of $11 \mathrm{~nm}$ was grown by thermal oxidation (measured by ellipsometer). Next, a $6 \mathrm{~nm}$ thick TiN layer was deposited with an in-house atomic layer deposition (ALD) system [8] by controlling the growth with an in-situ spectroscopic ellipsometer (SE). To prevent the oxidation of TiN when exposed to air, a $12 \mathrm{~nm}$ amorphous $\mathrm{Si}(\alpha-\mathrm{Si})$ layer was in-situ deposited without a vacuum break. The deposition of $\alpha$-Si was done in the same reactor 
at $350{ }^{\circ} \mathrm{C}$ by low pressure chemical vapor deposition (LPCVD) at 1 mbar process pressure using trisilane $\left(\mathrm{Si}_{3} \mathrm{H}_{8}\right)$ as the source gas.

(f) Gate etching: After the gate layer was patterned by PL using a $1.7 \mu \mathrm{m}$ thick PR mask, the native oxide was removed in HF solution. And then, the $\alpha$-Si capping layer etching was first done in a $\mathrm{Cl}_{2}$ based plasma etcher with a similar process used for the $\mathrm{Si}$ fin etching. However, due to the anisotropic nature of the parallel plate plasma etcher, the $\alpha$-Si layer could not be completely removed from the fin sidewalls. During the subsequent wet etching of the TiN, the $\alpha$-Si spacer will act as a mask leaving TiN at the sidewall as well. Fig. 4.7 shows a HRSEM picture of the sidewall after $\alpha$-Si etching. A spacer is clearly observable in this picture. Fig. 4.8 shows the obtained I-V curve after finishing the processing of this device. A high gate current of up to $10^{-5} \mathrm{~A}$ is observed due to the short circuit between the S/D regions and the gate, formed by a metal spacer. Therefore, an isotropic etching step must be done to remove the materials from the sidewalls of the $\mathrm{Si}$ fin. Although less anisotropic etching was achieved by changing the process parameters, we were not able to remove the metal layer completely from the sidewalls using the same plasma etching system.

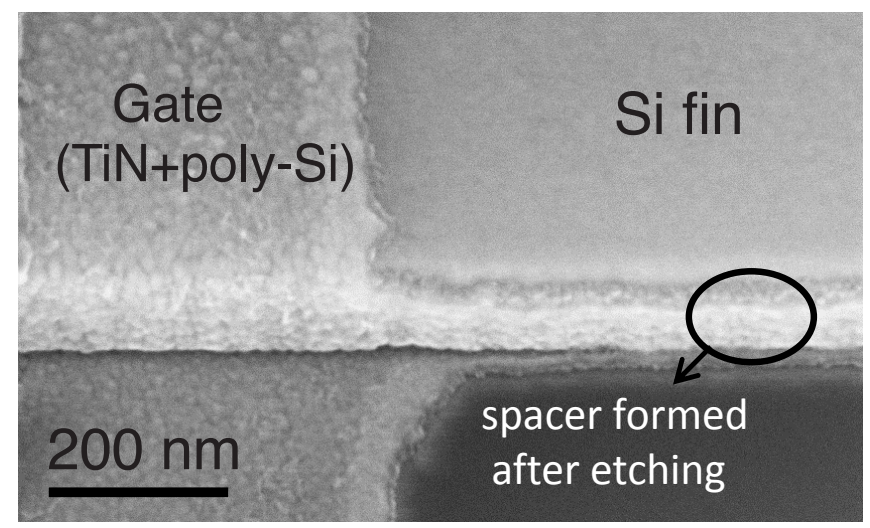

Fig. 4.7 Top view HRSEM picture of the fin sidewall. 


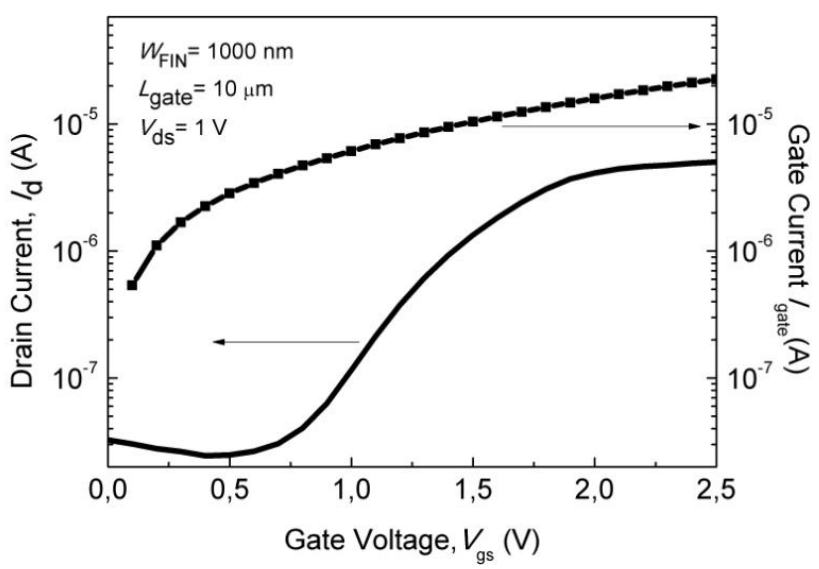

Fig. 4.8 $I_{d}-V_{g s}$ and $I_{\text {gate }}-V_{g s}$ device characteristics with $W_{\mathrm{FIN}}=1000 \mathrm{~nm}$ and $L_{\text {gate }}=10 \mu \mathrm{m}$ at $V_{d s}=1 \mathrm{~V}$. The obtained high gate leakage current is due to the metal spacers.

In contrast to the parallel plate plasma etching systems, barrel reactors are known to provide isotropic etching. Therefore, the $\alpha$-Si was dry etched in $\mathrm{CF}_{4} / \mathrm{O}_{2}$ plasma by using a barrel reactor at the MiPlaza cleanroom, Eindhoven, The Netherlands ${ }^{1}$. High resolution scanning electron microscope (HRSEM) pictures after etching showed that the metallic layer could be completely removed from the sidewalls. After successful etching of the $\alpha$ Si layer, the TiN layer was wet etched using $\mathrm{H}_{2} \mathrm{O}_{2}+\mathrm{NH}_{4} \mathrm{OH}+\mathrm{H}_{2} \mathrm{O}$ solution in a 2 min. etching process. Therefore, a much lower gate current was obtained $\left(\approx 2 \times 10^{-15} \mathrm{~A}\right)$.

(g) S/D implantation: The PR mask used for gate etching was also used for masking the implantation process. The implantation dose parameters were determined by $2 \mathrm{D}$ Athena (Silvaco) process simulations. Aiming to have a doping concentration of $10^{19} \mathrm{~cm}^{-3}$, the S/D regions were implanted with $A s^{+}$with a $1 \times 10^{15} \mathrm{~cm}^{-2}$ dose and at $50 \mathrm{keV}$. The PR was stripped in $\mathrm{O}_{2}$ plasma after implantation. After wafer cleaning, a rapid thermal annealing (RTA) was done in a two-step process. Before reaching the maximum temperature, the wafer was maintained at $600^{\circ} \mathrm{C}$ for $10 \mathrm{sec}$ and then the RTA at $900{ }^{\circ} \mathrm{C}$ for $30 \mathrm{sec}$ was done to activate the dopants. The main reason for this two-step process is to attune the metal stack to a high temperature treatment. Due to high temperature annealing, the $\alpha$-Si crystallizes. Therefore, it is stated as poly-Si in the rest of this thesis.

It is important to note that the TiN induced strain in FinFETs as discussed in Chapter 2 is not expected in this device structure. First, ALD TiN is known to induce less strain $(\approx 50 \%$ less) than physical vapor deposited TiN and it is close to zero for TiN films with a layer thickness below $10 \mathrm{~nm}$ [9]. Second, the RTA temperature is $900{ }^{\circ} \mathrm{C}$ whereas NXP-TSMC FinFET devices were exposed to $1100{ }^{\circ} \mathrm{C}$. This means that the stress due to the thermal

\footnotetext{
${ }^{1}$ This part of the processing was performed by R.A.M. Wolters, with courtesy.
} 
expansion coefficient difference between that of the TiN metal gate and the Si will be less. Third, we used an $11 \mathrm{~nm}$ thick oxide layer, which is almost 5 times thicker than that of NXP-TSMC FinFETs. This relatively thick oxide layer under the TiN would result in less strain in Si. Therefore, our FinFETs will have a different strain distribution than that of the strained FinFETs discussed in Chapter 2. We expect that the amount of strain is low due to the reasons mentioned above.

(h) Contact preparation and metallization: The metal patterns were defined by PL using a shadow mask. Prior to metal deposition (gate) oxide on the contact holes was removed by $1 \% \mathrm{HF}$ etching. Metal deposition was done by sputtering from an Al-Si (1\%) target to obtain a $250 \mathrm{~nm}$ thick metal layer. The patterning was finalized by a lift-off process in acetone. As a last step, to reduce the amount of interface traps, $100 \% \mathrm{H}_{2}$ annealing at 10 mbar was done at $420{ }^{\circ} \mathrm{C}$ for $20 \mathrm{~min}$. Fig. 4.9 shows an optical micrograph top view of a fabricated device structure.

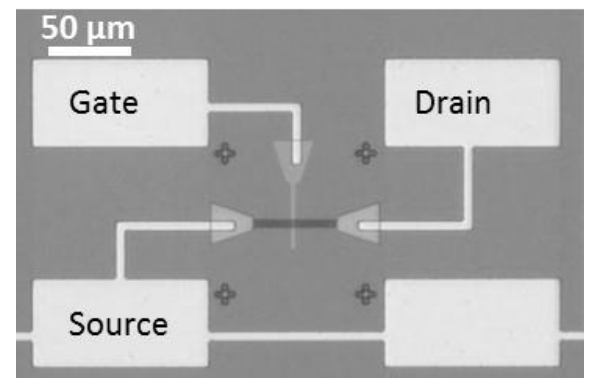

Fig. 4.9 Optical micrograph of a typical FinFET. Note the write field alignment marks are needed for EBL.

\subsection{Physical and electrical characterization of FinFETs}

Analyses of the fabricated FinFETs were done by physical and electrical characterization techniques. Focused ion beam (FIB) as well as transmission electron microscope (TEM) were used to study cross sections of the fabricated 3D device structure. The electrical characterization of the FinFETs was done at room temperature by using a SüSS Microtech probe station equipped with a Keithley 4200 semiconductor characterization system.

\subsubsection{FIB/TEM analysis}

To take a cross section picture of the fabricated FinFETs, the sample was prepared with a FIB tool by cutting through the Si fin in the channel region. After preparation, a TEM picture of the FinFET was taken. Fig. 4.10 (a) shows a 5-fin structure with a $100 \mathrm{~nm}$ fin width. A closer view of one fin is shown in Fig. 4.10 (b). In addition, a Si fin with $20 \mathrm{~nm}$ 
designed width is presented in Fig. 4.10 (c). Fig. 4.10 (b) shows that the real fin width is very close to the designed width of $100 \mathrm{~nm}$. Fig. 4.10 (c), on the other hand, shows that the actual fin width is changing along the fin height. The fin width is measured to be $\approx 14$ $\mathrm{nm}$ for the thickest region. Therefore, the actual fin width is smaller than the designed width of $20 \mathrm{~nm}$. For convenience sake in this work the designed fin width will be used as a reference unless stated otherwise.

The stack of $\mathrm{SiO}_{2}+\mathrm{TiN}+$ poly-Si layer is observed around the fin. The cross section picture indicates that the channel is well defined and that the poly-Si/TiN layers have good step coverage. The layers on the sidewalls are clearly observable. The information from the top of the fin was lost due to sample preparation. As will be shown in chapter 5 , this region is also clearly observable when there is a PZT layer on top of the fin.
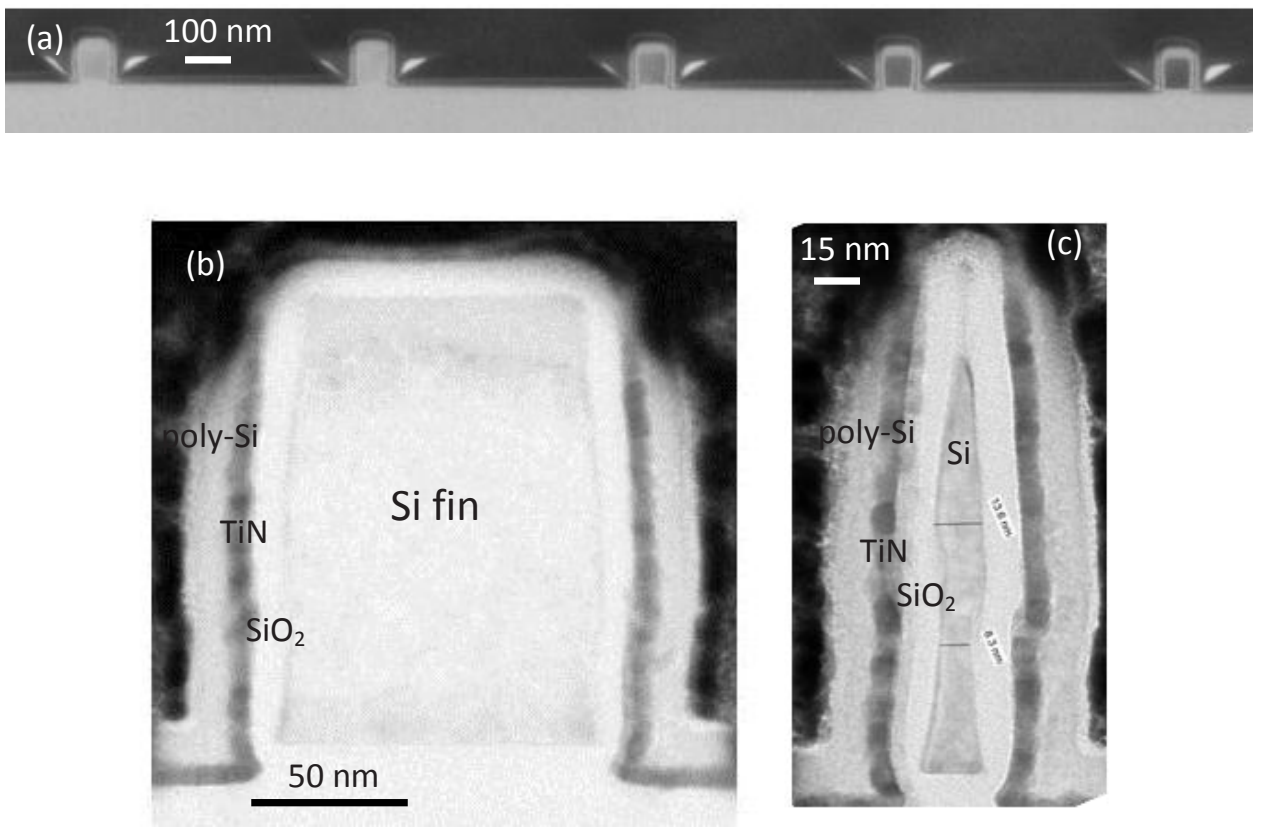

Fig. 4.10 A TEM cross section picture of (a) a 5-fin FinFET with $100 \mathrm{~nm}$ fin width, magnified view of a (b) $100 \mathrm{~nm}$ and (c) $20 \mathrm{~nm}$ wide designed single fin.

\subsubsection{Resistance measurements}

Resistance measurements give a quick understanding of the basic properties of the thin films. In this section, the GCs presented in Fig. 4.2 and the CBKR presented in Fig. 4.3 are used. These analyses give a quick assessment of the process control. They are important to know before the characterization of the actual FinFETs. 
To calculate the doping concentration in the S/D regions and resistivity of the deposited gate stack, sheet resistance measurements were done using the GC I and GC II structures in Fig. 4.2. The sheet resistance of GC I with $1 \mu \mathrm{m} \times 1 \mu \mathrm{m}$ wide arms was determined to be $204 \Omega / \square$. Using this value, the resistivity $(\rho)$ of the doped S/D contact region can be calculated according to [1]:

$$
\rho=R_{s h} \times t
$$

where $t$ is the thickness of the film (i.e. $150 \mathrm{~nm}$ ). The resistivity of the doped $\mathrm{Si}$ is calculated being $\approx 3.1 \times 10^{-3} \Omega-\mathrm{cm}$ using Eq. (4.4). The doping concentration corresponding to this resistivity is around $10^{19} \mathrm{~cm}^{-3}[10]$. This value is in agreement with the projected doping concentration mentioned in section 4.1.2.

The GC I test structure with $100 \mathrm{~nm}$ wide arms showed a much higher resistivity than that of $1 \mu \mathrm{m}$ wide arm. The possible reason for this discrepancy is the incompatibility of the GC method for nanometer sized structures where edge effects become dominant and affects the resistance measurements. Another method can be used to obtain the actual resistivity of such narrow structures, such as the transfer length method [1]. The sheet resistance obtained from the GC II with $5 \mu \mathrm{m} \times 5 \mu \mathrm{m}$ and with $10 \mu \mathrm{m} \times 10 \mu \mathrm{m}$ wide arms is $264 \Omega / \square$. Using Eq. (4.4), the resistivity of the TiN is calculated to be $\approx 160 \mu \Omega-\mathrm{cm}$. This value is in agreement with the value obtained from the different structures using the same setup [11]. The sheet resistance of the GC III was measured as $\approx 3 \times 10^{5} \Omega / \square$. This high value is an indication that there are no active dopants in the Si channel, as expected from TCAD simulations.

To obtain the specific contact resistance, the CBKR structure shown in Fig. 4.3 was measured. The resistance is measured as $R_{k}=35 \Omega$ by applying a current between pad 1 and 3 and measuring voltage in pad 3 and $4 . \rho_{c}$ can be calculated using Eq. (4.3) and inserting the known parameters. The calculated $\rho_{c}$ is in the order of $10^{-4} \Omega-\mathrm{cm}^{2}$. This value is in the same order of magnitude as mentioned in previous reports [12].

\subsubsection{I-V characterization and analysis}

FinFETs with different $W_{\text {FIN }}$ and $L_{\text {gate }}$ were measured and analyzed. Most of the single fin devices, especially with narrow fins, were not functional. 5-fin devices, on the other hand, showed better characteristics. All presented characteristics belong to devices with 5-fins unless stated otherwise. Fig. 4.11 shows the $I_{d}-V_{d s}$ curve of the eight different fabricated FinFETs. 

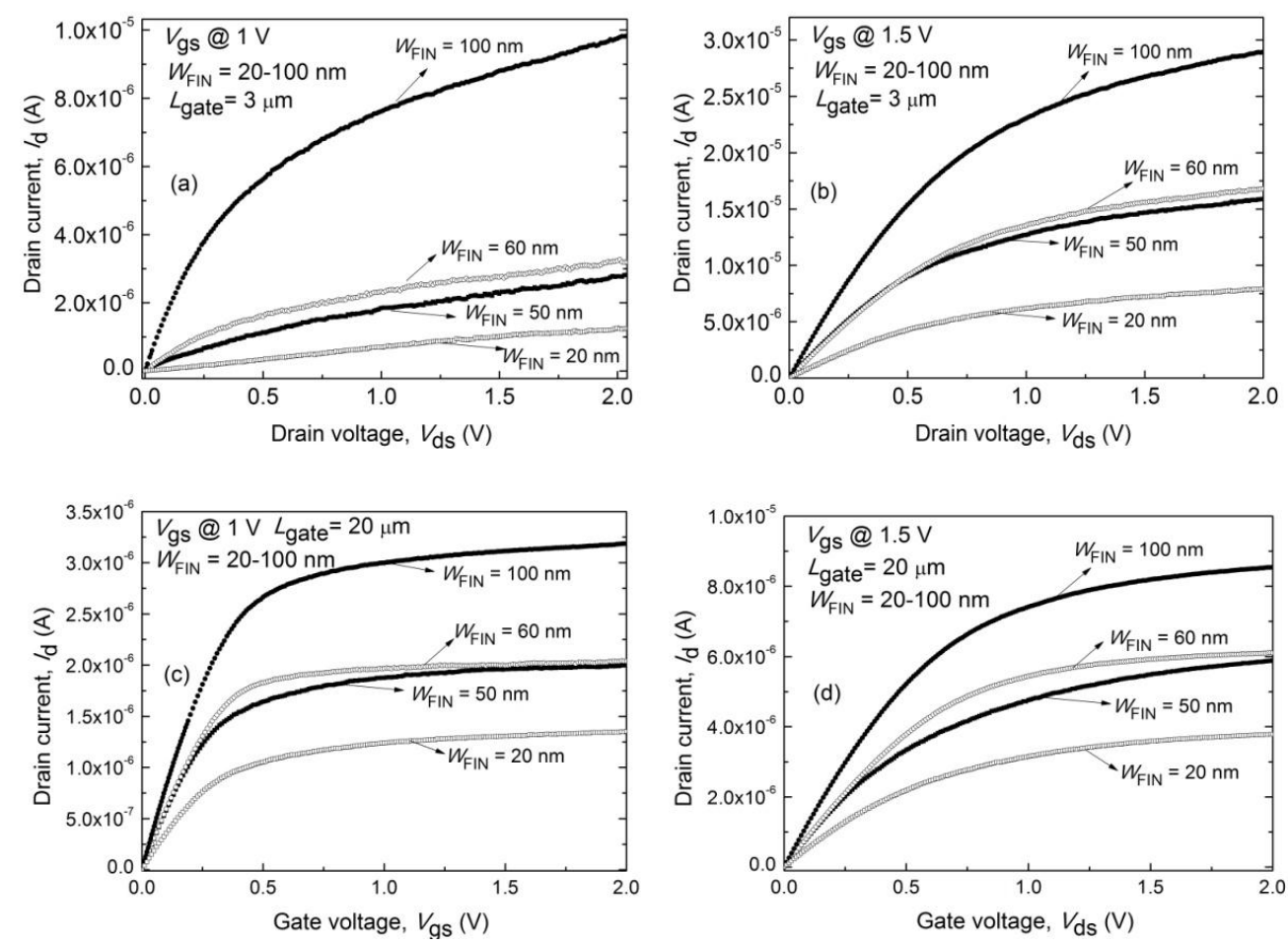

Fig. $4.11 I_{d}-V_{d s}$ characteristics of devices with $W_{\text {FIN }}=20 \mathrm{~nm}-100 \mathrm{~nm}$ and (a) $L_{\text {gate }}=3 \mu \mathrm{m}, V_{g s}=1 \mathrm{~V}$, (b) $L_{\text {gate }}=3 \mu \mathrm{m}, V_{g s}=1.5 \mathrm{~V}$, (c) $L_{\text {gate }}=20 \mu \mathrm{m}, V_{g S}=1 \mathrm{~V}$, (d) $L_{\text {gate }}=20 \mu \mathrm{m}, V_{g s}=1.5 \mathrm{~V}$. The measurements were performed at $300 \mathrm{~K}$.

In typical $I_{d}-V_{d s}$ curves, there are three regimes of operation, namely linear, non-linear and saturation regions $[10,13]$. In the linear region, where $V_{\mathrm{ds}}$ is less than $V_{\mathrm{gs}}$, the drain current of long channel FETs can be expressed as follows:

$$
I_{d}=\mu_{n} C_{o x} \frac{W}{L_{\text {gate }}}\left[\left(V_{g s}-V_{t h}\right) V_{d s}-\frac{1}{2} V_{d s}^{2}\right]
$$

whereas in the saturation region the drain current can be expressed as [13]:

$$
I_{d}=\mu_{n} C_{o x} \frac{W}{L_{\text {gate }}}\left(V_{g s}-V_{t h}\right)^{2}
$$

where $\mu_{n}$ is the electron mobility, $C_{\mathrm{ox}}$ is the gate oxide capacitance per unit area, $W$ is the channel width ( $=\mathrm{N}_{\mathrm{FIN}} \times\left(2 \mathrm{H}_{\mathrm{FIN}}+W_{\mathrm{FIN}}\right), \mathrm{N}_{\mathrm{FIN}}$ is the number of active fins), $L_{\text {gate }}$ is the gate length, $V_{\text {th }}$ is the threshold voltage; the gate-source voltage at which the inversion layer is formed at the interface between the gate oxide and the Si. 
Fig. 4.11 shows the $I_{d}-V_{d s}$ curves for short channel ( $3 \mu \mathrm{m}$ gate length) and long channel devices (20 $\mu \mathrm{m}$ gate length). The latter was chosen to reduce short channel effects and to avoid a high S/D resistance. It can be concluded from the figure that the $I_{d}$ does not scale with the channel width as described by Eq. (4.5b). In fact, $I_{d}$ generally increases more sharply with $W$ and this could be due to several effects.

First, if the fin width decreases, the resistance of the S/D extensions will become dominant, especially for the short gate length. This results in a decrease in the drain current, especially in the linear region above threshold. This phenomenon can be observed when Fig. 4.11 (a) and (c) are compared for narrow fins. In addition, irregular fin width results a smaller average fin width over the fin height (Fig. 4.10), which causes nonlinearity. The drain current scaling is more non-ideal for devices with $3 \mu \mathrm{m}$ gate lengths compared to devices with $20 \mu \mathrm{m}$ gate lengths.

Second, a change in the effective channel length can result in stronger than expected scaling of $I_{d}$. Since the doping in the channel region is very low $\left(\approx 10^{15} \mathrm{~cm}^{-3}\right)$, as confirmed in our GC III structures, the depletion layer laterally extends further in the channel region for a wide fin than for a narrow fin device because of improved electrostatic gate control for the latter. This is observed in the saturation region in which the "Early effect" [10] seems to be stronger for a wide fin (Fig. 4.11 (a) and (b)). However, when the channel length is longer, this effect is less important (Fig. 4.11 (c) and (d)).

Third, one or more defective fins and/or line edge roughness (LER) could be the reason for this strong dependency. The LER effect has been shown to be the main source of variability in FinFET devices [14, 15].

Fourth, non-uniform doping in the S/D extensions regions can result in high $I_{\text {on }}$ variability. This effect is more serious for narrow fins and short gate lengths [16]. It could be an indication of less strong scaling observed in our long channel devices in Fig. 4.11 (c) and (d).

The FinFET is in a weak-inversion or depletion mode in the sub-threshold region and the drain current is dominated by diffusion. This region of operation is important to study since this region characterizes how sharply the current drops with the gate bias. This region is important for low power applications. The drain current of a long channel fully depleted FinFET operated at gate voltages below threshold is [13]:

$$
I_{d}=\frac{N_{F I N} H_{F I N} \mu_{n}}{L_{g a t e} \beta} q n_{i} W_{F I N} \exp \left(\beta \varphi_{s}\right)\left[1-\exp \left(-\beta V_{d s}\right)\right]
$$

where $\beta=\mathrm{q} / \mathrm{kT}, \mu_{n}$ is electron mobility, $n_{i}$ is intrinsic carrier concentration, and $\varphi_{s}$ is the surface potential. 
For a fully depleted FinFET the following relation can be obtained:

$$
V_{g s}-V_{f b}=\frac{Q_{i t}}{C_{o x}}+\varphi_{s}=\left(\frac{C_{i t}+C_{o x}}{C_{o x}}\right) \cdot \varphi_{s},
$$

where $Q_{\mathrm{it}}$ is the interfacial trapped charge and $V_{\mathrm{fb}}$ is the work function difference between intrinsic silicon and the gate metal $\left(V_{\mathrm{fb}}=0 \mathrm{~V}\right.$ for a mid-gap metal gate).

The relative change of $V_{g s}$ caused by $\varphi_{s}$ can be found using Eq. (4.7):

$$
\frac{d V_{g s}}{d \varphi_{s}}=\frac{C_{o x}+C_{i t}}{C_{o x}} \equiv \mathrm{n} .
$$

Using Eq. (4.8), Eq. (4.6) can be rewritten as

$$
I_{d}=I_{d 1} \exp \left(\beta\left(V_{g s}-V_{f b}\right) / \mathrm{n}\right)\left[1-\exp \left(-\beta V_{d s}\right)\right],
$$

where $I_{d 1}$ depends on temperature, device dimensions and $\mathrm{n}$ is the ideality factor. In an ideal FinFET with a negligible amount of interfacial traps, $n=1$.

For $V_{d s} \gg k T / q$, Eq. (4.9) can be simplified as:

$$
I_{d}=I_{d 1} \exp \left(\beta\left(V_{g s}-V_{f b}\right) / \mathrm{n}\right) .
$$

Hence, the sub-threshold current in which $\log \left(I_{d}\right)$ is plotted vs. $V_{g S}$, has a slope of $q /[\ln (10) \cdot \mathrm{n} k T]$. The so-called sub-threshold swing (SS) is defined as the inverse of that value, $\ln (10) \cdot \mathrm{n} k T / q$ [10], corresponding to approximately $60 \mathrm{mV} / \mathrm{dec}$ at room temperature for an ideal FinFET (without any traps).

In Fig. 4.12, experimental $I_{d}-V_{g s}$ curves of FinFETs with a different $W_{\text {FIN }}$ are shown for $V_{d s}=25 \mathrm{mV}$ (a) and $V_{d s}=1 \mathrm{~V}$ (b). These curves show a high on- to off-current ratio $I_{\text {on }} / I_{\text {off }}\left(\approx 10^{8}\right)$ and a $S S$ value of $76 \mathrm{mV} / \mathrm{dec}$. 

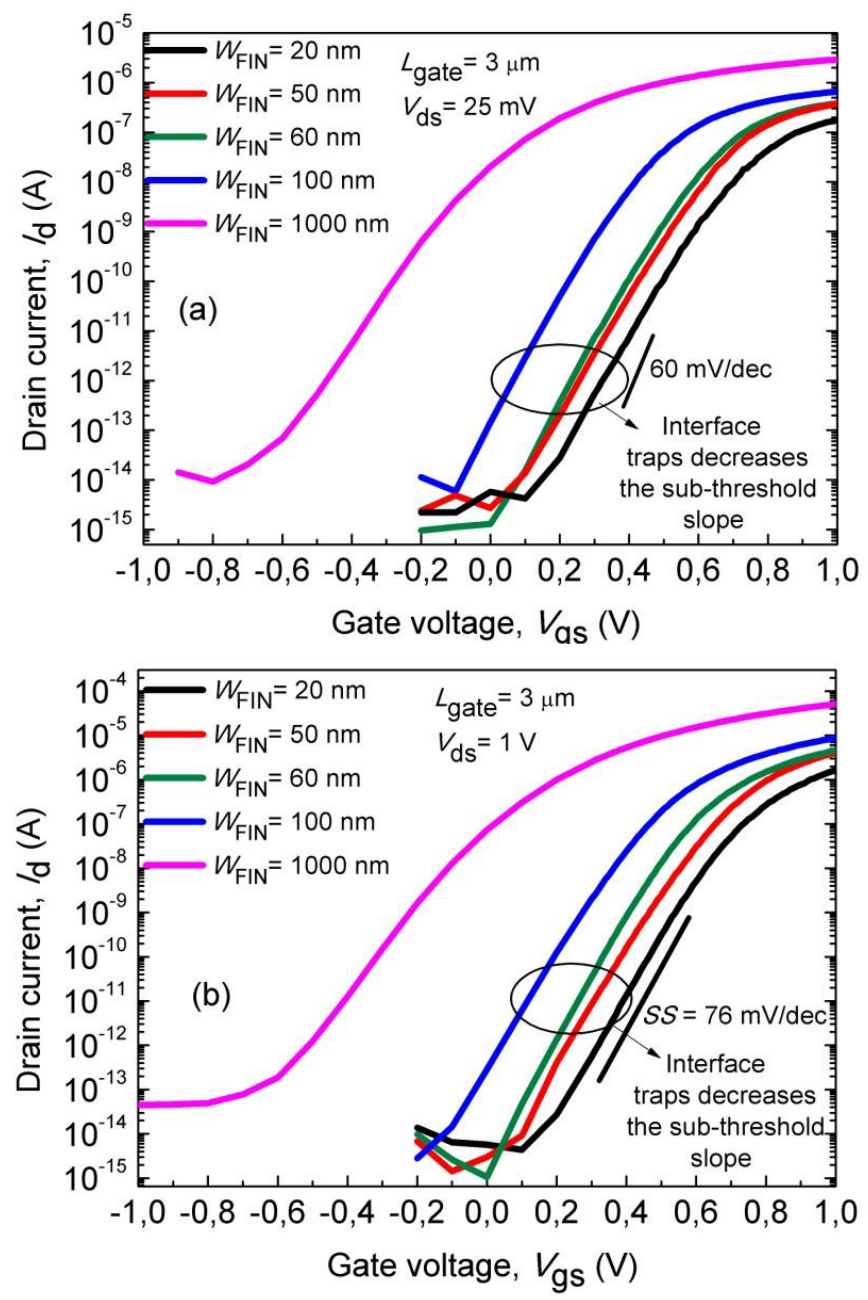

Fig. $4.12 I_{d}-V_{g s}$ characteristics of devices with $L_{\text {gate }}=3 \mu \mathrm{m}$ and $W_{\text {FIN }}=20 \mathrm{~nm}-100 \mathrm{~nm}$, at (a) $V_{d s}=25$ $\mathrm{mV}$ and (b) $V_{d s}=1 \mathrm{~V}$.

As discussed before, the drain current $I_{d}$ increases stronger than expected with the channel width $(W)$. In addition, we observe a short-channel effect for $W_{\mathrm{FIN}}=1000 \mathrm{~nm}$, which is due to less electrostatic gate control, resulting in an increased SS. This effect is related to the so-called drain-induced barrier lowering, or in short DIBL [10], as discussed later.

Another important observation is an increase of the threshold voltage when the fin width decreases. The proportionality of the sub-threshold current to the Si thickness is a manifestation of volume inversion [13]. In the ultra-thin body, there is a limited amount of inversion charge [17] and this is observed as a decrease in the sub-threshold current. 
To compare the performance, $I_{d}-V_{g s}$ characteristics of NXP and our FinFET devices measured at $V_{d s}=1 \mathrm{~V}$ are plotted in Fig. 4.13.

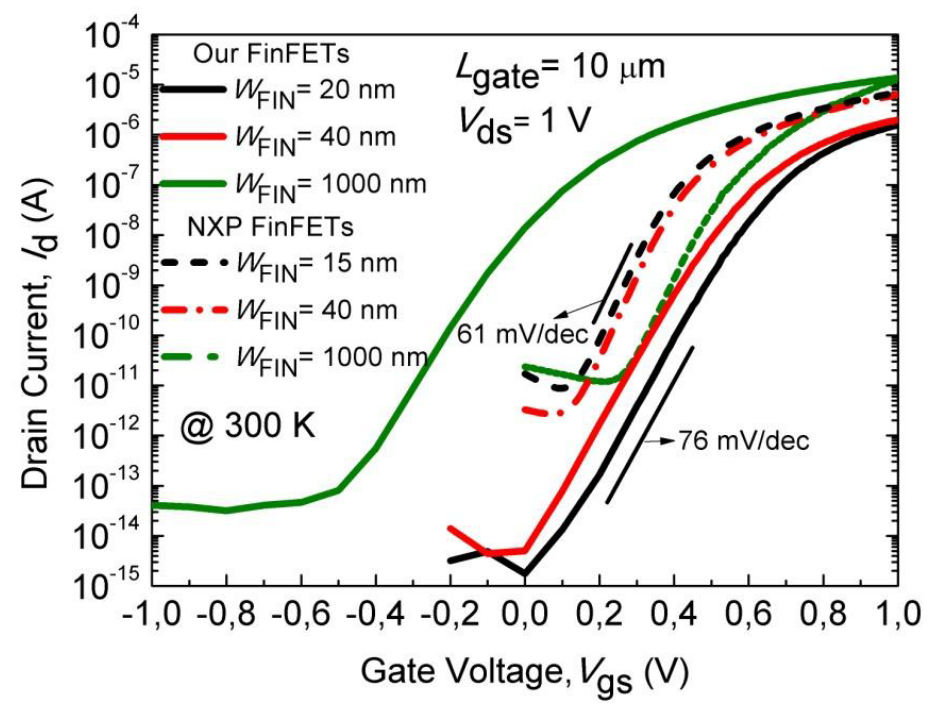

Fig. $4.13 I_{d}-V_{g s}$ characteristics of NXP and our FinFET devices with 5 -fins, $L_{\text {gate }}=10 \mu \mathrm{m}$ and $W_{\text {FIN }}=20$ $\mathrm{nm}, 40 \mathrm{~nm}$ and $1000 \mathrm{~nm}$ at $V_{d s}=1 \mathrm{~V}$. The measurements were performed at $300 \mathrm{~K}$.

Due to shorter S/D extensions in NXP FinFETs $\left(L_{\mathrm{sd}}=90 \mathrm{~nm}\right)$ compared to our FinFETs $\left(L_{s d}=15-23 \mu \mathrm{m}\right), I_{\text {on }}$ is around three times higher for NXP FinFETs for narrow fin devices. Furthermore, $I_{\text {off }}$ is higher and $S S$ is lower for NXP FinFETs $\left(\approx 10^{-11} \mathrm{~A}\right.$ and $\left.\approx 61 \mathrm{mV} / \mathrm{dec}\right)$ than UT FinFETs $\left(\approx 10^{-14} \mathrm{~A}\right.$ and $\approx 76 \mathrm{mV} / \mathrm{dec}$ ). The lower $S S$ is due to less interface states. To summarize, our devices perform well as evidenced by this comparison.

The amount of active interface states in our devices can be calculated using the SS value. The amount of increase in $Q_{\text {it }}$ would increase the $n$ value in Eq. (4.8). The value $n$ can be obtained for UT FinFETs by dividing the $S S$ to the ideal $S S, n=1.27$. Therefore, $C_{i t} / C_{o x}=0.27$. As will be shown later in section $4.2 .4, C_{0 x}=3.1 \times 10^{-7} \mathrm{~F} / \mathrm{cm}^{2}$. Therefore, $C_{\mathrm{it}}=8.37 \times 10^{-8} \mathrm{~F} / \mathrm{cm}^{2}$. Since for the interface trap density holds $D_{\mathrm{it}}=\mathrm{C}_{\mathrm{it}} / q^{2}[1], D_{\mathrm{it}}$ can be calculated as $5.23 \times 10^{11}$ $1 / \mathrm{eV}-\mathrm{cm}^{2}$.

Drain-induced-barrier lowering (DIBL) is another performance degrading mechanism resulting in a shift of the threshold voltage at high drain voltages and consequently high $I_{o f f}$. This effect strongly depends on the channel length. In principle a higher body doping and/or an improved gate control through a narrow fin width is required to reduce this effect by preventing the expansion of drain depletion into the channel. However, high doping results in lower mobility, higher junction capacitance and increased junction leakage [18]. For our FinFETs, the DIBL is shown in $I_{d}-V_{g s}$ curve at different $V_{d s}$ in Fig. 
4.14. DIBL is defined as the decrease in threshold voltage when the drain voltage is increased from $25 \mathrm{mV}$ to $2 \mathrm{~V}$. DIBL is calculated as $93 \mathrm{mV} / \mathrm{V}$, using the following relation:

$$
\operatorname{DIBL}(\mathrm{mV} / \mathrm{V})=\frac{V_{t h 1}-V_{t h 2}}{V_{d s 1}-V_{d s 2}}
$$

Although our devices have a low channel doping, they do not suffer from strong DIBL. This is because our device lengths are in the micrometer range and we use narrow fins. The calculated DIBL value is comparable to the values obtained from simulations by Lee et al. using similar device structures [19].

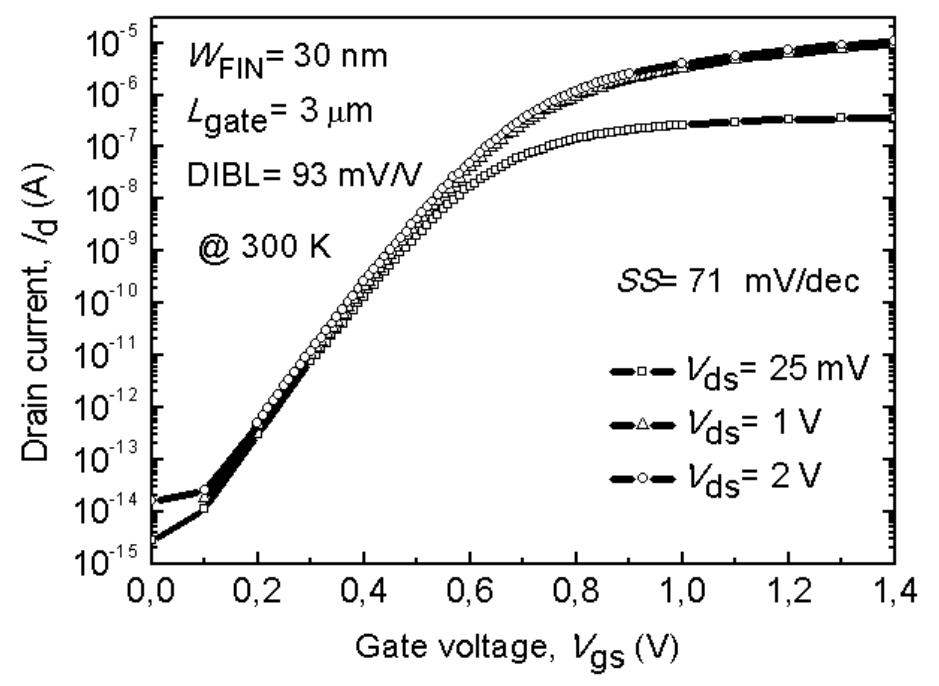

Fig. $4.14 I_{d}-V_{g s}$ curves of devices with $L_{\text {gate }}=3 \mu \mathrm{m}$ and $W_{\text {FIN }}=30 \mathrm{~nm}$, at $V_{d s}=25 \mathrm{mV}, 1 \mathrm{~V}$ and $2 \mathrm{~V}$. The measurements were performed at $300 \mathrm{~K}$.

As mentioned before, an increase in the channel length decreases the sub-threshold leakage by suppressing the short channel effects (SCE). However, it also increases the channel resistance, thereby decreasing $I_{o n}$. $I_{\text {on }}$ and $I_{\text {off }}$ gets smaller for longer gate lengths because of lower leakage and higher channel resistance.

Fig. 4.15 shows $I_{d}-V_{g s}$ curves obtained from devices with various fin widths and gate lengths at $V_{d s}=1 \mathrm{~V}$. The $I_{o f f}$ and $I_{o n}$ scaling with gate length stated before is only observed in Fig. 4.15 (d) for the $W_{\text {FIN }}=1 \mu \mathrm{m}$ device. Figs. 4.15 (a)-(c) show that $I_{\text {off }}$ and $I_{\text {on }}$ show no clear dependency on the gate length for narrow fins. As mentioned in section 4.2 .3 , the cause of this scaling deficiency is possibly caused by defective fins, LER, $S / D$ doping non-uniformity. Furthermore, positive fixed charges at the interface (or in the gate oxide) and interface states (traps) could give a channel length dependence of this effect. Stronger than expected gate length scaling of the drain current holds especially for devices having a narrow fin width and long gate length. This dependence could be due to 
the etching process. While etching the fins, surface imperfections could have been created, which would be more important for narrow fins and long gate lengths. The TEM picture especially for the $20 \mathrm{~nm}$ specified fin width in Fig. 4.10 clearly shows that the actual fin width is smaller than the specified width and also varies over the fin height. The latter also reduces drastically in particular on top of the fin. This could cause an increase of the threshold voltage or the actual average $W_{\text {FIN }}$ drops drastically. As a result, the subthreshold current drops (see Eq. (4.6)). This irregular fin sidewall pattern could also vary across the gate length. Further, other non-idealities such as fixed charge or additional traps could also have been formed by the etching process. Since both sub-threshold and up-threshold region are affected, more than one of these non-idealities could be present.
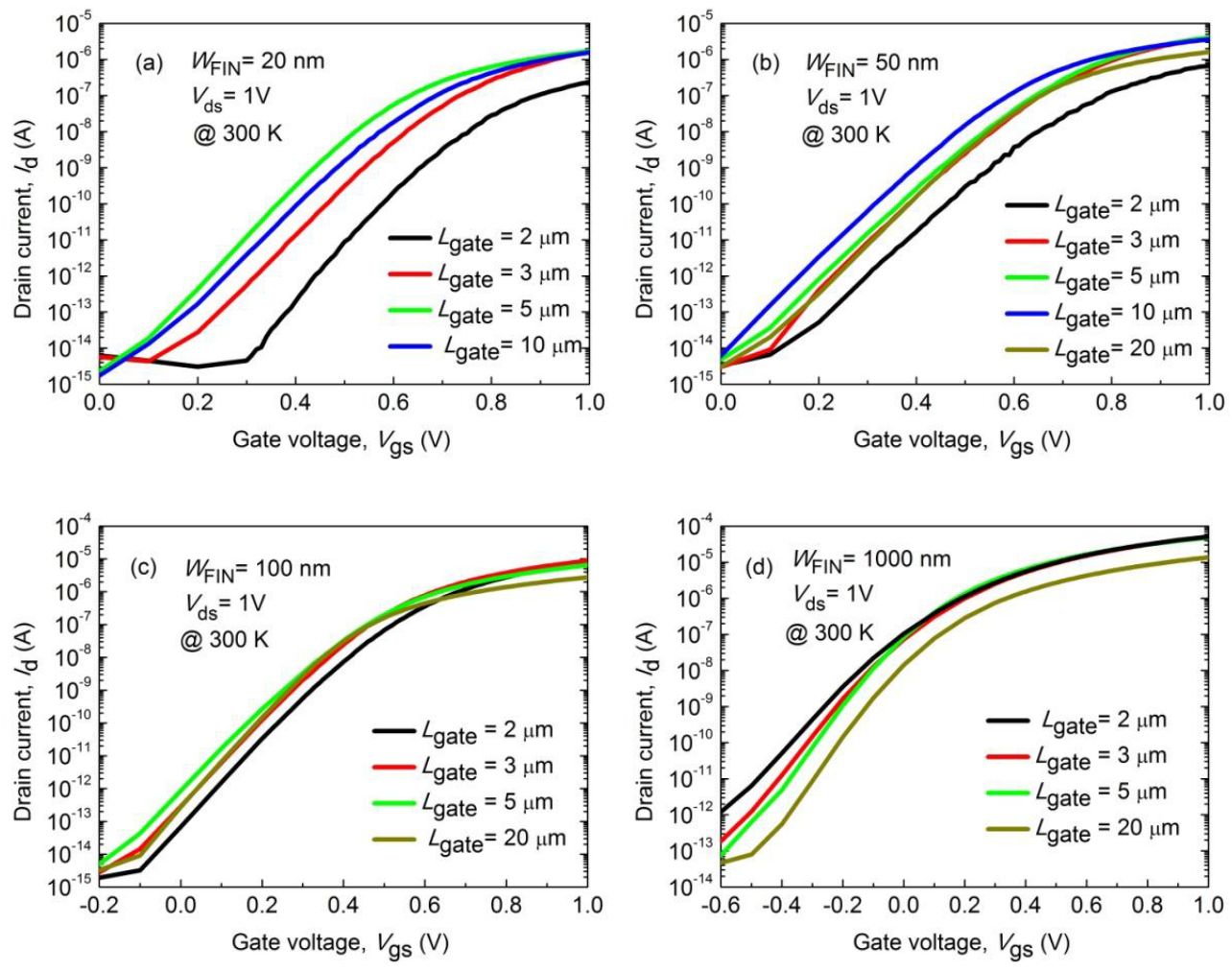

Fig. $4.15 I_{d}-V_{g s}$ device characteristics with $L_{\text {gate }}=2-20 \mu \mathrm{m}$ at $V_{d s}=1 \mathrm{~V}$ for a) $W_{\mathrm{FIN}}=20 \mathrm{~nm}$, b) $W_{\mathrm{FIN}}=$ $50 \mathrm{~nm}, \mathrm{c}) W_{\mathrm{FIN}}=100 \mathrm{~nm}$, and d) $W_{\mathrm{FIN}}=1000 \mathrm{~nm}$. The measurements were performed at $300 \mathrm{~K}$. 


\subsubsection{Mobility calculation}

Mobility extraction of the FinFET is not very straightforward due to some complications such as the body doping effect and different turn on voltages at the top corners and the body of the fin. In this work, the body doping is negligible $\left(\approx 10^{15} \mathrm{~cm}^{-3}\right)$ and hence the structure does not suffer from different turn on voltages. As a result, there is no "kink" in the presented sub-threshold curves.

The mobility of the measured (long channel) FinFETs can be calculated using the following drift and diffusion current relation:

$$
I_{d}=\frac{W \mu_{e f f} Q_{n} V_{d s}}{L_{g a t e}}-\frac{N_{F I N} H_{F I N} \mu_{e f f} k T}{q} \frac{d Q_{n}}{d x}
$$

where $Q_{n}$ is the mobile charge density $\left(C / \mathrm{cm}^{2}\right), \mu_{\text {eff }}$ is the effective mobility generally measured at low $V_{d s}$ (hence low lateral electric field). At low $V_{d s}$ (25-100 mV) and above threshold voltage, the carriers in a uniformly doped channel are uniformly distributed. Therefore, the diffusive second term in Eq. (4.12) can be ignored. To obtain an $\mu_{e f f}$ expression, the mobile charge density needs to be determined. One way to do so is to approximate it by:

$$
Q_{n}=C_{o x}\left(V_{g s}-V_{t h}\right)
$$

Although it is an easy way to determine $Q_{n}$, the charge density is not exactly given by this expression. However, it is a good approach to obtain the mobility for above threshold and would give representative numbers.

Inserting Eq. (4.13) in Eq. (4.12) and ignoring the diffusive second term, $\mu_{\text {eff }}$ can be expressed as:

$$
\mu_{e f f}=\frac{I_{d} L_{g a t e}}{W C_{o x}\left(V_{g s}-V_{t h}\right) V_{d s}}
$$

$C_{o x}$ is the oxide capacitance per unit area (specific oxide capacitance) and can be calculated using the following relation:

$$
C_{o x}=\frac{C_{o x^{\prime}}}{A}=\frac{\varepsilon_{0} \varepsilon_{o x}}{t_{o x}}
$$

where $\varepsilon_{0} \varepsilon_{o x}=3.45 \times 10^{-11} \mathrm{~F} / \mathrm{m}$, and $t_{o x}=11 \mathrm{~nm}, A=N_{\mathrm{FIN}} \times\left[\left\{\left(2 \times H_{\mathrm{FIN}}\right)+W_{\mathrm{FIN}}\right\} \times L_{\text {gate }}\right]$. Therefore, the oxide capacitance per unit area for these devices was calculated as $3.1 \times 10^{-}$ ${ }^{7} \mathrm{~F} / \mathrm{cm}^{2}$. This value is in good agreement with the measured capacitance values as shown in section 4.2.5. 
The $I_{d}-V_{g s}$ curves (at $V_{d s}=25 \mathrm{mV}$ ) plotted in Fig. 4.16 are used for mobility calculations in Fig. 4.17. Before starting to analyze the mobility graphs, it is wise to first have a look at the behavior of the $I_{d}-V_{g s}$ curves in linear scale. For an ideal device, a linear relationship between drain current and gate voltage is expected; otherwise Eq. (4.12) cannot be used. Fig. 4.16 shows that the curves deviate from linear behavior for $20 \mathrm{~nm}$ and $50 \mathrm{~nm}$ wide devices. The deviation starts at about $0.8 \mathrm{~V}$. The reason for this non-linearity is the S/D resistance, adding as a series resistance to channel resistance and becoming more profound for narrow channels and short gate lengths [20]. This effect is important and has to be taken into account while analyzing the mobility graphs.
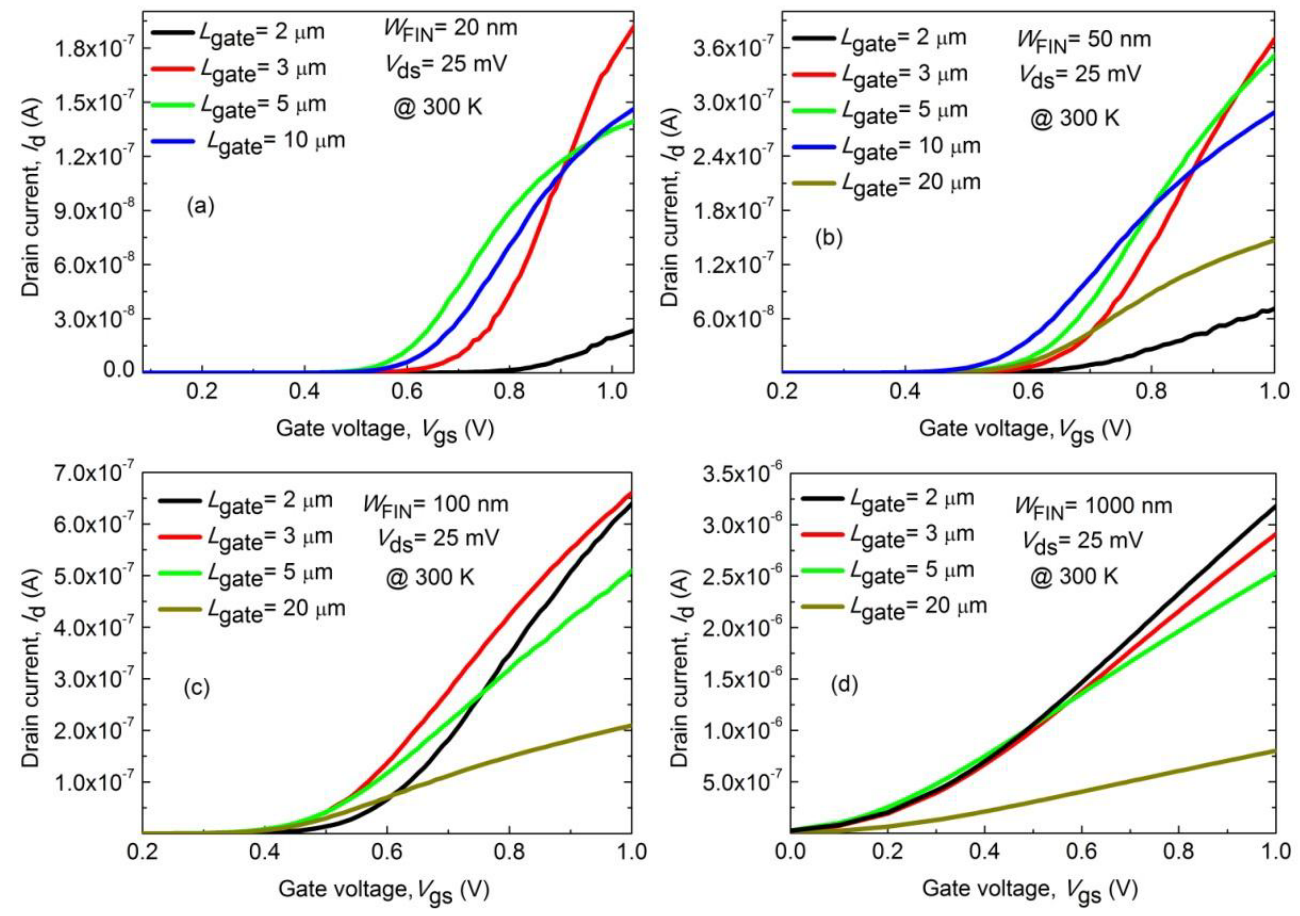

Fig. $4.16 I_{d}-V_{g s}$ device characteristics for $L_{\text {gate }}=2-20 \mu \mathrm{m}$ at $V_{d s}=25 \mathrm{mV}$ for 5-fin devices with a) $W_{\mathrm{FIN}}=20 \mathrm{~nm}$, b) $W_{\mathrm{FIN}}=50 \mathrm{~nm}$, c) $W_{\mathrm{FIN}}=100 \mathrm{~nm}$, and d) $W_{\mathrm{FIN}}=1000 \mathrm{~nm}$. The measurements were performed at $300 \mathrm{~K}$.

The effective mobility is plotted against the surface electric field of Si. Since an ensemble of electrons that is averaged over the inversion layer, the measured mobility is often called "effective" mobility. Since our FinFETs are fully depleted the surface electric field of Si perpendicular to the current flow direction is given by:

$$
E=\frac{Q_{i n v}}{\varepsilon_{S i}} \approx \frac{C_{o x}\left(V_{g s}-V_{t h}\right)}{\varepsilon_{S i}},
$$


where $Q_{\text {inv }}$ is the inversion charge per unit area. Note that Eq. (4.16) does not represent the effective field [21], rather the maximum field in the Si. By using the Eq. (4.14) and Eq. (4.16), $\mu_{e f f}$ and $E$ can be calculated. The $\mu_{e f f}-E$ curves for different fin widths and gate lengths are plotted in Fig. 4.17.
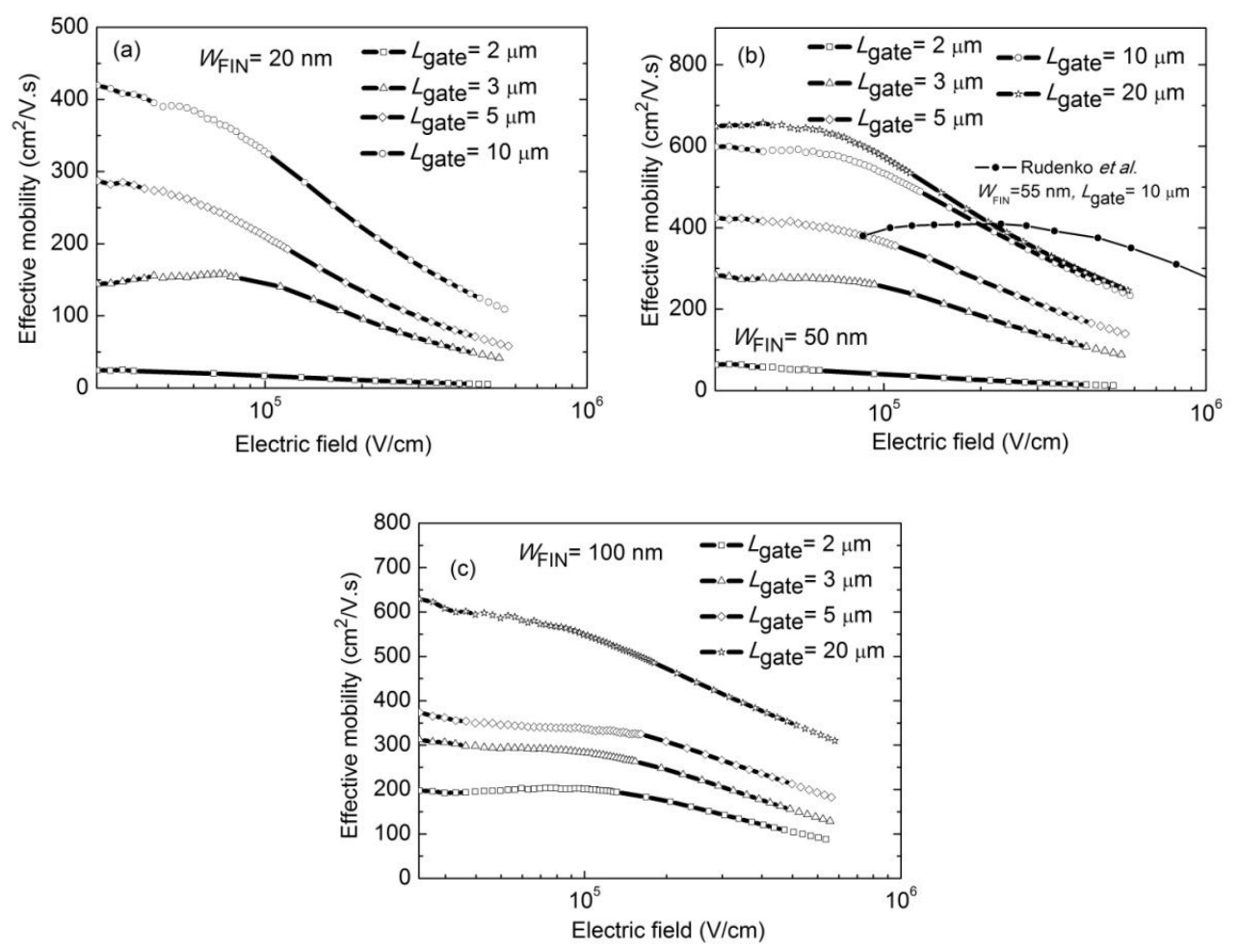

Fig. 4.17 Effective mobility-electric field curves of 5-fin devices with (a) $W_{\text {FIN }}=20 \mathrm{~nm}$, (b) $W_{\text {FIN }}=50$ $\mathrm{nm}$ and (110) n-FinFET with $W_{\mathrm{FIN}}=55 \mathrm{~nm}$ obtained from Rudenko et al. [22], (c) $W_{\mathrm{FIN}}=100 \mathrm{~nm}$ and $L_{\text {gate }}=2-10 \mu \mathrm{m}$.

Figs. 4.17 (a)-(c) show that effective mobility is depending on the $W_{\text {FIN }}$ and $L_{\text {gate }}$. Short channel effects make the calculations less accurate, especially for wide fins. Therefore, the effective mobility-electric field curve is not shown for a $1000 \mathrm{~nm}$ wide device.

In Fig. 4.17 (b), when $W_{\mathrm{FIN}}=50 \mathrm{~nm}$, the highest effective mobility is obtained. An increase in effective mobility for a narrow fin device was expected since the carriers are being transported through the body (i.e. volume inversion), encountering less surface scattering. However, if the fin width decreases below $10 \mathrm{~nm}$, the body size becomes so small that the electron transport also occurs at the surfaces, resulting in a lower mobility $[23,24]$. Although the volume is still large enough for the specified $W_{\mathrm{FIN}}=20 \mathrm{~nm}$ to neglect this effect, it is shown in Fig. 4.17 (a) that the mobility for this device is lower than the device with $W_{\mathrm{FIN}}=50 \mathrm{~nm}$. This can be attributed by the smaller and irregularly distributed 
actual fin width over the fin height as shown in our TEM analysis (Fig. 4.10 (c)) and possibly due to the series resistance effect, as mentioned before in section 4.2.3. For comparison, effective mobility-electric field curve presented by Rudenko et al. [22] is also drawn in Fig. 4.17 (b). Although the effective mobility values are comparable to the calculated values in this work, the field dependence of the mobility is different. The discrepancy at high fields could be due to an increased surface scattering caused by the irregular fin pattern for our devices.

Especially for $20 \mathrm{~nm}$ and $50 \mathrm{~nm}$ wide devices, the mobility for the $2 \mu \mathrm{m}$ gate length is very low, since the effect of the S/D resistance is more profound for narrow channel devices with short gate lengths (see Fig. 4.17 (a)). The mobility shows a dependence on the gate length also. As can be seen in Figs. 4.17 (a)-(c), devices with a longer gate length have a higher mobility for all fin widths. As mentioned before, the S/D extension resistances add as a series resistance to the channel resistance at high fields (in this case above $\approx 6 \times 10^{4}$ $\mathrm{V} / \mathrm{cm}$ ). However, below this field the effect of the S/D resistance is not important. Another possible reason for mobility degradation could be negative fixed or mobile charges at the gate edges close to the S/D regions [25]. This is highly possible for our devices since an increase in the threshold voltage is observed for shorter gate lengths.

\subsubsection{C-V characterization}

Dedicated $C-V$ test structures with GSG connections together with their open and short de-embedding pairs were designed for different dimensions. Each capacitance measurement was performed by de-embedding the pad as well as the parasitic connections. The capacitor dimensions are summarized in Table 4.3, section 4.1.1. The measurements were performed at $V_{a c}=20 \mathrm{mV}$ and at $1 \mathrm{MHz}$ frequency.

Fig. 4.18 shows the $C-V$ curves obtained from planar capacitors for $W_{\text {FIN }}=4 \mu \mathrm{m}, 10 \mu \mathrm{m}, 20$ $\mu \mathrm{m}$ and $L_{\text {gate }}=20 \mu \mathrm{m}$. The specific oxide capacitance was calculated using Eq. (4.15) by changing the value of the capacitor area. The calculated and measured capacitance values at strong inversion are presented in Table 4.4. 


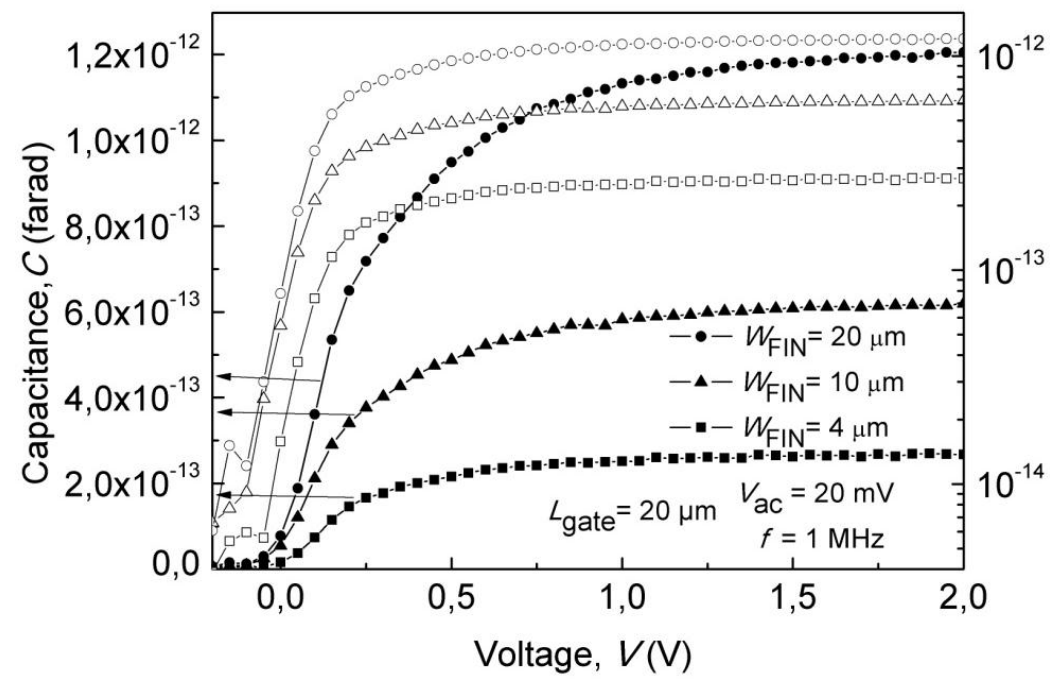

Fig. $4.18 C-V$ characteristics of capacitance test structures for $W_{\mathrm{FIN}}=4 \mu \mathrm{m}, 10 \mu \mathrm{m}, 20 \mu \mathrm{m}$ and $L_{\text {gate }}=$ $20 \mu \mathrm{m}$. Graphs are plotted in linear and logarithmic scale. Measurements were performed at $f=1$ $\mathrm{MHz}$ with $V_{a c}=20 \mathrm{mV}$.

Table 4.4 Calculated and measured (strong inversion) capacitance values for planar C-V test structures

\begin{tabular}{ccc}
\hline \hline & $\begin{array}{c}\text { Calculated } \\
\text { Capacitance }\end{array}$ & $\begin{array}{c}\text { Measured } \\
\text { Capacitance }\end{array}$ \\
\hline \hline$W_{\text {FIN }}=4 \mu \mathrm{m}, L_{\text {gate }}=20 \mu \mathrm{m}$ & $2.5 \times 10^{-13} \mathrm{~F}$ & $2.7 \times 10^{-13} \mathrm{~F}$ \\
$W_{\text {FIN }}=10 \mu \mathrm{m}, L_{\text {gate }}=20 \mu \mathrm{m}$ & $5.9 \times 10^{-13} \mathrm{~F}$ & $6.1 \times 10^{-13} \mathrm{~F}$ \\
$W_{\text {FIN }}=20 \mu \mathrm{m}, L_{\text {gate }}=\mathbf{2 0} \boldsymbol{\mu m}$ & $1.2 \times 10^{-12} \mathrm{~F}$ & $1.2 \times 10^{-12} \mathrm{~F}$ \\
\hline \hline
\end{tabular}

The calculated and measured capacitance values for each capacitor are almost equal. Hence, the measured capacitance at strong inversion mainly includes the oxide capacitance. The amount of interface traps associated to the sub-threshold slope was calculated in section 4.2.4.

The same measurements were performed using parallel fins with narrow fin widths. The dimensions of these test structures are given in Table 4.5. The measurements on parallel fin capacitors were also performed at $f=1 \mathrm{MHz}$, with $V_{a c}=20 \mathrm{mV}$ and the results are shown in Fig. 4.19. In this figure, the capacitance values for 20,40 and 60 parallel fins with $W_{\text {FIN }}=$ $20 \mathrm{~nm}, 30 \mathrm{~nm}, 40 \mu \mathrm{m}$ and $L_{\text {gate }}=20 \mu \mathrm{m}$ are plotted. The calculated and measured capacitance values at strong inversion are given in Table 4.5. 

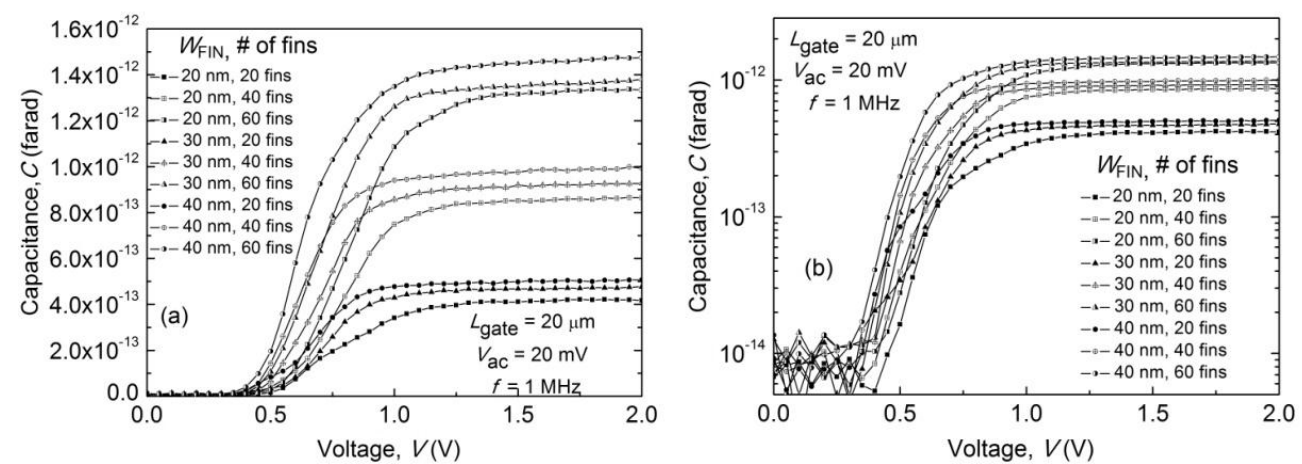

Fig. 4.19 C-V characteristics of capacitor test structures for different areas, scaling with $W_{\text {FIN }}$ and number of fins in (a) linear and (b) logarithmic scale. Measurements were performed at $f=1 \mathrm{MHz}$ with $V_{a c}=20 \mathrm{mV}$.

Table 4.5 (a) Calculated and (b) measured capacitance values for parallel fin C-V test structures

\begin{tabular}{|c|c|c|c|}
\hline (a) Calculated & $\begin{array}{c}\text { Capacitance } \\
\text { for } 20 \text { fins }\end{array}$ & $\begin{array}{l}\text { Capacitance } \\
\text { for } 40 \text { fins }\end{array}$ & $\begin{array}{c}\text { Capacitance } \\
\text { for } 60 \text { fins }\end{array}$ \\
\hline$W_{F I N}=20 \mathrm{~nm}, L_{\text {gate }}=20 \mu \mathrm{m}$ & $4.0 \times 10^{-13} \mathrm{~F}$ & $8.0 \times 10^{-13} \mathrm{~F}$ & $1.2 \times 10^{-12} \mathrm{~F}$ \\
\hline$W_{F I N}=40 \mathrm{~nm}, L_{g a t e}=20 \mu \mathrm{m}$ & $4.3 \times 10^{-13} \mathrm{~F}$ & $8.5 \times 10^{-13} \mathrm{~F}$ & $1.3 \times 10^{-12} \mathrm{~F}$ \\
\hline$W_{F I N}=60 \mathrm{~nm}, L_{\text {gate }}=20 \mu \mathrm{m}$ & $4.5 \times 10^{-13} \mathrm{~F}$ & $9.0 \times 10^{-13} \mathrm{~F}$ & $1.4 \times 10^{-12} \mathrm{~F}$ \\
\hline (b) Measured & $\begin{array}{c}\text { Capacitance } \\
\text { for } 20 \text { fins }\end{array}$ & $\begin{array}{c}\text { Capacitance } \\
\text { for } 40 \text { fins }\end{array}$ & $\begin{array}{c}\text { Capacitance } \\
\text { for } 60 \text { fins }\end{array}$ \\
\hline$W_{F I N}=20 \mathrm{~nm}, L_{\text {gate }}=20 \mu \mathrm{m}$ & $4.1 \times 10^{-13} \mathrm{~F}$ & $8.6 \times 10^{-13} \mathrm{~F}$ & $1.3 \times 10^{-12} \mathrm{~F}$ \\
\hline$W_{F I N}=40 \mathrm{~nm}, L_{\text {gate }}=20 \mu \mathrm{m}$ & $4.7 \times 10^{-13} \mathrm{~F}$ & $9.3 \times 10^{-13} \mathrm{~F}$ & $1.4 \times 10^{-12} \mathrm{~F}$ \\
\hline$W_{F I N}=60 \mathrm{~nm}, L_{\text {gate }}=20 \mu \mathrm{m}$ & $5.0 \times 10^{-13} \mathrm{~F}$ & $9.9 \times 10^{-13} \mathrm{~F}$ & $1.5 \times 10^{-12} \mathrm{~F}$ \\
\hline
\end{tabular}

The calculated capacitance values are in agreement with the measured (strong inversion) values shown in Fig. 4.19. No significant difference is observed in the slope, which points out that the trap density has not changed much. The difference observed between Fig. 4.18 and Fig. 4.19 is the threshold voltage shift of about $0.4 \mathrm{~V}$. The shift $C-V$ curves can be attributed to irregular fin pattern (see Fig. 4.10 (c)) and higher amount of surface charge. Both these non-idealities are related to the processing. Each fin has two sidewalls which were formed in the dry etching process. This type of etching process results in surface imperfections. Compared to planar capacitors, parallel narrow fin capacitors are more 
eligible for surface imperfections and interface charges, which explain the shift in Fig. 4.19. In addition, as explained in section 4.2.3, narrow fin devices have a limited amount of inversion charge, which results in a decrease of the sub-threshold current and hence, a positive shift.

\subsection{Conclusions}

In this chapter, the fabrication and characterization of FinFETs are presented. As a first step in the processing, the (001) surface orientated SOl layer was thinned down to 150 $\mathrm{nm}$. After patterning the $\mathrm{Si}$ fins with EBL, a gate oxide layer was thermally grown and a $\mathrm{TiN}+\alpha$-Si deposition was done. After patterning the gate metal, ion implantation and RTA were performed. As a final step, contact pads were defined by Al-Si (1\%) sputtering through a shadow mask.

Physical and electrical characterization of the fabricated structures has been discussed showing that devices have a Si body of down to $14 \mathrm{~nm}$ width and an aspect ratio of up to 7.5. The TEM analysis also shows that the FinFETs comprise a well defined surrounding gate stack. Resistivity measurements on dedicated process control test structures show that the sheet resistances of the S/D regions as well as gate stack are well in line with TCAD simulations.

The fabricated devices were further analyzed by $I-V$ and $C-V$ measurements. Electrical measurements revealed that 3 out of 5 devices function well. SS of $I_{d}-V_{g S}$ curves for the devices with $W_{\mathrm{FIN}}=20-100 \mathrm{~nm}$ and $L_{\text {gate }}=3 \mu \mathrm{m}$ is determined to be $76 \mathrm{mV} /$ dec. A low leakage and high $I_{\text {on }} / I_{\text {off }}\left(\approx 10^{8}\right)$ together with a DIBL of $93 \mathrm{mV} / \mathrm{V}$ are obtained. These values as well as the comparison with the NXP-FinFETs indicate that the fabricated FinFETs have a satisfactory performance.

The gate length scaling of the drain current is discussed and shown for above-threshold region. The results indicate that, for devices with narrow fins and short gate lengths, the $S / D$ resistance becomes more profound. In addition, higher threshold voltages are obtained which can be attributed to the irregular fin width pattern directly or indirectly leading to fixed charge or traps at the gate edges near the S/D regions. These charges are more effective for short channel gates. As a result, a decrease in drain current is observed. However, for devices with wide fins, the current is affected by short channel effects because of the combination of a lowly doped silicon body and poor electrostatic gate control. The effective mobility-electric field curve for long gate lengths $(\approx 10 \mu \mathrm{m})$ show comparable values as reported in the literature.

$C-V$ analysis has been performed on dedicated test capacitors. Good agreement has been obtained with calculated and measured oxide capacitance values, for both planar and 
narrow fin capacitors. A threshold voltage shift is observed for narrow fin structures. This effect is due to narrow channel's limited amount of carriers, which is more profound due to irregular fin width over the fin height, and higher negative charges at the fin sidewalls due to the dry etching process.

\section{References}

[1] D. K. Schroder, "Semiconductor Material and Device Characterization", $3^{\text {rd }}$ ed., Wiley interscience, 2006.

[2] L. van der Pauw, "A method of measuring specific resistivity and Hall Effect of discs of arbitrary shape", Philips Research Reports, 13, pp. 1-9, 1958.

[3] W. Versnel, "Analysis of symmetric Van der Pauw structures with finite contacts", Solid State Electronics, 21, pp. 1261-1268, 1978.

[4] S. Wolf and R. N. Tauber, "Silicon processing for the VLSI era", 2, Sunset beach: Lattice press, 1986.

[5] N. Stavitski, "Silicide-to-silicon specific contact resistance characterization", PhD thesis, University of Twente, December 2009.

[6] T. A. Schreyer, and K. C. Saraswat, "A two dimentional Analytical Model of the Cross bridge Kelvin Resistor", IEEE Electron Device Letters, 7, pp. 661-663, 1986.

[7] M. Finetti, A. Scorzoni and G. Soncini, "Lateral Current Crowding Effects on Contact Resistance Measurements in 4 terminal Resistor Test Patterns", IEEE Electron Device Letters, 5, pp. 524-526, 1984.

[8] S. M. George, "Atomic Layer Deposition: An Overview”, Chem. Rev., 110, pp. 111-131, 2010.

[9] C. Y. Kang, R. Choi, M. M. Hussain, J. Wang, Y. J. Suh, H. C. Floresca, M. J. Kim, J. Kim, Jiyoung, B. H. Lee, and R. Jammy, "Effects of metal gate-induced strain on the performance of metal-oxidesemiconductor field effect transistors with titanium nitride gate electrode and hafnium oxide dielectric", Applied Physics Letters, 91, pp. 033511-033511-3, 2007.

[10] S. M. Sze, and K.K. Ng, "Physics of semiconductor devices", $3^{\text {rd }}$ ed., 2007.

[11] H. van Bui, A. Y. Kovalgin, and R. A. M. Wolters, "On the difference between optically and electrically determined resistivity of ultrathin TiN films", Applied Surface Science, 269, pp. 45-49, 2013.

[12] M. Miyawaki, S. Yoshitake, and T. Ohmi, "Improvement of Aluminum-Si Contact Performance in Native-Oxide-Free Processing”, IEEE Electron Device Letters, 11, pp.448-450, 1990.

[13] Y. Taur, X. Liang, W. Wang, and H. Lu, "A Continuous, Analytic Drain-Current Model for DG MOSFETs", IEEE Electron Device Letters, 25, pp. 107-109, 2004.

[14] C. Gustin, A. Mercha, J. Loo, V. Subramanian, B. Parvais, M. Dehan ,and S. Decoutere, "Stochastic Matching Properties of FinFETs", IEEE Electron Device Letters, 27, pp. 846-848, 2006.

[15] E. Baravelli, A. Dixit, R. Rooyackers, M. Jurczak, N. Speciale, and K. De Meyer, "Implications of Fin Width Scaling on Variability and Reliability of High-k Metal Gate FinFETs" IEEE Transactions on Electron Devices, 54, pp. 2466-2474, 2007.

[16] S. Chabukswar, D. Maji, C. R. Manoj, K. G. Anil, V. Ramgopal Rao, F. Crupi, P. Magnone, G. Giusi, C. Pace, and N. Collaert, "Implications of fin width scaling on variability and reliability of high-k metal gate FinFETs", Microelectronic Engineering, 87, pp. 1963-1967, 2010.

[17] W. Xiong, "FinFETs and other multi gate transistors, Chapter 2: Multigate MOSFET Technology", Edited by J.-P. Colinge, Springer, 2008.

[18] E. J. Nowak, I. Aller, T. Ludwig, K. Kim, R. V. Joshi, Te C. Ching, K. Bernstein, and R. Puri, "Turning Silicon on its edge Double Gate CMOS/FinFET technology", IEEE Circuits and Devices Magazine, 20, pp. 20-31, 2004. 
[19] C. W. Lee, S. R. N. Yun, C. G. Yu, J. T. Park, and J. P. Colinge, "Device Design Guidelines for NanoScale MuGFETs", Solid-State Electronics, 51, pp. 505-510, 2007.

[20] A. A. Frantsuzov, N. I. Boyarkina, and V. P. Popov, "Decrease in effective electron mobility in the channel of a metal-oxide-semiconductor transistor as the gate length is decreased", Semiconductors, 42, pp. 215-219, 2008.

[21] A. G. Sabnis, and J. T. Clemens, "Characterization of the Electron Mobility in the Inverted (100) Si Surface", Technical Digest International Electron Devices Meeting (IEDM), pp. 18-21, 1979.

[22] T. Rudenko, V. Kilchytska, N. Collaert, M. Jurczak, A. Nazarov, and D. Flandre, "Carrier Mobility in Undoped Triple-Gate FinFET Structures and Limitations of Its Description in Terms of Top and Sidewall Channel Mobilities", IEEE Transactions on Electron Devices, 55, pp. 3532-3541, 2008.

[23] F. Gamiz, J. B. Roldan, J. A. Lopez-Villanueva, P. Cattujo-Cassinello, J. E. Carceller, P. Cartujo, and F. jimenez-Molinos, "Electron Transport in Silicon-on-Insulator Devices", Solid State Electronics, 45, pp. 613-620, 2001.

[24] L. Ge, J. G. Fossum, and F. Gamiz, "Mobility Enhancement via Volume Inversion in Double-gate MOSFETs", Proceedings of the IEEE International SOI conference, pp. 153-154, 2003.

[25] A. Nazarov, J.-P. Colinge, F. Balestra, and J. P. Raskin, "Semiconductor-On-Insulator Materials for Nanoelectronics Applications", Springer, p.314, 2011. 


\section{The PiezoFET: a piezoelectric layer around a FinFET}

In this chapter, the integration and use of a piezoelectric layer as a channel stressor is discussed. A novel four terminal (4T) device structure, called the PiezoFET, will be introduced. The process integration steps and device characteristics of this device are presented. No harmful effects have been observed in device properties after deposition of the piezoelectric material PZT. A high piezoelectric response in the range of $100 \mathrm{pm} / V$ has been obtained for the PZT PiezoFET evidencing the converse piezoelectric effect in the $4 T$ device. As expected, for the AIN PiezofET the response was less $(13 \mathrm{pm} / \mathrm{V})$. Furthermore, a 20-50\% change in the electron mobility and a drastic change in the subthreshold swing (up to 20\%) has been observed. The major effect on both mobility and subthreshold swing can be attributed to the strain formed by the difference in thermal expansion coefficient of Si and the PZT layer. It is also partly affected by the bias over the piezoelectric layer, which indicates converse piezoelectric effect related strain in both the silicon channel and gate oxide. 


\subsection{The piezoelectric layer as a stressor}

As discussed in chapter 3, ferroelectric materials are of high interest for device applications. In this chapter, we are interested in the converse piezoelectric effect of a commonly used ferroelectric material, called lead-zirconate-titanate $\left(\mathrm{Pb}\left[\mathrm{Zr}_{\mathrm{x}} \mathrm{Ti}_{1-\mathrm{x}}\right] \mathrm{O}_{3}\right)$ or in short PZT. PZT was chosen because of its high piezoelectric response among other piezoelectric materials [1]. It has been recently reported that the piezoelectric properties can be utilized for a strained silicon (Si) device application [2].

The converse piezoelectric coefficient of a material relates the mechanical displacement to the applied electric field [3]. The deformation can be along or perpendicular to the applied field, i.e. the longitudinal or transverse piezoelectric effect. The strain at the channel of a FET changes the electrical device characteristics. Using the reports of Skotnicki et al. [4] and Serra et al. [5], the effect of stress configurations in FinFETs on the band alignment and mobility in Si can be discussed. Although a planar structure has been used in [4], the results can be applied to a 3D structure like a FinFET keeping in mind the importance of the aspect ratio in mind.

Fig. 5.1 shows a schematic cross section of the PiezoFET with two Si fins under applied bias across a homogeneously deposited PZT layer. The PiezoFET is basically a four terminal device consisting of two separate gates: the conventional gate which we call now the bottom gate (BG), and a top gate (TG, or "pigate").

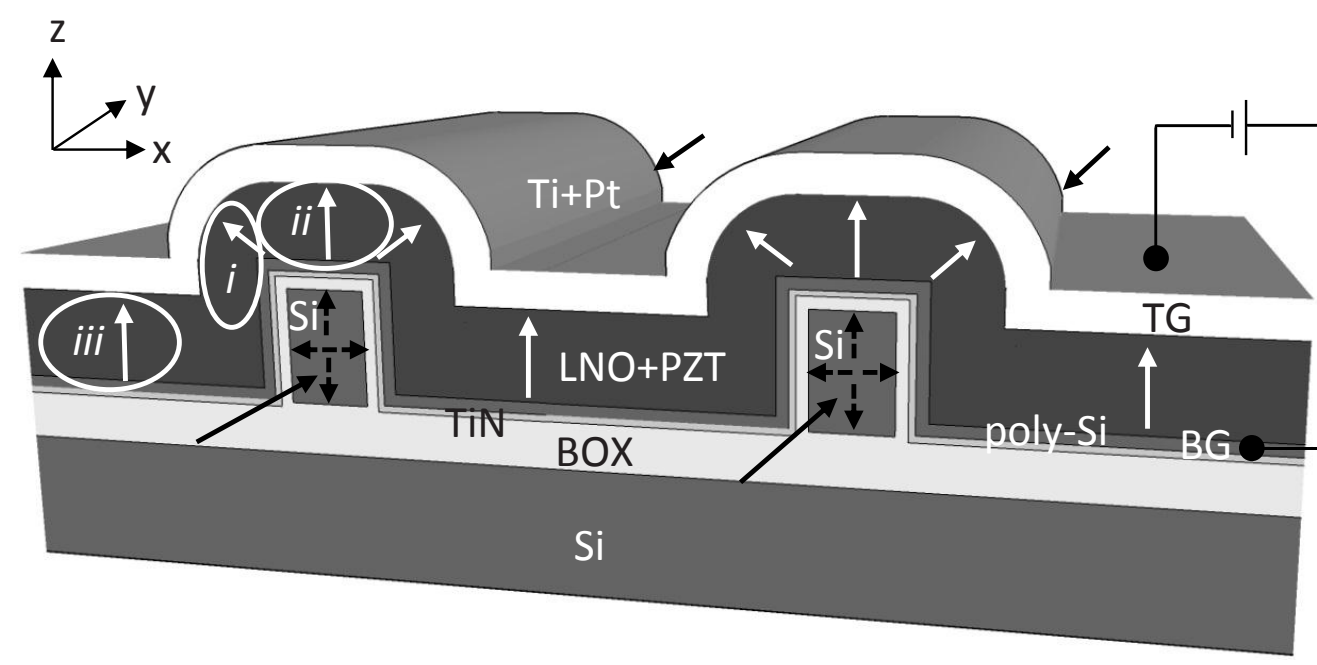

Fig. 5.1 PiezoFET schematic cross-section with narrow fins illustrating the formed strain condition corresponding to an upward piezoelectric response. The white arrows show the PZT deformation while the black arrows show the corresponding formed stress in the silicon fins. The figures are not to scale. 
Fig. 5.1 shows the displacements when the PZT is poled in the direction of the electric field between BG and TG. The white arrows indicate the piezoelectric displacement and strain in the PZT layer, while the black arrows indicate the corresponding stress formed inside the Si fins. It is plotted such that the PZT on the BOX layer (and at the fin sidewalls) determines the net component.

This schematic is drawn when there is a negative bias over the PZT layer $\left(V_{\pi}=V_{\mathrm{TG}}-V_{\mathrm{BG}}<0\right.$ V). In this case, the stress formed in the Si fin can be described as follows: $i$ ) due to the PZT layer on the fin sidewall region, the Si fin is under biaxial tensile stress along $x$ (fin width) and $z$ (fin height) and under uniaxial compressive stress along $y$ (channel length); ii) due to the PZT layer on top of the fin, biaxial compressive stress along $x$ and $y$ is formed and $\mathrm{iii)}$ due to the layer on the BOX region, tensile stress in Si along $x$ and compressive stress along $y$ is formed.

The overall effect depends on the fin width and aspect ratio. For narrow fin structures condition i) dominates whereas for a planar structure, condition ii) is more important due to a larger surface area. In practice, condition iii) would not be important for narrow fins, as will be explained later. For each fin width, the net stress depends on the amount of the individual stress components. For downward deflection (at positive bias over the PZT layer $\left(V_{\pi}=V_{\mathrm{TG}}-V_{\mathrm{BG}}>0 \mathrm{~V}\right)$ ), all the stress components change direction.

While drawing this strain illustration, the stress relieving effect of the intermediate layers was not taken into account. Lower stress is expected when thick electrodes and intermediate layers are used for the same device configuration. In addition, stress does not form strain if the Si fin is clamped to the substrate, e.g. along the $y$-axis. On the other hand, the BOX layer is relatively soft and allows the Si fin to be stressed.

To increase the mobility, an optimum configuration needs to be found regarding the orientation of the channel with respect to stress (see chapter 1, Fig. 1.2). The FinFET geometry is important as well. In narrow fin devices the sidewalls dominate in determining the stress whereas for wide fins stress formed from the top of the fins is more profound. In general, wider fins have a lower amount of stress from piezoelectric actuation since the main stress component is formed by the PZT on the BOX that is a soft material [2].

To obtain high strain levels in $\mathrm{Si}$, the stressor material needs to be as close as possible to the channel. This prevents stress relaxation due to the inter-layers. Stress also gradually decreases from top to bottom electrode in the PZT layer due to the clamping effect at the lower boundary. Therefore, the thickness and the choice of material between Si channel and PZT is very important. These have been optimized in the present state-of-art technology as described in chapter 3. The processing for the PiezoFETs has been carried out accordingly. 


\subsection{Processing of the PiezoFET}

\subsubsection{Mask design}

Fig. 5.2 shows the schematic mask layout of a typical PiezoFET including its design parameters and table 5.1 gives an overview of the designed mask dimensions used in our studies.

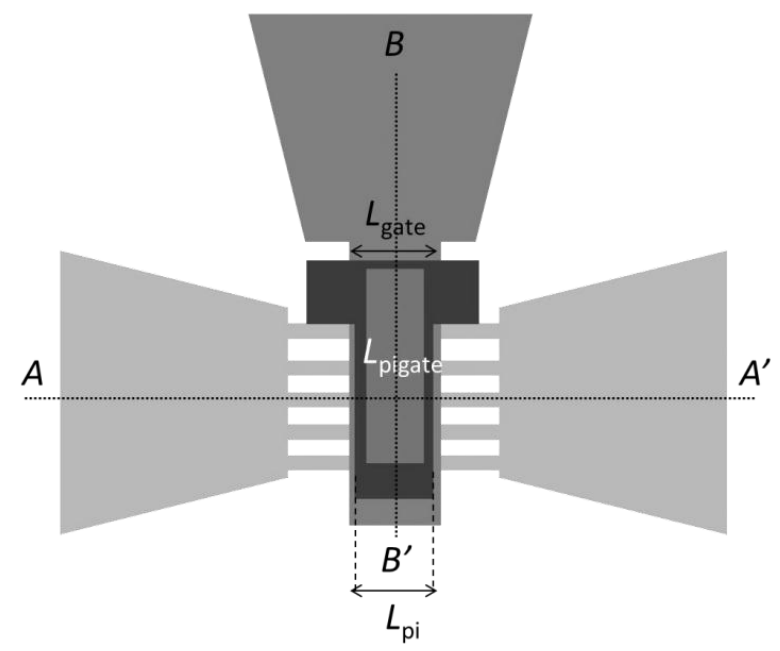

Fig. 5.2 Top view schematic layout of the PiezoFET with its design parameters. The figure is not to scale.

Table 5.1 Summary of the designed PiezoFET mask dimensions. $L_{\text {gate, }} L_{\mathrm{pi}}$ and $L_{\text {pigate }}$ represent the bottom gate length, piezoelectric layer length and top gate length, respectively.

\begin{tabular}{lccl}
\hline & $\boldsymbol{L}_{\text {gate }}$ & $\boldsymbol{L}_{\boldsymbol{p} \boldsymbol{i}}$ & $\boldsymbol{L}_{\text {pigate }}$ \\
\hline \hline PiezoFET-1 & $10 \mu \mathrm{m}$ & $8 \mu \mathrm{m}$ & $3 \mu \mathrm{m}$ \\
PiezoFET-2 & $15 \mu \mathrm{m}$ & $13 \mu \mathrm{m}$ & $4 \mu \mathrm{m}$ \\
PiezoFET-3 & $20 \mu \mathrm{m}$ & $18 \mu \mathrm{m}$ & $5 \mu \mathrm{m}$ \\
PiezoFET-4 & $30 \mu \mathrm{m}$ & $28 \mu \mathrm{m}$ & $10 \mu \mathrm{m}$ \\
\hline \hline
\end{tabular}

Two different mask sets were designed for the fabrication. In the first design the piezoelectric layer dimensions were larger than the bottom gate. Using this mask set, the first PZT/LNO stack deposition and characterization experiments were carried out on FinFETs. The first observation was a serious underetch of the PZT layer, which would limit the top contact length. Moreover, if the PZT was patterned larger than the TiN (bottom) gate, the conductive LNO layer forms a short between the source (S) and drain (D) regions. Although the gate oxide layer on the S/D extensions of FinFETs should prevent this short, the dielectric properties deteriorated during the implantation or gate etching 
processes. Therefore, a $2^{\text {nd }}$ mask set was designed regarding these effects and presented in this section.

In the modified design (Table 5.1), the lateral dimensions of the piezoelectric layer $\left(L_{\mathrm{pi}}\right)$ have been kept larger than the top gate $\left(L_{\text {pigate }}\right)$ because of the 2-3 $\mu \mathrm{m}$ lateral under etch of the PZT layer. However, although devices with four different gate lengths were designed, the ones with $L_{\text {pigate }}=3 \mu \mathrm{m}$ and $4 \mu \mathrm{m}$ were found to be leaky (PiezoFET-1 and 2). The small overlap between the top gate and piezoelectric layer could be the reason for the relatively high leakage between top and bottom gates. PiezoFET-3 and 4 were used in this work.

To compare the piezoelectric effect on FinFETs and on planar structures, test structures with different geometries and areas have also been designed. These structures are equivalent to the ones studied in chapter 3. Likewise, capacitors with different areas were designed including open and short de-embedding structures. The designed test structures consist of a so-called Ground-Signal-Ground (GSG) configuration, commonly used for high frequency measurements. Fig. 5.3 illustrates the designed test structures for planar $C-V$ measurements as well as its de-embedding pairs. The top GSG pads are connected to the bottom (TiN) gate of the FinFET, while the bottom GSG pads are connected to the top gate. Table 5.2 summarizes the dimensions of the designed test capacitors.
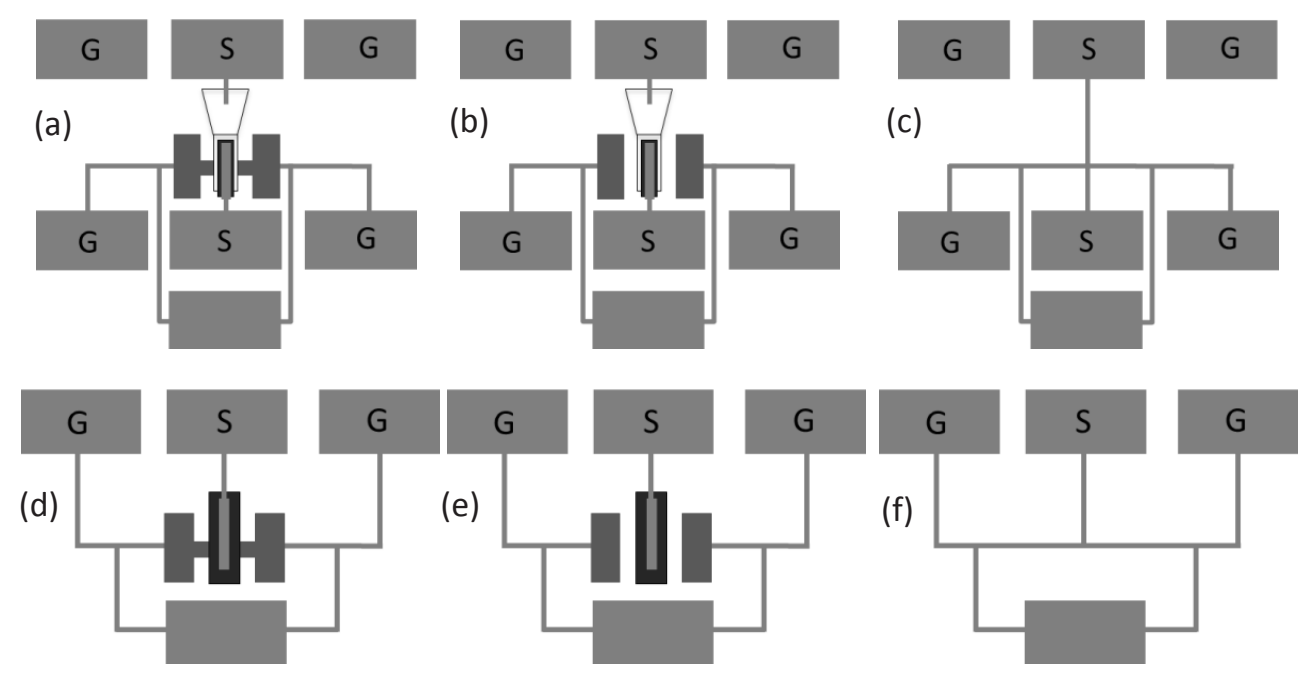

Fig. 5.3 Ground-signal-ground (GSG) $C-V$ test structures for the $C-V$ measurement of (a) the piezoelectric layer in an actual PiezoFET and its (b) open de-embedding, (c) short de-embedding structures; and those for $C-V$ measurement of (d) the planar piezoelectric layer and its (b) open deembedding, (f) short de-embedding structures. 
Table 5.2 Design parameters of the C-V test structures

\begin{tabular}{|c|c|c|c|}
\hline $\begin{array}{l}\text { number } \\
\text { of fins }\end{array}$ & $W_{F I N} \& L_{\text {gate }} \& L_{\text {pigate }}$ & $W_{\text {FIN }} \& L_{\text {gate }} \& L_{\text {pigate }}$ & $\overline{W_{\text {FIN }} \& L_{\text {gate }} \& L_{\text {pigate }}}$ \\
\hline 1 & $20 \mu \mathrm{m} \& 20 \mu \mathrm{m} \& 4 \mu \mathrm{m}$ & $10 \mu \mathrm{m} \& 20 \mu \mathrm{m} \& 4 \mu \mathrm{m}$ & $4 \mu \mathrm{m} \& 20 \mu \mathrm{m} \& 4 \mu \mathrm{m}$ \\
\hline 20 & $20 \mathrm{~nm} \& 20 \mu \mathrm{m} \& 4 \mu \mathrm{m}$ & $30 \mathrm{~nm} \& 20 \mu \mathrm{m} \& 4 \mu \mathrm{m}$ & $40 \mathrm{~nm} \& 20 \mu \mathrm{m} \& 4 \mu \mathrm{m}$ \\
\hline 40 & $20 \mathrm{~nm} \& 20 \mu \mathrm{m} \& 4 \mu \mathrm{m}$ & $30 \mathrm{~nm} \& 20 \mu \mathrm{m} \& 4 \mu \mathrm{m}$ & $40 \mathrm{~nm} \& 20 \mu \mathrm{m} \& 4 \mu \mathrm{m}$ \\
\hline 60 & $20 \mathrm{~nm} \& 20 \mu \mathrm{m} \& 4 \mu \mathrm{m}$ & $30 \mathrm{~nm} \& 20 \mu \mathrm{m} \& 4 \mu \mathrm{m}$ & $40 \mathrm{~nm} \& 20 \mu \mathrm{m} \& 4 \mu \mathrm{m}$ \\
\hline
\end{tabular}

\subsubsection{Processing}

To fabricate the PiezoFET, the piezoelectric PZT layer was deposited on top of $(1 \overline{1} 0) /[110]$ oriented FinFETs, presented in chapter 4 . The process scheme of the PiezoFET after the completed FinFET processing is depicted in Fig. 5.4.

(a) Al stripping

(b) LNO+PZT deposition \& etching

(c) Metallization

(a)

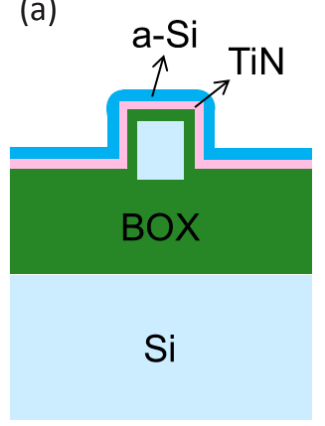

$B-B^{\prime}$ cross section (a)

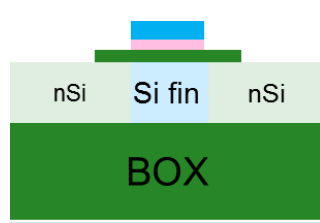

$\mathrm{Si}$

$A-A^{\prime}$ cross section

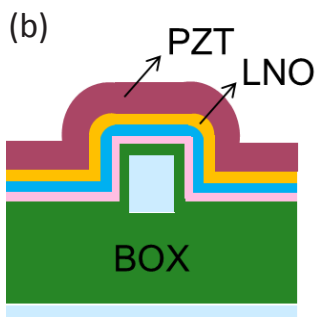

$\mathrm{Si}$

$B-B^{\prime}$ cross section (b)

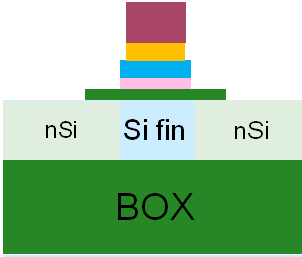

Si

$A-A^{\prime}$ cross section 


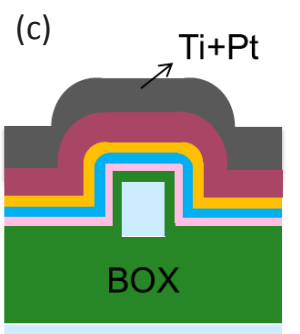

$\mathrm{Si}$

\section{$B-B^{\prime}$ cross section}

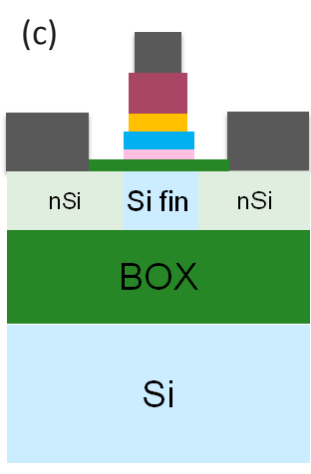

$A-A^{\prime}$ cross section

Fig. 5.4 Schematic process flow of the PiezoFET showing the process steps described in (a)-(c). The $A-A^{\prime}$ and $B-B^{\prime}$ cross sections are indicated in the top view schematic layout depicted in Fig. 5.2. The figures are not to scale.

The processing scheme can be summarized as follows:

(a) Al stripping: In this step, Al was stripped from the contacts pads since the PZT deposition temperature $\left(650^{\circ} \mathrm{C}\right)$ is not compatible with Al. The $250 \mathrm{~nm}$ Al was wet etched in standard phosphoric acid/acetic acid/nitric acid solution at $45{ }^{\circ} \mathrm{C}$ in $1 \mathrm{~min}$. As mentioned in chapter 4 , the metallization was done by using an Al-Si (1 \%) target for the FinFET processing. Therefore, after Al removal, Si precipitates are still present in the contact regions.

(b) LNO+PZT deposition \& etching: The $\mathrm{LaNiO}_{3}$ (LNO) layer and the PZT layer were deposited in the pulsed laser deposition (PLD) system developed by SolMates BV, Enschede, the Netherlands. The deposition temperature was kept constant at $650{ }^{\circ} \mathrm{C}$ during the consecutive deposition of these layers. A $10 \mathrm{~nm}$ thick LNO layer and a $100 \mathrm{~nm}$ thick PZT layer were deposited (see Fig. 5.6). After deposition, further process steps were performed as described in chapter 3: the PZT/LNO stack was patterned by wet etching using a 2 step process: (1) $5 \mathrm{sec}$ in $\mathrm{BHF}$ (12.5\%): $\mathrm{HNO}_{3}: \mathrm{NH}_{4} \mathrm{Cl}: \mathrm{H}_{2} \mathrm{O}$ (8:1:8:32) at room temperature and (2) $5 \mathrm{sec}$ in $\mathrm{HCl}(45 \%): \mathrm{H}_{2} \mathrm{O}(1: 1)$ at $45^{\circ} \mathrm{C}$. This 2 step process was repeated until the layer was completely etched. A $3 \mu \mathrm{m}$ under etch of the PZT layer was observed through the optical microscope.

(c) Metallization: After patterning the contacts with photolithography (PL), the oxide on the contact holes was removed by $1 \% \mathrm{HF}$ etching. A $10 \mathrm{~nm}$ thick Ti and $100 \mathrm{~nm}$ thick Pt metal layers were sputtered as a contact metal. Then, a lift off process was done in acetone. In the final structure, all the contact pads are formed by a Pt/Ti stack, as shown in the optical micrograph of a PiezoFET in Fig. 5.5. 


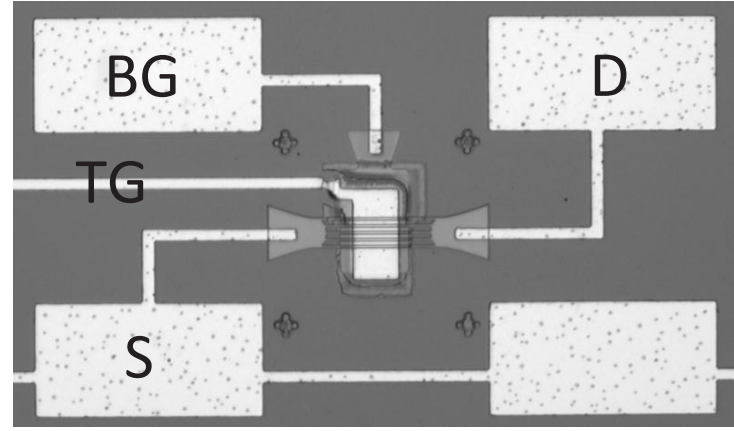

Fig. 5.5 Optical micrograph of a typical 5-fin PiezoFET. The bottom gate, top gate, drain and source are indicated by $B G, T G, D$, and $S$, respectively.

\subsection{Physical, optical and ferroelectric characterization of PZT on FinFETs}

\subsubsection{TEM analysis}

After sample preparation with the focused-ion beam system (FIB), cross section pictures were taken with a Transmission Electron Microscope (TEM). Fig. 5.6 shows TEM pictures of a 5-fin $20 \mathrm{~nm}$ and $100 \mathrm{~nm}$ wide PiezoFET with a $10 \mathrm{~nm}$ thick LNO and $100 \mathrm{~nm}$ thick PZT layer. All fins are imaged in Figs. 5.6 (a) and (d). Figs. 5.6 (b) and (e) show a magnified image of a single fin. The top gate is not visible in the figures due to the sample preparation (i.e. Pt protection layer). In this image, good step coverage of the TiN as well as poly-Si is recognized whereas the step coverage of the LNO/PZT stack is poor. This poor step coverage is due to the directional growth in the PLD system. This is obviously not an issue for the ALD deposition of TiN. Although the step coverage is poor, electrical measurements show that there is no direct electrical short circuit between the bottom gate (TiN) and the top gate Ti/Pt in the structures (see section 5.4.1).

To show each layer around the fin more clearly and to evaluate the crystal orientation of the PZT at the fin sidewalls, elemental as well as crystal analysis were done by the TEM. Figs. 5.6 (c) and (f) show the RGB (red, green, blue) map for the elemental analysis of a single fin. In this image, the La is shown in red; $\mathrm{Ti}$ is shown in green and $\mathrm{Si}$ is shown in blue. La is clearly observed on the top, while it gets thinner at the sidewalls of the fin. Figs. 5.6 (b) and (e) shows that the PZT layer thickness is decreased to 20-50 nm while the LNO layer thickness is decreased to $2-5 \mathrm{~nm}$ at the sidewalls. In Figs. 5.6 (g)-(h), a magnified image of the PZT layer at the sidewall and on top of the fin is shown. These images show that the PZT layer is poly-crystalline both at the fin sidewalls and on top of the fin. 

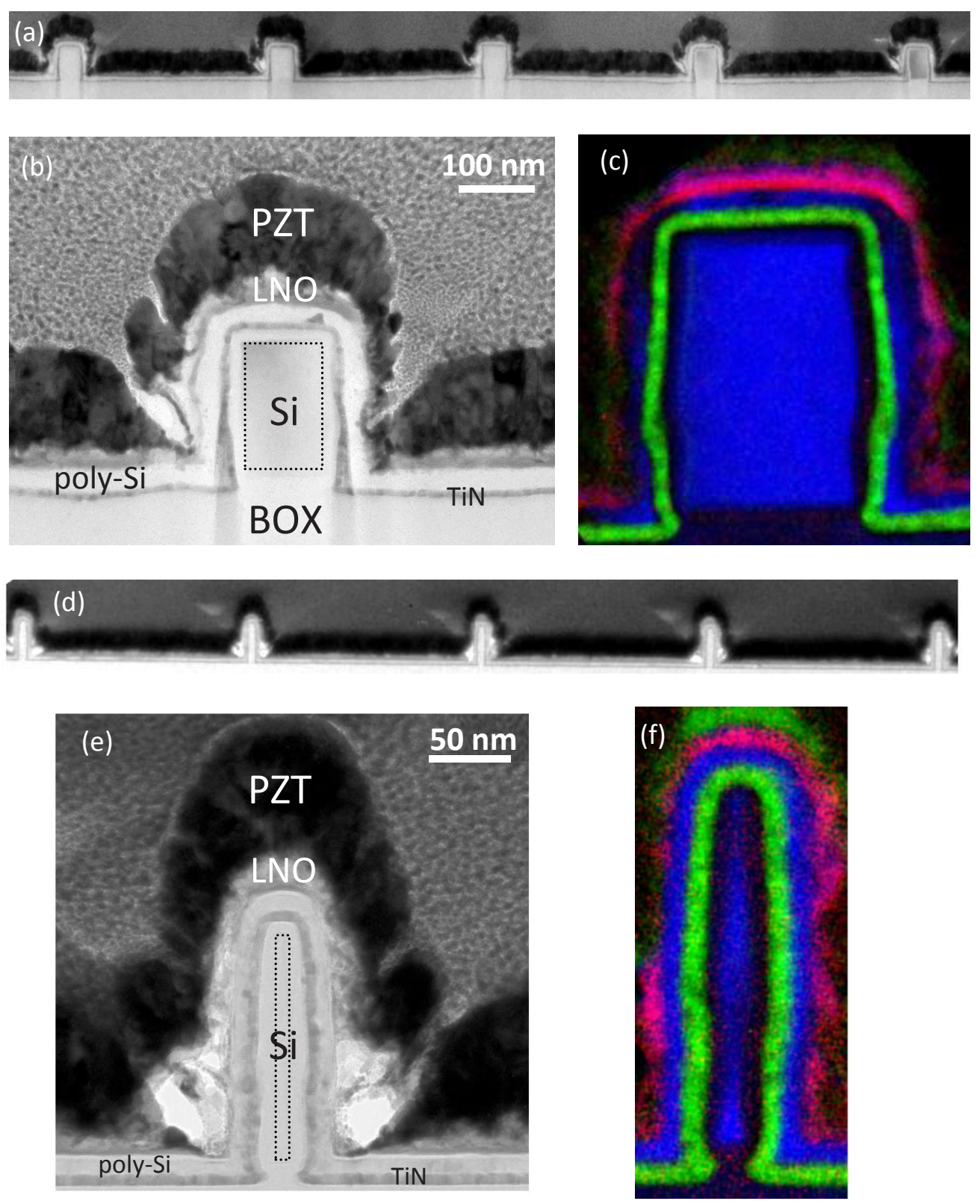

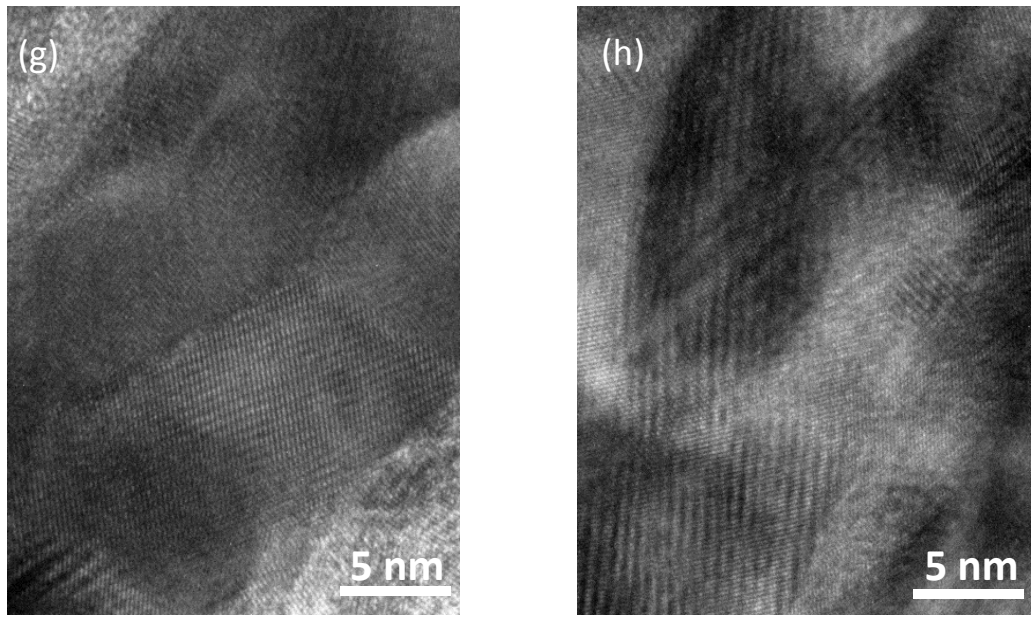

Fig. 5.6 HR-TEM images of a 5-fin PiezoFET with a $100 \mathrm{~nm}$ wide fin and a $10 \mathrm{~nm}$ LNO $+100 \mathrm{~nm}$ PZT layer stack: (a) zoomed out image of all fins, (b) magnified image of one fin (the dotted lines are drawn around the fin to guide the eye), (c) an RGB map of the fin (La, red; Ti, green; Si, blue), and with a $20 \mathrm{~nm}$ wide fin and a $10 \mathrm{~nm}$ LNO $+100 \mathrm{~nm}$ PZT layer stack: (d) zoomed out image of all fins, (e) magnified image of one fin (the dotted lines are drawn around the fin to guide the eye), (f) an RGB map of the fin (La, red; Ti, green; Si, blue), (g) magnified image of the PZT of the sidewall of the fin and (h) magnified image of the PZT on top of the fin. Figs. (g) and (h) are taken in the same direction as Figs. (b) and (e). They show that PZT is poly-crystalline both at the sidewall and top of the fin.

\subsubsection{Laser Doppler vibrometer measurements}

The laser Doppler vibrometer (LDV) measurement is important to characterize the piezoelectric response of the PZT layer, as was discussed in chapter 3 . In chapter 3 the PZT layer was characterized on a planar surface while in this chapter analyses are presented on $150 \mathrm{~nm}$ high Si fins with different widths. This topography could have an effect on the piezoelectric and/or ferroelectric properties of the thin PZT layers compared to planar structures. In the LDV measurements, a $1 \mathrm{~V}$ dc and a $1 \mathrm{~V}$ sinusoidal ac-voltage at $8 \mathrm{kHz}$ frequency were applied to a $100 \mathrm{~nm}$ thick PZT layer. The response under these measurement conditions is shown in Figs. 5.7 (a) and (b) for FinFETs with $30 \mathrm{~nm}$ and a $1000 \mathrm{~nm}$ fin width, respectively. Figs. 5.7 (c) and (d) show a line scan of the piezoelectric displacement across the dotted lines in Figs. 5.7 (a) and (b), respectively. 

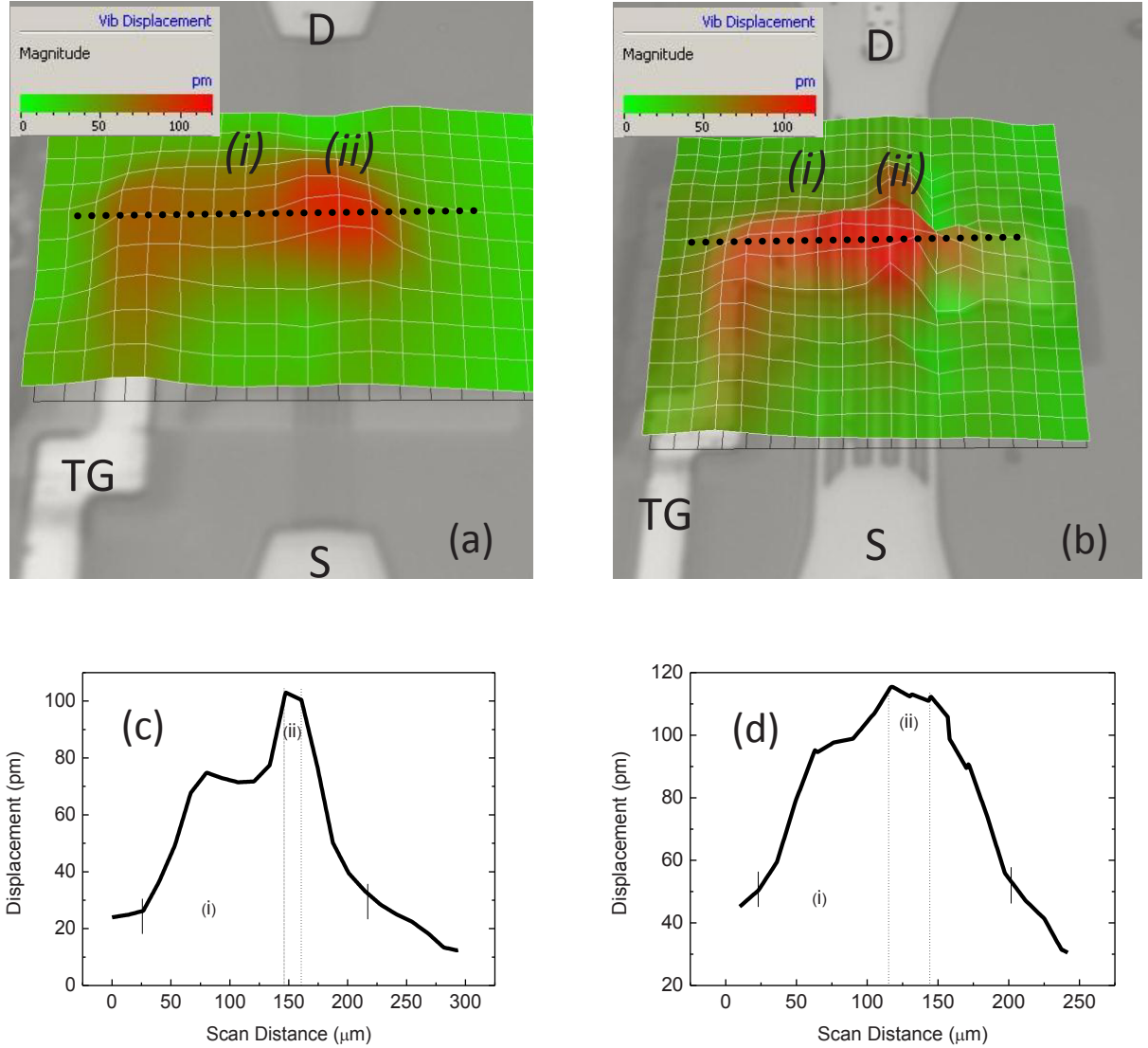

Fig. 5.7 2D upward response scans of a 5-fin PiezoFET with a (a) $30 \mathrm{~nm}$ and (b) $1000 \mathrm{~nm}$ fin width and $L_{\text {pigate }}=10 \mu \mathrm{m}, L_{\text {gate }}=30 \mu \mathrm{m}$. Nominally $1 \mathrm{~V}$ dc and a $1 \mathrm{~V}$ ac voltage at $8 \mathrm{kHz}$ frequency were applied to the film. The graphs in (c) and (d) are the line scan profiles of the displacement across the dotted line in (a) and (b). The channel response is indicated in between two vertical dotted lines. A $10 \mathrm{~nm}$ LNO+100 nm PZT film stack was used in this measurement.

The area where there is PZT between the TG and BG shows a clear displacement (Figs. 5.7 (a) and (b)). This is a strong indication of a piezoelectric effect. The displacement is enhanced on the FinFET channel. A piezoelectric coefficient of about $d_{33, f}=100-110 \mathrm{pm} / \mathrm{V}$ on the fin area and about $d_{33, \mathrm{f}}=80-90 \mathrm{pm} / \mathrm{V}$ outside the fin area under the top contact has been determined. These values are obtained under a nominal voltage (1 V) applied over the PZT layer. As will be shown in section 5.4.1, the actual voltage drop over the PZT layer is less than the applied voltage. The values are doubled when the applied nominal AC voltage was increased to $2 \mathrm{~V}$. This is an indication that the obtained displacement values are real displacement values.

Various explanations are possible to understand a higher displacement on the FinFET channel: $i$ ) thermal actuation as a result of increase in temperature due to high leakage 
between the TG and BG (the cause of this high leakage will be explained in section 5.4.1.1), ii) transverse stress related local bending resulting in an amplitude increase, and iii) topography of the surface due to the fin height. Furthermore, there is a $10 \%$ difference in the maximum displacement between the wide fin and narrow fin device. The maximum displacement is enhanced on wide fins possibly due to the relatively wide fin top surface, which is in the order of beam spot size and helps to obtain more signal from the fin top surface.

PiezoFETs with a $180 \mathrm{~nm}$ thick aluminum nitride (AIN) layer were also processed and an LDV analysis was performed on these devices. Piezoelectric activity was only observed for the $1000 \mathrm{~nm}$ wide devices. From the displacement data, $d_{33, \mathrm{f}}$ value of $13 \mathrm{pm} / \mathrm{V}$ is calculated. Bulk AIN has a value around $d_{33} \approx 5 \mathrm{pm} / \mathrm{V}[6,7]$. It shows that a higher displacement value than bulk value of AIN was obtained on the channel region. This is attributed to the mentioned reasons before.

\subsubsection{Polarization hysteresis measurement}

The polarization hysteresis $(P-E)$ loop measurements of the PZT layer on a planar structure and on a FinFET were done using the same setup as described in chapter 3 . The layers were also deposited under the same conditions as described in chapter 3. However, this time we could not obtain high polarization values on a planar structure for $100 \mathrm{~nm}$ thick PZT films, as shown in Fig. 5.8.

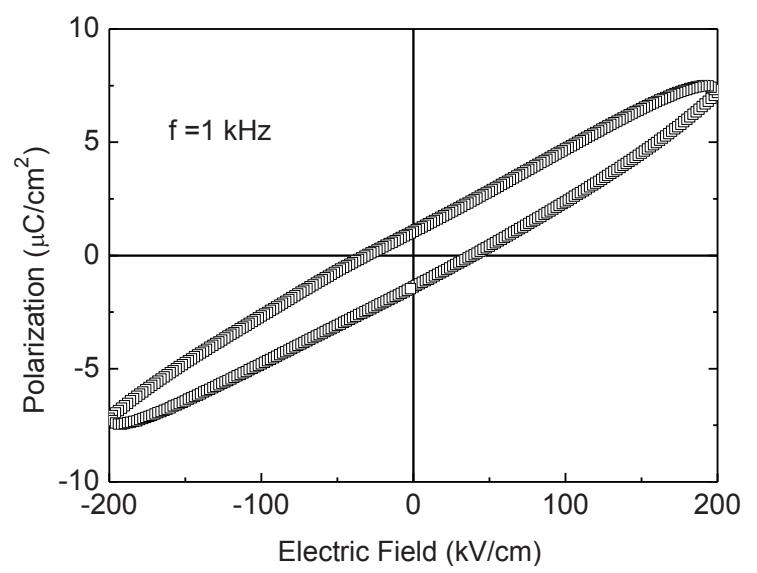

Fig. 5.8 Polarization hysteresis $(P-E)$ loops of a planar PZT capacitor with a $50 \mu \mathrm{m} \times 50 \mu \mathrm{m}$ area. The thickness of the PZT is $100 \mathrm{~nm}$. The experiment was performed at the applied ac-electric field of $\pm 200 \mathrm{kV} / \mathrm{cm}$ and $1 \mathrm{kHz}$ frequency. The maximum applied dc bias was $2 \mathrm{~V}$ with the bottom gate grounded. 
When the field was increased further, breakdown occurred. The leakage current was even higher in the non-planar structures (on FinFETs) and consequently no $P$-E loops could be obtained. The reason why the PZT layer on a planar surface has a higher leakage than the ones discussed in chapter 3 is probably due to (unintentionally) changed deposition conditions. Due to the relatively thin PZT layer at the fin edges, as shown in Figs. 5.6 (b) and (e), the electric field is higher and this results in higher leakage for the FinFET structures. We nevertheless expect that the PZT acts as a stressor because of the high displacement shown in section 5.3.2.

\subsection{Electrical characterization of the PiezoFET}

Studying the strain in PiezoFETs with optical strain characterization methods like Raman spectroscopy is a challenge. First, the Si channel will be strained when biasing the PZT layer. Therefore, the device needs to be biased during the optical analysis. Second, the PZT layer and the metallic layer on the PZT layer would strongly interfere with the laser signal.

The change in the band gap due to strain can be observed by the $I-V$ characteristics of the device. In addition, the mobility can be calculated from the $I-V$ curves. Since the change in mobility under stress is dependent on the stress level and the direction [4], any stress related effect can be recognized by electrical measurements. Therefore, I-V measurements were performed on PiezoFETs to recognize the effect of stress on the channels. The measurements were repeated using several devices with the same design parameters and it was found that they are reproducible.

\subsubsection{Effect of PZT deposition on the $I_{\mathrm{d}}-V_{\mathrm{gs}}$ curves}

Single fin and 5-fin PiezoFET devices for different $W_{\text {FIN }}$ were measured and analyzed. The schematic equivalent circuit is shown in Fig. 5.9. In the following discussion, a negative field over the PZT layer implies that a lower potential is applied to the top gate with respect to the bottom gate. All presented devices have a $100 \mathrm{~nm}$ thick PZT layer. 


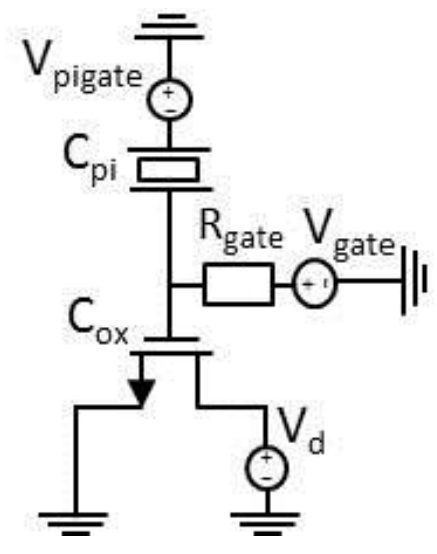

Fig. 5.9 Schematic equivalent circuit utilized for PiezoFET I-V measurements. To take into account its electro-mechanical properties, the piezoelectric capacitor $C_{p i}$ is represented by an acoustic resonator symbol.

Fig. 5.10 shows the $I-V$ curves of $100 \mathrm{~nm}$ wide fin devices before and after the $100 \mathrm{~nm}$ thick PZT deposition. A negative shift of threshold voltage $\left(\Delta V_{\text {th }}\right)$ as well as higher off current $\left(I_{\text {off }}\right)$ is observed after PZT deposition. This voltage shift is observed in all of the devices. The largest shift is observed for the $1000 \mathrm{~nm}$ wide devices. Almost no degradation in the transistor characteristics is observed after deposition and processing of the LNO/PZT layers. The observed shift in the I-V curve could be due to stress in Si fin after the high temperature $\left(650^{\circ} \mathrm{C}\right)$ LNO/PZT PLD process.

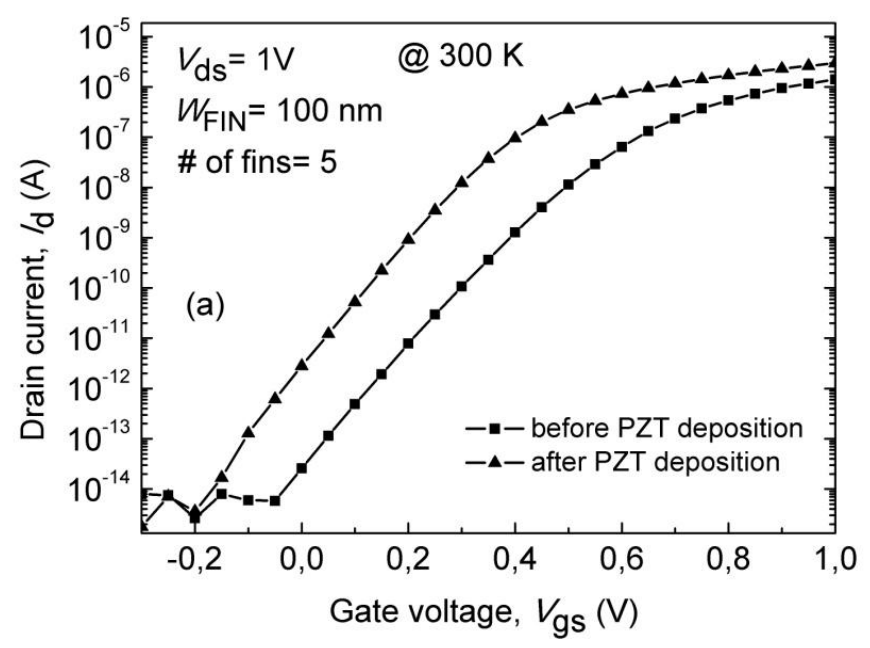




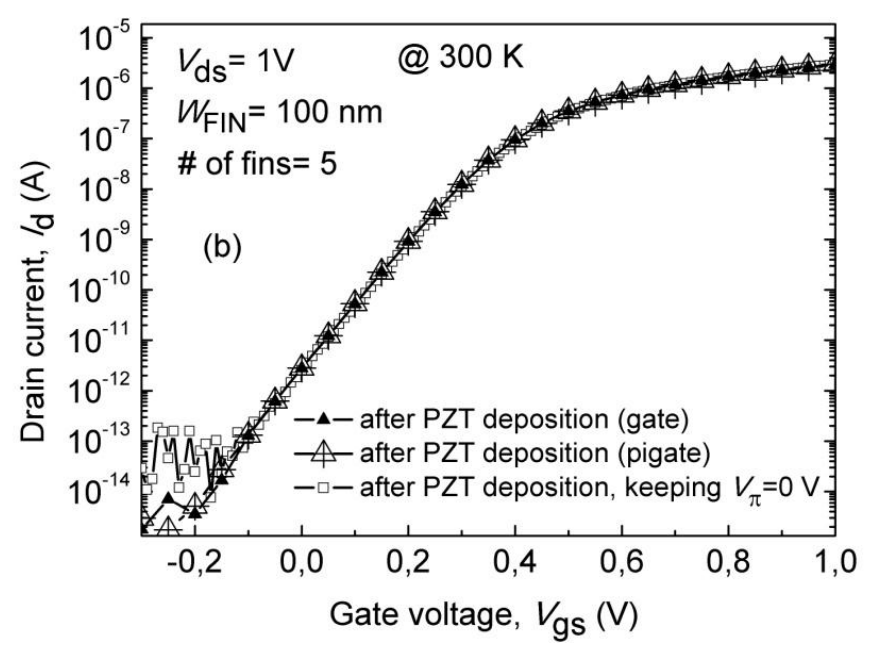

Fig. $5.10 I_{d}-V_{g s}$ device characteristics of a 5-fin devices with $L_{\text {pigate }}=10 \mu \mathrm{m}, L_{\text {gate }}=30 \mu \mathrm{m}, W_{\text {FIN }}=100$ $\mathrm{nm}$ at $V_{\mathrm{ds}}=1 \mathrm{~V}$ and room temperature (a) FinFET before (closed squares) and after PZT deposition (closed triangles), (b) FinFET after PZT deposition (closed triangles), PiezoFET biasing the top gate, keeping the bottom gate floating (open triangles) and the same gate bas on the top and bottom gate (open squares).

It has been reported before that due to the difference in coefficient of thermal expansion (CTE) of the Si and PZT; a thermal stress can be developed in the PZT film [8]. Since Si has a CTE of $2.6 \times 10^{-6} \mathrm{~K}^{-1}$ [9] and PZT has a CTE of $6.0 \times 10^{-6} \mathrm{~K}^{-1}$ [10], additional thermal biaxial in-plane tensile stress is formed in the PZT film during the cooling down process from deposition temperature $\left(650{ }^{\circ} \mathrm{C}\right)$ to room temperature. This will result in biaxial compressive stress in Si. Thermal expansion related stress profile and analysis has been reported before $[11,12]$.

The shift cannot be explained with the strain effect in the Si only since the observed shift of around $0.15 \mathrm{~V}$ is very large. Other possible reasons for this shift will be discussed in section 5.4.2.

Another important observation is that the SS is almost unchanged/only slightly reduced after the PZT deposition (which is more clearly observed in Fig. 5.17). More explanation on this subject will be given in section 5.4.2.

Fig. 5.10 (b) shows that the same $I_{d}-V_{g s}$ curves are obtained irrespective when only the top gate (or pigate), or only the bottom gate is biased or when the piezoelectric layer is biased at zero volt. This means that there is almost no voltage drop over the PZT layer. To calculate the ratio of the voltage drop, the value of $\varepsilon / t$ for the oxide and PZT layer has to be known. In chapter $3, \varepsilon_{\mathrm{PZT}}$ was determined being 600 at zero volt. Therefore, $\varepsilon_{\text {ox }} / t_{\text {ox }}$ is 11 times higher than that of $\varepsilon_{P Z T} / t_{P Z T}$. Hence, the voltage drop over the PZT layer is 11 times 
less than that over the oxide. Therefore, we expect no difference in the $I_{d}-V_{g s}$ characteristics of the device when operating with one gate only and leave the other one floating.

Furthermore, unlike reported for FerroFETs [13], no hysteresis behavior in our PiezoFETs is observed. Commonly, ferroelectric materials show hysteresis due to domain wall movements [14]. However, in our case, the relatively high leakage current interferes with the polarization switching and results in a non-hysteric behavior.

\subsubsection{Effect of PZT biasing on the $I_{\mathrm{d}}-V_{\mathrm{gs}}$ curves}

In our further analysis, the PZT layer was biased at a constant and changing net bias between the top and (bottom) gate $\left(V_{\mathrm{BG}}=V_{\mathrm{g}}\right)$ to observe the converse piezoelectric effect on the operation of the device (Fig. 5.11). The schematic of the measurement setup is shown in Fig. 5.9.
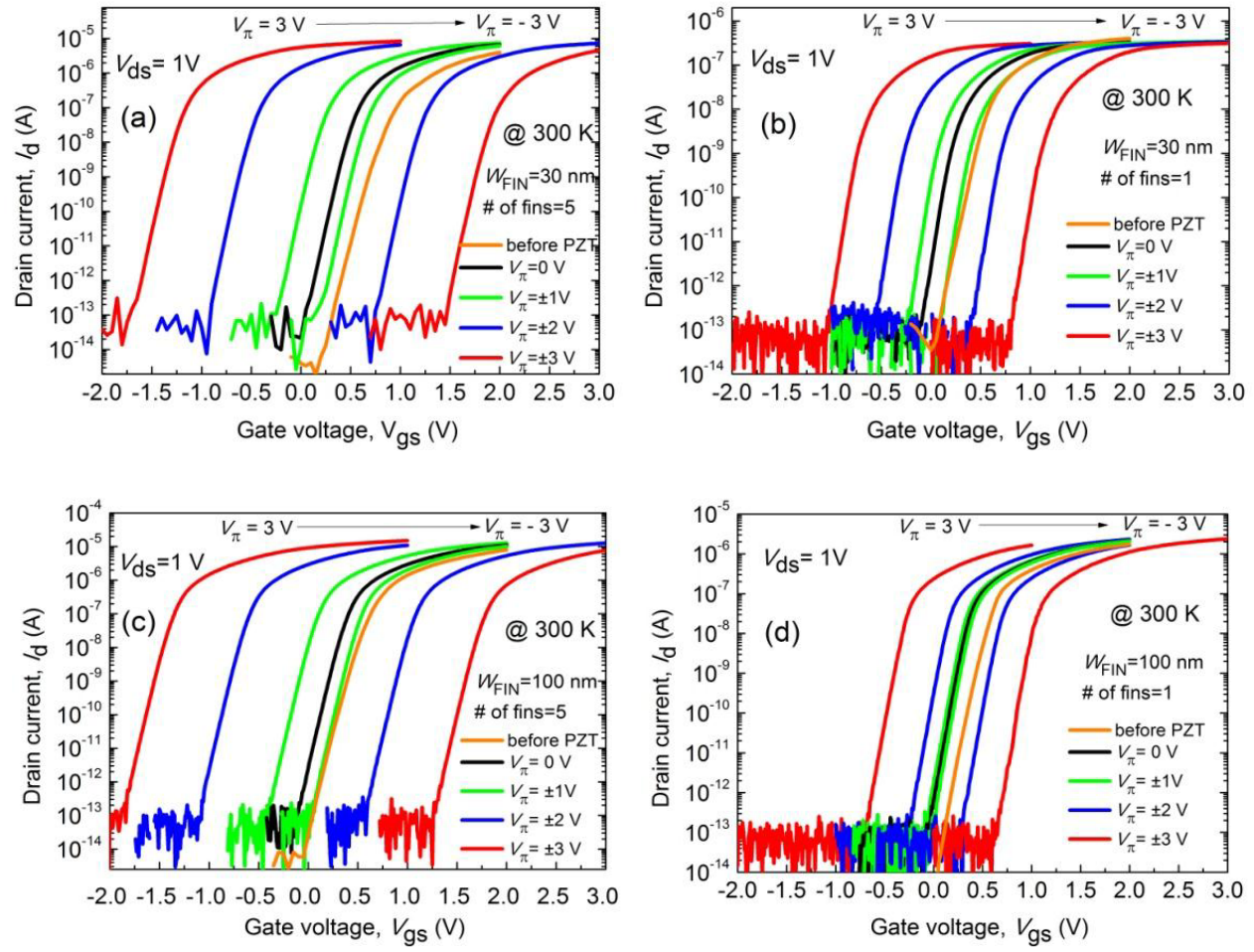

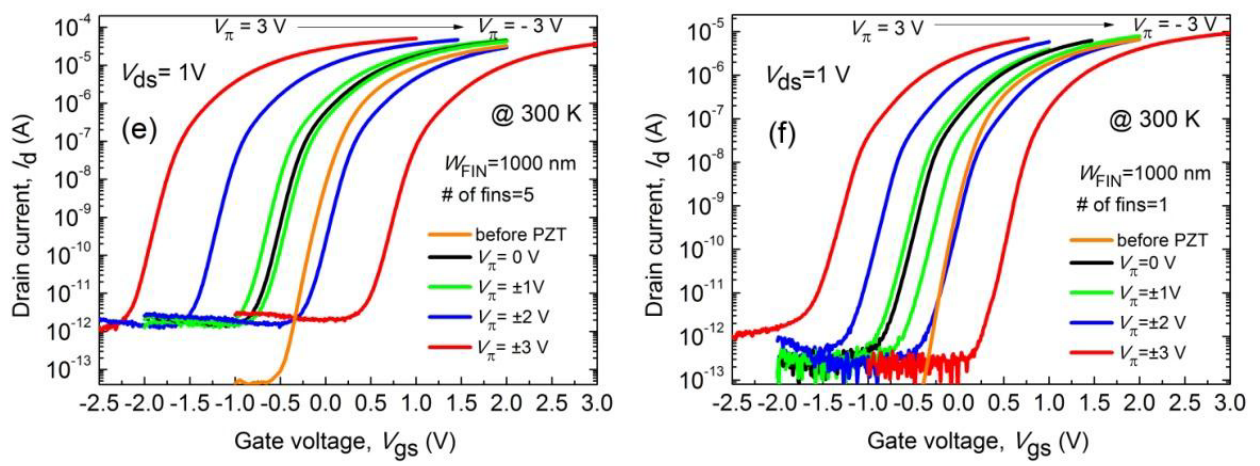

Fig. $5.11 I_{d}-V_{g s}$ device characteristics of the PiezoFETs for different $V_{\pi}$ with (a) 5 -fins with $W_{\mathrm{FIN}}=30$ $\mathrm{nm}$, (b) single fin with $W_{\text {FIN }}=30 \mathrm{~nm}$, (c) 5-fins with $W_{\text {FIN }}=100 \mathrm{~nm}$, (d) single fin with $W_{\text {FIN }}=100 \mathrm{~nm}$, (e) 5-fins with $W_{\text {FIN }}=1000 \mathrm{~nm}$, (f) single fin with $W_{\mathrm{FIN}}=1000 \mathrm{~nm}$. All devices have $L_{\mathrm{BG}}=30 \mu \mathrm{m}$ and $L_{\mathrm{TG}}=10$ $\mu \mathrm{m}$. The measurements were performed at room temperature.

First, a constant net bias was applied to the PZT layer and the device was measured by sweeping the bottom gate voltage. Figs. 5.11 (a) and (b) show the $I_{d}-V_{g s}$ curves of $30 \mathrm{~nm}$ wide 5 -fin and single fin PiezoFETs under $0 \mathrm{~V}, \pm 1 \mathrm{~V}, \pm 2 \mathrm{~V}$ and $\pm 3 \mathrm{~V}$ constant net bias across the PZT layer $\left(V_{\pi}\right)$. For zero bias over the PZT layer, a large negative shift is observed for both devices. A negative shift is also observed under positive bias whereas a positive shift is observed under negative bias. The threshold voltage shift is increased when $V_{\pi}$ is increased. However, single fin devices show a smaller shift (see also Fig. 5.14). Figs. 5.11 (c)-(f) present the similar measurements of 5-fin and single fin device structures with 100 $\mathrm{nm}$ and $1000 \mathrm{~nm}$ fin widths. Note that, to check the reproducibility of this effect, another wafer was processed and the same PiezoFETs were measured. We observed a similar behavior for these devices as well.

These devices can be utilized to combine low $V_{t h}$, low $I_{\text {off }}$ and steep sub-threshold swing (SS). In order to do so, $I_{d}-V_{g s}$ device characteristics can be measured under an increasing net bias over the PZT layer In Fig. $5.12 I_{d}-V_{g s}$ curves of a 5-fin device with $W_{\text {FIN }}=30 \mathrm{~nm}$ are plotted at changing $V_{\pi}$ from $0 \mathrm{~V}$ to $1 \mathrm{~V}, 2 \mathrm{~V}$ and $3 \mathrm{~V}$ during the gate sweeping. By increasing the $V_{\pi}$ from $0 \mathrm{~V}$ to $3 \mathrm{~V}$, the $S S$ was decreased from $74 \mathrm{mV} / \mathrm{dec}$ to $48 \mathrm{mV} / \mathrm{dec}$. Therefore, with the help of the threshold voltage shift effect and by tuning $V_{\pi}$, a low SS can be obtained. Although there is a power drawback due to a need for $V_{\mathrm{TG}}>V_{\mathrm{gs}}+1$ and the leakage current, this figure shows that SS can be modified by controlling the gate biases. 


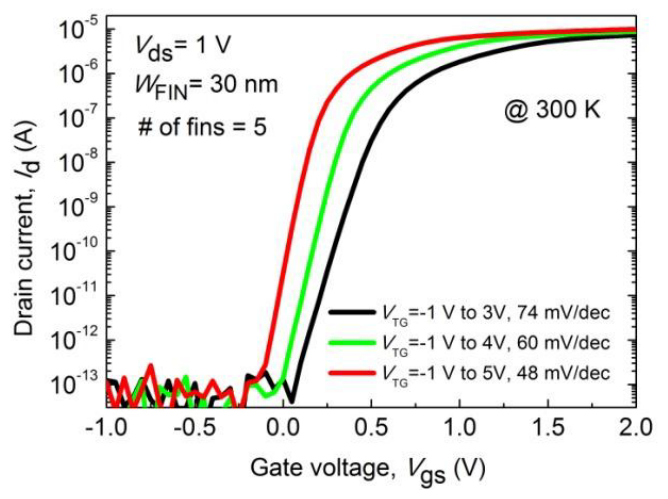

Fig. $5.12 I_{d}-V_{g s}$ device characteristics of a 5 -fin, $W_{\mathrm{FIN}}=30 \mathrm{~nm}$ device at $V_{\mathrm{TG}}$ changing from $-1 \mathrm{~V}$ to $5 \mathrm{~V}$ while $V_{\mathrm{gs}}\left(=V_{\mathrm{BG}}\right)$ is sweeping from $-1 \mathrm{~V}$ to $2 \mathrm{~V}$. Device have $L_{\mathrm{BG}}=30 \mu \mathrm{m}$ and $L_{\mathrm{TG}}=10 \mu \mathrm{m}$. The measurements were performed at room temperature.

Fig. 5.13 shows the $\Delta V_{\text {th }}-V_{\pi}$ curves for the devices presented in Fig. 5.11. There is an exponential dependency of the $\Delta V_{\text {th }}$ on the applied bias, reaching $+1.5 \mathrm{~V}$ and $-2 \mathrm{~V}$ under -3 $\mathrm{V}$ and $+3 \mathrm{~V}$ applied bias over PZT, respectively. There is also an asymmetry regarding the polarity. In addition, as mentioned before, 5-fin devices show a larger shift than 1-fin devices. For a single fin device, the largest shift seems to occur for the $30 \mathrm{~nm}$ fin width. A device with a shorter gate length is also plotted in the same figure to show that there is small dependency of $\Delta V_{\text {th }}$ with the bottom gate length.

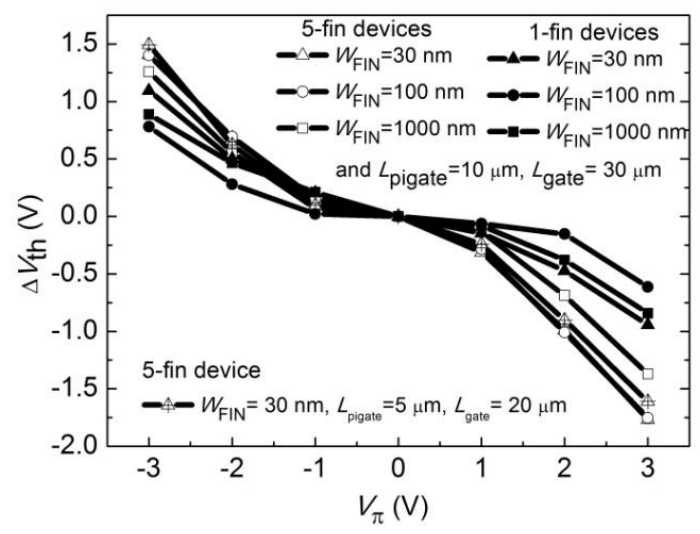

Fig. $5.13 \Delta V_{\mathrm{th}}-V_{\pi}$ curve of 5-fin and single fin PiezoFET devices with a $30 \mathrm{~nm}, 100 \mathrm{~nm}$ and $1000 \mathrm{~nm}$ fin width. Devices have $L_{\mathrm{BG}}=30 \mu \mathrm{m}$ and $L_{\mathrm{BG}}=10 \mu \mathrm{m}$, except for one device with $L_{\mathrm{BG}}=20 \mu \mathrm{m}$ and $L_{\mathrm{TG}}=$ $5 \mu \mathrm{m}$. 
In Fig. 5.14 the $J-V_{\pi}$ characteristics of the PZT capacitor on devices with different fin widths as well as on a planar surface are plotted.

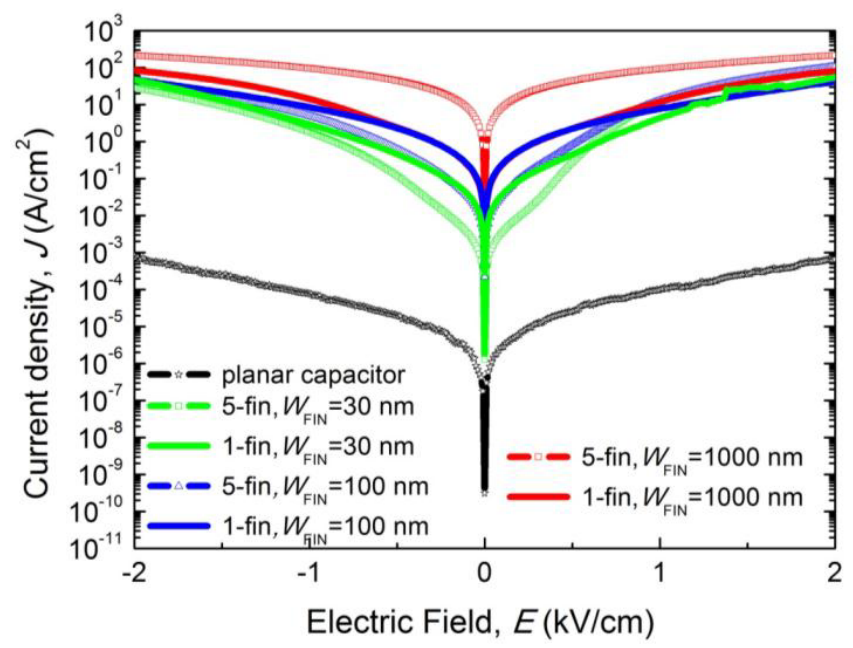

Fig. $5.14 J-V_{\pi}$ characteristics of the PZT capacitor on a planar surface and on 5-fin and single fin FinFETs. $L_{T G}=10 \mu \mathrm{m}$ for all devices.

The leakage current of the PZT and the $\Delta V_{\text {th }}$ (Fig. 5.13) of the PiezoFET show an exponential dependency on the applied bias. The leakage current of the PZT capacitor is high on planar surfaces compared to that obtained for the planar PZT capacitors discussed in chapter 3 . The leakage current is, however, much higher when integrated with FinFETs. Typically, the leakage currents show a symmetric behavior similar to those of the planar structures as discussed in chapter 3. In general, single fin devices show less leakage than 5-fin devices above certain fields. However, the leakage current does not scale linearly with the number of fins. The main issue remains to be the step coverage of the PZT layer around the fins.

PiezoFETs with a $180 \mathrm{~nm}$ thick AIN layer were deposited and measured for comparison. Figs. 5.15 (a)-(c) show the $I_{d}-V_{g s}$ device characteristics of the AIN PiezoFET with a $180 \mathrm{~nm}$ thick AIN layer. 

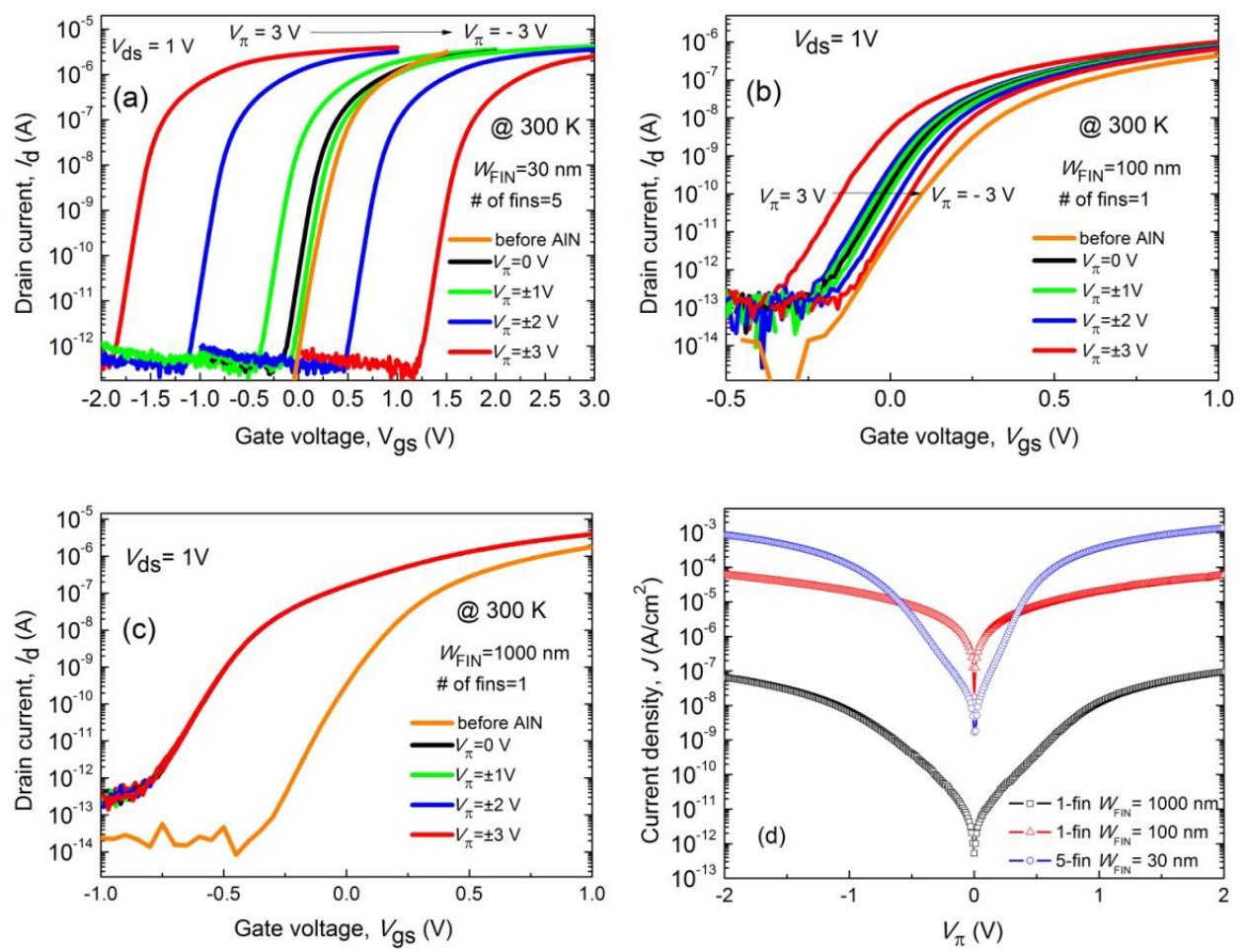

Fig. $5.15 I_{d}-V_{g s}$ device characteristics of the AIN PiezoFETs for different $V_{\pi}$ with (a) 5 -fins with $W_{\text {FIN }}=$ $30 \mathrm{~nm}$, (b) single fin with $W_{\mathrm{FIN}}=100 \mathrm{~nm}$ and (c) single fin with $W_{\mathrm{FIN}}=1000 \mathrm{~nm}$. The leakage current density of AIN is plotted in (d) corresponding to devices plotted in (a)-(c). All devices have $L_{B G}=30$ $\mu \mathrm{m}$ and $L_{T G}=10 \mu \mathrm{m}$. The measurements were performed at room temperature.

Similar to the PZT deposited devices, a negative shift in the threshold voltage is observed after AIN deposition without any bias. The amount of shift is smaller compared to devices with PZT layer in Fig. 5.11. The strain formed due to the difference in CTE is regarded as the reason for this shift. The CTE of AIN is smaller than PZT and the deposition temperature is lower, i.e. lower strain is expected. Again, the largest shift occurs for the $1000 \mathrm{~nm}$ wide device.

The AIN PiezoFET with 5-fins of $30 \mathrm{~nm}$ fin width shows the same amount of shift under applied bias compared to a PZT PiezoFET with the same dimensions (see Fig. 5.11 (a)). However, for a fin width of $100 \mathrm{~nm}$ (Fig. 5.15 (b)), the shift is smaller for the AIN PiezoFET. When the fin width is $1000 \mathrm{~nm}$ (Fig. 5.15 (c)), no shift is observed. The measured leakage current of AIN on devices is given Fig. 5.15 (d). In this case, a single fin $1000 \mathrm{~nm}$ wide device has five orders of magnitude lower leakage current than a $30 \mathrm{~nm}$ wide 5-fin device at $2 \mathrm{~V}$. The shift proportionally decreases with the leakage current and this is a clear indication that the threshold voltage shift is related to the leakage current through the piezoelectric layer, which is most probably higher at the fin sidewalls. 
This threshold voltage shift can be explained by an electrical DC equivalent circuit diagram of a leaky piezoelectric capacitor on top of the FET connected by the (bottom) gate resistance. $\mathrm{A} \Delta V_{\mathrm{th}}$ is expected when the bottom gate resistance is comparable with the resistance of the piezoelectric layer. The corresponding schematic equivalent circuit is shown in Fig. 5.16.

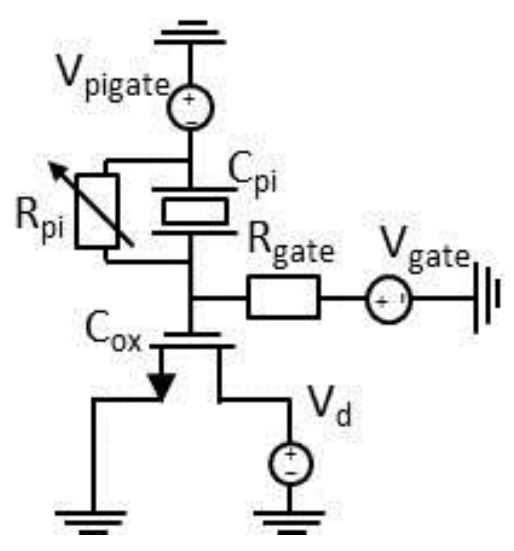

Fig. 5.16 Modified schematic equivalent circuit of the measurement setup utilized for the PiezoFET I-V measurements.

In the ideal case (Fig. 5.9), there is no current through the piezoelectric capacitor $C_{\mathrm{pi}}$ and the surface potential on the gate is $V_{\text {gate. }}$ However, when there is a (variable) resistor $\left(R_{\text {pi }}\right)$ parallel to the piezoelectric capacitor (Fig. 5.16), the surface potential will be influenced. The influence of $R_{\mathrm{pi}}$ on the surface potential is determined by the relative values of $R_{\text {gate }}$ and $R_{\mathrm{pi}}$.

The resistivity and thickness of the TiN external connection to the bottom gate is $160 \mu \Omega$ $\mathrm{cm}$ and $6 \mathrm{~nm}$, respectively (see chapters 3 and 4). The length of this connection is $50 \mu \mathrm{m}$ and the width is $30 \mu \mathrm{m}$. This yields a resistance of $R_{\text {gate }}=445 \Omega$. The leakage current of the PZT capacitor deposited on the $1000 \mathrm{~nm}$ wide single fin device was $2.5 \mathrm{~mA}$ at $V_{\pi}=3 \mathrm{~V}$. Therefore, by calculating the real (internal) voltage drop over the PZT layer, we obtain a PZT resistance value of about $755 \Omega$.

According to the calculated values of resistances, $\approx 2 / 3 \cdot V_{\pi}$ has dropped across the PZT layer whereas the rest of the voltage drop is across the gate. The surface potential is increased by this amount and therefore, a threshold voltage shift occurs. It is observed in Fig. 5.13 that under a $3 \mathrm{~V}$ bias, the threshold shift is about $0.9 \mathrm{~V}$ for $1000 \mathrm{~nm}$ wide single fin device. This value is $1.11 \mathrm{~V}$, very close to the calculated voltage drop over the gate. Since we do not account for distribution effects caused by current spreading on top of the channel region, the calculated resistance value is an approximation. On the other hand, the $1000 \mathrm{~nm}$ fin width device with the AIN layer shows low leakage (Figs. 5.15 (c) and (d)), the $R_{\mathrm{pi}}$ is high and the surface potential is less affected. 
In summary, the observed threshold voltage shift in our devices is a result of the piezoelectric layer leakage current. This section will continue with the PZT deposited PiezoFETs.

To observe the effect of $V_{\pi}\left(=\mathrm{V}_{\mathrm{TG}}-\mathrm{V}_{\mathrm{BG}}\right)$ on the sub-threshold region, SS- $V_{\pi}$ graphs of PZT deposited PiezoFETs are plotted in Figs. 5.17 (a) and (b).
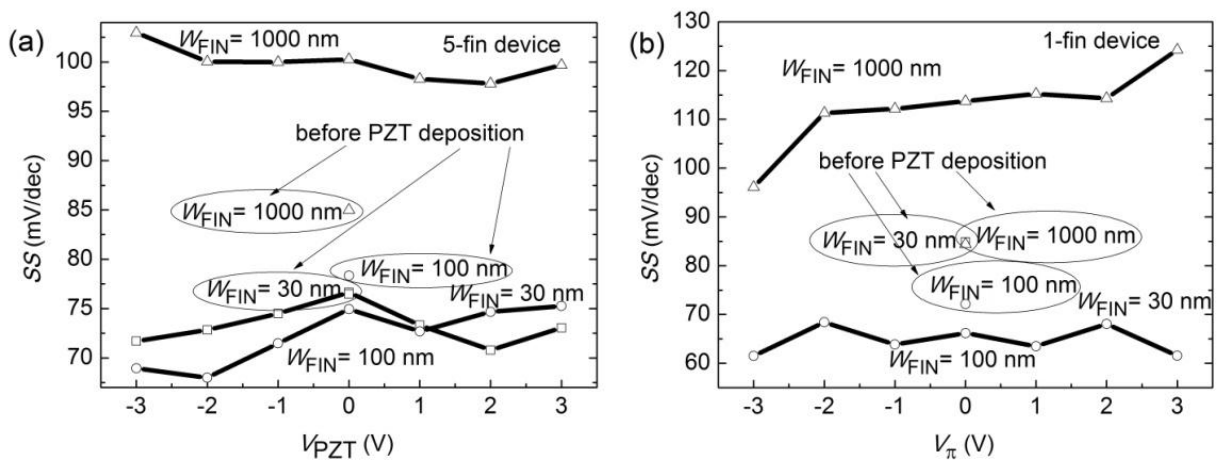

Fig. $5.17 \mathrm{SS}-V_{\pi}$ graph of (a) 5-fin, (b) 1 -fin devices with $W_{\mathrm{FIN}}=30,100$ and $1000 \mathrm{~nm}$ at $V_{\mathrm{ds}}=1 \mathrm{~V}$. All devices have $L_{B G}=30 \mu \mathrm{m}$ and $L_{T G}=10 \mu \mathrm{m}$.

The SS values are above $60 \mathrm{mV} / \mathrm{dec}$ indicating presence of interface traps, in particular for the narrow fin devices. Fig. 5.17 also shows that the SS is decreased (reduced effective interface trapping of charge carriers) for devices with $30 \mathrm{~nm}$ and $100 \mathrm{~nm}$ fin width after PZT deposition and without biasing. Up to $20 \%$ improvement in the SS is observed after the PZT deposition. It could indicate that the gate oxide $\left(\mathrm{SiO}_{2}\right)$ layer has been stressed along its interface with the Si body, possibly affecting the band alignment. The effect of fixed strain on the trapping probability and band modulation was reported before [15, 16]. This work reports it on our tunable strain devices as well.

Stress in the oxide layer can be a reason for the slightly reduced SS and possibly a part of the observed threshold voltage shift. Since the $\mathrm{SiO}_{2}$ is less stiff than $\mathrm{Si}$, most of the strain will be absorbed in the oxide. For instance, the FEM simulations of the NXP FinFETs discussed in chapter 2 predict higher strain values in the gate dielectric than in the Si body (Fig. 2.3).

For the $1000 \mathrm{~nm}$ wide fin sample, however, SS increased. This is possibly due to its different crystal orientation. Wide fin devices have a (001) surface orientation, instead of the $(1 \overline{1} 0)$ surface sidewall orientation dominant for narrow fin samples. The available bonds for (001) surface is smaller than $(1 \overline{1} 0)$ surface. That would change the density of interface states. In addition, as will be mentioned later in section 5.4.3.1, we expect a different stress configuration for wide and narrow fins. This would have a different effect on the band alignment. 
The reduction of the SS for narrow fins indicates a decrease of the interface trap density. For wide fins, however, the SS increases and this indicates an increase in the interface trap density (Table 5.3). Furthermore, the $S S$ is modified slightly (up to $10 \%$ ) by biasing the PZT layer on the 5 -fin devices. For a 5 -fin device with $30 \mathrm{~nm}$ and $100 \mathrm{~nm}$ fin width, the SS is slightly decreased with an applied PZT bias (Fig. 5.17 (a)). There is almost no trend in SS for single fin devices with the same fin width (Fig. 5.17 (b)).

The interface trap density, $D_{\mathrm{it}}$, has been calculated as described in section 4.2.3. Table 5.3 shows the calculated $D_{\text {it }}$ values for the 5 -fin devices presented in Fig. 5.17 (a). For $30 \mathrm{~nm}$ and $100 \mathrm{~nm}$ wide fins, biasing the PZT decreases the trap density. The $D_{\mathrm{it}}$ values under PZT bias are not given for $1000 \mathrm{~nm}$ wide fins due to the interference of high leakage current due to short channel effects.

Table 5.3 $D_{\text {it }}$ values calculated from the minimum SS values in Fig. 5.17 (a).

\begin{tabular}{ccccc}
\hline \hline $\begin{array}{c}W_{F I N} \\
(\mathrm{~nm})\end{array}$ & $\begin{array}{c}D_{i t} \text { before PZT } \\
\left(\mathbf{1} / \mathrm{eV}-\mathrm{cm}^{2}\right)\end{array}$ & $\begin{array}{c}D_{i t} \text { at } V_{\pi}=\mathbf{0 ~ V} \\
\left(\mathbf{1} / \mathrm{eV}-\mathrm{cm}^{2}\right)\end{array}$ & $\begin{array}{c}D_{i t} \text { at } V_{\pi}=3 \mathbf{V} \\
\left(\mathbf{1} / \mathrm{eV}-\mathrm{cm}^{2}\right)\end{array}$ & $\begin{array}{c}D_{i t} \text { at } V_{\pi}=-\mathbf{3} \mathrm{V} \\
\left(\mathbf{1} / \mathrm{eV}-\mathrm{cm}^{2}\right)\end{array}$ \\
\hline \hline 30 & $5.23 \times 10^{11}$ & $5.37 \times 10^{11}$ & $4.21 \times 10^{11}$ & $3.78 \times 10^{11}$ \\
100 & $5.92 \times 10^{11}$ & $4.82 \times 10^{11}$ & $4.93 \times 10^{11}$ & $2.88 \times 10^{11}$ \\
\hline \hline
\end{tabular}

Since a high piezoelectric response was obtained on top of the device structures (section 5.3.3), a certain amount of strain is expected in the underlying layers. Changing $V_{\pi}$, i.e. strain, will change the mobility. The following section discusses the effect of strain on the mobility in FinFET devices. The threshold shift has been taken into account in mobility extraction. Therefore, it is expected that it does not affect the obtained results.

\subsubsection{Mobility calculations}

The effective electron mobility of the PiezoFET has been calculated in the same way as described in chapter 4 and hence the effect of the $\Delta V_{t h}$ has already been compensated for. Fig. 5.18 shows the effective electron mobility as a function of the electric field ( $\left.\mu_{\text {eff }}-E\right)$ for 5 -fin and single fin $(1 \overline{1} 0) /[110]$ PiezoFET devices with $W_{\mathrm{FIN}}=30 \mathrm{~nm}, 100 \mathrm{~nm}$ and 1000 $\mathrm{nm}$ for different $V_{\pi}$. For comparison, the $\mu_{\text {eff }} E$ curves of the (100) bulk MOSFET (Fig. 5.18 (a)) and the (110) FinFET and the (110) bulk MOSFET (Fig. 5.18 (e)) are also shown [17, 18]. 

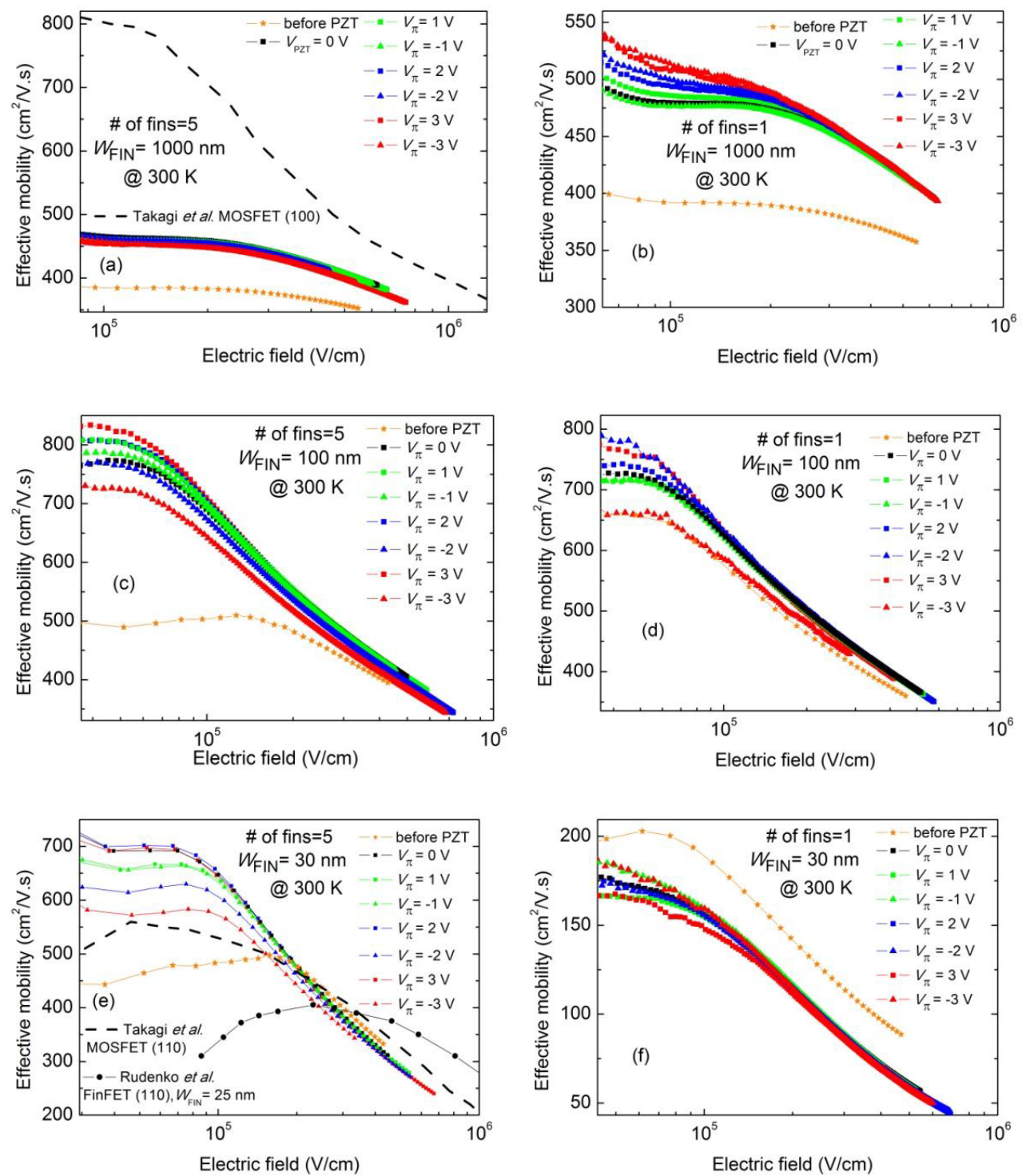

Fig. 5.18 Effective electron mobility-electric field curves of PiezoFETs for different biasing over the PZT layer with (a) 5-fins of $W_{\mathrm{FIN}}=1000 \mathrm{~nm}$ and (100) MOSFET obtained from Takagi et al. [17], (b) a single fin of $W_{\mathrm{FIN}}=1000 \mathrm{~nm}$, (c) 5-fins of $W_{\mathrm{FIN}}=100 \mathrm{~nm}$, (d) a single fin of $W_{\mathrm{FIN}}=100 \mathrm{~nm}$, (e) 5-fins of $W_{\text {FIN }}=30 \mathrm{~nm}$ and (110) FinFET with $W_{\mathrm{FIN}}=25 \mathrm{~nm}$ obtained from Rudenko et al. [18], (110) MOSFET obtained from Takagi et al. [17], (f) a single fin of $W_{\mathrm{FIN}}=30 \mathrm{~nm}(T=300 \mathrm{~K})$. All devices have $L_{\mathrm{BG}}=30$ $\mu \mathrm{m}, L_{\mathrm{TG}}=10 \mu \mathrm{m}$. All measurements were performed at $300 \mathrm{~K}$.

Up to $\approx 20 \%$ increase in the mobility was observed after PZT deposition on $1000 \mathrm{~nm}$ wide 5-fin and single fin devices (Fig. 5.18 (a) and (b)). The effect on $100 \mathrm{~nm}$ and $30 \mathrm{~nm}$ wide 5fin devices at low fields is stronger; up to $\approx 50 \%$ increase in mobility (Fig. 5.18 (c) and (e)). For high fields, no significant change is observed. The single fin device with $100 \mathrm{~nm}$ fin 
width show up to $8 \%$ increase in mobility (Fig. 5.18 (d)). The mobility is decreased by about $12 \%$ for a single fin device $30 \mathrm{~nm}$ fin width (Fig. 5.18 (f)).

The change in the mobility, however, is smaller for varying $V_{\pi}$. The $\mu_{\text {eff }}$ is affected up to $\approx$ $20 \%$ by the voltage $V_{\pi}$ between the top and bottom gate. Compared to the mobility values given in literature [18], the low field mobility is higher whereas the high field mobility is lower in our devices. The higher mobility at lower fields is an indication of less effective Coulomb scattering (which has an effect on mobility in this region), most probably because of a higher fin and the use of $\mathrm{SiO}_{2}$ as a gate dielectric in our case. In [18], a high- $\kappa$ material has been used in which dipoles that are close to the channel could increase the Coulomb scattering. However, the lower mobility at higher fields is an indication of more surface imperfections in our devices.

The mobility in devices with deposited AIN was also calculated for comparison. An increase in mobility after AIN deposition was also observed. The most significant change is obtained again for $1000 \mathrm{~nm}$ wide devices. Biasing of the AIN layer showed no significant change in the mobility. This is expected due to a lower piezoelectric response of the AIN compared to that of PZT.

To show the trend in the mobility more clearly when biasing the 5-fin and single fin PZT PiezoFETs, the mobility- $V_{\pi}$ curve has been plotted when biased at moderate inversion (Fig. 5.19) and at strong inversion (Fig. 5.20). For the $30 \mathrm{~nm}$ and $100 \mathrm{~nm}$ wide fin devices, the applied fields are at $E=5 \times 10^{4} \mathrm{~V} / \mathrm{cm}$ and $E=2 \times 10^{5} \mathrm{~V} / \mathrm{cm}$ for moderate and strong inversion, respectively. Due to the fact that the $W_{\mathrm{FIN}}=1000 \mathrm{~nm}$ device act as a partially depleted SOI device, a higher electric field is needed to obtain strong inversion. Therefore, for a $1000 \mathrm{~nm}$ wide device, the effective mobility- $V_{\pi}$ curve is plotted at a higher field of $1 \times 10^{5} \mathrm{~V} / \mathrm{cm}$ and $4 \times 10^{5} \mathrm{~V} / \mathrm{cm}$ for moderate and strong inversion, respectively.
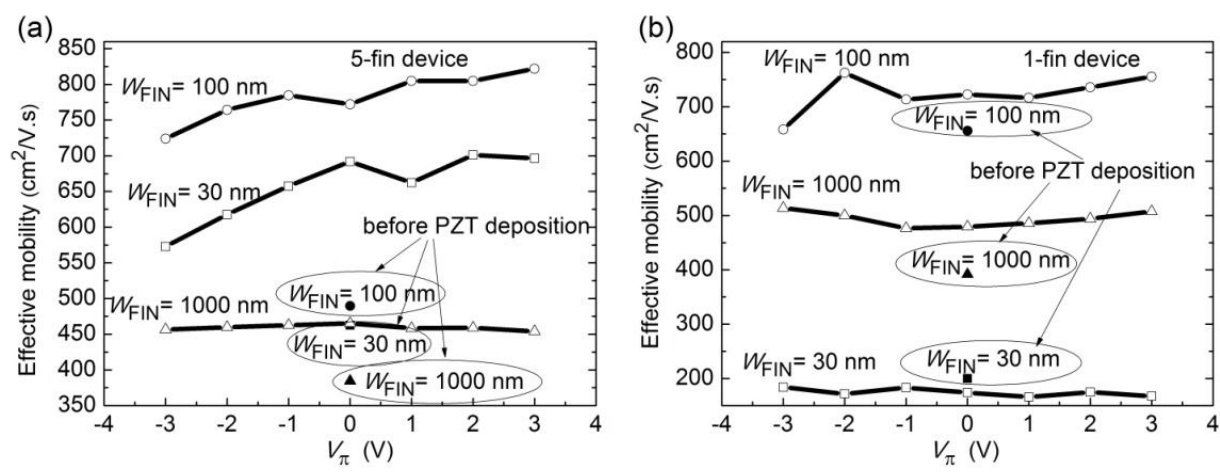

Fig. 5.19 Effective electron mobility- $V_{\pi}$ curves of devices at a $5 \times 10^{4} \mathrm{~V} / \mathrm{cm}$ field for $30 \mathrm{~nm}$ and 100 $\mathrm{nm}$ wide fin devices and at a $1 \times 10^{5} \mathrm{~V} / \mathrm{cm}$ field for a $1000 \mathrm{~nm}$ wide fin device for (a) 5 -fins and (b) a single fin. 
(a)

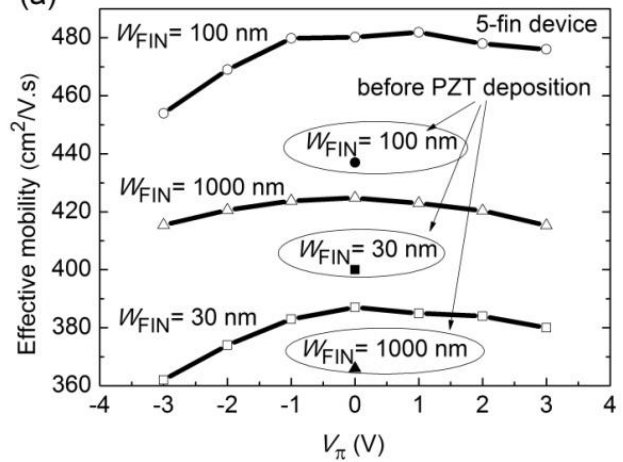

(b)

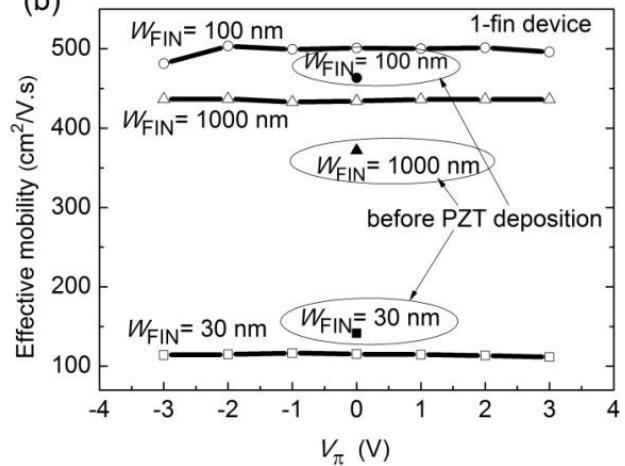

Fig. 5.20 Effective electron mobility- $V_{\pi}$ curve of devices at a $2 \times 10^{5} \mathrm{~V} / \mathrm{cm}$ field for $30 \mathrm{~nm}$ and $100 \mathrm{~nm}$ wide fin devices and at a $4 \times 10^{5} \mathrm{~V} / \mathrm{cm}$ field for a $1000 \mathrm{~nm}$ wide fin device for (a) 5-fins and (b) a single fin.

At low fields, the effective mobility shows an increasing trend under positive applied bias and decreasing trend under negative applied bias for $30 \mathrm{~nm}$ and $100 \mathrm{~nm}$ wide devices with 5 -fins. Single fin devices show no clear trend. At high fields, mobility decreased again under negative applied bias. But no trend is observed for positive applied bias. Single fin devices show no clear trend again. The next step is to discuss the possible reasons for the observed mobility behavior.

\subsubsection{Discussion}

First, the effect of the deposition of the PZT layer on the electron mobility will be discussed. Second, we discuss the effect of strain induced by the converse piezoelectric effect on the electron mobility. Before the discussion, several things should be kept in mind:

1) It should be emphasized that stress related effects do not impact the electron mobility in the same way for weak inversion and strong inversion [4]. The increase of mobility by strain will be enhanced by an increase of the relaxation time due to reduced intervalley scattering in weak inversion. This is because of the reduction in the amount of conduction band valleys, the so-called degeneracy, which contributes to the current. In addition, the electrons in the inversion layer are strongly confined. The quantization effect is known to counteract the stress induced repopulation of the electron valleys [5]. At strong inversion this confinement strongly depends on the applied bias contrary to weak inversion. Therefore, we observe a different behavior in the strong and moderate inversion regimes.

2) In addition to point 1), in the moderate inversion (i.e. near threshold voltage) regime Coulomb scattering is important for the mobility. This type of scattering is affected by ions present in the silicon body or at the interface, but by effective interface traps as 
well ( $\mu_{i c} \propto 1 / N_{i t}$, with $N_{\text {it }}$ is the amount of traps) [19]. For strong inversion, another non-ideal effect such as surface scattering is important: $\mu_{s r} \propto 1 /(\Delta L)^{2}$, with $\Delta$ is the surface deviation perpendicular to the surface flatness, and $L$ is the lateral length scale of the roughness fluctuations $[20,21]$. However, surface scattering is less important for narrow fins (see chapter 1 ).

3) As mentioned before, when we were illustrating the strain effect, we neglected the strain relieving effect of the intermediate layers between the PZT layer and the Si fins. Also, effects such as non-uniformity caused by poor step coverage of several layers along the fin perimeter were not taken into account. Either way these issues will not dramatically affect the conclusions drawn from this qualitative discussion.

\section{After deposition (before applied bias) of the PZT layer:}

As shown in Figs. 5.19 and 5.20, the mobility has increased for $1000 \mathrm{~nm}$ and $100 \mathrm{~nm}$ wide fin devices with 5 -fins and a single fin after the LNO/PZT deposition for $V_{\pi}=0 \mathrm{~V}$. The mobility has decreased for a $30 \mathrm{~nm}$ wide fin device with 5 -fins and a single fin for high fields (Figs. 5.20 (a) and (b)). Only the 5-fin device shows higher mobility values at lower field in Fig. 5.19 (a) (in the moderate inversion regime when Coulomb or ion scattering is important) after PZT deposition. The difference in the effective mobility and the shift in the threshold voltage in the $I-V$ curves indicate that strain is formed after deposition of the LNO/PZT stack. Furthermore, as mentioned before, oxide deformation after PZT deposition can possibly change the interface trap density. At low field, Coulomb scattering is important and that is affected by the traps. Therefore, oxide deformation can also help to increase the low field mobility.

At high fields on the other hand surface roughness scattering is important which could also be affected by strain. As discussed in section 5.4.1, we expect biaxial tensile stress in the PZT due to difference in CTE when no external bias is applied. As shown in Fig. 5.6, the step coverage of the PZT is not as ideal as depicted in Fig. 5.1. Therefore, we expect that the stress profile is not determined by the PZT layer on the BOX layer, but more by the PZT layer located at the fin sidewall. Therefore, for narrow fins $\left(W_{\mathrm{FIN}}<<H_{\mathrm{FIN}}\right)$, a strain gradient parallel to the surface of the $(1 \overline{1} 0)$ fin sidewall is important. In this case, for narrow fins, biaxial compressive stress along the fin height and channel length direction is formed. This results in uniaxial tensile stress along the fin width direction and consequently increases high field electron mobility [5].

For narrow fins under both tensile and compressive stress along the fin height direction, the conduction band valleys move down in energy. The latter would give a larger impact both in the band offset and increased mobility [5]. This will result in a negative threshold 
voltage shift after deposition [2]. This can cause a relatively small part of the shift in threshold voltage observed in Fig. 5.10 (a).

Compressive stress along the fin height direction move the $\Delta_{2}$ ("light") electron valleys down in energy. The conductivity effective mass of electrons reduces, thereby increasing the electron mobility. Since we expect compressive stress along the channel length, this stress configuration is present for narrow fins in our devices (for $W_{\mathrm{FIN}}=30$ and $100 \mathrm{~nm}$ ). We can observe it in Fig. 5.19 (a) at low field (moderate inversion) region.

For wide fins ( $\left.W_{\mathrm{FIN}}>>H_{\mathrm{FIN}}\right)$ on the other hand, the sidewalls can be neglected compared to the top surface of the fin. Therefore, wide fins are expected to behave like a planar (001) surface (as in a (001) bulk MOSFET). Since the Si substrate is much thicker than the Si fin height, the intermediate layers and the PZT layer, the in-plane biaxial tensile stress formed in the PZT layer will be transferred to the underlying layers down to the Si substrate [22]. Hence, biaxial tensile stress along the fin width and channel length direction is formed. This configuration would result in uniaxial compressive stress along the fin height direction.

Compressive stress along the fin height direction move $\Delta_{2}$ ("light") electron valleys down in energy. The conductivity effective mass of electrons decreases and thereby electron mobility increases. This stress configuration is present for wide fins in our devices (for $W_{\text {FIN }}=1000 \mathrm{~nm}$ ) which is (001) surface oriented. This can be seen in Fig. 5.19 and 5.20 (a)(b).

In summary, before any $V_{\pi}$ biasing, the low field mobility (moderate inversion) increases possibly by the oxide deformation that reduces the effective interface traps at the $\mathrm{Si} / \mathrm{SiO}_{2}$ interface. On the other hand, the CTE difference formed strain in Si influences the high field mobility (strong inversion). For wide fins, the high field mobility increases due to biaxial tensile stress along the fin width and channel length direction in the (001) surface. For narrow fins, the mobility also increases due to biaxial compressive stress along the fin height and channel length direction in the $(1 \overline{1} 0)$ surface of the fin sidewall.

\section{Biasing the PZT layer:}

A bias varying from $-3 \mathrm{~V}$ to $3 \mathrm{~V}$ has been applied over the PZT layer and the mobility has been calculated. According to Fig. 5.1 (a), we expect upward deflection under negative whereas downward deflection under a positive applied field [23]. Therefore, for a negative field, we expect the narrow $\mathrm{Si}$ fin to be more compressively stressed along the channel length direction and tensile stress in fin height direction and the mobility decreases. On the other hand, for a positive field, more tensile stress is formed along the channel length direction and compressive stress in fin height direction and the mobility increases [5]. For a wide fin the situation is different. When applying a positive applied 
field we have biaxial tensile stress at the (001) surface which tends to increase the electron mobility [4]. On the other hand, a negative bias will yield almost no change in the mobility.

At low field: Fig. 5.19 (a) shows an increasing trend of the effective mobility of up to $20 \%$ at a positive bias for $30 \mathrm{~nm}$ and $100 \mathrm{~nm}$ wide fins. The trend is the opposite at a negative applied bias. For the $1000 \mathrm{~nm}$ wide device however, there is almost no change. Fig. 5.19 (b) shows that there is almost no change in mobility for single fin devices. Therefore, the trend of mobility for 5 -fin $30 \mathrm{~nm}$ and $100 \mathrm{~nm}$ wide devices can be explained by data from literature.

At high field: In Fig. 5.20 (a) a smaller decrease in effective mobility at a negative bias is observed. For a positive bias, it is almost constant in contrast to Fig. 5.19 (a). For single fin devices presented in Fig. 5.20 (b), the mobility is almost the same over the whole bias range. Therefore, the trend of mobility for 5 -fin $30 \mathrm{~nm}$ and $100 \mathrm{~nm}$ wide devices at negative PZT bias can be explained by the literature data.

The mobility dependency on the amount of fins could be related to the strain formed by the PZT layer along the surface between parallel fins. Each fin could form a kind of side clamping surface for the PZT layer in between parallel fins. The strain would then be more profound at the sidewalls of the middle 3-fins. Since PZT is biaxial tensile stressed after deposition, we expect a compressive stress along the fin width direction. This effect would be more profound in the narrow fin devices. In Fig. 5.18 (f), for a single fin device, the mobility is decreased more after deposition than in Fig. 5.17 (e), for a 5-fin device. This difference could be due an increased compressive stress along the fin width for a 5fin device due to the clamping effect; which would result in turn in more tensile stress along the channel length direction.

In summary, a threshold voltage shift is observed after the piezoelectric layer deposition due to stress in the oxide and Si layers. A further shift is obtained under an applied bias over the piezoelectric layer, which is attributed to the leakage current induced surface potential change. The SS is also modified after deposition and under PZT bias. Change in the trap density due to oxide deformation could be a reason for this effect. Furthermore, a change in the electron mobility is observed after the PZT deposition. The low field mobility change could again be related to a change in effective trap density due to the stressed oxide layer. This change in mobility shows a fin width dependency also. Regarding the obtained device characteristics, PiezoFETs show a change in SS and mobility as a result of the presence of the piezoelectric layer. 


\subsubsection{C-V characterization}

The capacitance-voltage $(C-V)$ characteristics of the PiezoFETs can be used to study any degradation of the gate oxide properties after PZT deposition. $C-V$ measurements were also done to measure the equivalent capacitance of the structure. Dedicated $C-V$ test structures together with their open and short de-embedding pairs were designed as described in chapter 4 . Each capacitance measurement was performed by de-embedding the contact pad as well as the parasitic connections. The capacitor dimensions are summarized in Table 5.2. The measurements were performed at $V_{a c}=20 \mathrm{mV}$ and at $1 \mathrm{MHz}$ frequency.

Fig. 5.21 shows the $C-V$ curves obtained from parallel fin gate capacitors for 60 fins with $W_{\mathrm{FIN}}=60 \mathrm{~nm}, L_{\text {gate }}=20 \mu \mathrm{m}, L_{\text {pigate }}=4 \mu \mathrm{m}$. The capacitance values were calculated using Eq. (4.15). Since $\varepsilon_{\text {PZT }}$ was determined to be 600 in chapter 3 , the calculated PZT capacitance is $9.2 \times 10^{-12} \mathrm{~F}$ and the calculated oxide capacitance is $1.36 \times 10^{-12} \mathrm{~F}$. Since the capacitances are in series, the equivalent capacitance equals $1.0 \times 10^{-12} \mathrm{~F}$. The calculated and measured capacitance values are presented in Table 5.4 showing good agreement. The relatively small difference between the calculated and measured equivalent capacitance could be due the voltage dependency of the dielectric constant of PZT which was not incorporated in the calculations.

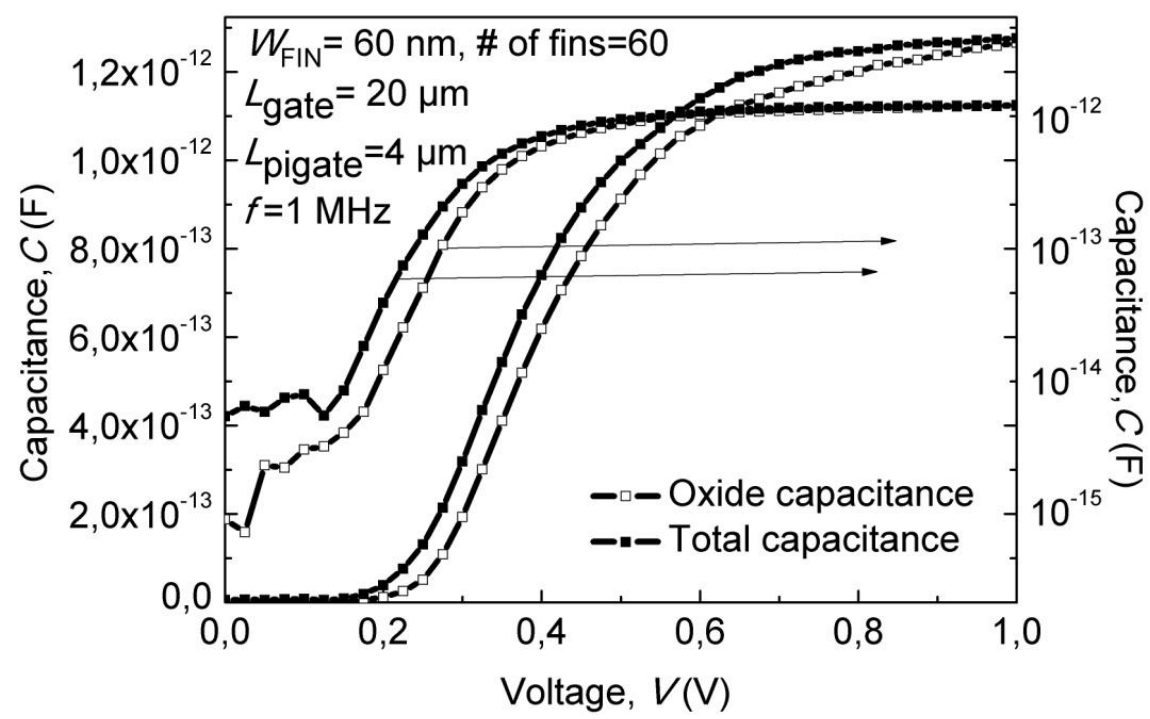

Fig. $5.21 \mathrm{C}-V$ characteristics of the gate capacitor test structure containing 60 fins with $W_{\mathrm{FIN}}=60$ $\mathrm{nm}, L_{\text {gate }}=20 \mu \mathrm{m}, L_{\text {pigate }}=4 \mu \mathrm{m}$. Measurements were performed at $f=1 \mathrm{MHz}$ with $V_{a c}=20 \mathrm{mV}$. 
Table 5.4 Calculated and measured capacitance values for a parallel fin C-V test structure

\begin{tabular}{ccc}
\hline $\begin{array}{c}W_{\text {FIN }}=60 \mathrm{~nm}, L_{\text {gate }}=20 \mu \mathrm{m} \\
L_{\text {pigate }}=4 \mu \mathrm{m}\end{array}$ & Calculated & Measured \\
\hline \hline $\begin{array}{l}\text { Oxide capacitance of } \\
\text { PiezoFET with } 60 \text { fins }\end{array}$ & $1.36 \times 10^{-12} \mathrm{~F}$ & $1.28 \times 10^{-12} \mathrm{~F}$ \\
$\quad \begin{array}{l}\text { Total capacitance of } \\
\text { PiezoFET with } 60 \text { fins }\end{array}$ & $1.00 \times 10^{-12} \mathrm{~F}$ & $1.25 \times 10^{-12} \mathrm{~F}$ \\
\hline \hline
\end{tabular}

This measurement is an indication that there is hardly any degradation in the properties of the oxide after PZT deposition. Furthermore, the $C-V$ curve is also plotted in semi-log scale to investigate the trap related slope degradation. No serious change in the slope is observed, meaning that Si-oxide interface is not degraded due to PZT deposition. No further measurement data is presented with different area capacitors since the equivalent capacitance is close to that of the oxide capacitance.

The $C-V$ measurement of the PZT layer on the channel was performed with dedicated test structures presented in Fig. 5.3. However, the relatively high leakage current (see e.g. Fig. 5.14) disturbed the measurements.

Bias temperature stress (BTS) measurements were also done to determine the amount of mobile charge concentration of the PiezoFET. The $C-V$ test structures were used for these measurements. The experimental methodology used was as follows. During the measurement, the device was heated up to $150-200{ }^{\circ} \mathrm{C}$ and a gate bias was applied to produce an electric field in the order of $\mathrm{MV} / \mathrm{cm}$ for 5-10 min causing mobile charge drifting to one interface. Then, the device was cooled down to room temperature under the applied field and a second $C-V$ measurement is performed. The same procedure was repeated for the opposite field. The mobile charge can then be determined from the flatband voltage shift of the $C$-V curve [24].

Fig. 5.22 shows the $C-V$ curves of the FinFET and PiezoFET before and after the BTS measurement. Before the BTS measurement, the capacitors were heated up to $200{ }^{\circ} \mathrm{C}$ and biased for $10 \mathrm{~min}$ under $\pm 3 \mathrm{~V}\left(E \approx \pm 2 \times 10^{5} \mathrm{~V} / \mathrm{cm}\right)$. Fig. 5.22 (a) shows the $C-V$ measurements before PZT deposition. Figs. 5.22 (b) and (c) show the $C-V$ measurements from bottom gate to channel and from top gate to channel, respectively. 

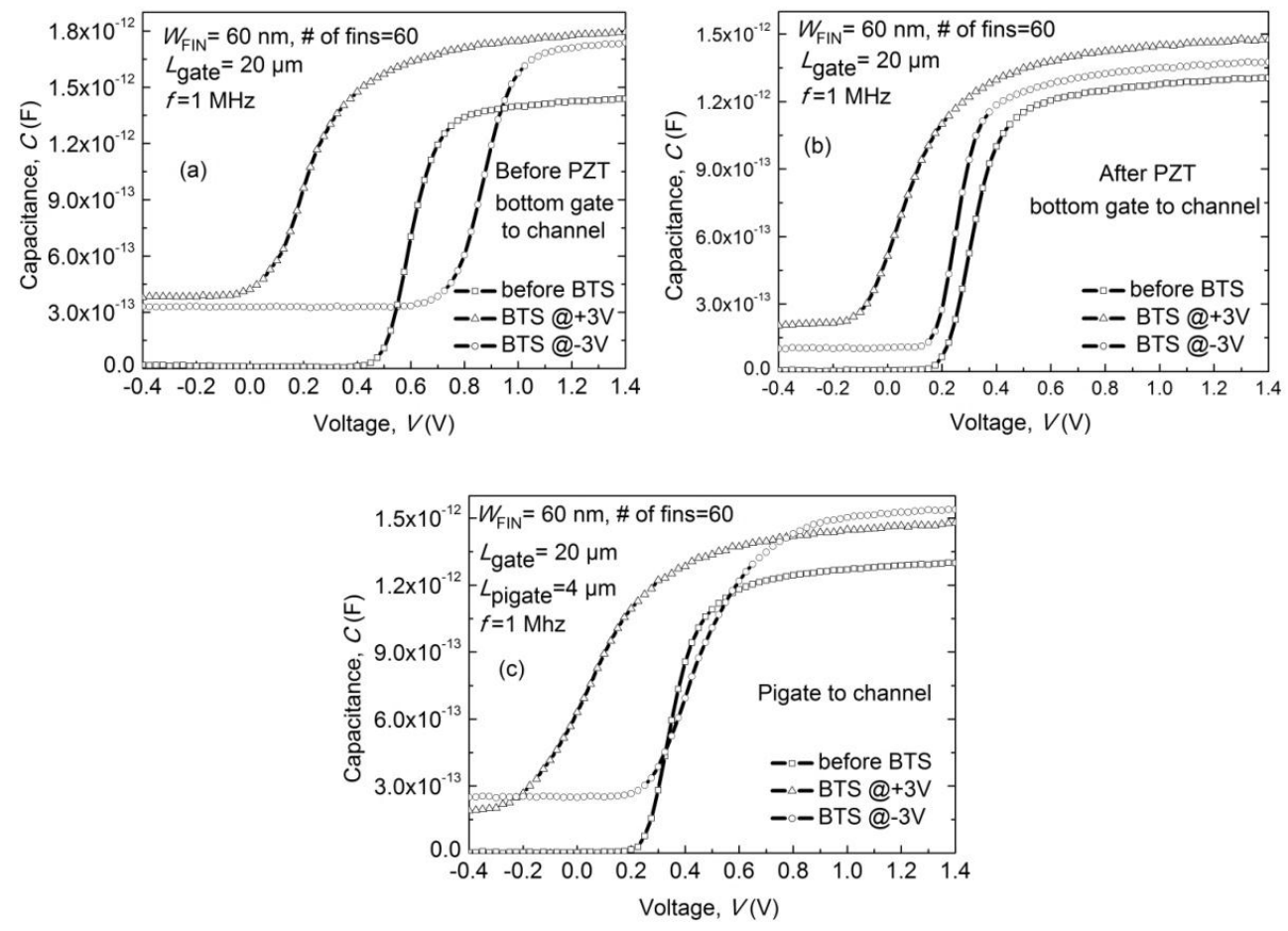

Fig. $5.22 \mathrm{C}-V$ characteristics of the capacitor test structure with 60 fins and $W_{\mathrm{FIN}}=60 \mathrm{~nm}, L_{\text {gate }}=20$ $\mu \mathrm{m}, L_{\text {pigate }}=4 \mu \mathrm{m}$ before and after the BTS (at $200^{\circ} \mathrm{C}$ for $10 \mathrm{~min}$ ) (a) before PZT deposition, (b) after PZT deposition, bottom gate to channel capacitance, (c) after PZT deposition, pigate to channel capacitance. Measurements were performed at $f=1 \mathrm{MHz}$ with $V_{a c}=20 \mathrm{mV}$.

The general trend is that a relatively small negative shift has been observed in the threshold voltage after the capacitor has been stressed. The relatively small shift in threshold voltage is positive after stressing the capacitor at $200{ }^{\circ} \mathrm{C}$ for $10 \mathrm{~min}$ under $-3 \mathrm{~V}$ bias. This shift has been observed before the PZT deposition also. This means that the gate oxide $\left(\mathrm{SiO}_{2}\right)$ layer contained already some mobile charge before PZT deposition and there is no sign of additional mobile charge due to the PZT layer. Since the threshold voltage shift is much smaller than the actual shift observed when biasing the PiezoFET, the mobile ions do not play a significant role in this effect. These mobile ions were possibly formed during the process of the FinFETs, hence before the PZT deposition. The results emphasize that $\mathrm{PZT}$ is compatible with FinFETs.

\subsection{Conclusions}

This chapter presents the processing and characterization of a novel four terminal device structure called the PiezoFET. The processing started by removing Al from the contact pads of the FinFETs. Afterwards, the PZT/LNO stack was deposited by PLD. Patterning of these layers was performed by wet etching and finally the Pt/Ti contact pads were 
defined by a lift off process. For comparison, AIN deposited device characteristics have been presented as well.

Optical, physical, and ferroelectric characterization of the PZT layer was performed by XRD, LDV and P-E measurements. The piezoelectric activity of the PZT layer on the FinFETs was evidenced by its high $d_{33}(\approx 100 \mathrm{pm} / \mathrm{V})$ response, especially from the channel region.

The effect of the piezoelectric layer on the transport properties in the Si channel was studied by $I-V$ measurements. No harmful effects were observed in device properties after piezoelectric layer deposition. A negative shift is observed in the $I_{\mathrm{d}}-V_{\mathrm{gs}}$ curves after PZT and AIN deposition. This effect is related to the coefficient of thermal expansion (CTE) difference formed strain in the $\mathrm{Si}$ and the oxide. It causes a strong gate oxide deformation possibly affecting the band alignment and related charge trapping. The latter was supported by the observed change in the SS.

When the piezoelectric layer was biased at a constant field, a relatively high positive and negative $\Delta V_{\text {th }}$ was observed in the FinFET devices under high piezoelectric leakage current. An analogue electrical circuit is presented to explain this effect. The leakage paths are most probably created due to poor step coverage of the PZT and AIN layers around the fins. The extracted interface trap density shows a dependence on the PZT bias. By applying a bias over the PZT layer deforms the oxide layer and Si further which appears to change the amount of effective interface traps and hence SS.

The transport properties of the transistors were studied in terms of mobility. It is shown that the mobility at strong inversion decreases for $30 \mathrm{~nm}$ wide fin devices (stronger for a single fin device) whereas it increased (of up to $\approx 50 \%$ ) for $100 \mathrm{~nm}$ and $1000 \mathrm{~nm}$ wide fin devices after PZT deposition. An increase in the mobility after PZT deposition is again related to the strain formed by CTE difference. The same effect is also observed for AIN deposited devices. The type of strain in the narrow fins and wide fin devices are different. For a narrow fin, a compressive stress is formed along the channel length direction at the (11̄0) surface of the fin sidewall, resulting in a lower mobility. For a wide fin device the stress is tensile at the (001) surface and the mobility increases accordingly.

Applying a bias over the PZT layer has also an effect on the mobility (up to $\approx 20 \%$ ), showing the converse piezoelectric effect. In the moderate inversion regime (near threshold voltage), the mobility decreases when the PZT layer is negatively biased. When the applied bias is positive, the $30 \mathrm{~nm}$ and $100 \mathrm{~nm}$ wide 5 -fin devices show a higher mobility. This is related to an increased tensile stress along the channel length direction. A decrease of the mobility under negative bias, on the other hand, is related to an increased compressive stress along the channel length direction. Single fin devices show 
hardly any effect in mobility. The strain effect is less in single fin devices possibly due to the absence of the fin sidewall clamping compared to multi-fin devices.

We obtain a less significant decrease in the effective mobility at the strong inversion mode of the 5-fin device when a negative bias is applied. However, when a positive bias is applied, the mobility is not affected. Furthermore, the mobility of a single fin device is almost constant over the whole bias range. The weak dependence of the mobility on the applied bias in strong inversion region is related to a quantization of carriers, which counteracts the stress induced repopulation of the electron valleys.

Furthermore, $C-V$ measurements were performed to analyze any degradation in the oxide properties after PZT deposition. Almost no change has been observed in the $C-V$ curves of the FinFETs after PZT deposition. BTS measurements were also performed to observe any effect due to mobile ions. No change is observed in the curves before and after PZT deposition. Therefore, there is no additional mobile charge related effects in the PiezoFET due to PZT layer.

With all these results, we conclude that PZT is successfully integrated with FinFETs and no harmful effects have been observed in the underlying device structure. In addition, despite the issues of the leakage current and poor step coverage, the measurements from different device configurations indicate a change in underlying device properties when the PZT layer is actuated.

\section{References}

[1] N. Setter, D. Damjanovic, L. Eng, G. Fox, S. Gevorgian, S. Hong, A. Kingon, H. Kohlstedt, N. Y. Park, G. B. Stephenson, I. Stolitchnov, A. K. Taganstev, D. V. Taylor, T. Yamada, and S. Streiffer, "Ferroelectric thin films: Review of materials, properties, and applications", Journal of Applied Physics, 100, pp. 051606-051606-46, 2006

[2] T. van Hemert and R.J.E Hueting, "Piezoelectric Strain Modulation in FETs", IEEE Trans. Electron Devices, 60, pp. 3265-3270, 2013.

[3] S. Trolier-McKinstry, "Draft 16 of a working document for a proposed standard to be entitled: IEEE standard definitions of terms associated with ferroelectric and related materials", IEEE Transactions on Ultrasonics, Ferroelectrics and Frequency Control, 50, pp. 1613-1646, 2003.

[4] T. Skotnicki, C. F.-Beranger, C. Gallon, F. Boeuf, S. Monfray, F. Payet, A. Pouydebasque, M. Szczap, A. Farcy, F. Arnaud, S. Clerc, M. Sellier, A. Cathignol, J.-P. Schoellkopf, E. Perea, R. Ferrant, and H. Mingam "Innovative Materials, Devices, and CMOS Technologies for Low-Power Mobile Multimedia", IEEE Transactions on Electron Devices, 55, pp. 96-129, 2008.

[5] N. Serra and D. Esseni, "Mobility Enhancement in Strained $n$-FinFETs: Basic Insight and Stress Engineering", IEEE Transactions on Electron Devices, 57, pp. 482-490, 2010.

[6] B. A. Auld, "Acoustic fields and waves in solids" vol. 1, John Wiley and Sons, Inc., 1973.

[7] B. Jaffe and W.R. Cook, "Piezoelectric Ceramics", Academic Press, London, 1971.

[8] G. Han, J. Ryu, W. H. Yoon, J J. Choi, B.-D. Hahn, J.W. Kim, D. S.Park, C. W. Ahn, S. Priya and D. Y. Jeong, "Stress-controlled $\mathrm{Pb}\left(\mathrm{Zr}_{0.52} \mathrm{Ti}_{0.48}\right) \mathrm{O}_{3}$ thick films by thermal expansion mismatch between substrate and $\mathrm{Pb}\left(\mathrm{Zr}_{0.52} \mathrm{Ti}_{0.48}\right) \mathrm{O}_{3}$ film", Journal of Applied Physics, 110, pp. 124101, 2011. 
[9] K. G. Lyon and G. L. Salinger and C. A. Swenson and G. K. White. "Linear thermal expansion measurements on silicon from 6 to 340 K", Journal of Applied Physics, 48, 865-868, 1977.

[10] K. Kurihara, M.Kondo, K. Sato, M. Ishii, N. Wakiya and K. Shinozak, "Electrooptic Properties of Epitaxial Lead Zirconate Titanate Films on Silicon Substrate", Japanese Journal of Applied Physics, 46, pp. 6929-6932, 2007.

[11] W. H. Xu, D. Lu, and T.-Y. Zhang, "Determination of residual stresses in $\mathrm{Pb}\left(\mathrm{Zr}_{0.53} \mathrm{Ti}_{0.47}\right) \mathrm{O}_{3}$ thin films with Raman spectroscopy", Appl. Phys. Lett. 79, pp. 4112-4114, 2001.

[12] M. H. Zhao, R. Fu, D. Lu, T.-Y. Zhang, “Critical thickness for cracking of $\mathrm{Pb}\left(\mathrm{Zr}_{0.53} \mathrm{Ti}_{0.47}\right) \mathrm{O}_{3}$ thin films deposited on Pt/Ti/Si(100) substrates", Acta Materiala, 50, pp .4241-4254, 2002.

[13] A. Rusu, G. A. Salvatore, D. Jimenez, and A. M. Ionescu, "Metal-Ferroelectric-Meta-Oxidesemiconductor field effect transistor with sub-60mV/decade subthreshold swing and internal voltage amplification", IEEE International Electron Devices Meeting (IEDM) Technical Digest, 2010.

[14] S. L. Miller and P. J. McWhorter, "Physics of the ferroelectric nonvolatile memory field effect transistor", J. Appl. Phys., 72, pp. 5999, 1992.

[15] T. B. Hook and T. -P. Ma, "Electron trapping during high-field tunneling injection in metal-oxidesilicon capacitors: The effect of gate-induced strain", J. Appl. Phys., 62, pp. 931-938, 1987.

[16] T. Irisawa, T. Numata, N. Hirashita, T. Tezuka, N. Sugiyama, S. Takagi, "Physical understanding of strain-induced modulation of gate oxide reliability in MOSFETs", IEEE Trans. Electron Devices, 55, pp. 3159-3166, 2008.

[17] S. Takagi, A. Toriumi, M. Iwase, and H. Tango, "On the universality of inversion layer mobility in Si MOSFET's: Part II-effects of surface orientation", IEEE Transactions on Electron Devices, 41, pp. 2363-2368, 1994.

[18] T. Rudenko, V. Kilchytska, N. Collaert, M. Jurczak, A. Nazarov, and D. Flandre, "Carrier Mobility in Undoped Triple-Gate FinFET Structures and Limitations of Its Description in Terms of Top and Sidewall Channel Mobilities", IEEE Transactions on Electron Devices, 55, pp. 3532-3541, 2008.

[19] C. T. Sah and T. H. Ning and L. L. Tschopp, "The scattering of electrons by surface oxide charges and by lattice vibrations at the silicon-silicon dioxide interface", Surface Science, 32, pp. 561-575, 1972.

[20] F. Stern, "Quantum properties of surface space charge layers", CRC Critical Reviews in Solid State Sciences, 4, pp. 499-514, 1974.

[21] Y. C Cheng, and E. A. Sullivan, "On the role of scattering by surface roughness in silicon inversion layers", Surface Science, 34, pp. 717-731, 1973.

[22] M. H. Zhao, R. Fu, D. Lu, and T.-Y. Zhang, "Critical thickness for cracking of $\mathrm{Pb}\left(\mathrm{Zr}_{0.53} \mathrm{Ti}_{0.47}\right) \mathrm{O}_{3}$ thin films deposited on Pt/Ti/Si(100) substrates", Acta Materialia, 50, pp. 4241-4254, 2002.

[23] N. Jalili, J. Wagner and M. Dadfarnia, "A piezoelectric driven ratchet actuator mechanism with application to automotive engine valves", Mechatronics, 13, pp. 933-956, 2003.

[24] D. K. Schroder, "Semiconductor Material and Device Characterization", 3 rd ed., Wiley interscience, 2006. 


\section{Conclusions and recommendations}




\subsection{Conclusions}

In this work, we investigated strained SOI FinFETs and the feasibility of usage and implementation of the converse piezoelectric effect as a stressor. Strain is often applied in semiconductor research and technology and in research projects to improve the device performance. We have studied the strain in FinFETs formed by the difference in coefficient of thermal expansion (CTE) of the thin film materials used. In addition, a next possible generation strained device structure, the PiezoFET, was realized and characterized. In this case, PZT was chosen as a stressor to obtain tunable strain due to its high piezoelectric constant.

$I-V$ measurements were used to analyze the strain effects in the bandgap of $(1 \overline{1} 0) /[110]$ oriented n-type strained FinFETs obtained from NXP. Raman spectroscopy was also used to directly characterize the device level strain. An offset in conduction band energy down to $\approx-40 \mathrm{meV}$ was determined from the electrical measurements. Depending on the dimensions of the fins, the effect of strain or strain including quantum confinement effects is present in the devices. Strain was formed due to a difference in CTE of TiN and $\mathrm{Si}$. In addition, Raman spectroscopy measurements indicated a maximum of $-0.88 \%$ strain in the Si channel. Good agreement was obtained in strain values determined by Raman spectroscopy, FEM simulations and holographic interferometry. Therefore, Raman spectroscopy was shown to be a good method to obtain strain information from FinFET structures. Furthermore, the result obtained from the electrical analysis could be explained by using the strain-fin width relation obtained by these techniques.

Sub-100 nm thick thin-film PZT/LNO stacks on an encapsulated TiN electrode were studied to characterize their properties. A promising effective piezoelectric coefficient $d_{33, f}$ was obtained with a maximum of $53 \mathrm{pm} / \mathrm{V}$ for 75 and $100 \mathrm{~nm}$ thick films. Extensive analysis was done by capacitance-voltage $(C-V)$ and current-voltage $(I-V)$ measurements. The results indicate the presence of a passive layer at the electrode interface. A relative dielectric constant of the PZT layer of 600 was determined. The effective thickness and the relative dielectric constant of the passive layer was estimated to be $2.1 \mathrm{~nm}$ and 23, respectively. A lower than expected barrier height of $0.32 \mathrm{eV}$ was obtained which is possibly caused by ferroelectric polarization induced barrier lowering. It was also shown that the leakage current can be described with a diffusion-based rather than a pure Schottky emission model.

In this study, FinFETs were designed, fabricated and characterized in MESA+. They serve as a base for our work on the PiezoFETs. The fabricated devices were analyzed by resistivity, $I-V$ and $C-V$ measurements. Devices with subthreshold swing (SS) of 76 $\mathrm{mV} / \mathrm{dec}$, a low leakage and high $I_{\text {on }} / I_{\text {off }}\left(\approx 10^{8}\right)$ current were obtained. Good performance of our $(1 \overline{1} 0) /[110]$ oriented FinFETs was evidenced by these figures and by 
comparison with the NXP-FinFETs characteristics with the same orientation. In addition, good effective mobility-electric field curve for long gate lengths $(\approx 10 \mu \mathrm{m})$ were obtained in agreement with data from the literature.

Using these FinFETs as a base, PiezoFET devices were processed and characterized. No degradation was observed in the device characteristics after the piezoelectric layer deposition on the FinFETs. It is an indication of compatibility of PZT layer with Si device technology. The PZT capacitor showed a high piezoelectric response on the FinFETs, $d_{33} \approx$ $100 \mathrm{pm} / \mathrm{V}$, especially from the channel region. $I_{\mathrm{d}}-V_{\mathrm{gs}}$ measurements indicated a threshold voltage shift after PZT or AIN deposition without any bias. This shift appears to be related to the strain formed in the $\mathrm{Si}$ and in the oxide due to the difference in the CTE of Si and the covering layers. This strain deforms the relatively low stiff gate oxide and it can possibly affect the band alignment resulting in a change in effective interface trap density. The observed change in SS was a verification for the difference in trap charge density.

A relatively high positive and negative $\Delta V_{\text {th }}$ was observed under constant bias of the PZT piezoelectric layer. Furthermore, a high piezoelectric leakage current was observed in the same measurements due to poor step coverage of the piezoelectric layer. It was found that the leakage current through the piezoelectric layer and a comparable gate stack resistance resulted in a voltage drop over the bottom gate. As a result, the surface potential is increased by the voltage drop over the gate stack altering the $V_{\text {th }}$. Since single fin devices with a large fin width showed less leakage, we conclude that the leakage paths are mainly located at the fin sidewalls. This emphasizes the importance of the step coverage of the piezoelectric layer. Applying a bias over the PZT layer changed the SS. This could be due to further deformation of the gate oxide layer and $\mathrm{Si}$, which results in a change of the effective interface trap density and hence SS.

The strong inversion mobility decreased for $30 \mathrm{~nm}$ wide fin devices whereas it increased (up to $\approx 60 \%$ ) for $100 \mathrm{~nm}$ and $1000 \mathrm{~nm}$ wide fin devices after PZT deposition. Strain in the channel and gate oxide formed by the difference in CTE could be a reason of an increase in the mobility. The same effect was also observed for devices with an AIN layer. The strain affects narrow fins and wide fin devices differently. For a narrow fin, the fin sidewall is more important and after deposition compressive stress is formed along the $(1 \overline{1} 0)$ fin sidewall, resulting in a lower mobility. For a wide fin, the (001) device surface is more important. In this case stress is tensile at the (001) surface and the mobility increases accordingly.

Biasing the PZT layer changed the mobility up to $\approx 20 \%$. This is an indication of the converse piezoelectric effect. When the PZT layer was negatively biased, we observed a decrease in mobility in the moderate inversion regime. When the applied bias was 
positive, a higher mobility was observed for $30 \mathrm{~nm}$ and $100 \mathrm{~nm}$ wide 5 -fin devices. This indicates an increase in tensile stress along the channel length direction. When the applied bias was negative, mobility decreased. This could be an indication of an increase in compressive stress along the channel length direction.

Furthermore, the $C-V$ curves of the gate capacitor before and after deposition of the piezoelectric layer were almost the same. Bias temperature stress measurements were also performed and again no change was observed in the curves before and after PZT deposition. Therefore, we conclude that there is no additional mobile charge related effects in the PiezoFET induced by the PZT layer.

The fabricated PiezoFET is a good candidate for several applications. There is a possibility to obtain a lower SS than the base transistor using a piezoelectric layer. The SS of a device can also be controlled up to certain limits. Therefore, it can be used as an alternative FET design. Furthermore, the higher effective mobility compared to its base FinFETs makes it a candidate as a replacement of the FinFET. In addition, due to the large shifts in $V_{\text {th }}$ values, it can be applied as a memory with a large memory window of about $3 \mathrm{~V}$. Such a memory device based on the leakage current has been patented before [1]. Since this memory device does rely on a ferroelectric hysteresis effect, the memory window is more stable than FeRAMs.

\subsection{Recommendations}

The converse piezoelectric effect formed strain concept is a good candidate to obtain steep sub-threshold devices. Our results have demonstrated the feasibility and great potential of the concept. However, considering our results, there are several points that can be improved.

Since the converse piezoelectric effect relies on the mechanical displacement, it is important to keep the interlayer thicknesses as small as possible. The thickness of LNO as a PZT buffer layer cannot be decreased further since, as shown in chapter 3, the growth properties of PZT show high dependence on this layer thickness. However, the thickness of the underlying layers can be decreased for this purpose. Replacing the $\mathrm{SiO}_{2}$ layer of 11 $\mathrm{nm}$ thickness by a high-k $\mathrm{HfO}_{2} / \mathrm{SiO}_{2}$ layer of 2-3 nm thickness could be one way. In addition, the thickness of $12 \mathrm{~nm} \alpha$-Si encapsulating layer can be decreased down to $\approx 5$ $\mathrm{nm}$; below which the film is not continuous. In this way the stress in the channel can be improved.

The step coverage of the piezoelectric layer is very important. The leakage paths are easily formed when the layer is thinner on the sidewalls. This leakage current forms a voltage drop on the bottom gate. A better step coverage will result in an improved 
structure for electrical characterization and analysis of the PiezoFET concept. CVD or ALD techniques could be used to obtain good step coverage. Although ALD is not obvious for the PZT deposition, it can be used for AIN deposition. Preventing leakage will also help to understand the strain modulation effect on the device transport properties.

Since the FinFET/PiezoFET is a 3D device structure, strain components from the top and the sidewall have to be taken into account. In addition, the fin height needs to be optimized in the range of thickness of the piezoelectric layer to control the strain in the Si channels. Therefore, although higher strain values can be obtained using 3D narrow channels, there are some complications in understanding and calculating the individual components. Piezoelectric layers on planar surfaces, on the other hand, form a more uniform strain distribution in the Si channel. For this reason, using planar structures can be a good alternative for an extensive study on the PiezoFET device concept.

\section{Reference}

[1] I. K. Yoo, "Ferroelectric Memory Using Leakage Current and Multi Numeration System Ferroelectric Memory", United States Patent, Patent No 5,812,442, 1998. 


\section{Summary}

Strain is often applied in semiconductor technology to improve the device performance in a field effect transistor (FET). However, it increases the off-state current as well. In this work, we investigated so-called silicon-on-insulator (SOI) fin-shaped field-effect transistors (FinFETs) and the effects of strain formed by the difference in coefficient of thermal expansion (CTE) of the used materials. Near ideal SOI FinFETs were realized in the clean room of MESA+ Institute for Nanotechnology. They served as a base for our new strained device structure: the PiezoFET. The PiezoFET is a novel device in which the channel strain can be controlled by a piezoelectric stressor to keep the off-current the same while increasing the on-current. In this device structure, PZT was used as a stressor and the effects of strain modulation due to the converse piezoelectric (piezo) effect in these devices were shown.

Chapter 1 presents the motivation, the project description and the objectives. Concepts of steep sub-threshold devices reported from literature are summarized and the effects of strain in Si channels are addressed. The reasoning behind the preferred device configuration, the SOI FinFET, and the use of TiN as a bottom gate are discussed. A new device concept, called the PiezoFET, is introduced. In this device the converse piezoelectric effect from PZT is used to induce strain modulation in the Si channels.

In chapter 2 electrical analysis and Raman spectroscopy were used to study device level strain in (110) oriented n-type Si strained FinFETs. In these devices strain was formed by the difference in CTE of the Si fin and the TiN gate material. An offset in conduction band energy down to $\approx-40 \mathrm{meV}$ was determined from the electrical measurements. This offset is changing depending on the fin dimensions. It can be explained by counteracting quantum confinement and strain effects. Raman spectroscopy measurements indicated a maximum of -0.88 \% strain in the Si channel. The strain values obtained from Raman measurements, FEM simulations and holographic interferometry are in good agreement. Raman spectroscopy was shown to be a good method to obtain actual strain values in 
FinFET structures with nanometer dimensions. In addition, electrical measurement results could be explained by the picture drawn by these techniques.

In chapter 3 a brief introduction to ferroelectrics films and applications is given. Next the properties of thin PZT films used in the PiezoFET were investigated. A promising effective piezoelectric coefficient $d_{33, \mathrm{f}}$ was obtained with a maximum of $53 \mathrm{pm} / \mathrm{V}$ for 75 and 100 $\mathrm{nm}$ thick films. Extensive analysis was done by capacitance-voltage $(C-V)$ and currentvoltage $(I-V)$ measurements. The results indicate the presence of a passive layer at the electrode interface. A relative dielectric constant of the PZT layer of 600 was determined. The effective thickness and the relative dielectric constant of the passive layer was estimated to be $2.1 \mathrm{~nm}$ and 23, respectively. A lower than expected barrier height of 0.32 $\mathrm{eV}$ was obtained which is possibly caused by ferroelectric polarization induced barrier lowering. A good fit of the measurement data with theory revealed that leakage current can be described by a diffusion-based rather than a pure Schottky emission model.

In chapter 4 our FinFETs are described. These devices were designed and fabricated in the clean room of the MESA+ Institute for Nanotechnology. They were analyzed by resistivity, $I-V$ and $C-V$ measurements. Results revealed a near-ideal behavior of the FinFETs with a sub-threshold swing (SS) of $75 \mathrm{mV} / \mathrm{dec}$, a low leakage and high $I_{\text {on }} / I_{\text {off }}\left(\approx 10^{8}\right)$ ratio. The performance of our FinFETs was comparable with the NXP-FinFETs characteristics described in chapter 2 . In addition, the effective mobility-electric field curves for long gate lengths $(\approx 10 \mu \mathrm{m})$ were in agreement with data from the literature. These FinFETs served as the base for our work on the PiezoFETs described in chapter 5.

In chapter 5 the processing and characterization of the PiezoFETs are presented. After the PZT deposition no harmful effects were observed in the underlying device characteristics. In the $I_{\mathrm{d}}-V_{\mathrm{gs}}$ measurements a shift in threshold voltage was found. This shift is partly due to the strain formed in the Si channel by the difference in the CTE of Si and the layers on top. This strain also deforms the gate oxide resulting in a change in the effective trap density at the Si-gate oxide interface. This resulted in the observed change in the SS in our PiezoFETs.

The PZT capacitors in the PiezoFET devices showed a high piezoelectric response, $d_{33} \approx 100$ $\mathrm{pm} / \mathrm{V}$, especially from the channel region. Biasing the PZT layer indicated the action of the converse piezoelectric effect. It changed both the SS and the electron mobility. The change in SS could be due to a further deformation of the gate oxide layer and the Si, resulting in a change of the effective interface trap density and hence the SS. Also a change in the mobility up to $\approx 20 \%$ was measured. For low $V_{\mathrm{gs}}$ this is can be explained again by the change of the effective interface trap density; since then Coulomb scattering is important. However, for high $V_{\mathrm{gs}}$, this is more related to stress in the Si. When the 
applied bias was positive, a higher mobility was observed for $30 \mathrm{~nm}$ and $100 \mathrm{~nm}$ wide 5fin devices. This indicated an increase in tensile stress along the channel length direction. On the other hand, when the PZT layer was negatively biased, a decrease in mobility was found due to an increase in compressive stress along the channel length direction.

The fabricated PiezoFET device is a good candidate for several future applications. Due to the possibility to obtain a lower and controllable SS compared to the base transistors, it can be an alternative FET design. The higher effective mobility makes it a reference point for improvement of the FinFET characteristics. 


\section{Samenvatting}

Mechanische vervorming (strain) wordt in de halfgeleider technologie vaak toegepast om de prestaties van een veld-effect transistor (FET) te verbeteren. Het kan echter ook de uit-stroom verhogen. In dit werk hebben we de zogenaamde silicium op isolator (SOI) vinvormige (fin) veld-effect transistoren (FinFETs) onderzocht en de effecten van strain in de silicium (Si) vin. Deze strain kan worden gevormd door het verschil in thermische uitzettingscoëfficiënt van de gebruikte materialen. Zeer goede SOI FinFETs zijn in de stofarme ruimte van het MESA+ Instituut voor Nanotechnologie gemaakt. Zij dienden als basis voor onze nieuwe vervormbare structuur: de PiëzoFET. De PiëzoFET is een structuur waarin door middel van een piëzo-elektrisch materiaal vervorming in de vin kan worden bewerkstelligd, zodanig dat de uit-stroom hetzelfde blijft terwijl de aan-stroom verhoogd wordt. In deze structuur werd lood zirconaat titanaat (PZT) gebruikt als piëzo-elektrisch materiaal. De effecten als gevolg van de piëzo-elektrische werking in deze FinFET structuren werden aangetoond.

Hoofdstuk 1 geeft de motivatie, de beschrijving van het project en de opzet. Voorbeelden van elektronische elementen met steile sub-drempelstroom (steep sub-threshold devices) uit de vakliteratuur zijn samengevat en de effecten van de mechanische vervorming op het Si worden behandeld. De keuze van de SOI FinFET structuur en de toepassing van titanium nitride (TiN) als electrode wordt toegelicht. Een nieuw ontwerp, de zogenaamde PiëzoFET, wordt geïntroduceerd. Hierin wordt het inverse piëzo-elektrische effect van PZT gebruikt om strain in het Si te bewerkstelligen.

In hoofdstuk 2 worden elektrische metingen en Raman spectroscopiemetingen gebruikt om de strain in (110)-georiënteerd n-type Si vinnen in FinFETs te bestuderen. In deze structuren werd de strain veroorzaakt door het verschil in thermische uitzettingscoëfficiënten van het $\mathrm{Si}$ en het TiN electrode materiaal. Uit de elektrische metingen werd een verschuiving van de energie van de geleidingsband van $\approx-40 \mathrm{meV}$ bepaald. Deze verschuiving was afhankelijk van de afmetingen van de vinvormige 
structuren. Dit kon worden verklaard uit de wisselwerking van kwantumopsluiting en de effecten van strain in het Si. De Raman spectroscopiemetingen toonden een maximale strain van de Si vin van $-0.88 \%$. De waarden uit de Raman spectroscopiemetingen, eindige-elementen (FEM) simulaties en holografische interferometrie kwamen goed overeen. Raman spectroscopie bleek een goede methode om de werkelijke strain in Si FinFETs te bepalen.

In hoofdstuk 3 wordt allereerst een korte inleiding gegeven over ferro-elektrische lagen en de toepassingen daarvan. Daarna werden de eigenschappen van dunne PZT lagen voor de PiëzoFET onderzocht. We vonden een veelbelovende maximale effectieve piëzoelektrische coëfficiënt van 53 pm/V voor 75 en 100 nm gedimensioneerde PZT lagen. Een uitgebreide analyse aan de hand van capaciteit-spanning $(C-V)$ en stroom-spanning $(I-V)$ metingen werd uitgevoerd. De resultaten wezen op de aanwezigheid van een passieve laag bij het grensvlak met een elektrode. De effectieve dikte van deze laag werd geschat op 2,1 nm met een diëlektrische constante van 23 . De verkregen barrièrehoogte van 0,32 eV was lager dan verwacht. Dit wordt mogelijk veroorzaakt door ferro-elektrische polarisatie. Een goede overeenkomst van de meetgegevens met de theorie voor de lekstroom wijst op een model gebaseerd op diffusie boven dat van zuivere Schottky emissie.

In hoofdstuk 4 worden onze FinFETs beschreven. Deze structuren werden ontworpen en ze werden gemaakt in de stofarme ruimte van het MESA+ Instituut voor Nanotechnologie. De analyse bestond uit weerstands-, (I-V)- en $(C-V)$ metingen. De resultaten lieten zeer goede eigenschappen van de FinFETs zien met een sub-drempel zwaai (sub-threshold swing) SS van $75 \mathrm{mV} / \mathrm{dec}$, een lage lekstroom en een grote verhouding van de aan- en uit-stroom $\left(I_{\text {on }} / I_{\text {off }}\right) \approx 10^{8}$. De prestaties van onze FinFETs was vergelijkbaar met de FinFETs van NXP zoals beschreven in hoofdstuk 2. Bovendien was er een goede overeenstemming tussen de gemeten verbanden van de effectieve mobiliteit en het aangelegde elektrische veld voor lange Si vinnen $(\approx 10 \mu \mathrm{m})$ en de gegevens uit de vakliteratuur. Deze FinFETs dienden als basis voor het werk aan PiëzoFETs zoals beschreven in hoofdstuk 5 .

In hoofdstuk 5 worden het maken en de karakterisering van de PiëzoFETs gegeven. Na het aanbrengen van de PZT laag werden er geen schadelijke gevolgen in de onderliggende Si vinstructuren waargenomen. In de $I_{\mathrm{d}}-V_{\mathrm{gs}}$ metingen werd een verschuiving van de drempelspanning gevonden. Deze verschuiving is gedeeltelijk toe te schrijven aan de strain in het Si door het verschil in thermische uitzettingscoëfficiënten van het Si en de lagen erboven. Dit vervormt ook het poortoxide $\left(\mathrm{SiO}_{2}\right)$ van de $\mathrm{FET}$, wat een verandering in de dichtheid van de effectieve elektrische defecten (traps) aan het grensvlak van het Si en het $\mathrm{SiO}_{2}$. Dit verklaart de waargenomen verandering in de PiëzoFET. 
De capaciteiten van PZT in de PiëzoFETs lieten een grote piëzo-elektrische respons van $\approx 100 \mathrm{pm} / \mathrm{V}$ zien, in het bijzonder in de gebieden met de Si vinnen. Hier liet een elektrische voorspanning op de PZT laag de werking van het inverse piëzo-elektrische effect zien. Het veranderde zowel de SS als de elektronen mobiliteit. De verandering in SS wordt mogelijk veroorzaakt door een verdere vervorming van het $\mathrm{Si}$ en het $\mathrm{SiO}_{2}$. Dit verandert de dichtheid van de effectieve traps aan het grensvlak en ook de SS. Er werd ook een verandering van de mobiliteit tot $\approx 20 \%$ gemeten. Voor een lage $V_{\mathrm{gs}}$ kan dit weer verklaard worden door een verandering in de dichtheid van effectieve traps, omdat dan Coulomb verstrooiïng belangrijk is. Echter, voor hogere $V_{\mathrm{gs}}$ is dit meer direct gekoppeld aan de strain in het $\mathrm{Si}$. Als de voorspanning posifief was, werd in structuren van 5 vinnen van 30 en $100 \mathrm{~nm}$ breed een hogere mobiliteit gevonden. Dit wijst op een toename van de mechanische trekspanning in de lengterichting van de vinnen. Anderzijds, voor een negatieve voorspanning werd er een lagere mobiliteit gevonden, dit als gevolg van een mechanische drukspanning in de lengterichting van de vinnen.

De onderzochte PiëzoFET is een goede kandidaat voor verscheidene toekomstige toepassingen. Door de verkregen lagere en instelbare SS, vergeleken met de oorspronkelijke transistor, kan deze structuur gebruikt worden als een alternatief ontwerp van een FET. De hogere mobiliteit vormt een goed uitgangspunt voor de verbetering van FinFETs. 


\section{List of publications}

\section{Journal Papers}

- B. Kaleli , R.J.E. Hueting, M.D. Nguyen, R.A.M. Wolters, "Integration of a piezoelectric layer on Si FinFETs for tunable strained device applications", submitted to IEEE Transactions on Electron devices.

- B. Kaleli, M.D. Nguyen, J. Schmitz, R.A.M. Wolters, R.J.E. Hueting., "Analysis of ferroelectric and electrical properties of sub-100 nm thick PZT/LNO stacks on an encapsulated TiN electrode" submitted to Microelectronic Engineering.

- B. Kaleli, T. van Hemert, R.J.E. Hueting, R.A.M. Wolters, "Strain characterization of FinFETs using Raman Spectroscopy" Thin Solid Films, 541, pp. 57-61, 2013.

- T. van Hemert, B. Kaleli, R.J.E. Hueting, D. Esseni, M.J.H. van Dal, J. Schmitz, "Strain and conduction band offset in narrow n-type FinFETs", IEEE Transactions on Electron Devices, 60, 3 (2013), pp.01005-1010.

- B. Kaleli, M. Kulakci, R. Turan, "Mechanisms of light emission from terbium ions $\left(\mathrm{Tb}^{3+}\right)$ embedded in a Si rich silicon oxide matrix" Optical Materials, 34, 11 (2012) pp. 19351939.

- E. Yilmaz, B. Kaleli, R.Turan, "A systematic study on MOS type radiation sensors", Nuclear Instruments and Methods in Physics Research B 254 (2007) pp. 287-292.

\section{Conference presentations}

- B. Kaleli, M.D. Nguyen, J. Schmitz, R.A.M. Wolters, R.J.E. Hueting, "Analysis of ferroelectric and electrical properties of sub-100 nm thick PZT/LNO stacks on an encapsulated TiN electrode", International Conference on Micro and Nano Engineering, 2013. 
- B. Kaleli, T. van Hemert, R.J.E. Hueting, R.A.M. Wolters, "Strain Characterization of FinFETs using Raman Spectroscopy", European Material Research Society (E-MRS) spring meeting, May 2012, Strasbourg.

- T. van Hemert, B. Kaleli, R.J.E Hueting, D. Esseni, M.J.H. van Dal, J. Schmitz, "Extracting the Conduction Band Offset in Strained FinFETs from Subthreshold-Current Measurement", Proceedings of the 41st European Solid-State Device Research Conference (ESSDERC 2011), 12-16 Sep 2011, Helsinki, Finland pp. 275-278.

- M. Kulakci, B. Kaleli, R. Turan, "Photoluminescence and electroluminescence from Tb doped silicon rich $\mathrm{SiO}_{2}$ prepared by magnetron sputtering", European Material Research Society (E-MRS) fall meeting, November 2010, Boston.

- B. Kaleli, M. Kulakci, R. Turan, "Effect of annealing conditions on Light emission from Terbium lons $\left(\mathrm{Tb}^{+3}\right)$ embedded in a matrix" Nanoscience and Nanotechnology 2009 meeting (NanoTR5), Turkey, June 8-12, 2009. 


\section{Acknowledgements}

In these last pages, I would like to express my gratitude to many people for their contribution to this work and for taking a role in my life during these years.

First of all, my promoter, Rob Wolters: Your broad range of knowledge and calm personality helped to cope with the desperate situations. I was always looking forward to our Wednesday meetings because I knew that the discussions would help me to step forward. I sincerely thank for your support and supervision during this hard period.

My supervisor, Ray Hueting: You were available whenever I needed, which actually helped me to be in time with this work. You have been a good listener and reacted/responded well to any kind of questions, suggestions or discussions. My sincere thanks for your guidance and valuable contributions towards this work.

I would like to thank Jurriaan Schmitz for giving me the opportunity to carry out my research as a PhD student at the University of Twente (UT). Jurriaan, thanks for your support and advices during my PhD.

I use this opportunity to thank Klaus Reimann, NXP Semiconductors, for the fruitful discussions we had. Your comments helped very much to improve this thesis.

My special thanks to Dirk Gravesteijn, NXP Semiconductors, for his critical and very valuable comments on this thesis.

I would like to thank Minh Nguyen for his kind help and our clarifying discussions. I really appreciate the time you spent to help me with this work.

I spend quite some time in the cleanroom during this study. I would like to thank to MESA+ cleanroom staff for the technical support with the systems and their friendship! My special thanks to Ton Jenneboer, especially for his warm welcome and help during the first months.

I had very nice moments at the UT, thanks to our nice former and current SC group members. Annemiek, you have a big heart with full of love. Thanks for your help over these years and our lovely talks about everything but not work $; \cdot$. Your cheerfulness always helped to feel better. Tom, thanks for your support, especially at the very beginning. Sander, thanks for your help with the software and measurement tools. My SC 
roommates; but more of my friends: Jiahui, Balaji, Vidhu, Hao, Giulia, Tom tom, Kazmi, thanks for our nice get-togethers and all enjoyable moments!! I would like to extend my thanks to my other colleagues and former group members: Alexey, Cora, Marcin, Alessandro, Jan-Laurents, Remke, Boni, Pietro, Joost, Arjen, Deepu, Jiwu, Victor, Erik and Rodolf. Thank you all for creating a cozy atmosphere which did not let me to feel alone.

I would like to thank to ICD group members for making our office more enjoyable place, especially on Friday afternoons (-). Many thanks for your tasty contributions in SC (\& ICD) dinners!

My dear paranymphs, Sumy Jose and Alfons Groenland: Thanks for being on my side; I am grateful for your support! Sumy, I feel quite lucky that I got a chance to know you better after moving to Carre. Thanks for your great friendship, our super fun Zumba classes and a big smile on your face $;$. Alfons, my heartfelt thanks for your warm welcome and extensive help, especially during my first months in Twente. I enjoyed our about everything chats so much! Thank you for being so open minded; which helped a lot to form a good friendship in a short period of time.

My dear Turkish friends at UT: İmran Avcı, Akın Avcl, Pınar Şantemiz Gökberk, Berk Gökberk, Burcu Gümüşçü Sefünç, Mustafa Akın Sefünç, you valued my time with your presence over these years. It was a big pleasure for me to meet you. Many many thanks for all the nice moments!

My dear Turkish friends (also coming from METU), at TuE: İlker Doğan, Nurcan Yanarcan Doğan, Murat Mesta and Döndü Şahin, thanks for our long lasting get-togethers whenever I came to Eindhoven. I feel very lucky that you also moved to NL, which in turn helped me to feel at home whenever we met $;$.

Canim anneciğim, babacığım ve kardeşim: En zor zamanlarda, sizlere ihtiyacım oldugunda hep Skype'ın öbür ucundaydınız. Bana uzaklardan büyük destek oldunuz, çok teşekkür ederim! Berkay'cığım, kapak dizaynı için sana ayrıca teşekkür ediyorum. İyi ki varsınız!

My darling, inspiration, joy, strength, husband and everything: Efe. You are the best thing that happened to me! You are my safe port and I know that whatever you say would come true. Your presence gave me everything I needed to finish the PhD. Sensiz olmaz gözbebeğim, seni çok seviyorum

Buket Kaleli

30-10-2013 


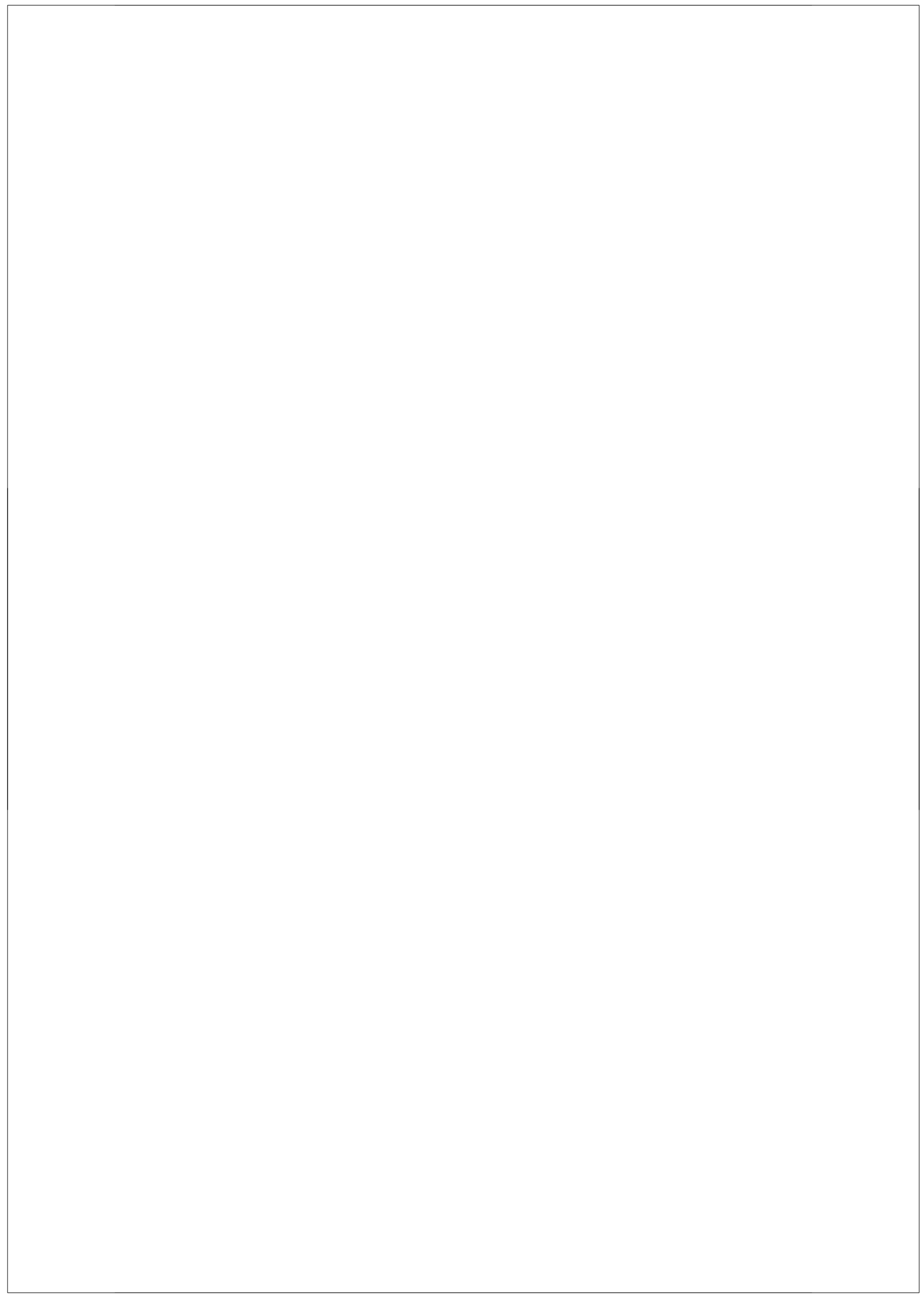

